\title{
Tissue-organization of Oscillating Cardiomyocytes via Matrix-mediated Biomechanical Signals
}

\author{
Dissertation \\ For THE AWARD OF THE DEGREE \\ "Doctor rerum naturalium" \\ of the University of Göttingen \\ within the doctoral program in physics \\ of the Georg-August University School of Science (GAUSS)
}

submitted by

Florian Spreckelsen

from Kassel

Göttingen, 2019 


\section{Thesis Committee}

Apl. Prof. Dr. Ulrich Parlitz,

Forschungsgruppe Biomedizinische Physik,

Max-Planck-Institut für Dynamik und Selbstorganisation

Prof. Dr. Stefan Klumpp,

Institut für Dynamik komplexer Systeme,

Georg-August-Universität Göttingen

Dr. Florian Rehfeldt,

Drittes Physikalisches Institut - Biophysik

Georg-August-Universität Göttingen

\section{Members of the Examination Board}

Referee: Prof. Dr. Stefan Klumpp,

Institut für Dynamik komplexer Systeme,

Georg-August-Universität Göttingen

$2^{\text {nd }}$ Referee: APL. Prof. Dr. Ulrich Parlitz, Forschungsgruppe Biomedizinische Physik,

Max-Planck-Institut für Dynamik und Selbstorganisation

Further members of the Examination Board:

Dr. Karen Alim,

Forschungsgruppe Biologische Physik und Morphogenese,

Max-Planck-Institut für Dynamik und Selbstorganisation

Dr. Claus Heussinger,

Institut für theoretische Physik,

Georg-August-Universität Göttingen

Dr. Florian Rehfeldt,

Drittes Physikalisches Institut - Biophysik,

Georg-August-Universität Göttingen

Prof. Dr. Peter Sollich,

Institut für theoretische Physik

Georg-August-Universität Göttingen

Date of oral examination: 2019-12-06 


\begin{abstract}
Tissue engineering, specifically engineered human myocardium (EHM), offers a promising therapeutic perspective for patients suffering from heart failure and also might improve drug development and testing. We therefore want to understand the interaction of heart muscle cells - cardiomyocytes $(\mathrm{CM})$ - in the very early stages of tissue generation. Deeper understanding may not only lead to improved fabrication but also will give insights into tissue organization in mammalian hearts in general.

Therefore a mathematical model of mechanically coupled cardiomyocytes is devised in this thesis. Detailed numerical simulations are performed to investigate the conditions under which they synchronize. Synchronization is deemed necessary for the successful growth of engineered tissue. Mechanical coupling in the early stages of EHM growth happens via the extracellular matrix (ECM), i.e., the collagen hydrogel (with or without fibroblasts) surrounding the cells. The mechanical properties of these hydrogels with and without fibroblasts and CM were investigated in rheological experiments.

In the numerical simulations of viscoelastically coupled CM, various forms of $n: m$ synchronization - including $n=m=1$ - of the cells are observed if the coupling matrix is sufficiently stiff. This matches qualitatively the regime of viscoelastic parameters into which the ECM is observed to develop in the rheological experiments. In those, the presence of fibroblasts is found to further stiffen the ECM compared to pure collagen or collagen with CM only. Fibroblasts thus improve the conditions for synchronization of CM coupled by the ECM.

The special case of purely elastic coupling, though being unphysiological in the context of tissue engineering, shows interesting antiphase synchronization and chimera behavior in the numerical simulations.
\end{abstract}




\section{Contents}

Glossary and Abbreviations vi vi vis

1 Introduction $\quad 1$

1.1 Research questions and scope of this thesis . . . . . . . . . . . . . 2

1.2 The heart and heart muscle cells . . . . . . . . . . . . . . 3

1.2.1 Heart and heart failure . . . . . . . . . . . . . 4

1.2 .2 Heart muscle cells . . . . . . . . . . . . . . . . 5

1.2.3 Contraction of cardiomyocytes . . . . . . . . . . 7

1.3 Engineering cardiac tissue . . . . . . . . . . . . . . . . . 8

1.4 Collagen . . . . . . . . . . . . . . . . . . . . . . 10

1.5 Oscillation and synchronization . . . . . . . . . . . . . 11

2 Methods $\quad \mathbf{1 3}$

2.1 Modelling viscoelastically coupled cardiomyocytes . . . . . . . . . . 13

2.1.1 Electrical excitation of the membrane voltage . . . . . . . . 13

2.1.2 Active contraction of cardiomyocytes . . . . . . . . . . . . 16

2.1.3 Mechanosensing via stretch-activated ion channels . . . . . . . . 17

2.1.4 Linear viscoelastic coupling . . . . . . . . . . . . . . . 18

2.2 Implementation and analysis . . . . . . . . . . . . . . . . 21

2.2.1 Evaluating modes of synchronization of two oscillators . . . . . 22

2.2.2 Determining phases and measures of synchronization of a linear chain . . . . . . . . . . . . . . . . 23

2.3 Concepts of rheology . . . . . . . . . . . . . . . . . . . . . . 24

2.3.1 Linear rheology . . . . . . . . . . . . . . . . . . . 26

2.3.2 Nonlinear rheology and LAOS . . . . . . . . . . . . . . 28

2.4 Experimental methods and analysis . . . . . . . . . . . . . . . . 30

2.4 The rheometer . . . . . . . . . . . . . . . 30

2.4.2 Collagen hydrogels with and without cells . . . . . . . . . 32

2.4.3 Experimental protocols . . . . . . . . . . . . 33

3 Synchronization of viscoelastically coupled excitable oscillators $\quad 39$

3.1 Dynamics of two coupled cells and of linear chains . . . . . . . . . . . . 39

3.2 Synchronization in short linear chains . . . . . . . . . . . . . . 50 
4 Rheological properties of the extracellular matrix $\quad 53$

4.1 Development of the ECM . . . . . . . . . . . . . . 53

4.2 Nonlinear stress response of collagen . . . . . . . . . . . . . . . 64

4.2.1 Sinusoidal and nonsinusoidal strain input . . . . . . . . . . 65

4.2.2 Ad-hoc model and stress response to higher frequencies . . . . . 66

4.2.3 Prestress measurements . . . . . . . . . . . . . . . . 68

4.3 Linear rheology as a means of collagen comparison . . . . . . . . . . . 70

5 Discussion and outlook $\quad \mathbf{7 5}$

5.1 Simulating viscoelastically coupled excitable oscillators . . . . . . . . 75

5.2 Rheology of the extracellular matrix . . . . . . . . . . . . . . 76

5.3 Outlook: Combining simulations and ECM measurements . . . . . . . . 78

5.4 Conclusion . . . . . . . . . . . . . . . . . 79

$\begin{array}{ll}\text { References } & 81\end{array}$

$\begin{array}{lll}\text { Appendix A Initial conditions and parameters } & 91\end{array}$

A.1 Natural frequencies of individual cells . . . . . . . . . . . . . . . 91

A.2 Initial phases and initial conditions . . . . . . . . . . . . . . . 92

$\begin{array}{lll}\text { Appendix B Ageing of Collagen } & 93\end{array}$

$\begin{array}{ll}\text { Appendix C Coefficients of the ad-hoc model } & 97\end{array}$

C.1 Supplementary material of Schlick et al. 2019 . . . . . . . . . . . . . . 97

C.2 Coefficients used for non-sinusoidal strain input . . . . . . . . . . . 98 


\title{
Glossary and Abbreviations
}

\author{
Abbreviations \\ AFM Atomic force microscope \\ AP Action potential \\ CM Cardiomyocyte, heart muscle cell \\ ECC Excitation-contraction coupling \\ ECM Extracellular matrix \\ EHM Engineered human myocardium, see section 1.3 \\ EOM Equations of motion \\ HFF Human foreskin fibroblasts, Fib in [1] \\ HF Heart failure \\ hiPSC Human induced pluripotent stemcells \\ LAOS Large-amplitude oscillatory shear \\ MEF Mechanoelectric feedback \\ ODE Ordinary differential equation \\ SAC Stretch-activated current \\ SEM Scanning electron microscope \\ SR Sarcoplasmic reticulum
}

\section{Glossary}

action potential An excitation in the membrane voltage of a biological cell (in this work: heart muscle cell), either due to internal dynamics or as a response to an external stimulus.

chimera state A dynamical state in a network of coupled oscillators where coherent and incoherent populations coexist $[2,3]$. 
cardiomyocyte Contracting heart muscle cell the coordinated contraction of which constitutes the pumping function of the heart. The mechanical contraction of the cell is triggered by an action potential via ECC.

collagen Most abundant biopolymer in the human body. Provides the basis for the ECM in EHM production.

fibroblast Nonmyocyte cells present in the heart muscle shaping the ECM by secreting collagen.

gelation/polymerization In the context of the collagen hydrogels used in this thesis, gelation and polymerization denote the formation of a collagen network from the initially solved collagen after neutralization with $\mathrm{NaOH}$. It is visible in a rapid increase in $G^{\prime}$ and $G^{\prime \prime}$ in the time sweep.

Maxwell element A spring-dashpot representation of a linear viscoelastic fluid consisting of a spring and a dashpot in series. Used in the present work to model the ECM.

membrane voltage Difference in electrical potential between inside and outside of biological cells, i.e., potential difference across the cell membrane.

refractory period The period of time following an excitation in which a new excitation is not possible.

strain-stiffening Stronger-than-linear increase of stress with increasing strain.

\section{Symbols}

a Parameter controlling onset and frequency of oscillations of membrane voltage

$d \quad$ Viscous creep

$\Delta x \quad$ Deformation, cell elongation

$\gamma, \dot{\gamma} \quad$ Strain and strain rate

$\gamma_{0}, \dot{\gamma}_{0}$ Amplitudes of oscillatory strain and strain rate

$G^{\prime}, G^{\prime \prime}$ Storage and loss modulus

$I_{\mathrm{S}} \quad$ Stretch-activated current

$k_{\mathrm{c}}, \gamma_{\mathrm{c}}$ Elasticity and fluidity of the Maxwell element representing the ECM

$k_{\mathrm{r}}, \gamma_{\mathrm{r}}$ Stiffness and damping of the internal harmonic relaxation force of the cells

$\psi \quad$ Phase of oscillation

$\sigma \quad$ (Shear) stress

$T \quad$ Active contractile force

$t \quad$ Time

$v \quad$ Normalized membrane voltage 



\section{Chapter 1}

\section{Introduction}

In the human body as in all vertebrates the heart is responsible for pumping blood and thus delivering oxygen and nutrients to all organs [4]. When its function is impaired by sickness or injury, severe consequences can follow. Depending on the level of impairment, they can range from reduced physical performance to heart failure (HF) when the heart is not able to pump a sufficient amount of blood to all organs [5]. While heart diseases in general pose a major cause of death worldwide, heart failure specifically affects over 6.5 million people in the US alone [6]. There are numerous possible treatments to HF, ranging from pharmaceuticals to a transplantation of the whole organ in the most severe, life-threatening cases $[7,8]$. Since the supply of donor organs is limited [9], new therapeutic approaches are needed. One possibility of finding such a new approach is the in-vitro engineering of cardiac tissue [10] from heart muscle cells. Not only does direct implantation of engineered tissue offer the prospect of improving the function of the organ in the case of $\mathrm{HF}$ and ideally regenerating the heart muscle itself $[11,12]$, engineered tissue also offers the perspective of facilitating disease modelling, drug development, and drug testing [13, 14].

The success of engineering cardiac tissue depends on the ideal combination of constituents [15]; not only the heart muscle cells or cardiomyocytes (CM) themselves but also possible nonmyocyte cells (mainly fibroblasts) and the extracellular matrix (ECM) into which the cells are embedded. Understanding how the interactions between the cells lead to the growth of functioning tissue may not only improve the success rates and, ultimately, the yield of tissue engineering but may also help understanding better how the heart as an organ forms in an embryo in nature. CM contract mechanically; in healthy tissue, their synchronized, coordinated contraction constitutes the pumping function of the whole organ [16]. Mechanical contraction in each CM is triggered by an electrical excitation in the membrane voltage of the cell, an action potential, via a process called excitation-contraction coupling (ECC) $[17,18]$. In connected tissue, cross talk between the cells' electrical excitations happens mainly electrochemically via gap junctions formed between neighboring cells [19] but the cells can also influence each other electromechanically via mechanoelectrical feedback [20-22]. When growing engineered tissue, the cells initially oscillate on their own [23]. Exactly how, when and under which conditions the individually beating cells synchronize and whether this 
synchronization is a necessary condition or a consequence of growing into a connected tissue is poorly understood.

On a more abstract level, the question of synchronization in cardiomyocytes is part of the study of dynamics of coupled oscillators. Over the last decades and centuries, there have been many studies on synchronization phenomena in coupled linear and nonlinear oscillators [24]; from the first description of two coupled pendulum clocks $[25,26]$ by Huygens over networks of phase oscillators with diverse types of coupling functions [27-29] to chimera states [2,3,30,31] in networks of various kinds of (identical) oscillators. Despite the large number of studies, the specific system of viscoelastically coupled excitable oscillators describing the CM coupled by the ECM is not present in literature. Even the broader class of viscoelastically coupled non-linear oscillators is not studied often [32], so open questions remain regarding synchronization dynamics in this area.

\subsection{Research questions and scope of this thesis}

The research done in the course of this thesis was conducted as part of the collaborative research center SFB 937, project A 18. The two central hypotheses of this project were that

1. for successful engineering of cardiac tissue from cardiomyocytes, these cells have to synchronize their beating and

2. this synchronization happens due to the mechanical coupling via the extra-cellular matrix in which the CM are embedded.

This thesis concentrates on the second hypothesis. The underlying question to be studied is whether and how synchronization of the electrical excitations of purely mechanically coupled CM occurs qualitatively in numerical simulations. Therefore a mathematical model describing the coupled CM qualitatively as viscoelastically coupled excitable oscillators is devised. Since the results depend on the mechanical properties of the ECM, rheological measurements to determine these properties, both linear and nonlinear, are conducted. Ideally, the results of these measurements can then be used to model the mechanical coupling more realistically and determine ECM parameters for which one expects synchronization of the CM.

On its own, the work in this thesis can not prove any of the two hypotheses listed above since the experimental proof of synchronization and mechanical interactions leading to it is well beyond the scope of my work. In the larger context of the collaboration however, results of the study of synchronization dynamics of viscoelastically coupled CM could in principle falsify the second hypothesis, i.e., synchronization by mechanical coupling via the ECM, especially if no synchronization at all or only at unphysiological parameters would be found. If on the other hand synchronization purely by mechanical coupling, i.e., without direct electrical coupling is found, the second hypothesis is further supported. 


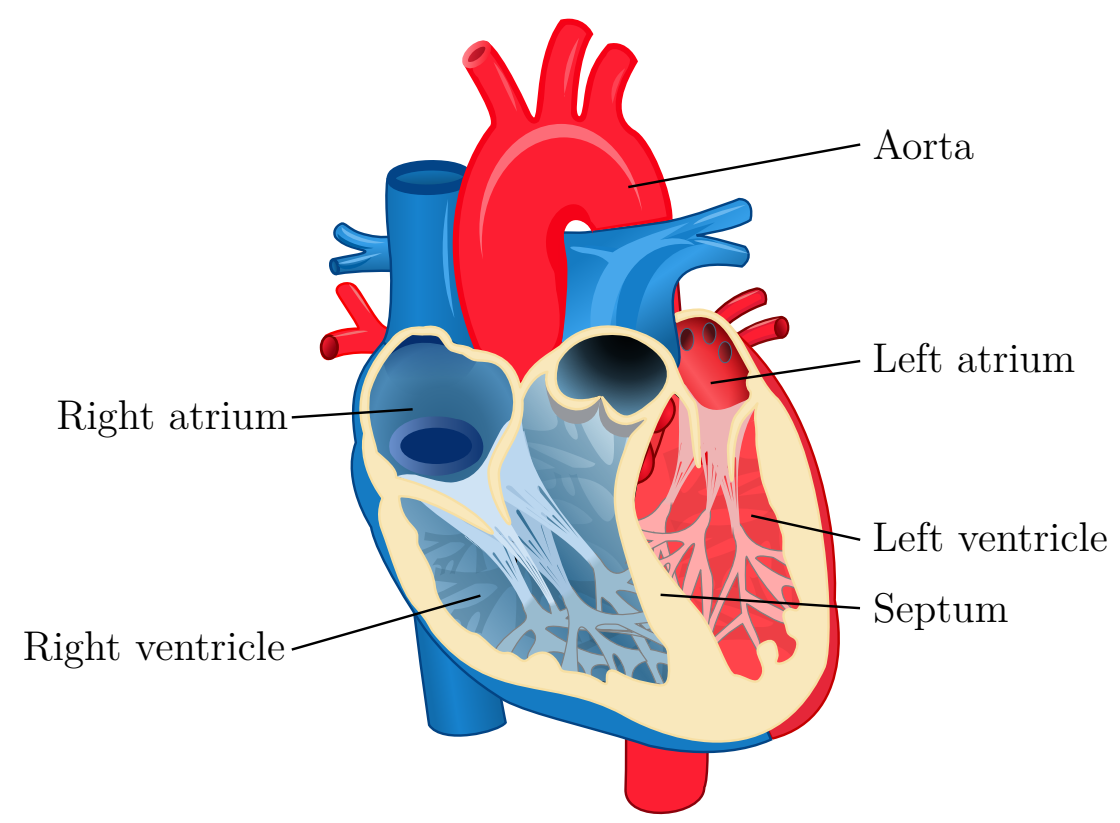

Figure 1.1 Sketch of the human heart with left and right ventricles and atria. Blue and red colored regions indicate deoxygenated and oxygenated blood pathways, respectively. Adapted from [34], Wikimedia Commons, CC BY-SA 3.0 ${ }^{1}$.

The thesis is structured as follows. The coming sections in chapter 1 introduce general concepts of the heart, heart failure, and tissue engineering as well as the phenomenon of synchronization in coupled oscillators. The mathematical model for viscoelastically coupled excitable oscillators and its implementation are introduced in chapter 2 as well as concepts of rheology and the experimental protocols used in measuring the rheological properties of the extracellular matrix. Results of the numerical simulations are presented in chapter 3 , while chapter 4 contains the results of measuring the ECM rheology. Here, the sections 3.1 and 4.1 consist of the two publications [33] and [1], respectively. [33] contains the numerical study of synchronization in systems of two and of linear chains of viscoelastically coupled excitable oscillators. In [1], we investigate the role of fibroblasts and cardiomyocytes on ECM formation and the rheology of the ECM. The discussion in chapter 5 bridges the two parts, simulations and experiments, and presents an outlook on possible future work.

\subsection{The heart and heart muscle cells}

Before introducing the concepts of engineering cardiac tissue it is worth looking in more detail at the organ itself and the cells it is mainly made of. 


\subsubsection{Heart and heart failure}

The heart is one of the most important organs in our bodies. In an average adult it beats around 100000 times per day, pumping around 4 to 8 litres per minute [4]. Its general buildup is sketched in figure 1.1; it is a muscle essentially consisting of four compartments: right and left ventricles and atria, respectively. The two ventricles are separated by the septum. The outer heart wall of the organ mainly consists of the myocardium, the actual heart muscle responsible for the major part of its pumping function [35]. The muscle itself is mainly made out of connected heart muscle cells, cardiomyocytes $(\mathrm{CM})$, that will be presented in more detail in the following section. At each contraction of the muscle, deoxygenated blood is pushed out of the right ventricle to the lungs and oxygenated blood flows from the left ventricle into the rest of the body. After contraction the muscle dilates and the ventricles are filled again with oxygenated blood from the lungs and deoxygenated blood from the rest of the body.

Each mechanical beat is preceded and triggered by an electrical excitation of the myocardium. There are specific pacemaker regions in the organ that are responsible for the initiation of this electrical activity and thus keeping up the healthy cardiac rhythm. The origin of the electrical activity is the sinoatrial (SA) node located at the top of the right ventricle [36]. From there, the excitation is forwarded through the atria to the atrioventricular (AV) node located at the septum between the atria where it is delayed to allow for full contraction of the atria. The excitation then continues through atrioventricular bundles, bundle branches, and so-called Purkinje fibers to the ventricular myocardium where it triggers the actual contraction [35]. Many cardiac diseases and arrhythmias can be linked to faulty conduction or excitation dynamics $[37,38]$.

Due to its general importance to the functioning of the body, pathological impairments of the heart by disease or injury can pose a major health risk. Almost consequently, cardiovascular diseases in general are the major cause of death worldwide [39]. One of the most widespread is heart failure (HF) whereby the pumping function of the heart is reduced to a level that it cannot deliver enough oxygen to the rest of the body [8]. In the US alone, in total 6.5 million people are affected by this condition [6]; in Germany, HF is the most common reason for hospitalization [40]. Especially when it becomes chronic, the majority of cases of HF can be associated with myocardial remodeling, fibrosis and, ultimately, cardiomyocyte death [41]. Most current treatments of HF can only soften its symptoms or slow down the remodelling process, but there is currently no therapy available that actually restores CM function [42] - the exception being the full transplantation of the organ. Implanting engineered tissue provides a prospect of remuscularization and thus a possible cure of the underlying cause to $\mathrm{HF}$ [43]. A more comprehensive discussion of tissue engineering will be given in section 1.3. 


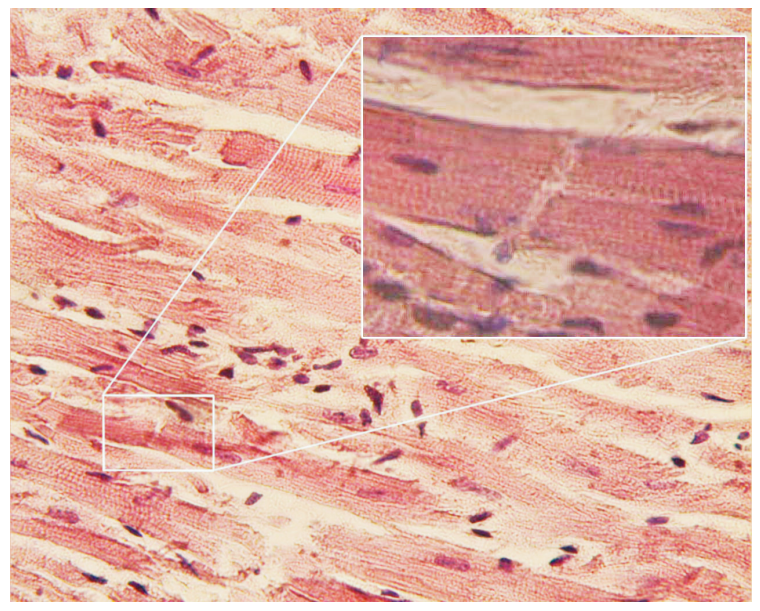

(a) Cardiac muscle tissue

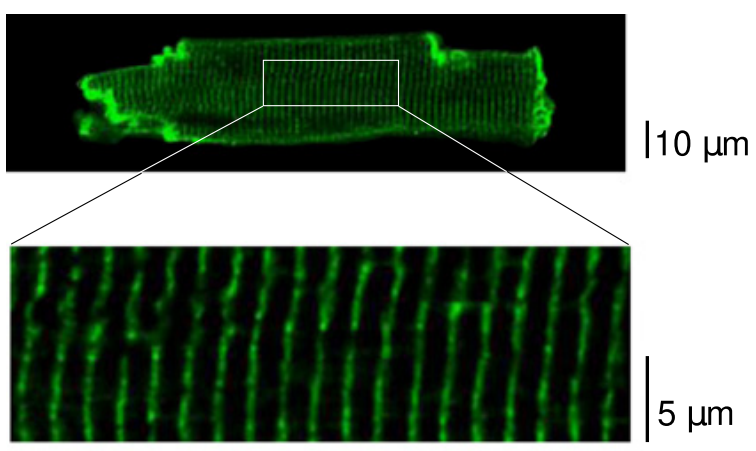

(b) Z-bands of an isolated CM

Figure 1.2 Images of connected and isolated cardiomyocytes. (a) Myocardial tissue with CM (darker red) and ECM (lighter regions). The CM are connected by intercalated discs (highlighted). Inside the CM, the myofibrils responsible for contraction are visible. Image taken from [44], Wikimedia Commons, CC BY-SA 3.0. (b) Confocal image of a CM in which cell membrane and Z-bands have been made visible using a fluorescent dye (di-8-ANNEPS). Image taken from [45] with permissions.

\subsubsection{Heart muscle cells}

As mentioned previously, the contractile function of the myocardium is generated by its heart muscle cells or cardiomyocytes (CM). However, those are not the only cells that make up the myocardium - in fact about $30 \%$ of all cells are CM [46]. The largest part of the remaining cells are endothelial cells (about $45 \%$ ) and fibroblasts $(\gtrsim 10 \%)$ that are mainly responsible for providing an ideal mechanical environment for the CM. Together with the surrounding collagen fibers, the nonmyocyte cells make up the extracellular matrix in healthy adult cardiac tissue that is shown in figure 1.2a. The almost rectangular-shaped CM are largely aligned. Since they contract along their long axis, this alignment provides a direction of contraction for the whole tissue. The contraction of the individual cell involves myofibrils inside the cell and will be explained in section 1.2.3. The cells are bounded by their cell membranes which is disrupted by a regular pattern of Z-bands that are shown in figure $1.2 \mathrm{~b}$. They - or, more precisely, structures called T-tubuli within the Z-bands - allow for an additional exchange of ions between intra- and extracellular space also for the intracellular regions further apart from the cell membrane.

As is the case in most biological cells, there is an electrical voltage across the membrane of CM, the membrane potential. It is defined as the difference in electrical potential between the inside of the cell and the extracellular medium stemming from different ion concentrations inside and outside the cell [47]. At rest, the ion concentrations are constant and determined by the permeability of the cell membrane for

\footnotetext{
${ }^{1}$ https://creativecommons.org/licenses/by-sa/3.0/deed.en
} 


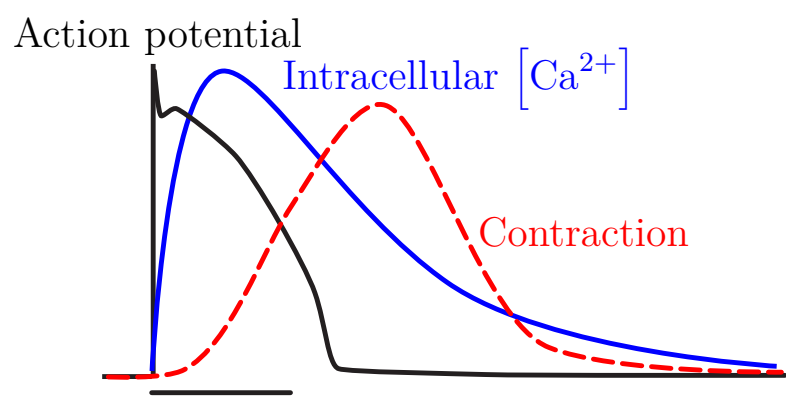

$200 \mathrm{~ms}$

Figure 1.3 Sketch of a cardiac action potential, the intracellular calcium concentration and the contraction of a CM. The excitation in the membrane voltage, i.e., the action potential, triggers the calcium dynamics that in turn start the contraction mechanism of the cell. Adapted with permission from [18].

the individual ion species. The membrane voltage can then be calculated via Nernst's equation. In this state, the membrane voltage of a human $\mathrm{CM}$ is about $-80 \mathrm{mV}$. The ion species that contribute most to the cardiac membrane voltage are $\mathrm{Na}^{+}$and $\mathrm{Ca}^{2+}$ whose concentrations inside the cell are lower at rest than outside, and $\mathrm{K}^{+}$that has a higher concentration at rest inside than outside the cell.

The permeability of the cell membrane however, can change drastically by the opening of ion channels that either passively allow ions to pass through when open or actively pump ions through the membrane. For an extensive review on ion channels in CM see, e.g., [48]. Most of these channels open in response to a change in membrane voltage leading to the most important feature of the electrical dynamics of a $\mathrm{CM}$, the action potential (AP) that is sketched in figure 1.3 in black. After an initial slight increase in membrane voltage above $-70 \mathrm{mV}$, voltage gated sodium channels open and $\mathrm{Na}^{2+}$ is rapidly transported into the cell, leading to the fast upstroke or depolarization of the membrane voltage [47] to values around $20 \mathrm{mV}$ in a time span of about 10 ms. After a short decrease in membrane voltage (initial repolarization) caused by a transient outflow of $\mathrm{K}^{+}$triggered by the increase in membrane voltage, the slower calcium dynamics take over. Through the membrane and the Z-bands, Ca2+ enters the cell where it triggers a further calcium-induced calcium release from separated reservoirs inside the cell called sarcoplasmic reticulum (SR) [17]. Outward $\mathrm{K}^{+}$and inward $\mathrm{Ca}^{2+}$ (both from the outside of the cell and the SR) currents approximately balance each other leading to a prolonged plateau phase in the AP. By the opening of additional slower potassium channels, the membrane voltage decreases further during repolarization until it reaches its resting value again. At the same time, active ion channels exchange $\mathrm{K}^{+}$and $\mathrm{Na}^{+}$through the cell membrane and $\mathrm{Na}^{+}$and $\mathrm{Ca}^{2+}$ through membrane and into the SR, thus restoring the initial ion concentrations. Even after the membrane voltage has returned to its resting value, some ion channels remain in an inactivated state from which they cannot open again. This leads to a period of time after the AP, called refractory period, in which the cell does not exhibit an AP even when the membrane voltage is increased above the threshold of the opening of 
the sodium channels. Only when these channels have returned to their resting, closed state, a new AP can be triggered.

The whole dynamics of the membrane voltage can be described as an excitable system [49]: While a small distortion from the resting state, i.e., increasing the membrane voltage to values below the opening threshold of the sodium currents will just decay to the resting state, a hyperthreshold stimulus triggers an excitation, i.e., an action potential. As long as the stimulus is large enough to overcome the threshold, its exact strength does not matter - it will in any case trigger the AP dynamics described above and thus cause an excitation. The excitation then is followed by a period of time in which no new excitation is possible - the refractory period.

There is another type of ion channels not mentioned before but relevant to this thesis - channels gated by mechanical stimuli such as stretch of the cell membrane or change in cell volume $[50,51]$. When these channels open due to a stretch of the cell, they cause an inflow of positive ions, that in turn may cause the membrane voltage to exceed the excitation threshold and thus cause an AP. This influence of a mechanical stimulus on the electrical dynamics of the cell is called mechanoelectric feedback (MEF) [20].

In the healthy myocardium, cardiomyocytes are coupled electrically to their neighbors by gap junctions [19]. They allow for the exchange of ions between the cells whereby an AP in one cell causes the membrane voltage in the neighboring cells to exceed the excitation threshold as well. If they are not refractory due to an earlier excitation, they exhibit an AP. This leads to the propagation of the excitation through the whole tissue, completing the picture of the conduction of the electrical activation through the myocardium introduced in section 1.2.1. After having entered the myocardium through the Purkinje fibers close to the apex, i.e., the lower end of the heart, the excitation travels upwards through the ventricular myocardium, thus recruiting the $\mathrm{CM}$ in the myocardium in a coordinated way. This propagation of excitation allows for the description of cardiac tissue as an excitable medium [49].

\subsubsection{Contraction of cardiomyocytes}

The electrical dynamics of the membrane voltage of a cardiomyocyte discussed above have the specific task to trigger the generation of a contractile force in each individual $\mathrm{CM}$. The alignment of the CM in the tissue (cf. figure 1.2a) leads to the coordinated contraction of the whole organ and thus generates its pumping function. The process whereby the electrical excitation triggers mechanical contraction is called excitationcontraction coupling (ECC) [18]. A key element in this process is the intracellular calcium concentration that is strongly increased by the calcium-induced calcium release from the SR caused by the action potential. Its time trace following the onset of an $\mathrm{AP}$ is sketched in figure 1.3 in blue.

The increase in intracellular $\mathrm{Ca}^{2+}$ concentration triggers the contraction machinery in the myofibrils inside the CM. The myofibrils consist of complexes of actin filaments and myosin, actomyosin complexes, organized in subunits called sarcomeres [52] located between the Z-lines depicted schematically in figure 1.4. In the presence of calcium 


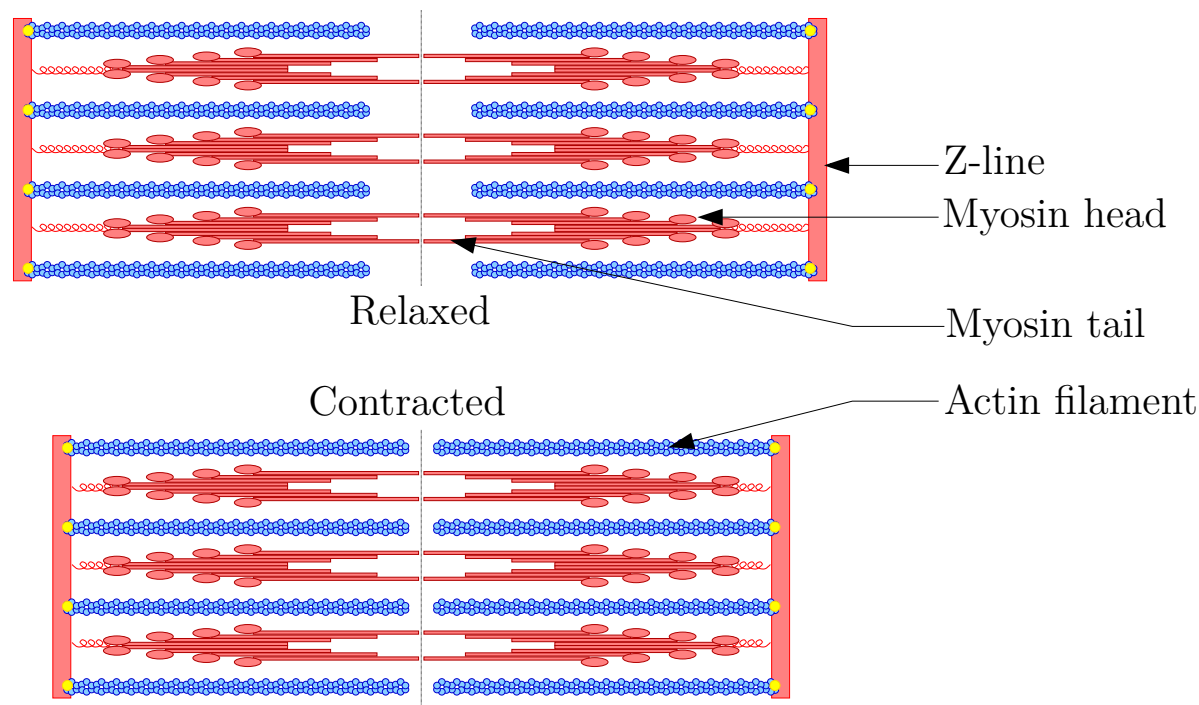

Figure 1.4 Schematic depiction of a sarcomere in relaxed and contracted state. An increase in available $\mathrm{Ca}^{2+}$ leads to the binding of myosin heads to the actin filaments. This pushes the myosin along the actin and thus, contracts the sarcomere. Adapted from [52], Wikimedia Commons, CC BY-SA 3.0.

ions, myosin heads bind to the actin filaments whereby the whole myosin complex moves into the cavities spanned by the actin [53]. As long as there is calcium available, this leads to even more available actin binding sites for the myosin heads and thus, to a positive feedback resulting in a force generated by the sarcomere that pulls the two Z-lines together. When this happens simultaneously in all sarcomeres in one cell, as is the case when the calcium release is triggered by an action potential, the whole cell contracts. This contraction as a response to the increase in intracellular calcium concentration is shown in figure 1.3 in red. The need for a coordinated contraction of all sarcomeres is why the calcium-induced calcium release from the SR is so important not only at the outer cell membrane but also along the Z-lines (or, more specifically, the T-tubuli) [53]. It allows for an increase in local $\mathrm{Ca}^{2+}$ concentration simultaneously at actomyosin complexes close to and further away from the cell membrane. When the calcium dynamics is impaired, the contractility of a CM is reduced which can in turn lead to a reduced pumping performance and thus, heart failure on the organ level [45].

Together with the MEF, the contraction of CM provides another pathway of cellto-cell cross talk besides the coupling via gap junctions. One cell's contraction can deform its neighbors and influence their electrical dynamics via $\mathrm{MEF}$ - in case of large deformations it may even depolarize these cells [21].

\subsection{Engineering cardiac tissue}

After having introduced the general function and features of the heart muscle and its cells we can now turn to the engineering of cardiac tissue in vitro. As explained 

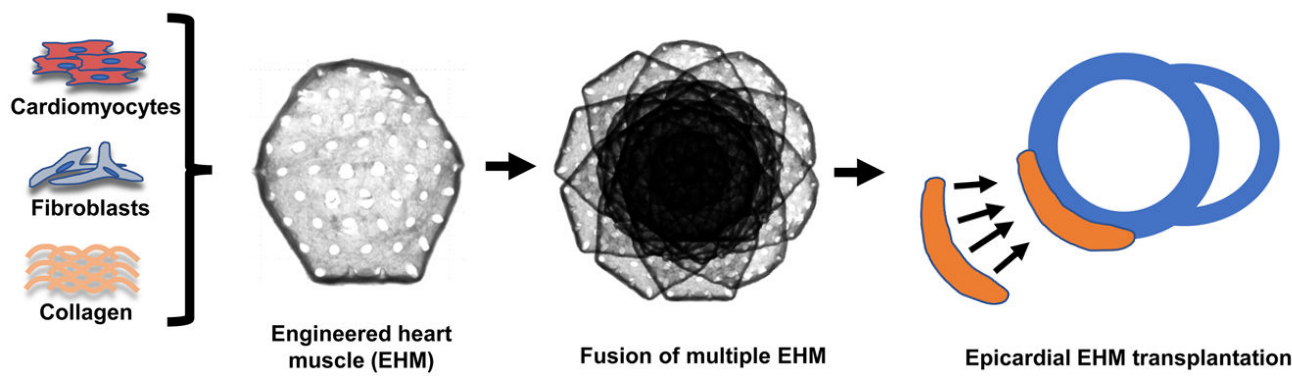

Figure 1.5 Idea of engineering and transplanting cardiac tissue. The tissue is grown from CM in an ECM (here: fibroblasts and collagen). After possibly fusing several patches of engineered tissue to form a larger and thicker compound, it is implanted on the myocardium to improve contractility and ideally remuscularize damaged regions. Adapted with permission from [43].

above, engineered tissue offers the prospect of regenerating the myocardium in the case of heart failure without having to transplant the whole organ. The conceptual idea of in-vitro engineering and implanting tissue is sketched in figure 1.5. Even without the direct therapeutic application, engineered tissue can serve as a model in drug development and testing [13].

Beginning in 1997 with embryonic chick cardiomyocytes, Eschenhagen et al. [54] started culturing CM in a three-dimensional collagen matrix, resulting in coherently contracting model tissue. This was later extended by Zimmermann et al. [55, 56] to rat $\mathrm{CM}$ in a collagen matrix, producing contracting rings basing on mammalian instead if avian CM, thus giving the first perspectives of engineering heart tissue for therapeutic purposes. The next steps towards clinical application included the usage of human induced pluripotent stem cell (hiPSC) derived CM instead of animal cells $[15,57]$. There are also alternatives to using collagen for the ECM, e.g., fibrin [58]. For the engineered tissue derived from hiPSC CM and collagen type I, Zimmermann et al. coined the term "engineered human myocardium" (EHM). These tissues were used to graft the patches shown in figure 1.5 that have areas of the order $\mathrm{cm}^{2}$ each. The general protocol starts out from hiPSC CM, type I collagen, and human foreskin fibroblasts $(\mathrm{HFF})$ that over the course of several weeks grow into contracting rings or patches. The contractile force of these rings or patches corresponds to that of cardiac tissue in an infant [15]. Compared to the initial hiPSC CM being more similar to embryonic CM, that implies an advanced degree of maturation during the growth of the EHM. In preclinical studies EHM has already been successfully implanted into rats $[15,43,57]$ and macaques [12].

With the clinical application in mind, induced pluripotent stem cells could even allow for patient-specific autografts, i.e., patients being treated with EHM derived from CM that in turn stem from their own reprogrammed somatic cells. However, due to the large number of possible patients, the cost and time required for reprogramming, and the risk of unwanted mutations, autografts seem unlikely in clinical application 


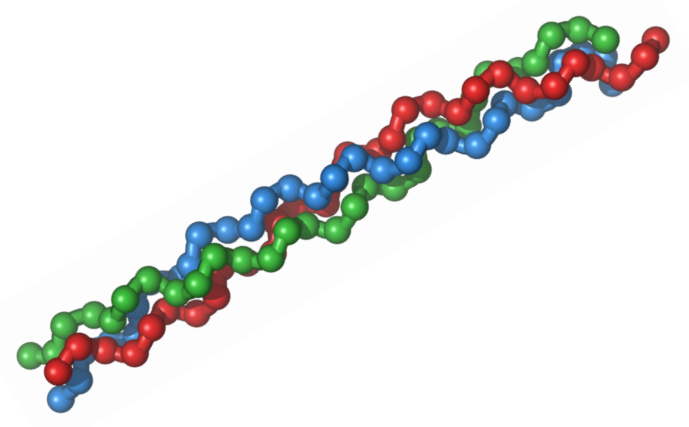

(a) Tropocollagen triple helix

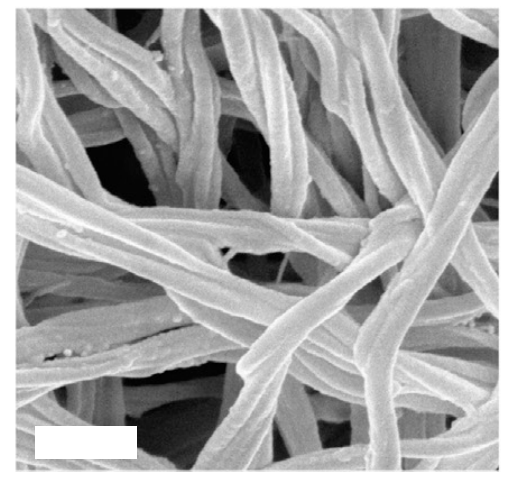

(b) SEM image of collagen network

Figure 1.6 Collagen consists of cross-linked tropocollagen molecules (a) that are built of a triple helix of three strands of procollagen. Image taken from [66], Wikimedia Commons, CC BY-SA 3.0. These molecules form microfibrils and fibrils that arrange into the collagen network. (b) Scanning electron microscope (SEM) image of a collagen network in vitro at a temperature of $30{ }^{\circ} \mathrm{C}$ and a concentration of $4 \mathrm{mg} / \mathrm{ml}$. The scale bar in the lower left represents $200 \mathrm{~nm}$. Image reprinted from [67] with permission.

[11]. This means that, although basing on hiPSC, implants of engineered tissue would still need to be accompanied by immune suppression comparable to conventional wholeorgan transplants. Apart from implantation, EHM and engineered tissue in general can facilitate drug development and testing [13]: being derived from human cells, both the reaction to drugs and disease modelling in EHM could be closer to those of humans than are, e.g., animal models. At the same time, EHM provides an higher grade of maturation than conventional cell cultures.

Alternatives to engineering functioning myocardium include the grafting of cell sheets of hiPSC CM without letting the cells grow into contracting tissue [59, 60] or direct implantation of stem cells or CM [61,62]. There is also the approach of reprogramming fibroblasts to CM-like cells in hearts affected by HF to improve pumping function [63].

\subsection{Collagen}

Collagen is the most abundant biopolymer found in mammals and accounts for about $30 \%$ of the total protein mass [64]. There are 28 different types of collagen [65], usually labeled with Roman numerals I - XXVIII. Of these, collagen type I is by far the most common in the human body - $90 \%$ of the collagen found in humans is of type I. Mainly for this reason, it is also the type of collagen used for EHM and thus in the experiments conducted in the course of the present thesis, too. As mentioned above, the collagen I provides the basis for the ECM of engineered human myocardium [57]. In the living organism it provides structured networks found in the extracellular matrix of muscle tissue, in the skin, bones, and tendons. 
Type I collagen has a highly hierarchical structure. On molecular level it consists of tropocollagen - a molecule that itself consists of three procollagens arranged in a triple helix as sketched in figure 1.6a [68]. These molecules in turn are connected by crosslinkers and form microfibrils that align and connect to form the collagen fibrils. Those, finally, arrange into the network structure shown in figure $1.6 \mathrm{~b}$ that is observed in vitro and in vivo. For a more detailed explanation of the hierarchical structure and its chemical components, see $[65,67,68]$. The thickness, length, and strength of the individual microfibrils and fibrils and thus, the overall network depends on external conditions such as wetness, $\mathrm{pH}$, and temperature $[69,70]$. In the body, collagen - or procollagen, to be precise - is secreted by fibroblasts [64] highlighting the importance of fibroblasts for the functioning of the ECM.

\subsection{Oscillation and synchronization}

Since a large part of the present thesis will treat the dynamics of coupled periodically oscillating cardiomyocytes it is worthwhile to introduce the phenomenon of synchronization in interacting oscillators in general. This is a broad and complex topic and has been the object of research for decades to centuries [25, 26, 71-75]. Thus the following brief introduction can by no means cover all aspects. For a more detailed introduction see e.g. [74, 75]. The term synchronization stems from the Greek and can be translated to "at the same time" [74]. In general, synchronization means that in a system of at least two interacting subunits, the dynamics of some or all of these subunits are changed in speed and frequency by the interaction such that they all express a common temporal behavior. To put it in the words of Balanov et al. [75] for systems of oscillators:

"Synchronization is an adjustment of the time scales of oscillations due to interaction between the oscillating processes."

This can also describe the adjustment of a single oscillator to an external driving. Thus, two oscillators, possibly each with its own amplitude and frequency, are considered synchronous if they express a common frequency when coupled because of the interaction via the coupling.

Oscillations occur in many systems and can have almost arbitrary form, from a simple pendulum to the contracting cardiomyocytes that are the subject of the present thesis to the motion of planets around a star. Often, oscillations are characterized by their phase $\psi(t) \in[-\pi, \pi)^{2}[76]$. In the case of a simple sine $\sin (\omega t)$ with angular frequency $\omega$, the phase is simply ${ }^{3} \psi(t)=(\omega t \bmod 2 \pi)-\pi$. Its time derivative, the phase velocity, $\dot{\psi}(t)=\omega$ is constant. During one period of the sine, the phase grows linearly from $-\pi$ to $\pi$, thus describing the location of the oscillator along the course of its oscillation. This is generalized to arbitrary oscillations such that $\psi(t)$ is a

\footnotetext{
${ }^{2}$ Often, $\psi \in[0,2 \pi)$ is used instead. I use the interval $[-\pi, \pi)$ solely for the reason of consistency with [33]

${ }^{3} \pi$ is subtracted to match the interval above.
} 
monotonously increasing function growing from $-\pi$ to $\pi$ during one period of oscillation. This generalization can still be used if the period itself is not constant anymore. Exactly how that function is defined depends on the system under consideration. For all systems, this function can be chosen such that the resulting phase velocity is constant [74], so $\dot{\psi}=\omega$ as for the simple sine. In the following I will limit all considerations to this case of constant phase velocity. Note that the phase only encodes the temporal component of the dynamics but not shape or amplitude of the oscillations.

Still, the phase is a useful tool when describing the synchronization of oscillators $[74,75]$. Consider two oscillators with constant phase velocities $\dot{\psi}_{1}^{0}=\omega_{1}$ and $\dot{\psi}_{2}^{0}=\omega_{2}$ when both are unperturbed. When these oscillators are coupled they influence each other's (phase) dynamics. As a consequence, they display phase velocities $\dot{\psi}_{1}^{\mathrm{c}}$ and $\dot{\psi}_{2}^{\mathrm{c}}$ that may differ from the unperturbed ones and are, in general, not necessarily constant anymore. In the most strict case, synchronization means that both oscillators, after possible transients, agree on a common frequency $\Omega$ due to the coupling and

$$
\dot{\psi}_{1}^{\mathrm{c}}=\dot{\psi}_{2}^{\mathrm{c}}=\Omega \text {. }
$$

Their phases then evolve with the same velocity and the phase shift

$$
\Delta \psi:=\psi_{1}-\psi_{2}=\text { const }
$$

remains constant over time. In the special case of $\Delta \psi \approx 0$, one speaks of in-phase synchronization, if $\Delta \psi \approx \pm \pi$, of antiphase synchrony. Further, there is the case of $n: m$ synchronization whereby, again due to the coupling,

$$
\frac{\dot{\psi}_{1}^{\mathrm{c}}}{\dot{\psi}_{2}^{\mathrm{c}}}=\frac{n}{m}, \quad n, m \in \mathbb{N} .
$$

Thus, after the first oscillator has advanced by $n$ oscillations, the second has advanced by $m$ oscillations. The above case in equation 1.1 corresponds to $n=m=1$. In a more general and less strict picture of synchronization, equations 1.1 and 1.3 only have to hold for the time-average of the phase velocities over a suitable number of oscillations [74]. This latter concept of a fixed ratio $n: m$ of the averaged frequencies effectively is the one I will use in the course of this thesis (see section 2.2). Incidentally, in the case of $n=m=1$, this corresponds to a true equality of the phase velocities for the right choice of the phases in all my systems.

The concept of synchronization can be extended even to chaotic subunits that - by interaction - synchronize their temporal dynamics even though the synchronized state may still be chaotic [72]. An interesting interplay of synchrony and chaos happens in the case of chimera states $[2,3]$ that have been investigated for almost two decades. Named after an ancient Greek mythological creature consisting of the parts of several different animals, they denote dynamical states of coupled identical oscillators in which synchronous or coherent and asynchronous or incoherent populations coexist [77]. 


\section{Chapter 2}

\section{Methods}

The following chapter introduces the methods used in this work. The mathematical model and its numerical implementation are explained and an overview over the experimental setup and protocol is given. Both are described in sections 3.1 and 4.1 or in the two corresponding publications [33] and [1], too, but less detailed and, in the case of the experimental methods, without the changes to the protocol made after September 2018. Nevertheless, some redundancy cannot be avoided.

\subsection{Modelling viscoelastically coupled cardiomyo- cytes with excitation-contraction coupling}

To model the system of beating cardiomyocytes coupled mechanically by a viscoelastic extracellular matrix one needs mathematical descriptions for each of the individual building blocks: electrical excitation, mechanical contraction, mechanosensing, and mechanical coupling via the ECM. The electrical excitation drives the mechanical contraction which deforms the ECM, thus providing a mechanical interaction that couples the electrical dynamics of the cells via mechanoelectric feedback. The goal of the model is to include all these contributions in a meaningful manner while remaining as simplistic as possible. In the following sections I describe the mathematical models for each part separately, and explain how they are combined to describe the full system.

\subsubsection{Electrical excitation of the membrane voltage}

The electrical excitation of the membrane voltage (see section 1.2.2) can be described in almost any level of complexity. The existing mathematical models range from cellular automata [78] and simplified models with few dynamical variables [79-81] to detailed descriptions of the individual ion currents and concentrations [82-84]. Essentially, all ${ }^{1}$

\footnotetext{
${ }^{1}$ Except for cases like [78] where the dynamics is simplified into a cellular automaton with discrete states.
} 


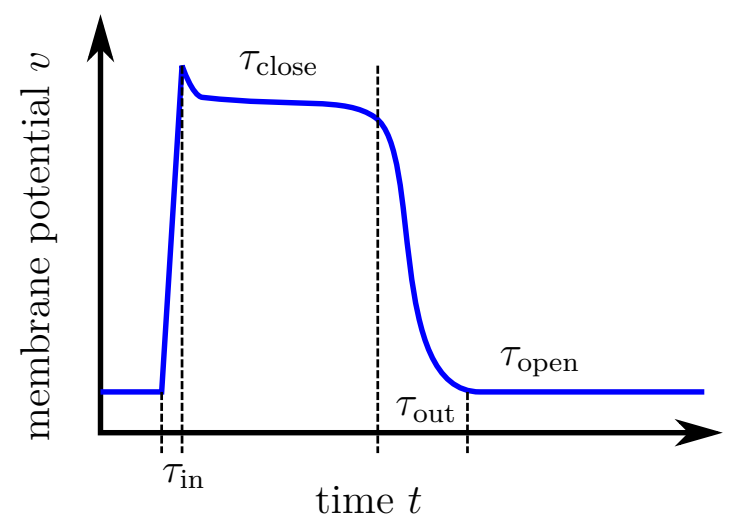

Figure 2.1 Sketch of a cardiac action potential with indicated time scales $\tau_{\text {in }}, \tau_{\text {close }}, \tau_{\text {out }}$, and $\tau_{\text {open. }}$. Reproduced from [85].

models have in common the form of the ODEs describing the dynamics:

$$
\begin{aligned}
\frac{\mathrm{d} v}{\mathrm{~d} t} & =f(v, \mathbf{h}), \\
\frac{\mathrm{d} \mathbf{h}}{\mathrm{d} t} & =\mathbf{g}(v, \mathbf{h})
\end{aligned}
$$

where $v$ denotes the (possibly normalized) membrane voltage and $\mathbf{h}$ is a vector of auxiliary variables describing, e.g., the refractoriness of the cell or the gating of individual ion channels. Often, the function $f(v, \mathbf{h})$ has the form

$$
f(v, \mathbf{h})=\frac{1}{C_{\mathrm{m}}} \sum_{\text {ion }} I_{\text {ion }}(v, \mathbf{h})
$$

where the $I_{\text {ion }}$ are - depending on the model complexity - generalized or actual ion currents through the cell membrane and $C_{\mathrm{m}}$ is the capacitance of the membrane.

Another common feature of most of the mathematical models for the membrane voltage is that they usually do not express oscillatory behavior since they usually describe the dynamics of CM in the whole organ where only specific pacemaker cells exhibit oscillations. On the contrary, the hiPSC derived CM in EHM show pacemaker behavior, i.e., they do oscillate $[15,23]$ with frequencies about $1 \mathrm{~Hz}$. Since that is the system motivating my study of viscoelastically coupled excitable oscillators I use the Mitchell-Schaeffer model [85] in a modified version introduced by Djabella, Landau and Sorine [86] to model the electrical excitation in the following.

Originally, the Mitchell-Schaeffer model was introduced as a special case of a model introduced by Karma [87]. It consists of two dynamical variables; one variable for the normalized membrane potential $v$ and one generalized gating variable $h$. In the 
Mitchell-Schaeffer model, using $C_{\mathrm{m}}=1$, equation 2.3 has the form

$$
\begin{array}{rlrl}
f(v, h) & =I_{\text {in }}(v, h)+I_{\text {out }}(v)+I_{\text {stim }}(t) & \text { with } \\
I_{\text {in }} & =\frac{h v^{2}(1-v)}{\tau_{\text {in }}} & & \text { and } \\
I_{\text {out }} & =-\frac{v}{\tau_{\text {out }}} . & &
\end{array}
$$

$I_{\text {stim }}(t)$ denotes a possibly time-dependent (external) stimulus on the membrane voltage. $\tau_{\text {in }}$ and $\tau_{\text {out }}$ are the timescales of the upstroke and the decrease of an action potential, respectively. Both are sketched in figure 2.1. The gating behavior of $h$ becomes apparent in equation $2.5 ; I_{\text {in }}$ is fully present when $h=1$ and absent if $h=0$. The dynamics of $h$ is governed by the ODE

$$
\frac{\mathrm{d} h}{\mathrm{~d} t}= \begin{cases}\frac{1-h}{\tau_{\text {open }}} & \text { if } v<v_{\text {gate }} \\ \frac{-h}{\tau_{\text {close }}} & \text { else }\end{cases}
$$

where $v_{\text {gate }}$ corresponds to the excitation threshold. $\tau_{\text {open }}$ and $\tau_{\text {close }}$ are time scales of the refractory period and the plateau phase of the AP, respectively. Again, they are depicted in the sketch in figure 2.1. The resting state of this model is $v=0, h=1$; at full excitation $v=1$.

Djabella et al. modified these equations to also allow for oscillatory solutions [86] by introducing parameters $a$ and $\lambda$ to equation 2.5 such that

$$
I_{\text {in }}(v)=\frac{h}{\tau_{\text {in }}}(v+a)(v+a-\lambda)(1-v) .
$$

Tuning $a$ controls the onset and frequency of oscillations or pacemaker activity, $\lambda$ serves to further control excitability. They further regularize the ODE for $h$ by introducing the parameter $\eta_{\text {gate }}$ and functions

$$
\begin{array}{rll}
\epsilon(v, h) & =\frac{1}{\tau_{\text {close }}}+\frac{\tau_{\text {close }}-\tau_{\text {open }}}{\tau_{\text {close }} \tau_{\text {open }}} h_{\infty}(v) & \text { and } \\
h_{\infty}(v) & = \begin{cases}\frac{1}{2}\left[1-\tanh \left(\frac{v-v_{\text {gate }}}{\eta_{\text {gate }}}\right)\right] & \text { if } \eta_{\text {gate }} \neq 0 \\
\frac{1}{2}\left[1-\operatorname{sgn}\left(v-v_{\text {gate }}\right)\right] & \text { if } \eta_{\text {gate }}=0\end{cases}
\end{array}
$$

Here, $\operatorname{sgn}(\cdot)$ is the sign function. The full set of ODEs then reads

$$
\begin{aligned}
\frac{\mathrm{d} v}{\mathrm{~d} t} & =\frac{h}{\tau_{\text {in }}}(v+a)(v+a-\lambda)(1-v)-\frac{v}{\tau_{\text {out }}}+I_{\text {stim }}(t), \\
\frac{\mathrm{d} h}{\mathrm{~d} t} & =\epsilon(v, h)\left(h_{\infty}(v)-h\right) .
\end{aligned}
$$

Equations 2.11 and 2.12 will be used in the following to model the membrane voltage. As Djabella et al. are doing, I will drop the parameter $\lambda$ in the equation of $v$ for 
simplicity, i.e., $\lambda=0$.

\subsubsection{Active contraction of cardiomyocytes}

In principle, modeling the active contraction of cardiomyocytes realistically, requires a detailed description of the intracellular calcium dynamics (see section 1.2.3) in addition to the dynamics of the membrane voltage $[88,89]$. Since firstly, in the course of this thesis, I am mainly interested in a qualitative description and secondly, the calcium release itself is triggered by an action potential, I will use the simplified expression for the active contractile force from Weise and Panfilov [90, 91] directly depending on the (normalized) membrane voltage $v$ introduced in the previous section. The active contractile force $T$ obeys the ODE

$$
\frac{\mathrm{d} T}{\mathrm{~d} t}=\epsilon_{\mathrm{T}}\left(k_{\mathrm{T}} v-T\right)
$$

with parameters $\epsilon_{\mathrm{T}}, k_{\mathrm{T}}>0$. Since $0 \leq v \leq 1, T \geq 0$ at all times. $T$ thus only accounts for the active contraction and has to be countered by a force restoring the cell to its original elongation. In the following, a simple harmonic restoration force

$$
F_{\mathrm{r}}=-k_{\mathrm{r}} \Delta x-\gamma_{\mathrm{r}} \frac{\mathrm{d} \Delta x}{\mathrm{~d} t}
$$

with stiffness $k_{\mathrm{r}}$ and damping $\gamma_{\mathrm{r}}$ will be used. $\Delta x$ denotes the cell elongation.

I can now reduce the actual contraction of the whole cell to the motion of a single point representing the position of the cell membrane as sketched in figure 2.2a. With its position $x$ and resting position $x_{0}$, the cell elongation is

$$
\Delta x=x-x_{0} .
$$

Then the equation of motion (EOM) of the point with (virtual) mass $m$ in terms of $\Delta x$ becomes

$$
m \frac{\mathrm{d}^{2} \Delta x}{\mathrm{~d} t^{2}}=-T+F_{\mathrm{r}}=-T-k_{\mathrm{r}} \Delta x-\gamma_{\mathrm{r}} \frac{\mathrm{d} \Delta x}{\mathrm{~d} t} .
$$

Since $T \geq 0$, it is subtracted on the right hand side; it is a contractile force and the cell elongation should be negative during contraction. Keeping in mind the biological system I want to describe, it is reasonable to consider overdamped motion, simplifying equation 2.16 to

$$
\gamma_{\mathrm{r}} \frac{\mathrm{d} \Delta x}{\mathrm{~d} t}=-T-k_{\mathrm{r}} \Delta x
$$

Together with equations 2.11 and 2.12 for the electrical dynamics and the active contraction in equation 2.13, equation 2.17 describes the full excitation-contraction cycle of a single CM. These equations will be augmented to include the coupling via 


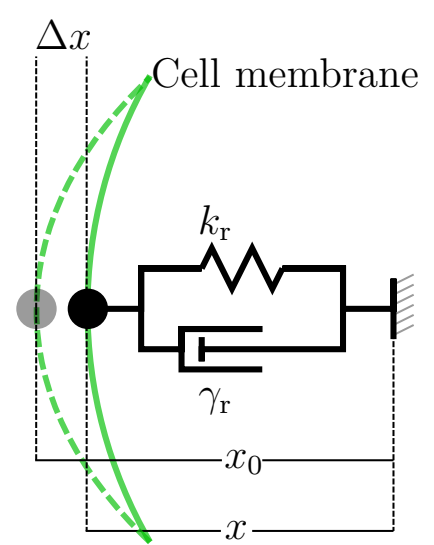

(a) Representation of the cell membrane by a single point.

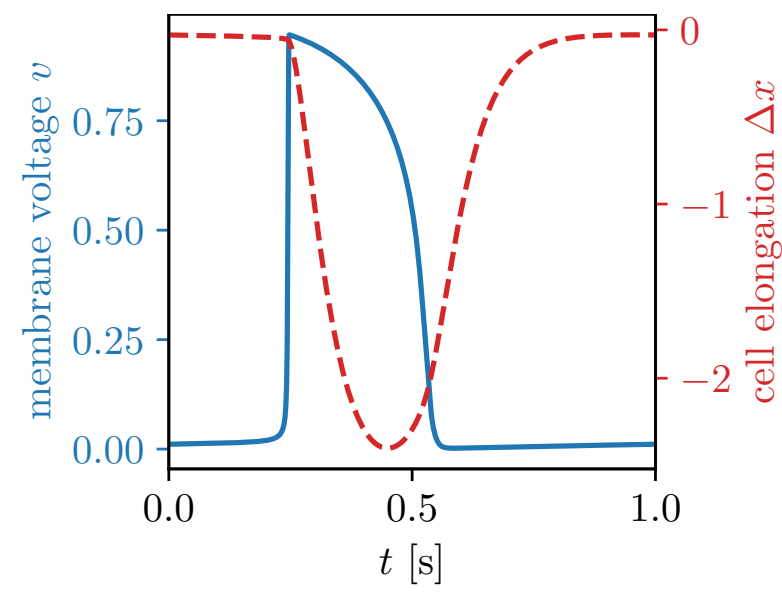

(b) AP and cell elongation of a single cell.

Figure 2.2 Excitation-contraction coupling of a single cell. (a) shows the reduction of the motion of the cell membrane (green) during contraction to that of a single point. The active contractile force $T$ (not shown) is countered by a harmonic restoration represented by a spring with stiffness $k_{\mathrm{r}}$ and a dashpot with damping constant $\gamma_{\mathrm{r}}$. The dashed green line indicates the resting position of the cell membrane. The cell elongation of an isolated single cell follows the AP in the membrane voltage (b). It becomes negative due to the active contraction triggered by the AP and then is restored to the resting state. Integration and model parameters are the same as in section 3.1 and [33]. Note that compared to figure 2 in that section, actual cell elongation is plotted, not contraction, i.e., $-\Delta x$.

the ECM later on. Figure 2.2b shows the time series of the membrane voltage and the cell elongation after integrating the four ODEs with the same parameters that are used in section 3.1. The cell contraction follows the action potential as sketched in figure 1.3 but without calcium dynamics. It becomes negative during contraction and is then restored to its resting state. As intended, there is a clear delay between the maximum of $v$ and peak contraction.

\subsubsection{Mechanosensing via stretch-activated ion channels}

I have now established a model for the excitation-contraction coupling (ECC) of a CM. To account for the influence of mechanical deformations on the membrane voltage of a cell, mechanoelectric feedback has to be included, too. There are numerous ways to exactly model this $[92,93]$, that all result effectively in a way to depolarize the cell and trigger an action potential by a sufficiently large mechanical deformation. Since I am interested in a qualitative description of the mechanoelectric feedback (MEF) in the course of this thesis, I will use the model for an ion current through stretch-activated ion channels in the cell membrane introduced by Nash, Weise, and Panfilov [89, 91, 94] to describe a stretch-activated current (SAC) $I_{\mathrm{S}}$ depending on cell elongation $\Delta x$ and 
membrane voltage $v . I_{\mathrm{S}}$ follows the equation ${ }^{2}$

$$
I_{\mathrm{S}}= \begin{cases}-G_{\mathrm{S}}\left(v-E_{\mathrm{S}}\right) \Delta x & \text { if } \Delta x>0 \\ 0 & \text { else }\end{cases}
$$

with conductivity $G_{\mathrm{S}}$ and reversal potential $E_{\mathrm{S}}$. The SAC is thus only present if the cell is elongated. It acts as an additional stimulus on the membrane voltage, so it takes over the role of $I_{\text {stim }}(t)$ in equation 2.11. For large enough $\Delta x$ this stimulus then can cause a depolarization and thus accounts for a way of implementing MEF into the model. This description is of course oversimplified in the sense that in principle $I_{\mathrm{S}}$ could become arbitrarily large for large deformations. Apart from the fact that a real CM certainly would not survive a too large stretch, also the number of stretch-activated ion channels in the cell membrane is finite. So even if the cell could withhold any deformation, $I_{\mathrm{S}}$ would still saturate for large $\Delta x$. However, there is evidence that the linear relation between $I_{\mathrm{S}}$ and $\Delta x$ holds even for relatively large deformations (e.g. up to $20 \%$ strain amplitude in [95]). With this in mind and for the sake of simplicity, I use this simple model for MEF in the remainder of this thesis. Equations 2.11 and 2.12 for the electrical activity, 2.13 and 2.17 describing the contraction and relaxation, together with equation 2.18 for the SAC thus give a complete qualitative description of the electromechanical dynamics of a single cardiomyocyte with excitation-contraction coupling and mechanoelectric feedback.

\subsubsection{Linear viscoelastic coupling}

The previous sections 2.1.1 to 2.1.3 treated the dynamics of a single cardiomyocyte. In order to study the possible synchronization dynamics of several coupled cells, this has to be completed by a description of the mechanical coupling provided by the extracellular matrix. Following the work by Sebastian Stein $[23,32]$ and for the sake of simplicity, I model the ECM as a linear Maxwell fluid with elasticity $k_{\mathrm{c}}$ and fluidity $\gamma_{c}$. This is again not a fully realistic description because an ECM made up mainly of collagen clearly shows a nonlinear stress-strain relation [96, 97]. This nonlinearity is further studied in the experiments described in chapter 4 and [1] where it is already present at physiologically relevant strains. Still for a first qualitative approach I limit myself to the linear viscoelastic Maxwell model. For a more comprehensive explanation on linear and nonlinear rheological concepts, see section 2.3.

The spring-dashpot realization of a Maxwell fluid is sketched in figure 2.3a. It consists of a serial combination of a spring with stiffness $k_{\mathrm{c}}$ and a dashpot with damping constant $\gamma_{\mathrm{c}}$ (see e.g. [98]). There are two equivalent descriptions of the force $F_{\mathrm{c}}$ generated by the Maxwell element at elongation $\Delta x$. The first is a single ODE for

\footnotetext{
${ }^{2}$ Note that in comparison to section 3.1 the sign of $I_{\mathrm{S}}$ is changed to match with the positive sign of $I_{\text {stim }}$ in equation 2.11 .
} 


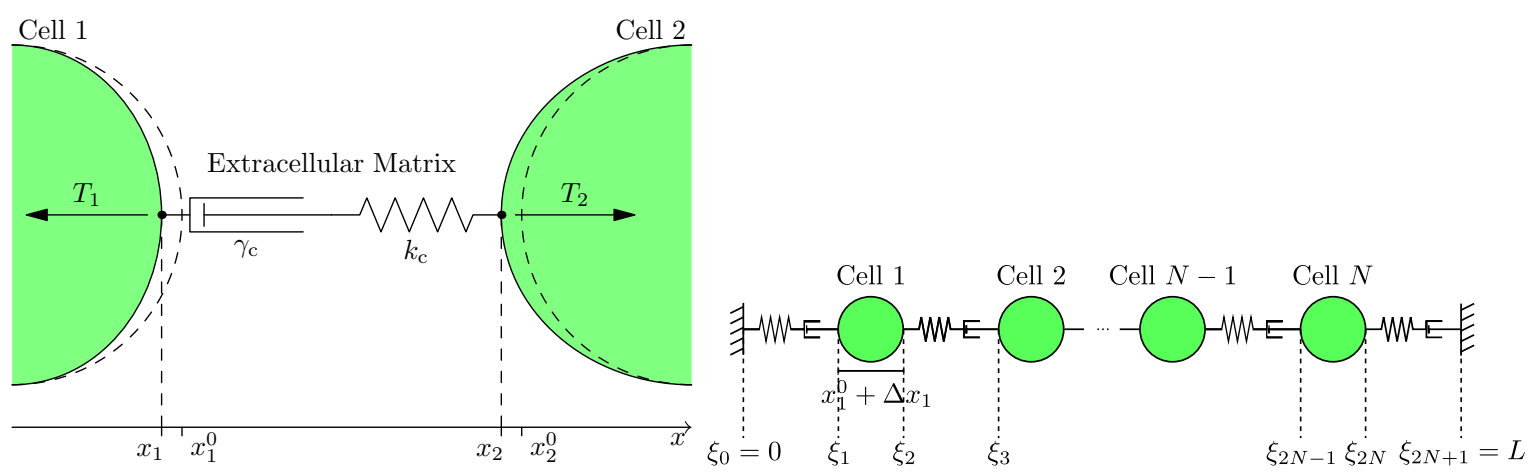

(a) Two CM coupled by a Maxwell element. (b) Linear chain of CM with fixed boundary conditions.

Figure 2.3 Sketches of viscoelastically coupled cardiomyocytes. (a) Two cells are coupled by a linear Maxwell element with fluidity $\gamma_{\mathrm{c}}$ and elasticity $k_{\mathrm{c}}$ representing the ECM. The system can be described by the motion of two points representing the position of the cell membranes of the two cells. Cell 1 is contracted $\left(\Delta x_{1}<0\right)$, cell 2 is stretched $\left(\Delta x_{2}>0\right)$. (b) A linear chain of $N$ cells coupled by Maxwell elements. Additional Maxwell elements couple the system to the fixed walls at both ends of the chain. Both sketches are taken (and in the case of (b) adapted) from section 3.1 or [33] with permission. Copyright by the American Physical Society.

the time dependency of $F_{\mathrm{c}}$

$$
\frac{\mathrm{d} F_{\mathrm{c}}}{\mathrm{d} t}=-\gamma_{\mathrm{c}} F_{\mathrm{c}}+k_{\mathrm{c}} \frac{\mathrm{d} \Delta x}{\mathrm{~d} t}
$$

the other introduces the viscous creep $d$ such that

$$
\begin{aligned}
F_{c} & =k_{c}(\Delta x-d) & \text { and } \\
\frac{\mathrm{d} d}{\mathrm{~d} t} & =\gamma_{\mathrm{c}}(\Delta x-d) . &
\end{aligned}
$$

Differentiating equation 2.20 with respect to time and inserting equation 2.21 yields equation 2.19 again, so the two formulations are indeed equivalent. The former illustrates the fluidlike behavior in that the force does not explicitly depend on the deformation, only on its time derivative. The latter illustrates how deformations are absorbed by the dashpot into the viscous creep that effectively acts as a shift of the resting position of the spring. In the following, I will use the second formulation with the viscous creep.

\section{Two coupled cells}

I now have together all the ingredients to formulate the system of two CM coupled mechanically by a linear viscoelastic ECM represented by a Maxwell element. This system is sketched in figure 2.3a. As in section 2.1.2, the motion of the cell membranes relative to each other are reduced to the motion of two point masses with virtual 
mass $m$ at locations $x_{1}$ and $x_{2}$. With respective resting positions $x_{1}^{0}$ and $x_{2}^{0}$, the cell elongations can be formulated as $\Delta x_{1}=x_{1}-x_{1}^{0}$ and $\Delta x_{2}=x_{2}^{0}-x_{2}$. Note the different signs in the definitions of $\Delta x_{1}$ and $\Delta x_{2}$ that guarantee that both cell elongations are negative when the cells are contracted. The elongation of the coupling spring is then

$$
x_{2}-x_{1}-x_{2}^{0}+x_{1}^{0}=-\left(\Delta x_{1}+\Delta x_{2}\right)
$$

leading to a coupling force $F_{\mathrm{c}}$ by the Maxwell element from equation 2.20

$$
F_{\mathrm{c}}=-k_{\mathrm{c}}\left(\Delta x_{1}+\Delta x_{2}+d\right)
$$

with viscous creep $d$ from equation 2.21 reading

$$
\frac{\mathrm{d} d}{\mathrm{~d} t}=-\gamma_{\mathrm{c}}\left(\Delta x_{1}+\Delta x_{2}+d\right)
$$

here. Including this coupling force, the EOM for both cell elongations can finally be formulated as

$$
m \frac{\mathrm{d}^{2} \Delta x_{i}}{\mathrm{~d} t^{2}}=-\gamma_{\mathrm{r}} \frac{\mathrm{d} \Delta x_{i}}{\mathrm{~d} t}-k_{\mathrm{r}} \Delta x_{i}-T_{i}-\frac{1}{2} k_{\mathrm{c}}\left(\Delta x_{1}+\Delta x_{2}+d\right)
$$

or, as above, assuming overdamping

$$
\gamma_{\mathrm{r}} \frac{\mathrm{d} \Delta x_{i}}{\mathrm{~d} t}=-k_{\mathrm{r}} \Delta x_{i}-T_{i}-\frac{1}{2} k_{\mathrm{c}}\left(\Delta x_{1}+\Delta x_{2}+d\right)
$$

$i \in\{1,2\}$. This leads to the system of two coupled cells being fully described by a set of in total nine ODEs; each cell has membrane voltage $v_{i}$ and gating $h_{i}$ from equations 2.11 and 2.12 with $\mathrm{SAC} I_{\mathrm{S}, i}$ from equation 2.18 , active contractile force $T_{i}$ from equation 2.13 and cell elongation $\Delta x_{i}$ as in equation 2.26. The ninth ODE is the viscous creep $d$ of the coupling Maxwell element in equation 2.24.

\section{Linear chain}

The reduction to the motion of just two point masses is not valid anymore in a linear chain of $N>2$ coupled cells connected by $N-1$ Maxwell elements as sketched in figure 2.3b. Now each cell is represented by two point masses at its left and right boundaries. To mimic the setting of cells in an ECM contained in a finite volume, the two outermost cells are connected to fixed walls by another two Maxwell elements. For simplicity, I assume all Maxwell elements to have the same fluidity $\gamma_{\mathrm{c}}$ and elasticity $k_{\mathrm{c}}$, and all cells to have the same parameters $k_{\mathrm{r}}, \gamma_{\mathrm{r}}$ of the passive restoration. Let $x_{i}^{0}$ be the resting length of cell $i$ and $l_{j}^{0}$ the initial resting position of Maxwell element $j$. Then

$$
L:=\sum_{i=1}^{N} x_{i}^{0}+\sum_{j=1}^{N+1} l_{j}^{0}
$$


is the constant total length of the chain. The two mass points denoting the boundaries of cell $i$ are located at $\xi_{2 i-1}$ and $\xi_{2 i}$, while $\xi_{0}=0$ and $\xi_{2 N+1}=L$ denote starting and ending point of the chain, respectively. Then the cell elongation is

$$
\Delta x_{i}=\xi_{2 i}-\xi_{2 i-1}-x_{i}^{0}
$$

which can be used to calculate the SAC $I_{\mathrm{S}, i}$ with equation 2.18 and the passive restoring force $F_{\mathrm{r}, i}$ from equation 2.14. The $j$-th Maxwell element in turn is bounded by the two neighboring cell membranes at $\xi_{2 j-2}$ and $\xi_{2 j-1}$. Its viscous creep $d_{j}$ now fulfills

$$
\frac{\mathrm{d} d_{j}}{\mathrm{~d} t}=\gamma_{c}\left(\xi_{2 j-1}-\xi_{2 j-2}-l_{j}^{0}-d_{j}\right)
$$

resulting in the force

$$
F_{\mathrm{c}, j}=k_{\mathrm{c}}\left(\xi_{2 j-1}-\xi_{2 j-2}-l_{j}^{0}-d_{j}\right) .
$$

Each cell again has an active contractile component $T_{i}$ from equation 2.13. With these forces, the EOM for each point mass $\alpha$ can be formulated as

$$
m \frac{\mathrm{d}^{2} \xi_{\alpha}}{\mathrm{d} t^{2}}=\left\{\begin{array}{ll}
\frac{1}{2}\left(F_{\mathrm{r}, \alpha / 2}-T_{\alpha / 2}\right)+\frac{1}{2} F_{\mathrm{c}, \alpha / 2+1} & \text { for } \alpha \text { even } \\
-\frac{1}{2}\left(F_{\mathrm{r},(\alpha+1) / 2}-T_{(\alpha+1) / 2}\right)-\frac{1}{2} F_{\mathrm{c},(\alpha+1) / 2} & \text { for } \alpha \text { odd }
\end{array} .\right.
$$

In this description, overdamping can not trivially be assumed since the above set of equations can not be solved explicitly for $\frac{\mathrm{d} \xi_{\alpha}}{\mathrm{d} t}$ when $m \frac{\mathrm{d}^{2} \xi_{\alpha}}{\mathrm{d} t^{2}} \rightarrow 0$. It can still be achieved by choosing $\gamma_{\mathrm{r}}$ large compared to $m$ or by using the elastostatic approach from, e.g., Weise et al. [90]. From the positions of the mass points, cell elongations and SAC can be calculated with which the ODEs for the electrical dynamics of each cell can be solved.

\subsection{Implementation and analysis}

Numerical integration of the equations of the systems of two and a linear chain of viscoelastically coupled cardiomyocytes as introduced in the previous sections is relatively straight-forward since in the end, a system of coupled ODEs has to be integrated for each of the systems for a simulation period $T_{\text {sim. }}$. For all numerical calculations and analyses, self-written Python [99] and Cython [100] codes have been used. ODE integration in the case of the two-cell system was performed using the lsoda solver with adaptive time-stepping from the SciPy Python package [101]. In all other cases, using an explicit Euler method with sufficiently small integration time step $^{3}$ proved adequate. When analyzing the time series resulting from ODE integration, usually an initial transient time $T_{\text {trans }}$ is disregarded - except, of course, when the transient itself is studied.

\footnotetext{
${ }^{3}$ See also method section in [33] and section 3.1 for the integration parameters used there.
} 
When analyzing the results of the numerical integrations to evaluate the synchronization dynamics of the coupled cells, two tasks require some attention. That is why I present them in the following in more detail. The first is a way to automatically evaluate the mode of synchronization of two viscoelastically coupled cells. The method itself makes use of the results obtained in section 3.1, so it is helpful for understanding this method to first read that section and the publication [33] therein. The second is the way phases are assigned to the cells in a linear chain and how these phases are used to evaluate the level of synchrony. For more details on how to determine the natural frequencies of individual cells and how to find initial phases and the corresponding initial conditions, see appendix A.

\subsubsection{Evaluating modes of synchronization of two oscillators}

The method to evaluate the mode of synchronization (if there is any) presented in the following is specifically devised for the case of two viscoelastically coupled excitable oscillators as presented in section 2.1.4. It requires some prior knowledge about possible resulting dynamics, e.g., by integrating the ODEs for a small set of parameters to generate time series as shown in figures 5 to 8 in section 3.1 and in [33]. Two illustrative examples are shown in figure 2.4. The method is then used to automatically evaluate the mode of synchronization of such time series, e.g., in order to generate the phase diagram in figure 9 in section 3.1. In general, I require the two coupled cells, cell 1 and cell 2 , to have different natural frequencies $\nu_{1}>\nu_{2}$ and to be identical in all other respects, in particular regarding excitability, active contraction, and MEF. The dynamics and modes of synchronization then depend on the parameters of the coupling, namely fluidity $\gamma_{\mathrm{c}}$ and elasticity $k_{\mathrm{c}}$.

By preliminary simulations for varying coupling parameters it is known that there are essentially three types of possible dynamics: asynchrony (figure 5 in section 3.1 ) whereby both cells are not influenced by each other, $n: m$ synchronization (figure $2.4 \mathrm{a}$ and figures 6 and 7 in section 3.1) including $n=m=1$, i.e., full in-phase synchronization, and a spring-dominated antiphase synchrony (figure 2.4b). It is important to observe that only in the last case the electrical dynamics of the faster cell (cell 1) is influenced by the coupling; in any other case it is unperturbed after possible initial transients. In general, the mechanical influence on one cell's membrane voltage $v_{i}$ is visible in the corresponding SAC $I_{\mathrm{S}, i}$. Lastly, I observe that in the antiphase case, the oscillations are much faster than in all other cases (see figure $2.4 \mathrm{~b}$ ).

In all cases, synchronization is evaluated after integrating the system for a simulation period of $T_{\text {sim. }}$. I obtain a nine-dimensional time series for all dynamical variables explained in section 2.1.4. I consider only times $t>T_{\text {trans }}$ to exclude transients. From the time series, the frequencies $\nu_{1,2}^{v}$ of the membrane voltages are determined by recording the time between all consecutive upward crossings through $v_{1,2}=0.5$ and averaging to first get the periods of oscillations $T_{1,2}^{v}$; the frequencies then are $\nu_{1,2}^{v}=1 / T_{1,2}^{v}$. In the same way, also the frequency $\nu_{1,2}^{\mathrm{S}}$ of the SAC are determined if there are any SAC present at all; here the threshold for the crossing events depends on the parameters. For the ones used in section $3.1, I_{\mathrm{S}, i}=5 \times 10^{-5}$ proved suitable. If 


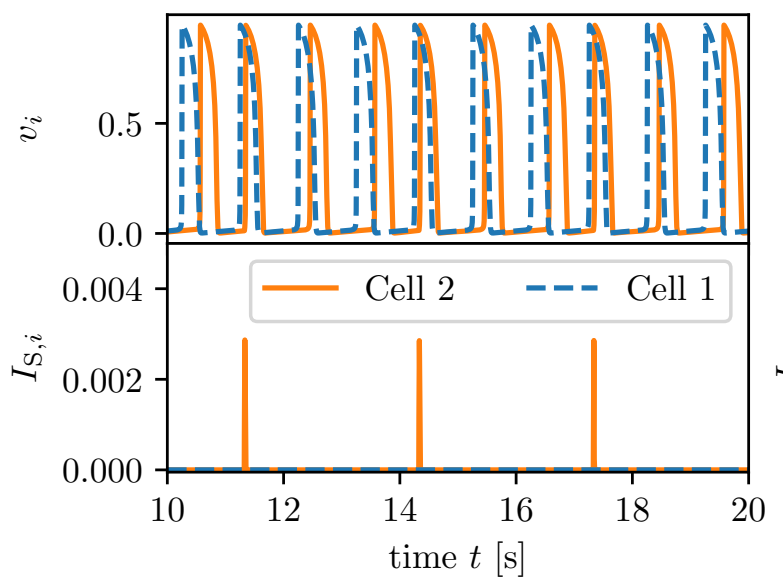

(a) 3:3 synchronization

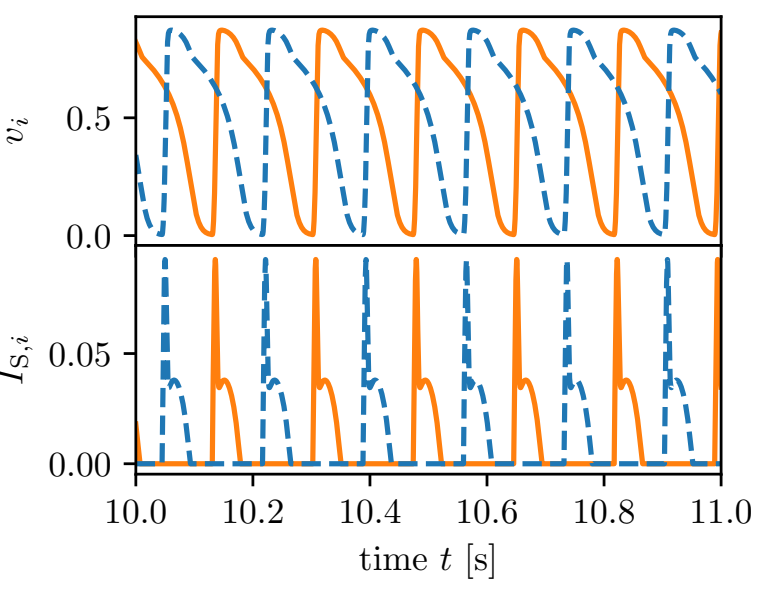

(b) antiphase synchronization

Figure 2.4 Exemplary time series of membrane voltage and SAC of two coupled cells illustrating $n: m$ synchronization (a, 3:3 shown here) and antiphase synchronization (b). In both cases, the parameters are the same as in section 3.1 and [33]. The cells have natural frequencies $\nu_{1}=1 \mathrm{~Hz}$ and $\nu_{2}=0.9 \mathrm{~Hz}$. In (a), there are nonzero SAC only in cell 2, with frequency $\nu_{2}^{\mathrm{S}}=1 / 3 \mathrm{~Hz}$. Cell 1 remains unperturbed, thus $\nu_{1}^{v}=\nu_{1}=1 \mathrm{~Hz}$ and $n=3$. The periods of the membrane voltage in cell 2 are not constant, but on average $\nu_{2}^{v}=1 \mathrm{~Hz}$, so $m=3$. In the antiphase case (b), $\nu_{i}^{v}=\nu_{j}^{\mathrm{S}}>\nu_{k}$ for any combination $i, j, k \in\{1,2\}$ (note the shorter time scale compared to (a)).

at this point there are no SAC in either of the two cells and, consequently, $\nu_{i}^{v}=\nu_{i}$, $i \in\{1,2\}$, the system is asynchronous. If on the other hand there are SAC in both of the cells and $\nu_{i}^{v}=\nu_{j}^{\mathrm{S}}>\nu_{k}$ for any combination $i, j, k \in\{1,2\}$, i.e., frequencies in both membrane voltage and SAC are larger than the natural frequencies of both cells, the dynamics is spring-dominated and synchronized in antiphase. This is shown in figure 2.4b. In principle, one still would have to check for the phase difference between the oscillations of the two cells but effectively the above conditions occur if and only if there is antiphase synchrony.

In all remaining cases I have $n: m$ synchrony; there are no SAC in cell 1 and thus, $\nu_{1}^{v}=\nu_{1} . n$ and $m$ are then determined via $\nu_{1,2}^{v}$ and $\nu_{2}^{S}: n=\nu_{1}^{v} / \nu_{2}^{\mathrm{S}}$ and $m=\nu_{2}^{v} / \nu_{2}^{\mathrm{S}}$. In particular, at full 1:1 synchrony $\nu_{1}=\nu_{1,2}^{v}=\nu_{2}^{\mathrm{S}}$ and $n=m=1$. For any $n, m$, every $m$-th beat of cell 2 , after $n$ beats of cell 1 , is triggered by a hyper-threshold SAC. The period of the whole system is then $n / \nu_{1}^{v}$ (or, equivalently, $m / \nu_{2}^{v}$ ). Figure 2.4 a shows a case of $n=m=3$ in which every third beat in cell 2 is triggered by cell 1 .

\subsubsection{Determining phases and measures of synchronization of a linear chain}

In a chain of $N$ coupled cells, possibly each of which with its own natural frequency, a simpler method of evaluating the overall synchrony in the system is needed. As introduced in section 1.5 it is convenient in these situations to reduce the dynamics of 
each individual oscillator to a single phase $\psi_{i} \in[-\pi, \pi)$. For excitable oscillators or excitable systems in general, such a phase can be assigned in various ways including delay embedding and Hilbert transforms [102-104], however, in the cases presented in section 3.1 it proved most convenient to use a linear interpolation between to consecutive maxima in the time series of the membrane voltage $v_{i}$ of the cell to determine $\psi_{i}$. Consider a time series of $v_{i}$ with several consecutive AP. At time $t$, let $t_{\text {prev }} \leq t$ be the point in time of the maximum of the previous, and $t_{\text {next }}>t$ that of the following AP. The phase can then be defined as

$$
\psi_{i}(t)=-\pi+2 \pi \frac{t-t_{\mathrm{prev}}}{t_{\mathrm{next}}-t_{\mathrm{prev}}} .
$$

Of course, this concept of a phase only works for $t$ such that there is a previous and a next AP. At the very beginning of the time series, before the first AP, and at the very end, after the last one, $\psi_{i}$ is not defined.

The phases can then be used to calculate the Kuramoto order parameter [76]

$$
R=\frac{1}{N}\left|\sum_{k=1}^{N} \exp \left(i \psi_{k}\right)\right| .
$$

For a highly ordered state, i.e., all $\psi_{i}$ being close to each other, $R \approx 1$. If, on the other hand, the system is far away from synchrony, essentially all phases $\psi_{i}$ are distributed equally in the interval $[-\pi, \pi)$ and $R \approx 0$. Note that with this order parameter, only in-phase synchronization is considered ordered. If there were two populations of oscillators synchronized in antiphase, i.e., with a phase difference of $\pi$ between each other, $R$ would still vanish as it does in the completely disordered state.

\subsection{Concepts of rheology}

Rheology is the science of the flow of complex fluids. In order to understand the experiments investigating the mechanical behavior of the extracellular matrix, I introduce the necessary rheological concepts in the following section. For a comprehensive introduction, see, e.g., [98].

Consider the situation sketched in figure 2.5. Between two plates of areas $A$ with distance $h$ to each other, a medium is located the flow of which shall be described. This medium is sheared by a force $F$ parallel to the upper plate resulting in a displacement $\Delta x$ between upper and lower plate and thus also between the upper and the lower boundary of the sample medium (assuming no slip of the medium along the plates). Since the magnitude of the displacement not only depends on the material properties of the medium but also on the geometry of the system, it is reasonable to introduce quantities instead that depend on the material properties only. For this, one defines 


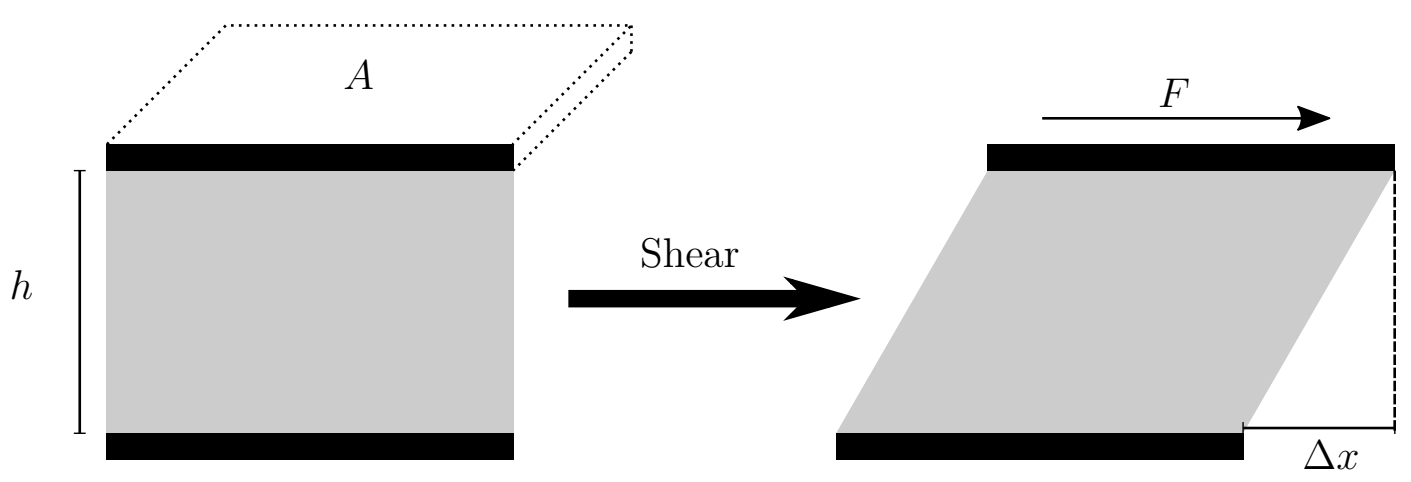

Figure 2.5 Sketch of a simple shear deformation. A medium between two plates with areas $A$ at distance $h$ is subject to a force $F$ parallel to the upper plate resulting in a displacement $\Delta x$.

the shear stress

$$
\sigma=\frac{F}{A}
$$

as the force per area that is necessary for the deformation. $\sigma$ has the unit $\mathrm{N} / \mathrm{m}^{2}=\mathrm{Pa}$. One further defines the strain

$$
\gamma=\frac{\Delta x}{h}
$$

as the ratio of displacement and the distance between the two plates. Subsequently, the strain rate or shear rate is

$$
\dot{\gamma}=\frac{\mathrm{d} \gamma}{\mathrm{d} t}=\frac{1 \mathrm{~d} \Delta x}{h} \frac{\mathrm{d} t}{.}
$$

$\gamma$ is dimensionless and is often given in $\%, \dot{\gamma}$ then has the unit $1 / \mathrm{s}$. In order to characterize the sample, either $\sigma, \gamma$, or $\dot{\gamma}$ are investigated as a function of one or both of the remaining quantities.

Figure 2.5 sketches the case of a simple shear geometry. In our experiments, we used rotational shear instead whereby a circular upper plate performs a rotational motion or rotational oscillations with respect to a fixated lower one. This case is shown in figure 2.6. Since the local deformation of the sample medium between the two plates now depends on the distance to the axis of rotation of the upper plate, strain and strain rate from equations 2.35 and 2.36 would not be uniform in the whole sample for a constant gap between the two plates [105]. This is why it is useful to use a conical upper plate with an angle of inclination $\alpha$ as in the sketch. Then, also the distance between upper and lower plate depends on the distance from the axis of rotation. The gap at the tip of the cone $h_{0}$ and $\alpha$ can now be adapted such that the variations of deformations and gap height approximately cancel out in equation 2.35 (for small gap sizes $h_{0}$ ), resulting in a uniform strain (and strain rate) everywhere in the sample. 


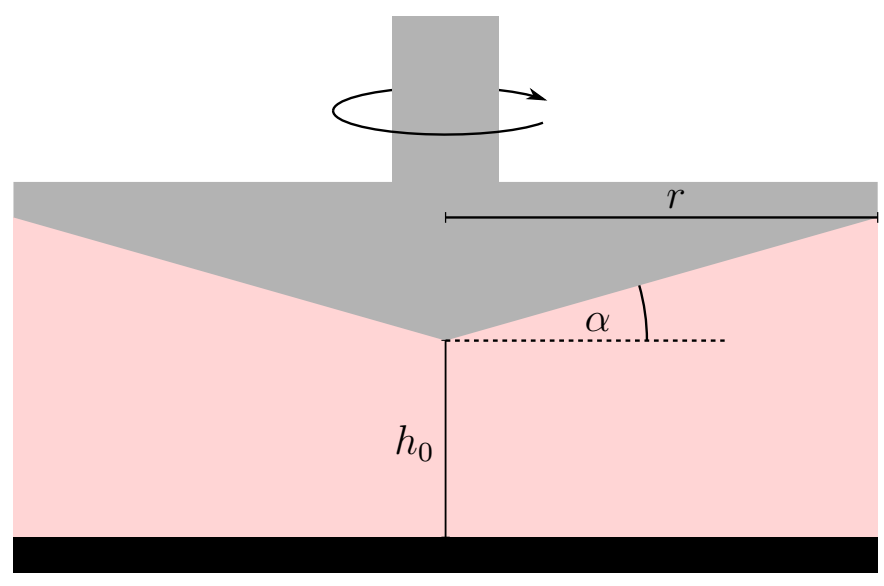

Figure 2.6 Sketch of the chamber of a rheometer with cone-and-plate geometry. The upper plate (grey) is rotating or rotationally oscillating, the lower plate (black) remains steady. Thus, the local deformation of the sample (pink) at a specific radius depends on that radius. With the conical shape of the upper plate, also the local distance between upper and lower plate depends on the radius. The gap height at the tip of the cone $h_{0}$ and the angle $\alpha$ can be adapted such that strain $\gamma$ and strain rate $\dot{\gamma}$ from equations 2.35 and 2.36 are (approximately) uniform throughout the whole sample.

\subsubsection{Linear rheology}

For infinitesimal deformations and forces, the response of a material to stress or strain input can be described by linear rheology [98]. In idealized purely elastic or purely viscous cases the stress response is proportional to strain input or strain rate of the input, respectively. With constants $E$ and $\eta$,

$$
\sigma=E \gamma
$$

for elastic and

$$
\sigma=\eta \dot{\gamma}
$$

for viscous materials. $E$ is called Young's modulus, $\eta$ is the (dynamic) viscosity. A purely elastic material can be represented by a single (ideal) spring, a purely viscous by a single dashpot. Real materials usually behave neither purely viscous nor purely elastic but viscoelastic instead. The most simple representations of viscoelastic materials are combinations of one spring and one damper. When aligned in series, the result is the Maxwell fluid introduced in section 2.1.4. Rephrasing equation 2.19 in terms of stress and strain, the Maxwell fluid is described by

$$
\eta \frac{\mathrm{d} \sigma}{\mathrm{d} t}=E(\eta \dot{\gamma}-\sigma)
$$

Equation 2.19 is recovered by reintroducing length scales from the geometrical problem to express stress and strain in terms of forces and deformations and by identifying the 
fluidity $\gamma_{\mathrm{c}}=E \eta^{-1}$. As discussed in section 2.1.4, the Maxwell fluid behaves indeed fluidlike in that the stress only depends on strain rate but not on strain itself. It therefore has no unique resting strain as is visible in the equivalent formulation with the viscous creep in equation 2.21. Aligned in parallel, a spring and a dashpot make up a Kelvin-Voigt material which is a representation of a viscoelastic solid. Its stress-strain relation is

$$
\sigma=E \gamma+\eta \dot{\gamma}
$$

which, in contrast to the Maxwell fluid, has a clear resting state $\gamma=\dot{\gamma}=0$. When there is no stress acting on a Kelvin-Voigt material it will return to this resting state.

For general materials, the linear stress response at time $t$ may depend not only on the momentary strain $\gamma(t)$ but on its history, too [98]. This can be expressed by means of a memory kernel $Q(t)$ and the integral expression

$$
\sigma(t)=\int_{0}^{t} Q\left(t-t^{\prime}\right) \gamma\left(t^{\prime}\right) \mathrm{d} t^{\prime}
$$

The stress response thus depends on the form of the strain input. For the specific case of an oscillatory strain

$$
\gamma(t)=\gamma_{0} \exp [i(\omega t+\theta)]
$$

with amplitude $\gamma_{0}$, angular frequency $\omega$, and phase $\theta$, and corresponding strain rate

$$
\dot{\gamma}(t)=i \gamma_{0} \omega \exp [i(\omega t+\theta)]=i \omega \gamma(t)
$$

the complex modulus $G^{*}$ can be defined as the steady state response to oscillatory strain. Since oscillatory strain will be used in all experiments in this thesis, the complex modulus and its real and imaginary part, $G^{\prime}$ and $G^{\prime \prime}$, are of special interest. Formally, the complex modulus is defined as

$$
G^{*}=\lim _{t \rightarrow \infty} \frac{\sigma(t)}{\gamma(t)}
$$

and may depend on $\omega$. In experimental practice, it is determined by considering the stress response averaged over (at least) one period of oscillation. When this does not change over time anymore, the complex modulus is calculated as the fraction of stress and strain. Then, with $G^{*}=G^{\prime}+i G^{\prime \prime}$ and equations 2.42 and 2.43 , the stress can be expressed [106] as

$$
\sigma=G^{*} \gamma=G^{\prime} \gamma+\frac{G^{\prime \prime}}{\omega} \dot{\gamma}
$$

Since the response is linear, this holds for any superposition of oscillatory strain inputs, including purely sinusoidal strain that is often used for convenience. The real part of 


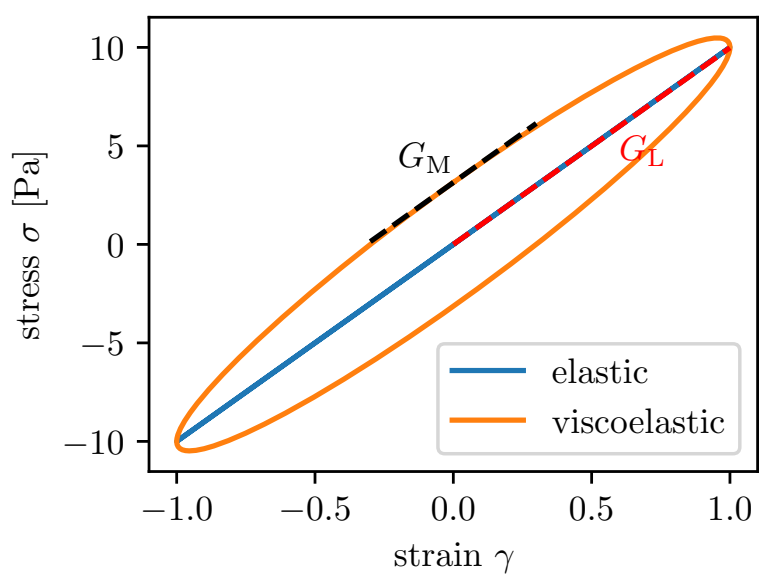

(a) Linear response

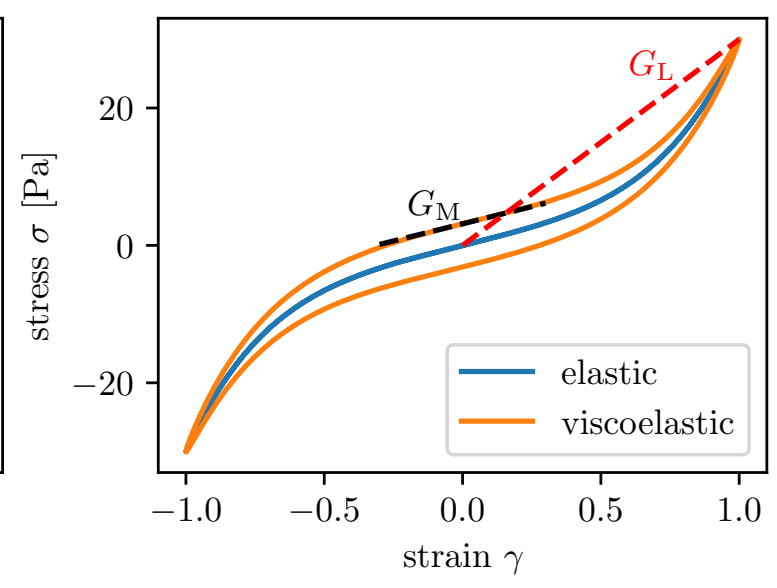

(b) Strain-stiffening

Figure 2.7 Lissajous figures in the case of linear (a) and nonlinear strain-stiffening response (b). Both, purely elastic and viscoelastic responses are shown in blue and orange, respectively. The viscoelastic stress depends on the strain rate and thus displays a hysteresis loop (depending on the sign of $\dot{\gamma}$ ). The minimal strain modulus $G_{\mathrm{M}}$ is the slope of the tangent to $\sigma$ at $\gamma=0$ indicated by a dashed black lines. Conversely, the large strain modulus $G_{\mathrm{L}}$ is the slope of the line connecting the origin with the stress at maximum strain (red dashed lines). In the linear case $G_{\mathrm{L}}=G_{\mathrm{R}}=G^{\prime}, G_{\mathrm{L}}>G_{\mathrm{M}}$ for strain-stiffening.

$G^{*}, G^{\prime}$, is called the storage modulus since it is proportional to the average portion of the deformation energy that is stored as potential energy per deformation cycle and unit volume of the material [98]. The imaginary part $G^{\prime \prime}$ is called loss modulus since it is proportional to the average dissipated energy in the same way.

\subsubsection{Nonlinear rheology and large-amplitude oscillatory shear (LAOS)}

When applying finite deformations, the stress response may depend nonlinearly on the amplitude and rate of the deformation [107]. If increasing strain leads to an increased stress response compared to the expectation from linear rheology, the response is called strain-stiffening, i.e., the material shows a stiffer-than-linear stress response to a large deformation. Conversely, when the stress response is smaller than predicted by linear theory, one speaks of strain-softening. Nonlinear dependencies on strain rate are called shear-thinning and shear-thickening [108].

As in the linear case, the stress response in general depends on the form of the strain input. In the following, I concentrate again on the special case of oscillatory strain input, since it is the one most relevant to this thesis. When the amplitude of the strain oscillation is large enough to observe nonlinear behavior, one speaks of large-amplitude oscillatory shear (LAOS) [106]. To visualize the relation between stress and strain, it is useful to plot $\sigma$ versus $\gamma$ as shown in figure 2.7 for a linear and nonlinear case. This visualization is called a Lissajous figure. Figure 2.7a sketches the stress-strain 
relation in the linear case. In blue, the purely elastic stress response from equation 2.37 is shown $-\sigma$ does not depend on $\dot{\gamma}$ and the relation of $\sigma$ and $\gamma$ is characterized by a straight line the slope of which corresponds to $E$ which in this case coincides with the storage modulus $G^{\prime}$. This line is split up into an ellipse the width of which is determined by the loss modulus $G^{\prime \prime}$ as indicated in equation 2.45. Figure 2.7b shows an example of strain-stiffening. With increasing $\gamma, \sigma$ grows stronger than linear. This feature can be illustrated by introducing the minimal strain modulus $G_{\mathrm{M}}$ and the large strain modulus $G_{\mathrm{L}}$ [106] sketched in black and red, respectively. $G_{\mathrm{M}}$ is the slope of the tangent to $\sigma(\gamma)$ at $\gamma=0$ while $G_{\mathrm{L}}$ corresponds to the slope of the line connecting the origin and the point $\left(\gamma_{0}, \sigma\left(\gamma_{0}\right)\right)$. In the linear case both are equal and correspond to $G^{\prime}$. For strain-stiffening $G_{\mathrm{L}}$ is larger than $G_{\mathrm{M}}$ while for strain-softening $G_{\mathrm{L}}<G_{\mathrm{M}}$.

Finding a mathematical description of nonlinear stress-strain relations in general is not a trivial task. In the case of periodic oscillatory strain when one can assume the steady state stress response to be periodic as well, a description via Fourier rheology can be used [106]. For simplicity I assume a sinusoidal strain

$$
\gamma(t)=\gamma_{0} \sin (\omega t)
$$

The stress response $\sigma$ can be formulated as a Fourier series

$$
\sigma\left(t, \omega, \gamma_{0}\right)=\gamma_{0} \sum_{n \text { odd }}\left[G_{n}^{\prime}\left(\omega, \gamma_{0}\right) \sin (n \omega t)+G_{n}^{\prime \prime}\left(\omega, \gamma_{0}\right) \cos (n \omega t)\right]
$$

with coefficients $G_{n}^{\prime}$ and $G_{n}^{\prime \prime}$ that may depend on strain amplitude and angular frequency. The Fourier series only includes odd modes because of symmetry assumptions to the stress response: when no strain is applied, no stress should be present, hence there is no constant term. If the direction of deformation is reverted so is the direction of the resulting force, thus even terms in the series are omitted. Using sine and cosine theorems and identifying $\dot{\gamma}(t)=\dot{\gamma}_{0} \cos (\omega t)$ where $\dot{\gamma}_{0}=\omega \gamma_{0}$, one can reformulate [106] equation 2.47 as

$$
\sigma=\gamma_{0} \sum_{n \text { odd }}\left[(-1)^{\frac{n-1}{2}} G_{n}^{\prime} T_{n}\left(\frac{\gamma}{\gamma_{0}}\right)\right]+\dot{\gamma}_{0} \sum_{n \text { odd }}\left[\frac{G_{n}^{\prime \prime}}{\omega} T_{n}\left(\frac{\dot{\gamma}}{\dot{\gamma}_{0}}\right)\right]
$$

with Chebyshev polynomials $T_{n}(x)$ [109]. For $n=1$ this corresponds to the linear case in equation 2.45. From equation 2.48 it becomes clear that the stress $\sigma$, as in the linear case in equation 2.45 can be split into two parts depending only on strain or strain rate, respectively. The former is called elastic, the latter viscous stress. The elastic stress is shown in blue in figure 2.7. From equation 2.48, $G_{\mathrm{L}}$ and $G_{\mathrm{M}}$ can be calculated directly: $G_{\mathrm{M}}$ is found by differentiating and inserting $\gamma=0$,

$$
G_{\mathrm{M}}=\left.\frac{\mathrm{d} \sigma}{\mathrm{d} \gamma}\right|_{\gamma=0}=\sum_{n \text { odd }} n G_{n}^{\prime}
$$


whereas $G_{\mathrm{L}}$ is the ratio of stress at maximum strain and strain amplitude,

$$
G_{\mathrm{L}}=\frac{\sigma\left(\gamma=\gamma_{0}\right)}{\gamma_{0}}=\sum_{n \text { odd }} G_{n}^{\prime}(-1)^{\frac{n-1}{2}}
$$

From $G_{\mathrm{M}}$ and $G_{\mathrm{L}}$ the strain-stiffening ratio $S$ used in [1] is calculated as

$$
S=\frac{G_{\mathrm{L}}-G_{\mathrm{M}}}{G_{\mathrm{L}}} .
$$

In the linear regime $S=0$, for strain-stiffening $S>0$, and $S<0$ for strain-softening.

The framework of Fourier rheology is universally applicable to the setting of oscillatory shear but the resulting stress-strain relation depends on the amplitude and the frequency of the oscillations via the coefficients $G_{n}^{\prime}(\omega, t)$ and $G_{n}^{\prime \prime}(\omega, t)$. Other approaches to model nonlinear stress-strain relations for individual or classes of materials include fractional rheology where non-integer derivatives of $\sigma, \gamma$, and $\dot{\gamma}$ are considered (e.g. [110]). Constitutive laws can be formulated for Neo-Hookean and Mooney-Rivin materials expressing weak nonlinearities in their stress responses [111]. Specifically for cardiac tissue, the Holzapfel-Ogden model has been introduced [112].

\subsection{Experimental methods and analysis}

After having introduced the general language of rheology in the previous section, I will now explain the experimental setup and protocols used to measure the rheological properties of the ECM consisting of collagen with and without cells. The original aim of the experiments was to establish a mathematical model for the (nonlinear) stress-strain relation of the ECM which then can be used to replace the simplistic linear Maxwell model in the numerical simulations of coupled CM as introduced in section 2.1.4. In the course of these measurements, the influence of the presence and absence of $\mathrm{CM}$ and fibroblasts on the development of the ECM itself became an interesting question of its own.

All rheological experiments were conducted in the lab of Florian Rehfeldt, Third Institute of Physics - Biophysics, University of Göttingen, who helped devising the protocols. Most of the experiments until April 2018 were performed together with Susanne Schlick, Institute for Pharmacology and Toxicology, UMG, who also provided all cells used in the experiments.

\subsubsection{The rheometer}

The rheological experiments are conducted on an Anton Paar MCR501 rotational shear rheometer that is depicted in figure 2.8a. The sample is placed in between a fixed lower plate and an upper probe or measuring system (see figure 2.8b) whose rotational motion imprints either a stress or a strain on the sample. The respective resulting strain or stress response is recorded. The measuring system can be exchanged in order 


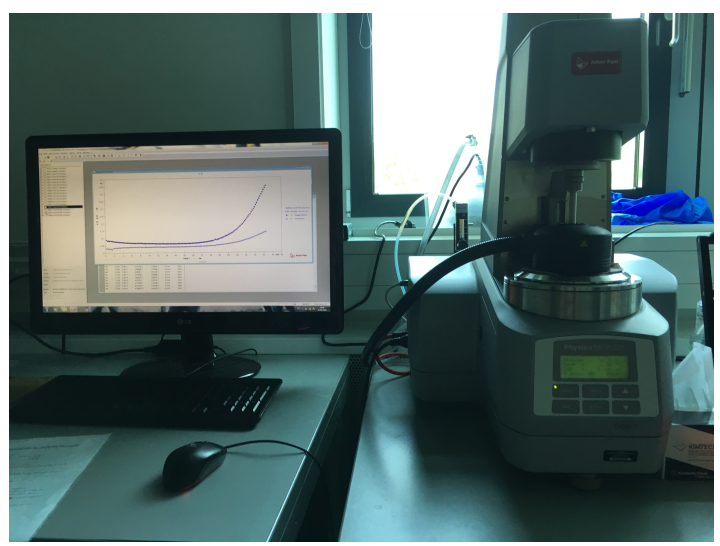

(a) Photograph of the rheometer with PC running the measurement software.

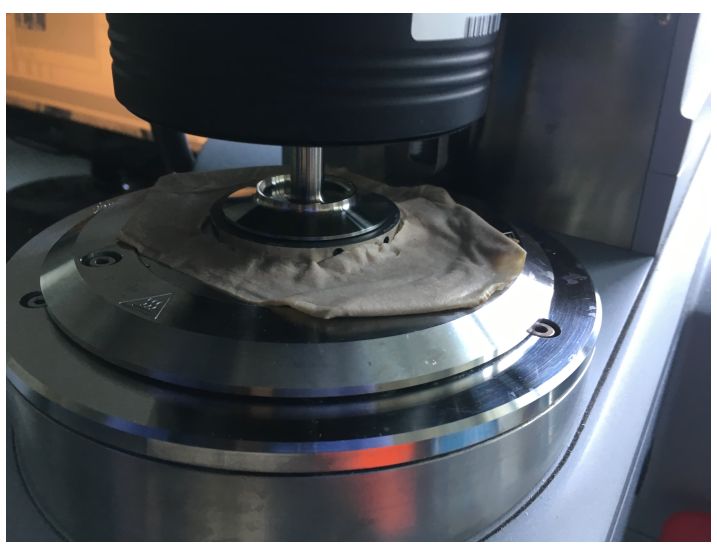

(b) Close-up of the chamber of the rheometer.

Figure 2.8 Setup of the rheological experiments. (a) The measurements are conducted with an Anton Paar MCR 501 rheometer that is controlled with the manufacturer's proprietary RheoPlus or RheoCompass software. (b) The sample is placed in the climate chamber between the lower plate (chromium shown) and the measuring system with conical shape (CP50-1 shown here). Drying out of the sample is prevented by placing moistened tissues around the plates. During a measurement, the chamber is closed as in (a).

to allow for different radii, angles and geometries. The sample is located inside a climate chamber (black in figure 2.8b) that keeps it at a fixed temperature. To further prevent drying out of the samples, moistened tissues are placed inside the chamber but far enough from the plates as to not touch plates or sample and thus distort the measurement.

In all measurements presented in this thesis, a cone-and-plate geometry (see section 2.3 and figure 2.6) was used, i.e., the measuring system had a conical shape to allow for a single unique strain $\gamma$ in the entire sample medium [105]. Cones with different radii and angles resulting in different sample volumes have been used for the experiments in this thesis; all are listed in table 2.1.

For the lower plate, both chromium oxide and steel were tested to see whether the material and thus the exact boundary conditions had an influence on the measurements. As we found no difference, most experiments were performed using the chromium oxide

Table 2.1 The different measuring systems or probes used in the rheological experiments. All measuring systems have conical shape and consist of steel. From the radii, gap heights and angles $\left(r, h_{0}\right.$, and $\alpha$ in figure 2.6, respectively) of the measuring systems follows the corresponding sample volume.

\begin{tabular}{|l|c|c|c|c|}
\hline Probe & Radius $[\mathrm{mm}]$ & Gap height $[\mu \mathrm{m}]$ & Angle $\left[^{\circ}\right]$ & Sample volume $[\mu \mathrm{l}]$ \\
\hline CP25-1 & 12.5 & 48 & 1 & 80 \\
CP50-1 & 25 & 101 & 1 & 570 \\
CP25-2 & 12.5 & 103 & 2 & 140 \\
\hline
\end{tabular}


lower plate. All measuring systems consist of steel. Of these, mainly CP25-2 and CP50-1 were used.

The measurements are controlled using the manufacturer's proprietary software. Over the course of the experiments two versions were used: Until September 2018 and again from June 2019, the older RheoPlus software was used, from September to December 2018, Anton Paar provided us with a trial version of the more recent RheoCompass software that allows for user-defined strain input $\gamma(t)$ (see section 2.4.3). Since the new software could not resolve existing issues with nonlinear measurements (see section 4.2), I switched back to the old one after the end of the trial period.

\subsubsection{Collagen hydrogels with and without cells}

The experiments performed for this thesis can be divided into two groups. The first group consists of all experiments until April 2018 and includes measurements on collagen hydrogels with and without cardiomyocytes, human foreskin fibroblasts (HFF), or both. When both cell types are present, the mixture corresponds exactly to the EHM recipe used by the Zimmermann group [57]. This recipe by Susanne Schlick is listed in table 2.2 for the two batches of bovine collagen type I that were used with it. The recipe is also explained in the methods section of [1]. It consists of collagen dissolved in acetic acid and $0.1 \mathrm{~N} \mathrm{NaOH}$ for neutralization, in addition to $2 \mathrm{x}$ RPMI medium (Roswell Park Memorial Institute medium) and serum free mammalian cell culture medium (SFMM) that also contains the cells if there are any. The final hydrogel has a collagen concentration of $0.92 \mathrm{mg} / \mathrm{ml}$, in line with the $0.8-1.2 \mathrm{mg} / \mathrm{ml}$ used for EHM production in [57]. More details on the constituents of EHM can be found in [14, 15, 57]. All cells were provided by Susanne Schlick and the group of Wolfram-Hubertus Zimmermann, Institute of Pharmacology and Toxicology, UMG.

From the constituents, the hydrogels are produced with the volumes in table 2.2 as follows. First the constituents (stored at $5{ }^{\circ} \mathrm{C}$ ) are cooled on ice. Still on ice, dissolved collagen and 2x RPMI are mixed in an $1500 \mu \mathrm{l}$ Eppendorf tube. $0.1 \mathrm{~N} \mathrm{NaOH}$ is added for neutralization and to trigger the onset of gelation of the collagen. RPMI contained phenol red to make this neutralization visible. At last SFMM is added - depending on the protocol, with or without cells. Even without cells, an equal amount of SFMM

Table 2.2 Recipes for the two batches of collagen used with the EHM protocol as established by S. Schlick, UMG. The SFMM medium may or may not contain cells depending on the experimental protocol. The resulting $1000 \mu$ hydrogels contain a collagen concentration of $0.92 \mathrm{mg} / \mathrm{ml}$ and. If cells are present, they are added to the SFMM in a concentration of $950000 \mathrm{ml}^{-1}$ for HFF only, $1900000 \mathrm{ml}^{-1}$ for CM only, or $2850000 \mathrm{ml}^{-1}$ in $1: 2$ ratio for both HFF and CM.

\begin{tabular}{|c|c|c|c|c|c|}
\hline Batch & $\begin{array}{c}\text { Concentration } \\
{[\mathrm{mg} / \mathrm{ml}]}\end{array}$ & $\begin{array}{c}\text { Collagen } \\
{[\mu \mathrm{l}]}\end{array}$ & $\begin{array}{c}\text { 2xRPMI } \\
{[\mu \mathrm{l}]}\end{array}$ & $\begin{array}{c}\text { 0.1N NaOH } \\
{[\mu \mathrm{l}]}\end{array}$ & $\begin{array}{c}\text { SFMM } \\
{[\mu \mathrm{l}]}\end{array}$ \\
\hline 15CSA02 & 6.1 & 152.5 & 152.5 & 28.6 & 716 \\
17CSA03 & 6.49 & 142 & 142 & 26.5 & 739 \\
\hline
\end{tabular}


is used to make the final gels with and without cells as comparable as possible. The whole hydrogel is mixed and a volume depending on the rheometer probe (see table 2.1) is placed onto the lower plate of the rheometer chamber. Since the hydrogels are very viscous, reverse pipetting [113] is used in this last step for maximal accuracy in the sample volume. In the rheometer, the gel is heated to $23^{\circ} \mathrm{C}$ and the rheological measurement (cf. section 2.4.3) is performed. This protocol for producing the hydrogels was used for all results presented in [1].

From June 2018 on, I continued with measurements of collagen hydrogels without cells, both for generating data to model its nonlinear rheology and for comparing the properties of different batches of collagen. To facilitate the latter the protocol for hydrogel preparation was changed. The two media necessary for cells, SFMM and RPMI, were replaced by PBS (Phosphate Buffered Saline). This allowed for a better control of the $\mathrm{pH}$ of the final hydrogel which is crucial when comparing the gelation properties of different collagens. Since the gelation process is highly sensitive to changes in $\mathrm{pH}$ [70], this has to be as comparable as possible among all gels. Similar $\mathrm{pH}$ proved to be easier to achieve with the PBS protocol instead of the EHM protocol. Of course, the protocol could only be changed since no cells were used in any of the gels. To determine the volumes needed for the hydrogels, I used the EHM protocol from [15] by Malte Tiburcy, Institute for Pharmacology and Toxicology, UMG, who provided the collagen batches as a basis. For $1000 \mu \mathrm{l}$ of hydrogel with PBS, I used the same amount of collagen as for the same volume of EHM, resulting in a collagen concentration of $0.88 \mathrm{mg} / \mathrm{ml}$. Note that as the EHM protocol was refined from [57] to [15], the collagen concentration in the hydrogels is decreased compared to the above protocol. Then I measured the $\mathrm{pH}$ of this mixture using a $\mathrm{pH}$ meter and added $0.1 \mathrm{~N} \mathrm{NaOH}$ until a $\mathrm{pH}$ of approximately 7.7 was reached. The final amounts determined that way are listed in table 2.3.

Preparing the PBS hydrogels for rheometry follows that line: As before the constituents are cooled on ice from their storage temperature of $5{ }^{\circ} \mathrm{C}$. Collagen and $1 \mathrm{x}$ PBS are mixed, $0.1 \mathrm{~N} \mathrm{NaOH}$ is added and the sample volume determined by the measuring system in use is placed in the rheometer where the gel is heated to $23^{\circ} \mathrm{C}$ and the measurement is performed.

Results obtained with the two different protocols are of course not entirely comparable quantitatively, although the general features of the gelation and the linear and nonlinear rheological properties remain the same (see chapter 4).

\subsubsection{Experimental protocols}

After having placed the hydrogel, prepared with either of the two protocols, inside the rheometer the actual measurement is started. In almost all protocols - except for the prestress measurements (see below) - an oscillatory shear $\gamma(t)$ is exerted on the sample by the rotating measuring system and the stress response $\sigma(t)$ is measured. In most 
Table 2.3 Recipes for $1000 \mathrm{pl}$ collagen hydrogels created with the PBS protocol for collagen comparison measurements without cells. The amount of $0.1 \mathrm{~N} \mathrm{NaOH}$ added was determined such that the resulting hydrogel has a $\mathrm{pH}$ of $\approx 7.7$. This has to be determined for each batch separately. Following an updated mixture protocol by M. Tiburcy, UMG, the final collagen concentration in the hydrogel is $0.88 \mathrm{mg} / \mathrm{ml}$, so slightly lowered compared to table 2.2 .

\begin{tabular}{|c|c|c|c|c|}
\hline Batch & $\begin{array}{c}\text { Concentration } \\
{[\mathrm{mg} / \mathrm{ml}]}\end{array}$ & $\begin{array}{c}\text { Collagen } \\
{[\mu \mathrm{l}]}\end{array}$ & $\begin{array}{c}\text { 1xPBS } \\
{[\mu \mathrm{l}]}\end{array}$ & $\begin{array}{c}0.1 \mathrm{~N} \mathrm{NaOH} \\
{[\mu \mathrm{l}]}\end{array}$ \\
\hline 14CSA02 & 6.56 & 134.1 & 865.9 & 23 \\
15CSA02 & 6.4 & 137.5 & 862.5 & 23 \\
17CSA03 & 6.49 & 135.6 & 864.4 & 17 \\
18CB023001 & 6.49 & 135.6 & 864.4 & 25 \\
17CB024002 & 6.72 & 131 & 869 & 21 \\
17CB024001 & 6.91 & 127.4 & 872.6 & 23 \\
18CB024001 & 6.81 & 129.2 & 870.8 & 15 \\
18CB024003 & 6.84 & 128.7 & 871.3 & 17 \\
\hline
\end{tabular}

cases, this strain has sinusoidal form

$$
\gamma(t)=\gamma_{0} \sin (2 \pi f t)
$$

with strain amplitude $\gamma_{0}$ and frequency $f$. In special cases, however, other forms of $\gamma(t)$ - waveforms - are used. The strain amplitude $\gamma_{0}$ is used to control whether the linear or nonlinear regime of the sample is probed (see section 2.3). Small $\gamma_{0}$ corresponds to a linear stress response. At sufficiently large values of $\gamma_{0}$, the polymerized hydrogel will show a nonlinear response [96].

\section{Time sweeps}

In all experiments the gelation process of the hydrogel is observed during a time sweep of duration $T_{\mathrm{TS}}$. This is then possibly followed by further measurements. During a time sweep, storage modulus $G^{\prime}$ and loss modulus $G^{\prime \prime}$ are recorded in intervals $\Delta t=15 \mathrm{~s}$ by averaging the stress response to a sinusoidal strain over each of these time intervals (cf. equation 2.45, automatically done by the rheometer software). At the same time, temperature and normal force $F_{\mathrm{N}}$ exerted by the sample on the probe are recorded. Since $G^{\prime}$ and $G^{\prime \prime}$ are inherently linear quantities, a small strain amplitude $\gamma_{0}=1 \%$ is chosen to safely remain in the linear regime. The small strain amplitude ${ }^{4}$ ensures that the gelation process is not influenced by the measurement itself. A frequency $f=1 \mathrm{~Hz}$ is chosen for the time sweeps because it is close to the actual beating frequency of the CM in EHM [23] and thus physiologically relevant and also because measurements at frequencies close to $1 \mathrm{~Hz}$ proved most reliable (see section 4.2).

\footnotetext{
${ }^{4}$ Mind that even for the largest cone CP50-1, $\gamma_{0}=1 \%$ corresponds to a maximal deformation of about $5 \mu \mathrm{m}$ at the very edges of the sample.
} 

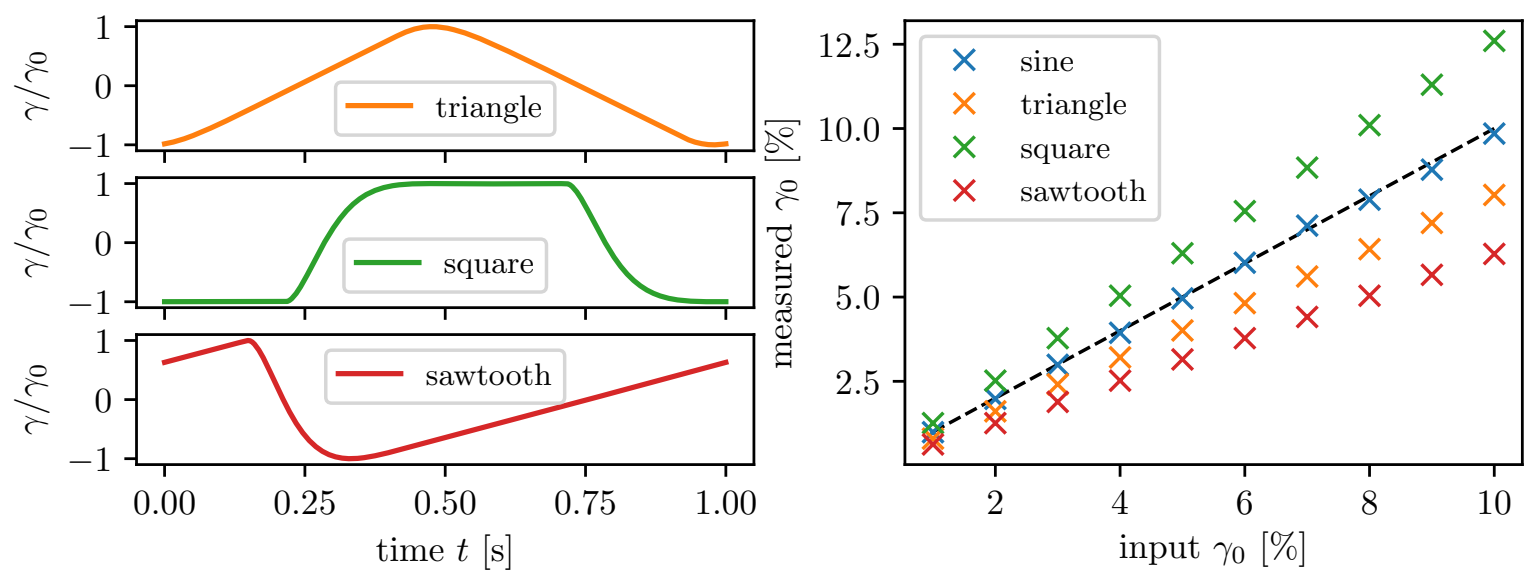

Figure 2.9 Nonsinusoidal waveforms. The left panels show the normalized strain input at a frequency of $1 \mathrm{~Hz}$ for triangle, square, and sawtooth strain input. The right panel shows the deviation of the strain amplitudes $\gamma_{0}$ that were actually measured from those that were set. The identity is indicated with a black dashed line. While measured and set $\gamma_{0}$ agree for sinusoidal strain, they deviate strongly for the nonsinusoidal waveforms.

\section{Large-amplitude oscillatory shear (LAOS)}

A time sweep is usually followed by a LAOS (large-amplitude oscillatory shear) measurement in which the time series $\sigma(t)$ of the stress response to the strain $\gamma(t)$ is recorded directly. In sinusoidal LAOS measurements, $\gamma(t)$ still has the sinusoidal form from equation 2.52. Now $\gamma_{0}$ is step-wise increased to observe the nonlinear regime of the sample. A nonlinear response becomes directly visible in the stress response $\sigma(t)$ (see section 2.3.2). $\sigma(t)$ is recorded over one period of $\gamma(t)$, then the strain amplitude is increased. Each period is sampled with 256 data points. The range of strain amplitudes usually was between $1 \%$ and $10 \%$, in rare cases it was increased up to $\gamma_{0}=20 \%$. The frequency can of the strain input can also be varied; I used $0.1 \mathrm{~Hz} \leq f \leq 10 \mathrm{~Hz}$ to probe the frequency dependence of the response as well. The measurements with complete recording of the time series $\sigma(t), \gamma(t)$, and $\dot{\gamma}(t)$ are called "waveform measurements" in the Anton Paar software. Note that for these measurements, one control loop regulating the position control - called dynamic position control - of the rheometer has to be switched off. The strain is thus likely to be less precise in LAOS measurements compared to the ones without waveform recordings. If or how much less however is hard to quantify since the raw data for $\sigma, \gamma$, and $\dot{\gamma}$ can only be obtained when this control feature is switched off in the first place; a comparison with data obtained with dynamic position control thus is impossible.

This LAOS measurements can be conducted in the same way but with different waveforms, i.e., strain input. Both controlling softwares have predefined triangle, square, and sawtooth profiles that can be applied instead of the sine. The resulting normalized strain inputs as recorded in such a waveform measurement with nonsinusoidal strain are shown in the left panels of figure 2.9. It is clearly visible that the ideally instantaneous changes in square and sawtooth are softened as well as the maximum of the triangle 


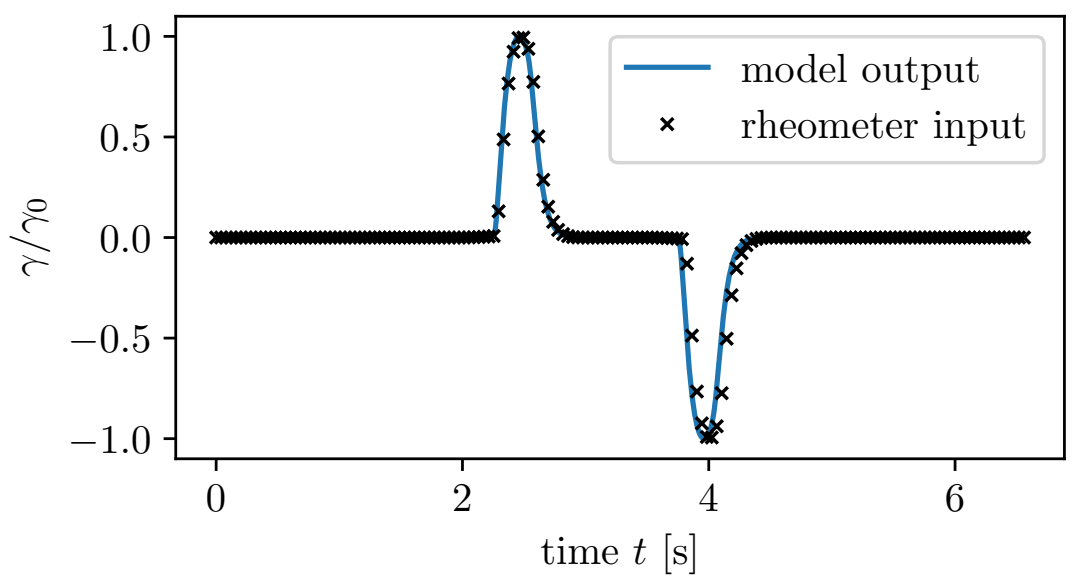

Figure 2.10 Normalized contraction data obtained by integrating the ECC model from section 2.1.2 for a single cell as shown in figure 2.2b. The contraction curve is mirrored, before, in between and after the contraction curves relaxation times are filled in (blue curve). The curve is sampled with maximal $30 \mathrm{~Hz}$ and the resulting data points are used as a user-defined strain input for the rheometer.

waveform. One problem that arose during these measurements is shown in the right panel of figure 2.9 - the actual applied strain amplitude deviated from the one set in the control software as an input for the nonsinusoidal strains. While it was consistently too large in the case of square strain, it was significantly decreased for triangle and sawtooth input, with relative deviations being as high as $20 \%$ for triangle and square and over $35 \%$ in case of sawtooth. This deviations have to be kept in mind when choosing the range of applied strain amplitude for these waveforms since they may by accident lead to different applied strains compared to the sine LAOS measurements. Too small amplitudes may result in not reaching the nonlinear regime while a too large amplitude may cause the sample to slip along the surface of one of the plates of the rheometer. In the latter case the measured strain does not (solely) correspond to a deformation of the sample and the data cannot be used anymore.

In order to test mathematical models for the stress response of the ECM, I used strain input created from the contraction curves resulting from the electromechanical CM model introduced in section 2.1 for a single cell. For this user-defined strain input, the RheoCompass software has to be used. The input from the contraction curves is depicted in figure 2.10. To generate this input, the contraction curve from figure $2.2 \mathrm{~b}$ is used. First it is normalized and extended by relaxation periods of $2 \mathrm{~s}$ with $\gamma=0$. Additionally, the contraction curve is mirrored in order to have symmetrical deflection of the probe of the rheometer. In between the two excursions, an additional relaxation period of length $0.5 \mathrm{~s}$ is inserted. This curve is then sampled to create individual data points for the strain input - here, a sampling rate larger than $30 \mathrm{~Hz}$ produced errors with the measurement software, so $30 \mathrm{~Hz}$ is used. As before, the stress response $\sigma(t)$ is recorded. The normalized, sampled data then are multiplied with the desired strain amplitude. In between two successive strain data points, the strain is interpolated 
linearly.

\section{Prestrain and prestress}

An alternative to LAOS measurements for probing the nonlinear rheology of the ECM are prestrain measurements, whereby an initial strain is applied that is then used as the base level for the oscillatory strain. The general concept is to probe possible nonlinearities by applying different prestrain amplitudes; the oscillation amplitude itself then can be small enough to only cause a linear response - the strength of which varies depending on the prestrain. Unfortunately, the drive of the rheometer does not allow for simultaneous application of prestrain and oscillatory strain. Therefore I performed prestress measurements instead. The general idea is the same - on top of an initial stress $\sigma_{\text {pre }}$ an oscillatory stress with amplitude $\sigma_{0}$ and frequency $f$ is applied resulting in a stress

$$
\sigma(t)=\sigma_{\text {pre }}+\sigma_{0} \sin (2 \pi f t)
$$

the resulting strain and strain rate $\gamma(t)$ and $\dot{\gamma}(t)$ are measured. As with the prestrain measurements, the nonlinearity is probed by increasing $\sigma_{\text {pre }}$. The oscillation amplitude $\sigma_{0}$ remains sufficiently small such that the response to the oscillation remains linear. For the gels tested with this protocol I used $0.1 \mathrm{~Pa} \leq \sigma_{\text {pre }} \leq 1.3 \mathrm{~Pa}$ and $\sigma_{0}=0.05 \mathrm{~Pa}$. The frequency of oscillation is varied between $1 \mathrm{~Hz}$ and $4 \mathrm{~Hz}$.

One issue with this type of measurements was the rheometer not being able to simultaneously record the prestrain resulting from the application of a prestress and the additional strain stemming from the oscillatory stress. Instead it would only record the strain resulting from the oscillatory stress around an (unknown) origin from the prestress. I tried to compensate for this shortcoming by measuring the strain resulting from a constant stress $\sigma_{\text {pre }}$ without an oscillation on top of it before the oscillatory measurement. Because of a possible viscous creep of the sample resulting effectively in a shift of base strain level, this calibration is limited in precision.

To reduce the influence of a previous applied $\sigma_{\text {pre }}$, between all measurements with different $\sigma_{\text {pre }}$ and $f$, a relaxation period of $300 \mathrm{~s}$ is applied during which strain and stress are recorded to ensure that the medium indeed relaxes. As above it is possible to either record $\sigma(t), \gamma(t)$, and $\dot{\gamma}(t)$ directly via waveform recording or averaged linear quantities like storage and loss modulus; the latter with the possible advantage of all control features being active. Since the original idea for the prestress measurements was to circumvent issues occurring with all LAOS measurements at frequencies other than $1 \mathrm{~Hz}$ (cf. section 4.2), having the full position control active seemed desirable. Furthermore, $\sigma_{0}$ is chosen such that the strain response to the oscillation is linear. Compared to the averaged quantities, the full time series do not contain new information (cf. section 2.3.2). Nevertheless, the full waveform recordings can be used as a test if the response is linear, so I performed both types of measurements. 



\section{Chapter 3}

\section{Synchronization of viscoelastically coupled excitable oscillators}

\subsection{Dynamics of two coupled cells and of linear chains}

The following section consists of the article

F. Spreckelsen, S. Luther, and U. Parlitz. "Synchronization of viscoelastically coupled excitable oscillators". In: Physical Review E 100.3 (Sept. 2019), p. 032214. DOI: 10.1103/PhysRevE.100.032214

reprinted with permission from [33]. Copyright (2019) by the American Physical Society.

The study builds on the paper published by Stein et al. [32] and on the work in Sebastian Stein's PhD thesis [23]. Modelling the viscoelastic coupling via the ECM as linear Maxwell element was taken from Sebastian's work. I combined this coupling and the existing models for electrical excitation [85, 86], mechanical contraction and stretchactivated current $[91,94]$ to a description of CM as excitable oscillators. Ulrich and I devised the data analysis. The Python code for the simulations and the data analysis was written by me, as were the figures in the manuscript. All authors contributed to the writing of the manuscript. Further details to the model and the implementation can be found in sections 2.1 and 2.2, and in appendix A. 


\title{
Synchronization of viscoelastically coupled excitable oscillators
}

\author{
Florian Spreckelsen $\odot{ }^{1,2,3, *}$ Stefan Luther, ${ }^{1,2,3,4, \dagger}$ and Ulrich Parlitz ${ }^{1,2,3, 末}$ \\ ${ }^{1}$ Max Planck Institute for Dynamics and Self-Organization, Am Faßberg 17, D-37077 Göttingen, Germany \\ ${ }^{2}$ University of Göttingen, Institute for the Dynamics of Complex Systems, Friedrich-Hund-Platz, 1, D-37077 Göttingen, Germany \\ ${ }^{3}$ DZHK (German Center for Cardiovascular Research), Partner Site Göttingen, Robert-Koch-Straße 42a, D-37075 Göttingen, Germany \\ ${ }^{4}$ University Medical Center Göttingen (UMG), Institute of Pharmacology and Toxicology, \\ Robert-Koch-Straße 40, D-37075 Göttingen, Germany
}

(Received 4 July 2019; published 23 September 2019)

\begin{abstract}
Viscoelastically coupled excitable oscillators are used to model individually beating spatially separated cardiomyocytes surrounded by an extracellular matrix (ECM). We investigate how mechanical coupling via the ECM can synchronize two such oscillators with excitation contraction coupling and electromechanical feedback and how this synchronization depends on the rheological properties of the ECM. Extending our study to a linear chain of coupled oscillators we find a transition to synchronization as the ECM becomes stiffer. In the case of purely elastic coupling we observe antiphase chimera states.
\end{abstract}

DOI: 10.1103/PhysRevE.100.032214

\section{INTRODUCTION}

The heart as a muscle is composed of individual heart muscle cells called cardiomyocytes whose coordinated contraction enables the pumping of the whole organ. The mechanical contraction of each cell is triggered by an electrical excitation in the membrane voltage following a process known as excitation-contraction coupling [1]. Each excitation is followed by a refractory period in which the cell cannot be excited again.

In recent years, the engineering of cardiac muscle tissue (engineered heart muscle) has become a perspective of treating heart failure in the future [2]. Engineered heart muscle is derived from a mixture of premature cardiomyocytes and nonmyocytes and a scaffold-gel-like collagen [3]. Initially, the cardiomyocytes beat individually. During the evolution to functioning engineered tissue that exhibits coordinated and directed contraction, the individual cardiomyocytes synchronize their beating [4]. It is hypothesized that this synchronization occurs due to their mechanical coupling via the extracellular matrix, i.e., the surrounding gel stiffened by the nonmyocytes [5]. The cells deform their surrounding by contracting, thus indirectly stretching their neighboring cells. If the deformation is sufficiently large, the cells' mechanoelectric feedback leads to a subsequent electrical excitation, most probably via the opening of so called stretch-activated ion channels [6,7]. Thus, the electrical activity of a cardiomyocyte can be influenced by a mechanical stimulus, providing a possible pathway for synchronization by purely mechanical coupling [8].

In a more general picture the oscillating cells embedded in the extracellular matrix can be seen as viscoelastically coupled excitable oscillators. Building on previous

\footnotetext{
*florian.spreckelsen@ds.mpg.de

†stefan.luther@ds.mpg.de

‡ulrich.parlitz@ds.mpg.de
}

work on viscoelastic coupling of self-sustained oscillators [9], we consider oscillators with excitation dynamics, excitationcontraction coupling, and mechanoelectric feedback coupled by linear Maxwell elements, i.e., the serial combination of a harmonic spring and a dashpot. We begin by investigating the possible dynamics of a system of two such cells and then extend our study to linear chains of different lengths. Although motivated by the system of cardiomyocytes embedded in a viscoelastic matrix we also investigate the special case of purely elastic coupling.

\section{MATHEMATICAL MODEL}

Each cell or oscillator is mainly described by the electrical excitation, i.e., its membrane voltage, and its elongation. The latter is influenced by the former via an active contractile force representing the excitation-contraction coupling while the mechanoelectric feedback represented by a stretch-activated current describes how the elongation in turn influences the excitation. As in [9], viscoelastic coupling via the extracellular matrix is modelled with a linear Maxwell element, a serial combination of a harmonic spring and a dashpot.

\section{A. Excitation dynamics}

Although there are numerous models for the dynamics of the electrical excitation of cardiomyocytes of almost arbitrary levels of abstraction and complexity, we limit ourselves to a modified Mitchell-Schaeffer model [10] as presented by Djabella et al. [11]. It is a model with two dynamical variables: the normalized membrane voltage $v$ and a generalized gating variable $h$. The ordinary differential equations (ODE) for $v$ and $h$ are

$$
\begin{gathered}
\frac{d v}{d t}=\frac{h(v+a)^{2}(1-v)}{\tau_{\text {in }}}-\frac{v}{\tau_{\text {out }}}, \\
\frac{d h}{d t}=\epsilon(v)\left[h_{\infty}(v)-h\right],
\end{gathered}
$$




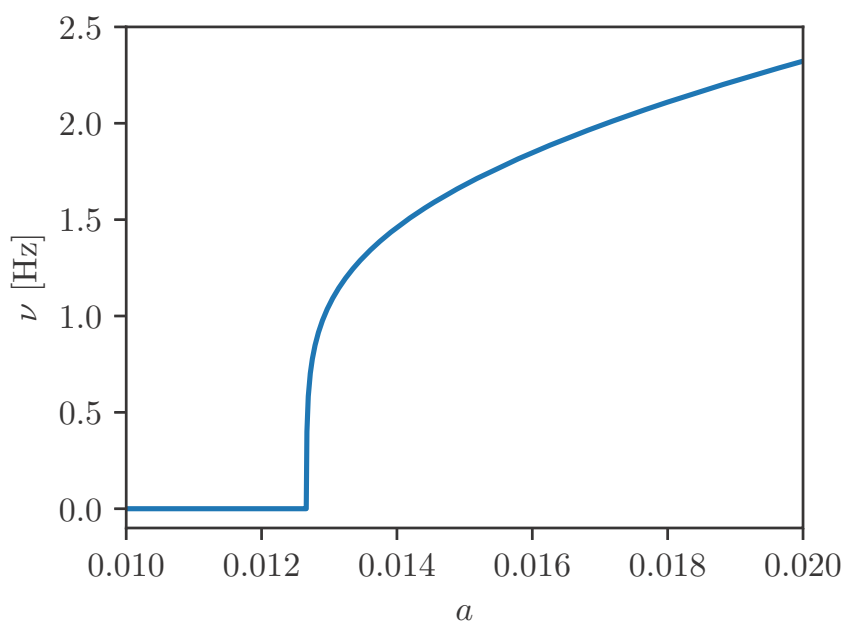

FIG. 1. The model parameter $a$ switches on self-excited oscillations of the membrane voltage $v$ and tunes the frequency $v$ of these oscillations.

where

$$
\epsilon(v)=\frac{1}{\tau_{\text {close }}}+\frac{\tau_{\text {close }}-\tau_{\text {open }}}{\tau_{\text {close }} \tau_{\text {open }}} h_{\infty}(v)
$$

and

$$
h_{\infty}(v)=\frac{1}{2}\left[1-\tanh \left(\frac{v-v_{\text {gate }}}{\eta_{\text {gate }}}\right)\right] .
$$

The different $\tau_{x}, x \in\{$ in, out, open, close $\}$, are characteristic time scales of the action potential; $v_{\text {gate }}$ and $\eta_{\text {gate }}$ are gating parameters. In the special case of $\eta_{\text {gate }}=0$, Eq. (4) becomes

$$
h_{\infty}(v)=\frac{1}{2}\left[1-\operatorname{sgn}\left(v-v_{\text {gate }}\right)\right],
$$

where $\operatorname{sgn}(\cdot)$ denotes the sign function. The parameter $a$ in Eq. (1) controls the onset and frequency of the oscillations of the membrane voltage of each cell as is shown in Fig. 1 for the parameters in Table I. Notably, for these parameters, the frequencies cover the physiological range of around $1 \mathrm{~Hz}$ for human cardiomyocytes [3]. In the nonoscillatory regime, i.e., $a<0.0126$, the resting state of Eqs. (1) and (2) is $v=0$ and $h=1$.

\section{B. Excitation-contraction coupling and mechanoelectric feedback}

We model the excitation-contraction cycle by using the active contractile force $T$ from Weise and Panfilov [12,13], thus neglecting the explicit role of calcium. The time evolution of $T$ is given by the ODE,

$$
\frac{d T}{d t}=\epsilon_{\mathrm{T}}\left(k_{\mathrm{T}} v-T\right)
$$

TABLE I. Parameters of the electrical excitation (same as used in [11]).

\begin{tabular}{lccccc}
\hline \hline$\tau_{\text {in }}$ & $\tau_{\text {out }}$ & $\tau_{\text {open }}$ & $\tau_{\text {close }}$ & $v_{\text {gate }}$ & $\eta_{\text {gate }}$ \\
\hline $0.3 \mathrm{~ms}$ & $6 \mathrm{~ms}$ & $120 \mathrm{~ms}$ & $150 \mathrm{~ms}$ & 0.13 & 0.001 \\
\hline \hline
\end{tabular}

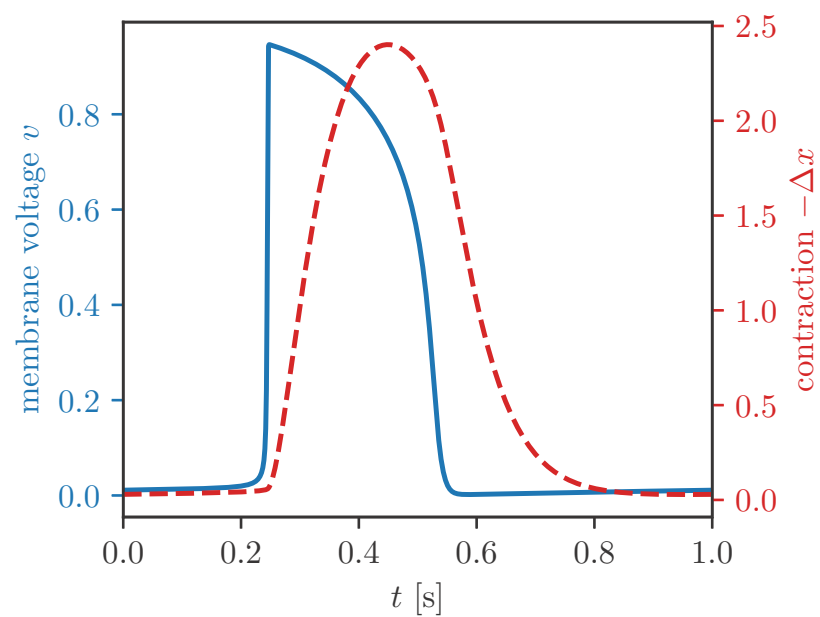

FIG. 2. An action potential (blue, continuous line) drives the active contraction (red, dashed line, arbitrary units) of a single cell.

where $\epsilon_{\mathrm{T}}$ and $k_{\mathrm{T}}$ are parameters. This differs from [12,13] in that Weise and Panfilov use a step function of $v$ for $\epsilon_{\mathrm{T}}$ that they also use in their model of the membrane voltage to account for different time scales of excitation and recovery. We found that the step function could be replaced by a constant when using the modified Mitchel-Schaeffer model for the membrane voltage instead. The active contractile force is opposed by a harmonic relaxation $F_{\mathrm{r}}=-k_{\mathrm{r}} \Delta x-\gamma_{\mathrm{r}} \frac{d \Delta x}{d t}$ with damping $\gamma_{\mathrm{r}}$ and stiffness $k_{\mathrm{r}}$. Figure 2 shows the time series of the normalized membrane voltage and the contraction in arbitrary units, i.e., the negative cell elongation $-\Delta x$, of a single cell. Keeping in mind the biological system of cardiomyocytes in a viscoelastic matrix, we assume overdamping, i.e., vanishing inertia $\frac{d^{2} \Delta x}{d t^{2}}=0$. The contraction follows the electrical excitation and relaxes back to $\Delta x=0$ after the excitation vanishes.

The mechanoelectric feedback is implemented using the stretch-activated current $I_{\mathrm{S}}$ from Weise and Panfilov, too. It depends on the cell elongation $\Delta x$ and on the membrane potential and is only present when the cell is stretched, i.e., if $\Delta x>0$.

$$
I_{\mathrm{S}}= \begin{cases}G_{\mathrm{S}} \Delta x\left(v-E_{\mathrm{S}}\right) & \text { if } \Delta x>0, \\ 0 & \text { else. }\end{cases}
$$

$G_{\mathrm{S}}$ and $E_{\mathrm{S}}$ are conductivity and reversal potential of the stretch-activated channels, respectively. When present, $I_{\mathrm{S}}$ is added as an additional stimulus on the membrane voltage, thus Eq. (1) becomes

$$
\frac{d v}{d t}=\frac{h(v+a)^{2}(1-v)}{\tau_{\text {in }}}-\frac{v}{\tau_{\text {out }}}-I_{\mathrm{S}} .
$$

The parameters for the excitation-contraction coupling, the overdamped relaxation, and the mechanoelectric feedback are listed in Table II and are mainly taken from $[12,13]$.

\section{Viscoelastic coupling}

The mechanical coupling via the extracellular matrix is modeled by a linear Maxwell element with fluidity $\gamma_{\mathrm{c}}$ and elasticity $k_{\mathrm{c}}$. As sketched in Fig. 3, the Maxwell element couples the cell membranes of two neighboring cells, each 
TABLE II. Parameters of excitation-extraction-coupling and mechanoelectric feedback as used in [12,13], together with the parameters for the (overdamped) harmonic relaxation.

\begin{tabular}{cccccc}
\hline \hline$k_{\mathrm{T}}$ & $\epsilon_{\mathrm{T}}$ & $k_{\mathrm{r}}$ & $\gamma_{\mathrm{r}}$ & $G_{\mathrm{S}}$ & $E_{\mathrm{S}}$ \\
\hline 1.5 & 0.03 & 0.5 & 30 & 2.5 & 1 \\
\hline \hline
\end{tabular}

displaying the excitation-contraction dynamics described above. The dynamics of the cell elongation is then reduced to the dynamics of motion of two mass points with (arbitrary) mass $m=1$ on which the force due to the coupling element acts as well as the contractile and restoring forces described above. The choice of the Maxwell element for the viscoelastic coupling follows the previous study by Stein et al. [9] and is mainly for reasons of simplicity. It recovers basic linear properties but of course does not show any nonlinear strainstiffening behavior.

\section{Equations of motion of two coupled cells}

The dynamics of the electrical excitation of two cells coupled as sketched in Fig. 3 follow Eqs. (8) and (2) with the stretch-activated current $I_{\mathrm{S}}$ Eq. (7). They influence each other only by the deformation of the coupling element and resulting stretch-activated currents. The points describing each cell membrane's position $x_{i}$ have resting position $x_{i}^{0}, i \in\{1,2\}$. In the case of two cells, it is convenient to define cell elongations as $\Delta x_{1}:=x_{1}-x_{1}^{0}$ and $\Delta x_{2}:=x_{2}^{0}-x_{2}$. Thus, $\Delta x_{i}>0$ for both cells if the cell is stretched and $\Delta x_{i}<0$ during contraction. The coupling force is then

$$
F_{\mathrm{c}}=-k_{\mathrm{c}}\left(\Delta x_{1}+\Delta x_{2}+d\right)
$$

with viscous creep $d$ fulfilling

$$
\frac{d d}{d t}=-\gamma_{\mathrm{c}}\left(\Delta x_{1}+\Delta x_{2}+d\right) .
$$

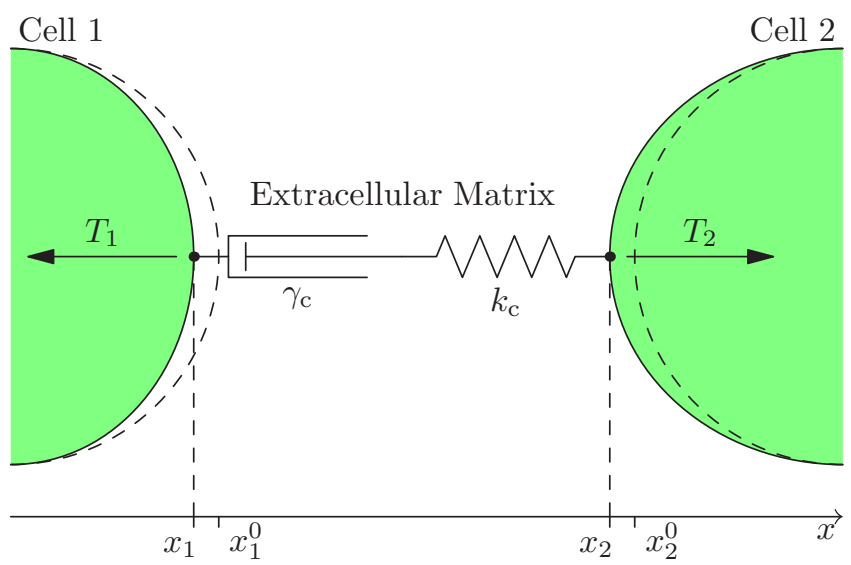

FIG. 3. Sketch of two cells coupled by a linear Maxwell element with fluidity $\gamma_{c}$ and elasticity $k_{c}$. The resting positions of the cells are indicated as dashed arcs; cell 1 contracts, cell 2 is elongated. The dynamics of the cell elongations is described by the notion of two points on which the coupling force acts as well as the active contractile and the passive restoring forces.

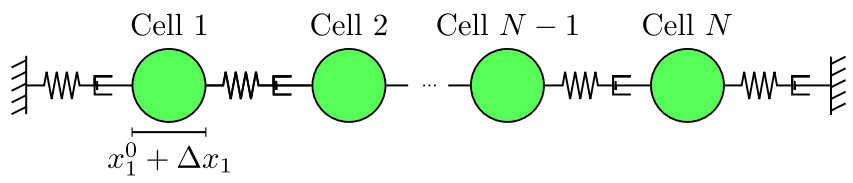

FIG. 4. Sketch of a linear chain of $N$ excitable cells coupled by $N-1$ identical Maxwell elements. To mimic the situation of cells in matrix in a confined volume, the cells at both ends of the chain, cell 1 and cell $N$, are coupled to the boundaries of the system via two additional Maxwell elements of which the outermost end points are kept fixed. Each cell has an elongation $\Delta x_{i}$ and a resting length $x_{i}^{0}$. $\Delta x_{i}$ is the result of the forces acting on the cell by the neighboring springs and its own active contraction and passive restoration (not shown).

Both cell elongations obey the equation of motion

$m \frac{d^{2} \Delta x_{i}}{d t^{2}}=-\gamma_{\mathrm{r}} \frac{d \Delta x_{i}}{d t}-k_{\mathrm{r}} \Delta x_{i}-T_{i}-\frac{1}{2} k_{\mathrm{c}}\left(\Delta x_{1}+\Delta x_{2}+d\right)$.

The mass $m$ of the mass points effectively representing the inertia of the cell membranes is (arbitrarily) set to 1. As in the case of a single cell, considering the biological system, we assume overdamped dynamics to mimic the system of coupled cardiomyocytes embedded in a viscoelastic matrix, so Eq. (11) becomes

$$
\gamma_{\mathrm{r}} \frac{d \Delta x_{i}}{d t}=-k_{\mathrm{r}} \Delta x_{i}-T_{i}-\frac{1}{2} k_{\mathrm{c}}\left(\Delta x_{1}+\Delta x_{2}+d\right),
$$

leading to a system of in total nine ODEs for both cells.

\section{E. Extension to a linear chain}

Coupling more than two cells in a linear chain requires a slightly different description than the two-cell case that essentially reduced to the motion of two mass points representing the positions of the cell membranes. This reduction is not possible when considering $N>2$ cells coupled mechanically to their direct neighbors. The cells are still represented by springs with stiffness $k_{\mathrm{r}}$, damping $\gamma_{\mathrm{r}}$, and active driving $T_{a}$, connected by $N-1$ Maxwell elements as before. To mimic the conditions of cells embedded in matrix inside a confined volume, we now add another Maxwell element to each of the two cells at the end of the chain. The end points of these outermost elements are kept fixed. Figure 4 shows a sketch of the chain with the fixed boundary conditions. A detailed overview over the equations of motion for the linear chain can be found in Appendix A.

\section{RESULTS}

All results are obtained with the parameters listed in Tables I and II corresponding to the ones used in [11-13]. The only quantities we vary are the fluidity $\gamma_{\mathrm{c}}$ and the elasticity $k_{\mathrm{c}}$ of the coupling Maxwell elements and the frequencies of the cells by tuning the corresponding parameter $a$ in Eq. (8) (cf. Fig. 1). We start by investigating the dynamics of two cells with different frequencies $v_{1}>v_{2}$ coupled by a single Maxwell element and then extend our findings to a linear 


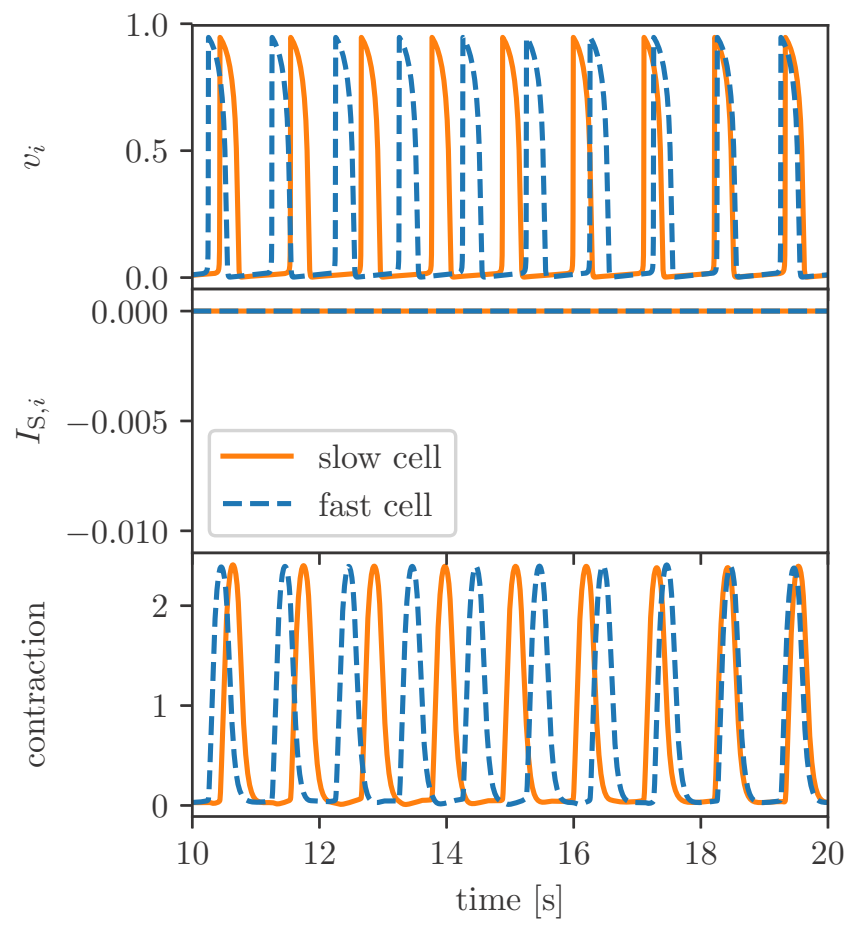

FIG. 5. Time series of membrane voltage $v_{i}$, stretch-activated currents $I_{\mathrm{S}, i}$, and contractions of two coupled cells at $k_{\mathrm{c}}=4$ and high fluidity $\gamma_{\mathrm{c}}=3$. The cells are effectively uncoupled at high fluidity; there are no stretch-activated currents in either of the two cells. Thus, both cells beat unperturbed at their natural frequencies, i.e., the fast one at $v_{1}=1 \mathrm{~Hz}$ (dashed blue lines) and the slow one at $v_{2}=0.9 \mathrm{~Hz}$ (orange lines).

chain of $N$ such cells coupled to their direct neighbors by identical Maxwell elements.

\section{A. Dynamics of two coupled oscillators}

We first turn to the system of two coupled cells 1 and 2 as sketched in Fig. 3 with natural frequencies $v_{1}>v_{2}$. In the following, we will use $v_{1}=1 \mathrm{~Hz}$ (corresponding to $a=0.01293)$ and $v_{2}=0.9 \mathrm{~Hz}(a=0.01283)$. The system of ODE introduced in Sec. II D is integrated for varying fluidity $\gamma_{\mathrm{c}}$ and elasticity $k_{\mathrm{c}}$ over an overall period of simulation of $200 \mathrm{~s}$. We initialize both cells with $v_{i}(0)=0, h_{i}(0)=1, i \in$ $\{1,2\}$ (corresponding to the resting state of a nonoscillating cell). Initially, there is no contractile force in either of the two cells and they are not elongated: $T_{i}(0)=0, \Delta x_{i}(0)=0$. There is no initial viscous creep in the coupling Maxwell element: $d(0)=0$.

The two cells are coupled in such a way that an action potential in one cell can be triggered by the other cell's contraction if it causes enough deformation of the former to induce a hyperthreshold stretch-activated current. For a given contraction strength, the parameters of the coupling Maxwell element determine whether one cell can be depolarized at all by the other's contraction. If $\gamma_{\mathrm{c}}$ is too large compared to $k_{\mathrm{c}}$, the cells are not deformed by each other's contraction; the deformation is absorbed entirely in the viscous creep of the coupling element. This case is shown in Fig. 5 which shows

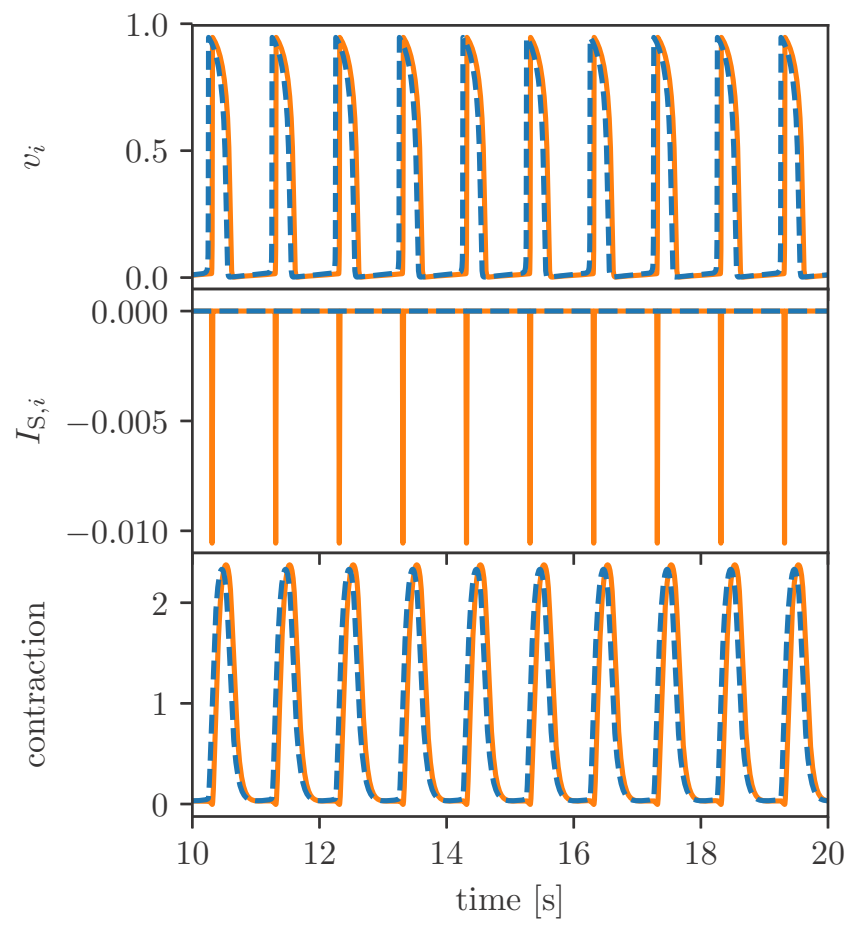

FIG. 6. Time series of membrane voltage $v_{i}$, stretch-activated currents $I_{\mathrm{S}, i}$, and contractions of two coupled cells at $k_{\mathrm{c}}=4$ and low fluidity $\gamma_{\mathrm{c}}=1$. As in the case of high fluidity in Fig. 5, the fast cell oscillates at its natural frequency $v_{1}=1 \mathrm{~Hz}$. At low fluidity however, the fast cell fully entrains the slow one: each contraction of the fast cell induces a hyperthreshold stretch-activated current in the slow cell which thus oscillates with the fast cell's frequency of $1 \mathrm{~Hz}$, too. The system is fully $(1: 1)$ synchronized; there are no stretch-activated currents in the fast cell.

the time series of membrane voltage $v_{i}$, stretch-activated currents $I_{\mathrm{S}, i}$, and contraction $-\Delta x_{i}$ of the two cells coupled at $k_{\mathrm{c}}=4$ and $\gamma_{\mathrm{c}}=3$. Both cells beat unperturbed at their natural frequencies; there are no stretch-activated currents in either of the cells.

If on the other hand $\gamma_{\mathrm{c}}$ is small enough compared to $k_{\mathrm{c}}$, the cells sense each other's contraction. The corresponding time series for two cells coupled under these conditions are shown in Fig. 6 for $k_{\mathrm{c}}=4, \gamma_{\mathrm{c}}=1$. Here, the faster cell 1 fully entrains the slower cell 2 ; each action potential in cell 2 is triggered by a stretch-activated current caused by the contraction of cell 1 . Both cells beat at $v=v_{1}=1 \mathrm{~Hz}$ and are fully $(1: 1)$ synchronized. Cell 1 is unperturbed by the beating of cell 2; there is no stretch-activated current in cell 1. Cell 2 lags behind because of the delay between excitation and contraction (cf. Fig. 2) in cell 1 on the one hand, and the finite time it takes cell 1 to deform the Maxwell element on the other hand. Thus, the exact duration of the lag increases with $\gamma_{\mathrm{c}}$ and decreases with $k_{\mathrm{c}}$.

For intermediate combinations of $k_{\mathrm{c}}$ and $\gamma_{\mathrm{c}}$ between the regimes of $1: 1$ synchronization and asynchrony, there are solutions in which the slow cell is only partially entrained by the fast one in that not each but only every $n$th contraction of the fast cell induces a hyperthreshold stretch-activated current in the slow one. In between the induced action potentials, the 


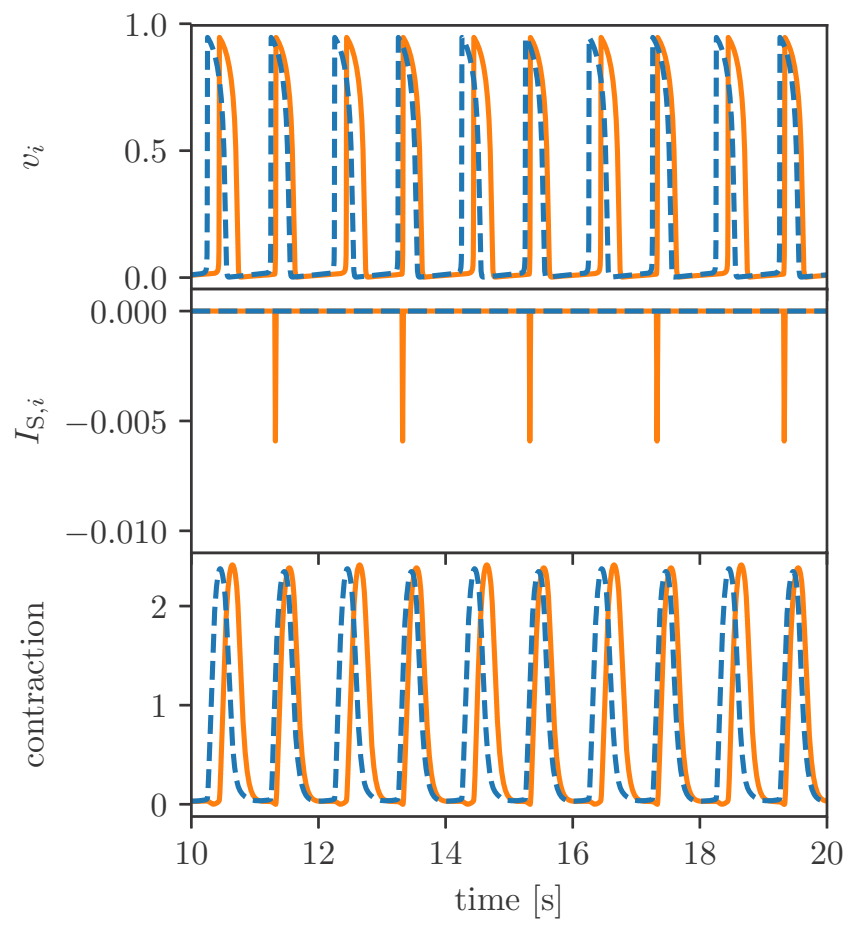

FIG. 7. Time series of membrane voltage $v_{i}$, stretch activated currents $I_{\mathrm{S}, i}$, and contractions of two coupled cells at $k_{\mathrm{c}}=4, \gamma_{\mathrm{c}}=$ 1.4. At this intermediate fluidity, only every other contraction of the fast induces a hyperthreshold stretch-activated current in the slow cell. Consequently, the time intervals between two consecutive beats of the slow cell are not constant anymore; the system shows $2: 2$ synchrony.

slow cell beats at its natural frequency, so the time intervals between two consecutive beats are not constant. Figure 7 shows one such case for $k_{\mathrm{c}}=4, \gamma_{\mathrm{c}}=1.4$ where every other beat of cell 2 is induced by the contraction of cell 1 leading to a $2: 2$ synchronization of the two cells. As in the $1: 1$ case, cell 1 is unperturbed; thus the overall period of the entire system is two times the period of the fast cell. For larger $\gamma_{\mathrm{c}}$ we also find $3: 3$ synchronization.

One special case is $\gamma_{\mathrm{c}}=0$, i.e., purely elastic coupling. Then the right hand side of Eq. (10) is 0 and the overall dimensions of the system of ODEs is reduced by 1 . If then $k_{\mathrm{c}}$ is large enough to overcome the internal damping of the cells $\left(k_{\mathrm{c}}>0.016\right.$ for our choice of parameters), the coupling spring dominates the overall dynamics. Exemplary time series of this case are shown in Fig. 8 for $k_{\mathrm{c}}=4$. Note the different time scale compared to Figs. 5-7. Both cells now oscillate in antiphase at a frequency $v \approx 6 \mathrm{~Hz}>v_{1,2}$ that is determined by the value of $k_{c}$. In both cells, each action potential is triggered by a hyperthreshold stretch-activated current. The amplitude of the action potentials and the contraction are reduced compared to the previous viscoelastic cases. In the following, we denote this behavior as $-1: 1$ synchronization.

The different dynamics of two coupled cells described above can be summarized into the phase diagram shown in Fig. 9. It shows the mode of synchronization as a function of the two Maxwell parameters fluidity $\gamma_{c} \in[0,4]$ and elasticity $k_{\mathrm{c}} \in[0,8]$. We find an asynchronous region for high $\gamma_{\mathrm{c}}$ and

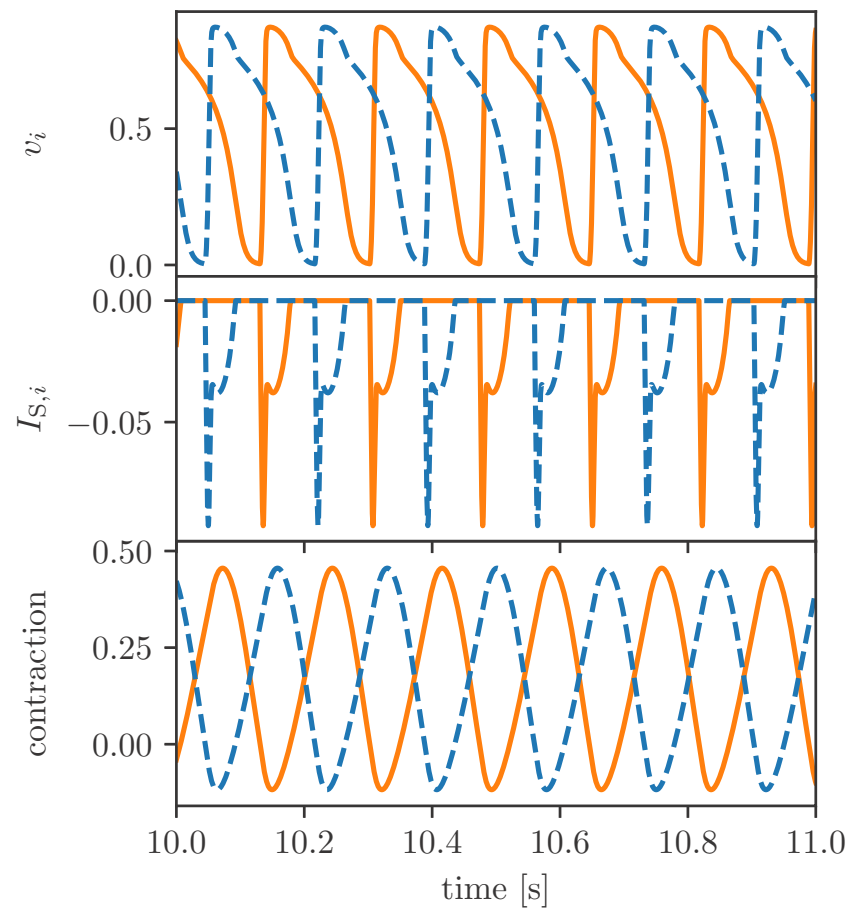

FIG. 8. The special case of purely elastic coupling $\left(k_{\mathrm{c}}=4, \gamma_{\mathrm{c}}=\right.$ 0 ) shows antiphase synchrony at a frequency $v \approx 6 \mathrm{~Hz}$ (note the shorter time scale compared to Figs. 5-7) determined by the stiffness of the coupling spring. Each action potential in both cells is triggered by a stretch-activated current. The amplitudes of action potential and contraction are reduced in both cells in comparison to the previous viscoelastic cases.

low $k_{\mathrm{c}}$ and a region of $1: 1$ synchronization for high $k_{\mathrm{c}}$ and low $\gamma_{\mathrm{c}}$. In between, there is the regime of $n: n(2: 2$ and $3: 3$ ) synchronization where the slow cell is only partially entrained. The four regions are separated by almost straight lines. These boundaries however are smeared out because of coexistence of the two neighboring solutions. This coexistence is shown in Fig. 10 that displays the mode of synchronization at constant $k_{\mathrm{c}}=4$ for consecutively increasing or decreasing $\gamma_{\mathrm{c}} \in[1,3]$ (red line in Fig. 9). As described above, we observe the spring dominated fast $-1: 1$ synchronization at $\gamma_{\mathrm{c}}=0$ for $k_{\mathrm{c}}>0.016$. This solution ceases to exist as soon as $\gamma_{\mathrm{c}}>0$.

The exact shape of the regions of different types of synchronization in the phase diagram depends on the frequencies $v_{1}$ and $v_{2}$, but the same qualitative dynamical regimes exist also for different detuning: There is an asynchronous region at high $\gamma_{\mathrm{c}}$, low $k_{\mathrm{c}}$ and a $1: 1$ region at high $k_{\mathrm{c}}$, low $\gamma_{\mathrm{c}}>0$ separated by one or several different $n: n$ or $n: m$ regions. For $\gamma_{\mathrm{c}}=0$ and sufficiently large $k_{\mathrm{c}}$ we find $-1: 1$ synchrony.

\section{B. Linear chain}

We now continue by extending the system of two viscoelastic cells to a linear chain of $N$ oscillating cells coupled only to their direct neighbors by identical Maxwell elements with fluidity $\gamma_{\mathrm{c}}$ and elasticity $k_{\mathrm{c}}$ as sketched in Fig. 4 . The cells are identical except for their frequencies $\nu_{i}$ and corresponding $a_{i}$; all other parameters remain as listed in Tables I and II as 


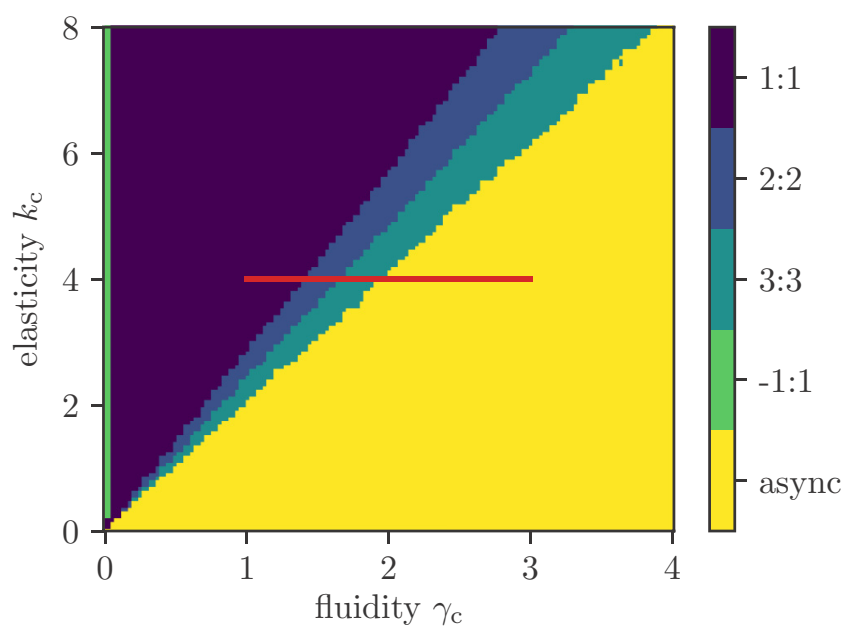

FIG. 9. The phase diagram of the mode of synchronization as function of fluidity $\gamma_{\mathrm{c}}$ and elasticity $k_{\mathrm{c}}$ of the coupling Maxwell element. For large $\gamma_{\mathrm{c}}$, low $k_{\mathrm{c}}$, the cells beat asynchronously and are essentially decoupled (yellow region). Vice versa, at low $\gamma_{c}$, high $k_{\mathrm{c}}$, the cells are fully $1: 1$ synchronized and beat at the frequency of the faster cell. In between there are regions of $n: n(2: 2,3$ : 3 ) synchronization. For the purely elastic case $\gamma_{c}=0$, we observe antiphasic $-1: 1$ synchrony for $k>0.016$.

before. The frequencies are randomly assigned from a normal distribution around $1 \mathrm{~Hz}$ with a standard deviation of $0.05 \mathrm{~Hz}$. For easier analysis we define a phase $\psi_{i}(t) \in[-\pi, \pi)$ for each of the oscillators by interpolating linearly between the points in time of two consecutive maxima of the membrane voltage $v_{i}$. The phases are used to calculate the Kuramoto order parameter [14],

$$
R=\frac{1}{N}\left|\sum_{k=1}^{N} \exp \left(i \psi_{k}\right)\right|,
$$

to evaluate the synchronization of the overall system.

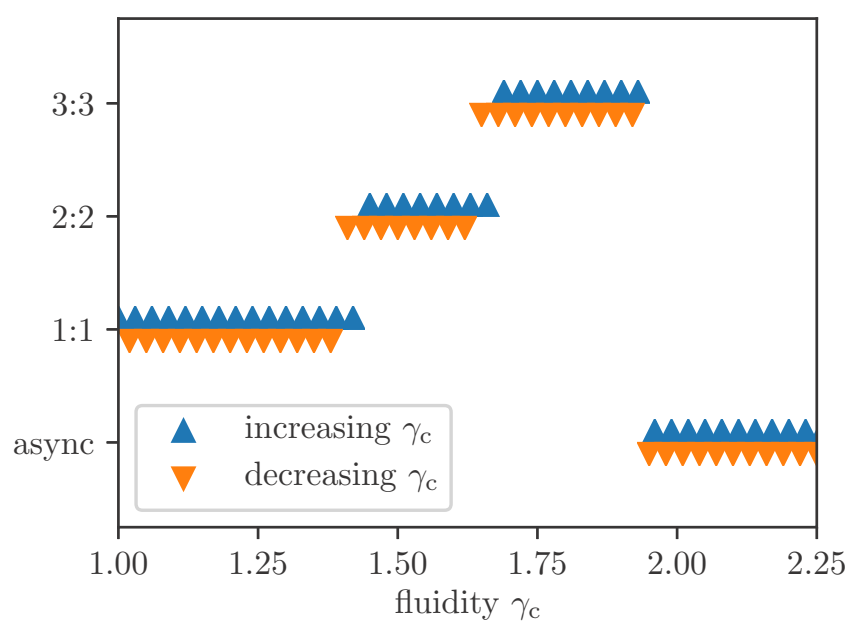

FIG. 10. At the boundaries between the asynchronous regime, $n: n$ synchronization, and $1: 1$ synchronization in Fig. 9, the two neighboring solutions coexist. There is hysteresis when increasing or decreasing $\gamma_{\mathrm{c}}$ at constant $k_{\mathrm{c}}=4$ (red line in Fig. 9).

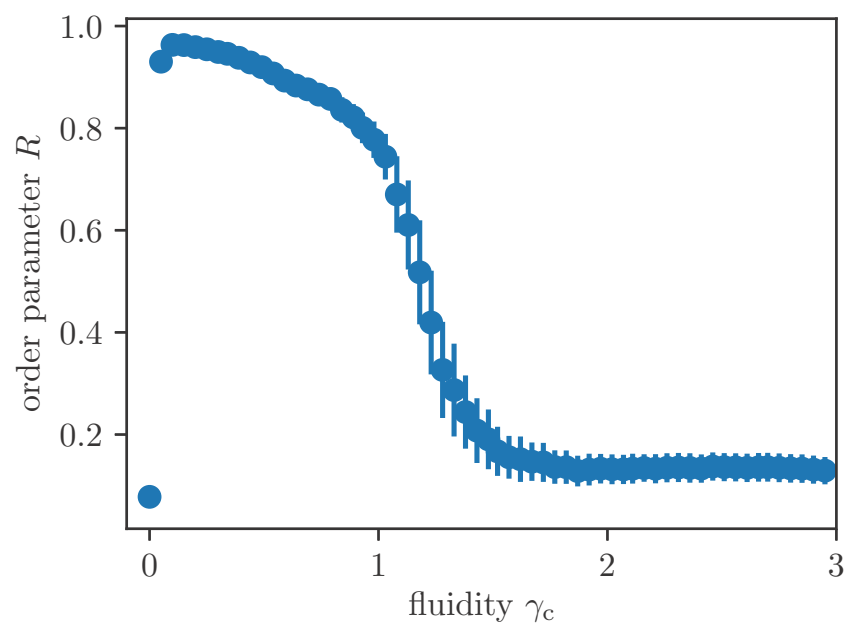

FIG. 11. Kuramoto order parameter $R$ of a linear chain of $N=$ 50 cells, averaged over the last $250 \mathrm{~s}$ of six different frequency distributions as function of fluidity $\gamma_{\mathrm{c}}$ at $k_{\mathrm{c}}=6$. Error bars indicate standard deviation. $R$ increases with decreasing $\gamma_{c}$ with the exception $\gamma_{\mathrm{c}}=0$ (see below). The chain is almost fully synchronized for small, nonzero $\gamma_{\mathrm{c}}$.

In the following, we investigate the dynamics of a chain of length $N=50$. The cells are initialized with random phases $\psi_{i}(0)$ distributed uniformly in $[-\pi, \pi)$; or, to be more precise, with electrical excitation $v_{i}(0), h_{i}(0)$ corresponding to the phase $\psi_{i}(0)$. Cell elongations, active forces, and viscous creeps are 0 at $t=0$. The system of ODEs is integrated over an overall period of $500 \mathrm{~s}$.

For six different frequency distributions the order parameter $R$ from Eq. (13) is calculated and averaged over the last $250 \mathrm{~s}$ of the simulation period. Figure 11 shows the resulting $R$ as function of the fluidity $\gamma_{\mathrm{c}}$ of the coupling springs averaged over all six realizations with error bars indicating the standard deviation. In the cases with $\gamma_{\mathrm{c}}>0, R$ clearly shows the transition from an asynchronous to a synchronous state as $\gamma_{\mathrm{c}}$ decreases. For $0<\gamma_{\mathrm{c}} \leqslant 1, R$ is close to 1 , for $\gamma_{\mathrm{c}} \geqslant$ $1.9, R$ is small and does not differ from the order parameter of the uncoupled system. Interestingly, in the synchronous regime, $R$ increases monotonously with decreasing $\gamma_{\mathrm{c}}$, but decreases again for $0.05 \geqslant \gamma_{\mathrm{c}}>0$ while remaining close to 1. Intermediate fluidities show an increased order parameter compared to the asynchronous cases; the shape of $R\left(\gamma_{\mathrm{c}}\right)$ in this regime also depends on the exact frequency distribution and initial condition which is visible in the relatively large standard deviation. As in the case of two cells, the purely elastic case $\gamma_{c}=0$ is special and will be discussed separately.

For the viscoelastic cases $\gamma_{c}>0$, the mean frequency of the cells, again averaged over the last $250 \mathrm{~s}$ and six different realizations, is shown in Fig. 12 as a function of fluidity. Here, too, the transition from asynchrony to synchrony with decreasing fluidity is visible. The average frequency increases as the slower cells in the initial frequency distribution are entrained by the faster ones. In the asynchronous regime there is no change in mean frequency and standard deviation. Towards lower fluidities, first the standard deviation and then the average frequency increases in the regime of intermediate $R$. Interestingly, the average frequency in the synchronous 


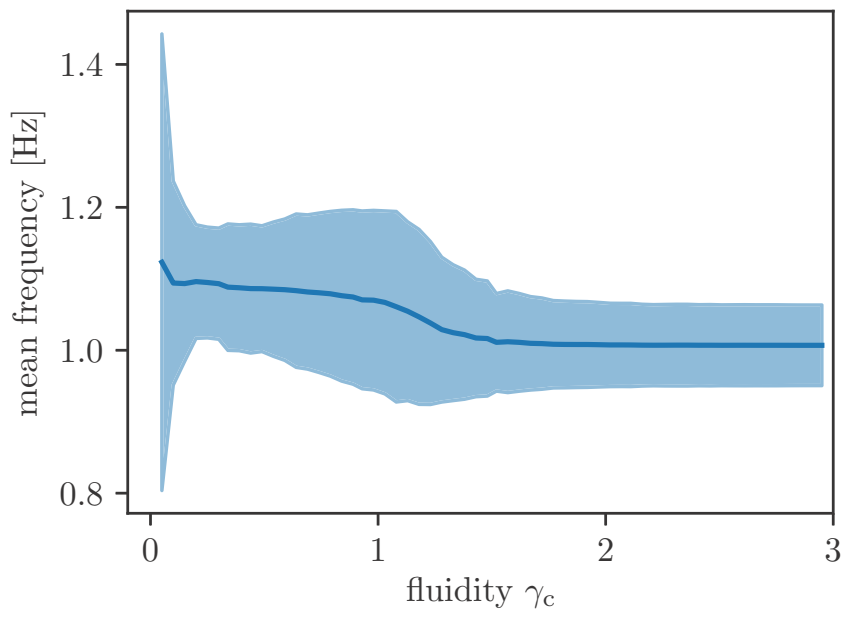

FIG. 12. The average frequency increases when the system approaches the synchronous regime. The shaded region indicates the standard deviation; only data for $\gamma_{\mathrm{c}}>0$ are shown.

regime is increased compared to the asynchronous cases but not as large as the largest frequencies in the initial distributions. Thus, it is the faster but not the fastest cells dominating the synchronized dynamics. As $R$ is slightly reduced in the case of $0<\gamma_{\mathrm{c}} \leqslant 0.05$, average frequency and frequency fluctuations both are increased.

To better understand the different regimes we now turn to exemplary space-time plots of the phases of each oscillator as function of time for different values of $\gamma_{c}$. An example of the synchronous regime in Fig. 11 at $\gamma_{c}=0.2$ is shown in Fig. 13(a). Almost all cells beat synchronously except for single cells whose natural frequencies are faster than the frequency of the synchronized population. For even smaller, nonzero $\gamma_{c}$ these fast cells are able to (at least partially) entrain their neighbors thus causing the increase in average frequency and frequency fluctuations at $0<\gamma_{\mathrm{c}} \leqslant 0.05$ together with the slight decrease in $R$ (data not shown). Figure 13(b) shows an

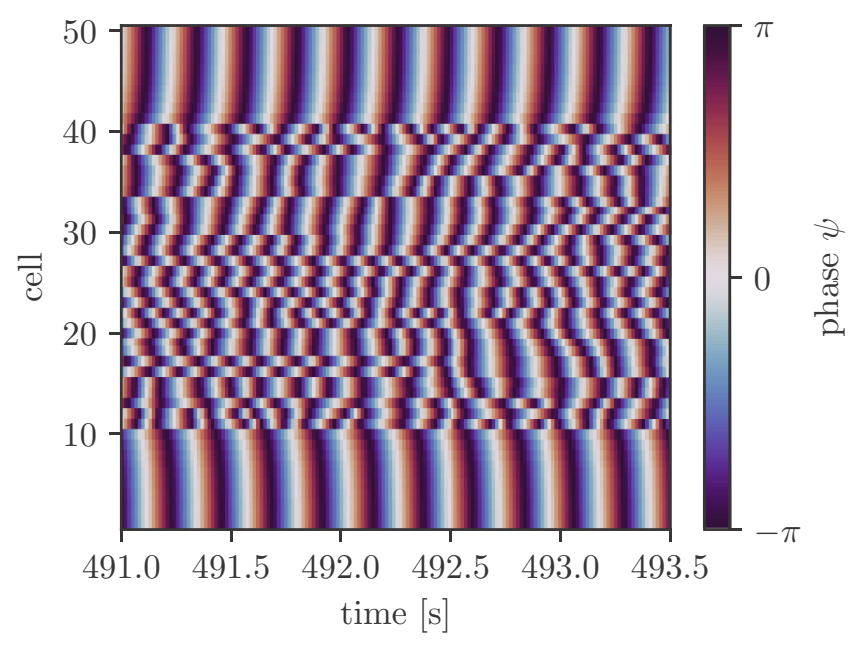

FIG. 14. Space-time plot of the purely elastic case $\gamma_{c}=0$ at $k_{\mathrm{c}}=6$ : The two populations at both ends of the chain synchronize with a constant phase difference of $\pi$ while the oscillators in between beat incoherently leading to an antiphase chimera state. Again the frequency is around $6 \mathrm{~Hz}$ and thus much larger than in the viscoelastic case.

example from the regime of intermediate order parameters at $\gamma_{\mathrm{c}}=1.33$. While not showing full synchronization there are areas of partially entrained cells where some but not all beats cause depolarization of neighboring cells - comparable to the $n: n$ case in the system of only two coupled cells.

As already mentioned, the case of purely elastic coupling $\gamma_{\mathrm{c}}=0$ is special, visible already in the drop of $R$ in Fig. 11 . A space-time plot of this situation is shown in Fig. 14 (note again, as in the two-cell case, the shorter time scale compared to Fig. 13). At the ends of the chain, there are two synchronized populations that remain phase-locked with a phase difference of $\pi$. So the cells inside one of the two populations are synchronized in phase among each other, while the two groups themselves are synchronized in antiphase. In between
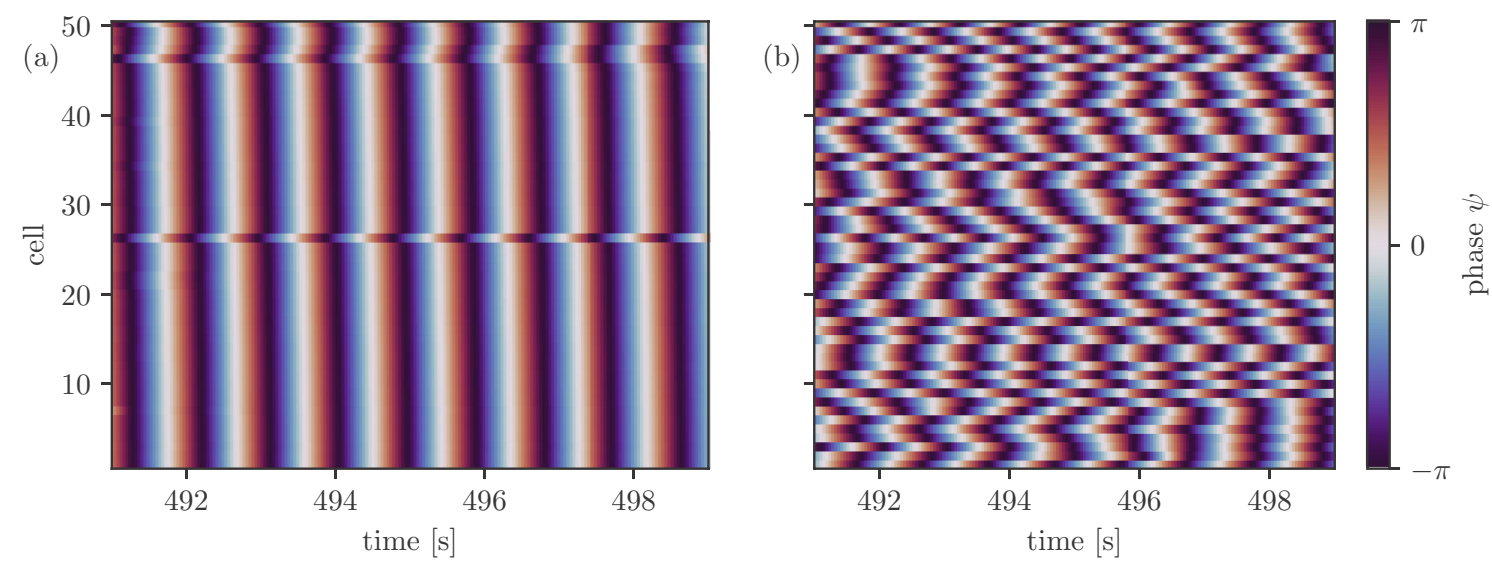

FIG. 13. Exemplary space-time plots showing the phases $\psi_{i}$ of each of the $N=50$ oscillators color-coded as function of time for $k_{\mathrm{c}}=6$. (a) Case of $\gamma_{\mathrm{c}}=0.2$ where almost all cells beat synchronously at $1.1 \mathrm{~Hz}$. Notably, the cells that are not (fully) entrained are the ones with the largest natural frequencies. (b) At intermediate fluidities $\gamma_{\mathrm{c}}=1.33$ the system does not display synchronization clearly, but shows regions of cells that periodically remain close to each other for a few oscillations. This corresponds to a generalized version of the $n: n$ (or $n: m$ ) synchronization in the two-cell system. 


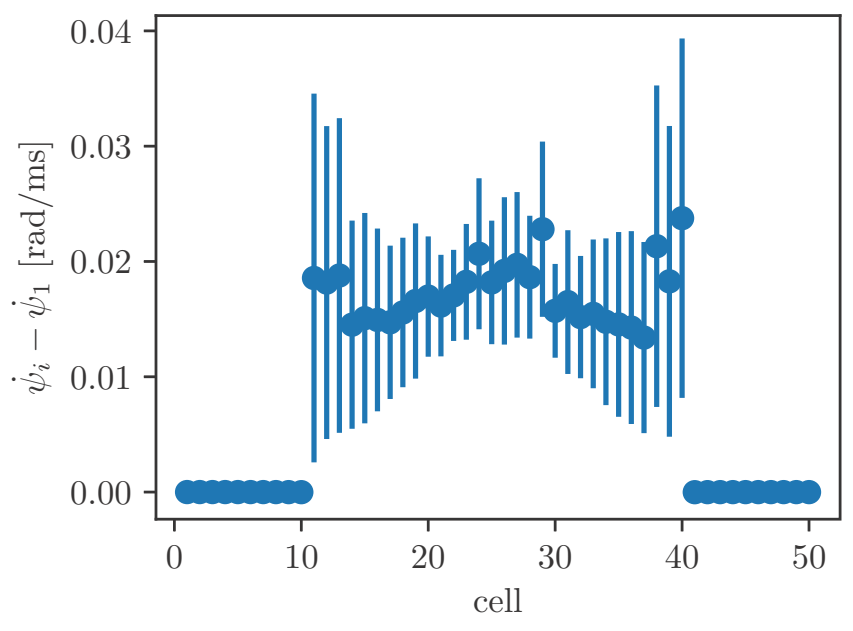

FIG. 15. In the antiphase chimera state, the average phase velocity $\dot{\psi}_{i}-\dot{\psi}_{1}$ in the frame of reference of the first cell vanishes in the two synchronized regions. It is nonzero only for the incoherent group in the center.

the two groups, the cells are not synchronized at all and beat incoherently, leading two a chimera state with antiphase synchronization in the coherent part that we denote as the antiphase chimera state [15]. The chimera state also is visible when looking at the time average of the phase velocity $\dot{\psi}_{i}$ of each velocity in the comoving frame of reference of the first cell, i.e., one of the two synchronous populations shown in Fig. 15 averaged over the last $100 \mathrm{~s}$ of the simulation period with standard deviation as error bars. The relative phase velocity $\dot{\psi}_{i}-\dot{\psi}_{1}$ is 0 in both coherent groups and larger than 0 in the incoherent population.

\section{DISCUSSION}

We observed synchronization of mechanically coupled excitable oscillators in the case of a simple model for the excitation-contraction dynamics of cardiomyocytes embedded in a viscoelastic extracellular matrix. The system of two cells shows essentially uncoupled dynamics for large fluidities $\gamma_{\mathrm{c}}$ and low elasticities $k_{\mathrm{c}}$ of the coupling Maxwell element. This was to be expected as all deformation by the cells' contraction is essentially absorbed by the viscous creep of the Maxwell element. For stiffer, less fluid coupling the cells are able to influence each other via deformation and resulting stretch-activated currents leading to $n: n$ and, for even stiffer coupling to $1: 1$ synchronization. The overall dynamics in all these cases is determined by the faster of the two cells, that either fully or partially entrains the slower cell. For any $\gamma_{c}>0$ the faster cell (after possible transients) is unperturbed by the beating of the slower one. This prevailing of the faster cell again could perhaps be expected as a consequence of the excitable nature of the two oscillators. After having been excited by a hyperthreshold stretch-activated current transmitted by the faster cell the slower one cannot be directly excited again by its internal dynamics. The fast cell on the other hand then is contracted and either excited or refractory when it is reached by the deformation caused by the slower cell that in this state cannot influence the dynamics of the fast cell.
The case of purely elastic coupling $\gamma_{\mathrm{c}}=0$ is special in the sense that here, for sufficiently large $k_{\mathrm{c}}$, the original frequencies of both cells do not play a role. Instead, as soon as one cell contracts, it immediately causes a hyperthreshold stimulus in the other one which then depolarizes and contracts in turn, leading to the observed antiphase synchrony. The frequency is given by the elasticity of the coupling spring.

The linear chain expresses the same general features: at given elasticity $k_{\mathrm{c}}$, high fluidity corresponds to an essentially decoupled system, while at low fluidity almost all oscillators synchronize at a common frequency. Interestingly, the cells that are not entrained in these almost completely synchronized cases beat faster than the synchronized group-thus, the synchronized dynamics here is not necessarily dominated by the fastest oscillators in the system. Further decreasing fluidity however increases the influence of these fastest cells on their neighbors, leading to a small drop in the Kuramoto order parameter $R$ of the whole system. At very small $\gamma_{\mathrm{c}}>0$, we expect to see full synchronization again at the frequency of the fastest cell. In analogy to the $n: n$ synchrony in the system of two cells, there is a regime of intermediate fluidities, where only some but not all beats of the cells are induced by hyperthreshold stretch-activated currents so there is no overall synchrony. Still the order parameter of the total system is increased compared to the asynchronous case. With decreasing $\gamma_{\mathrm{c}}$ in this regime more and more beats are induced by the neighboring cells' contractions until synchronous groups emerge.

Again, purely elastic coupling is separated from any case $\gamma_{\mathrm{c}}>0$. We still see the antiphase synchronization from the system of two oscillators; now in the antiphase synchrony of the two groups at the ends of the chain. In each of the two groups the cells are synchronized in phase. The two groups are separated by a population of incoherently oscillating cells with higher average phase velocity, leading to the observed antiphase chimera state. Each oscillator is only coupled to its next neighbors, so there is only local coupling in our system. Since we use fixed boundary conditions our results differ from the chimera states found by Laing [16] where the author finds chimera states in locally coupled reaction-diffusion systems. While our purely elastic coupling can essentially be interpreted as a discrete form of diffusive coupling, Laing uses periodic boundary conditions and observe a single coherent in-phase group apart from an incoherent population of oscillators. The chimera state shown here stems from the same initial frequency distribution as in the cases $\gamma_{c}>0$, i.e., from a normal distribution around $1 \mathrm{~Hz}$ with a standard deviation of $0.05 \mathrm{~Hz}$. We observe the same antiphase chimera also for a chain of identical oscillators with natural frequencies of $1 \mathrm{~Hz}$ only differing in their initial phases.

\section{CONCLUSION}

We investigated the possible synchronization of viscoelastically coupled excitable oscillators both in systems of two and of a linear chain of these oscillators. We found qualitatively different results between purely elastic $\left(\gamma_{c}=0\right)$ and viscoelastic $\left(\gamma_{\mathrm{c}}>0\right)$ coupling. In the latter case we find a transition to $1: 1$ synchronization of the coupled cell at the frequencies of the faster oscillators as the coupling 
element becomes stiffer (increasing elasticity $k_{\mathrm{c}}$, decreasing fluidity $\gamma_{\mathrm{c}}$ ).

In an experimental study [5] of the rheological properties of the extracellular matrix in the initial stages of engineered heart muscle we found that over time, the extracellular matrix (ECM) becomes stiffer and less fluid, a process that is further amplified by the presence of fibroblasts (cf. Fig. 4 in [5]). Considering the beating cardiomyocytes as excitable oscillators with the ECM providing viscoelastic coupling, our numerical results presented here suggest that during aging, qualitatively, the ECM evolves to a parameter regime allowing for synchronization of the cells. Additionally, it was experimentally observed that the presence of fibroblasts is necessary for the successful growth of engineered heart muscle [3]. The simulations presented are consistent with this feature because in the presence of fibroblasts, the stiffening of the ECM over time is even more pronounced [5]. Thus, the ideal ECM for the growth of engineered heart muscle is also the one most likely to allow for mechanical synchronization of the cardiomyocytes, even without electrochemical coupling via gap junctions.

In the case of purely elastic coupling, we observe antiphasic $-1: 1$ synchronization. In the system with only two oscillators, the cells themselves synchronize in antiphase; in the linear chain this is extended to the antiphase chimera state with two antiphasic groups at the ends of the chain, each of which consist of in-phase synchronized oscillators. Since each oscillator is only coupled to its direct neighbors, this is one example of a chimera state in a system with purely local coupling as described, for example in [16].

\section{ACKNOWLEDGMENTS}

We acknowledge financial support from the German Research Foundation (DFG) via the Collaborative Research Center SFB 937, Project No. A18. F.S. acknowledges support by the Young DZHK program by the DZHK (German Center for Cardiovascular Research). S.L. acknowledges support through the DZHK (German Center for Cardiovascular Research), Partner Site Göttingen. The authors also thank F. Rehfeldt, University of Göttingen, and W.-H. Zimmermann,
M. Tiburcy, and S. F. Schlick, Institute of Pharmacology and Toxicology for interesting discussions about viscoelastic and dynamical properties of cardiomyocytes in the extracellular matrix.

\section{APPENDIX A: EQUATIONS OF MOTIONS OF A LINEAR CHAIN}

The dynamics of the linear chain of $N$ viscoelastically coupled excitable cells require a more extensive description than the system of just two cells. On the one hand, each of the $N$ cells possesses its own excitation contraction dynamics with excitation variables $v_{i}, h_{i}$, active contractile force $T_{i}$, and stretch-activated current $I_{\mathrm{S}, i}, i \in\{1, N\}$. All cells have an (arbitrary) resting length $x_{i}^{0}$, an elongation $\Delta x_{i}$, and a resulting restoring force $F_{\mathrm{r}, i}=-k_{\mathrm{r}} \Delta x_{i}-\gamma_{\mathrm{r}} \frac{d \Delta x_{i}}{d t}$. To describe the dynamics of the cell elongations and matrix deformations on the other hand, we consider $2 N$ mass points of equal arbitrary mass $m=1$ located at the cell boundaries. Including the connections to the boundaries of the system, there are $N+$ 1 Maxwell elements with (again, arbitrary) resting length $l_{j}^{0}$ and viscous creep $d_{j}, j \in\{1, N+1\}$. The $2 N$ mass points are located at positions $\xi_{\alpha}, \alpha \in\{1,2 N\}$. Thus, the cell elongation of cell $i$ is

$$
\Delta x_{i}=\left|\xi_{2 i}-\xi_{2 i-1}\right|-x_{i}^{0} .
$$

The viscous creep of the $j$ th Maxwell element now obeys the ODE,

$$
\frac{d d_{j}}{d t}=-\gamma_{\mathrm{c}}\left(\xi_{2 j-1}-\xi_{2 j-2}-l_{j}^{0}+d_{j}\right)
$$

where $\xi_{0}=0$ and $\xi_{2 N+1}=\sum_{i=1}^{N} x_{i}^{0}+\sum_{j=1}^{N+1} l_{j}^{0}=: L$ are kept fixed. The force due to the deformation of this element is then

$$
F_{\mathrm{c}, j}=-k_{\mathrm{c}}\left(\xi_{2 j-1}-\xi_{2 j-2}-l_{j}^{0}+d_{j}\right) .
$$

The resulting equations of motion for the positions of the mass points are

$$
m \frac{d^{2} \xi_{\alpha}}{d t^{2}}=\frac{1}{2}\left(F_{\mathrm{r}, \alpha / 2}-T_{\alpha / 2}\right)-\frac{1}{2} F_{\mathrm{c}, \alpha / 2+1}
$$

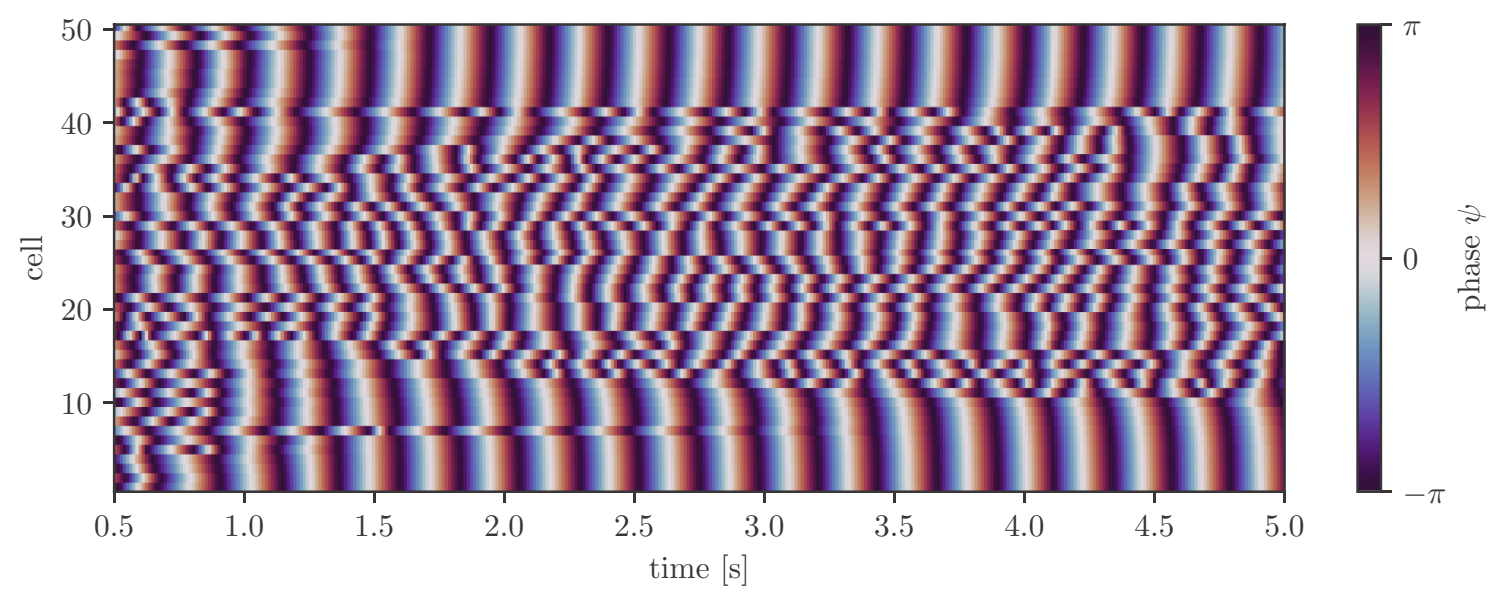

FIG. 16. The initial transients leading to the antiphase chimera state in a linear chain of $N=50$ cells at $k_{\mathrm{c}}=6, \gamma_{\mathrm{c}}=0$. Already after $4 \mathrm{~s}$, the initially random phases have reached the chimera state with two coherent groups synchronized in antiphase. 


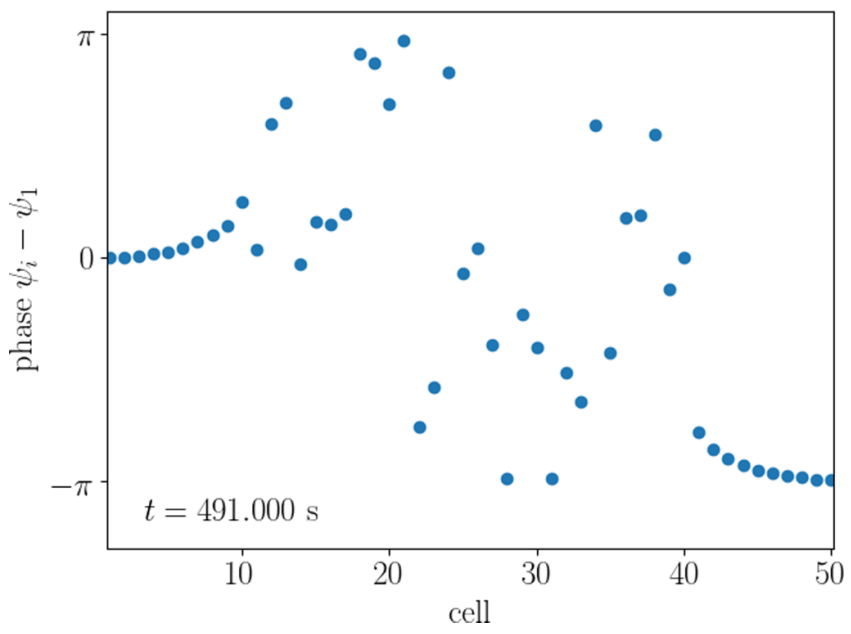

FIG. 17. The supplementary movie [15] shows the time evolution of the phase of each oscillator $\psi_{i}$ in the frame of reference of the first cell from $t=491 \mathrm{~s}$ to $t=493.5 \mathrm{~s}$. The phases in the left group remain close to 0 , the group to the right is locked close to $-\pi$. The cells in between oscillate incoherently. for $\alpha$ even and

$$
m \frac{d^{2} \xi_{\alpha}}{d t^{2}}=-\frac{1}{2}\left(F_{\mathrm{r},(\alpha+1) / 2}-T_{(\alpha+1) / 2}\right)+\frac{1}{2} F_{\mathrm{c},(\alpha+1) / 2}
$$

for $\alpha$ odd. Solving Eqs. (A4) and (A5) yields the cell elongations $\Delta x_{i}$ via Eq. (A1) that then are used to calculate $I_{\mathrm{S}, i}$ and, finally, solve the ODEs for $v_{i}, h_{i}$, and $T_{i}$ as described in the case of two cells. Note that the system now is not explicitly overdamped anymore since it cannot be solved explicitly for $\frac{d \xi_{\alpha}}{d t}$ in the overdamped case. However, the choice of a large $\gamma_{\mathrm{r}}$ in comparison to $m\left(\gamma_{\mathrm{r}}=30\right.$ in our case $)$, ensures that the resulting relaxation of each cell effectively is still overdamped.

\section{APPENDIX B: INITIAL TRANSIENT OF THE ANTIPHASE CHIMERA STATE}

Figure 16 shows the initial transient leading from random initial phases to the antiphase chimera state. Already after $4 \mathrm{~s}$ the chimera state is visible and remains persistent for the remainder of the 500-s simulation period (see Fig. 17 and supplemental movie [15]).
[1] D. M. Bers, Cardiac excitation-contraction coupling, Nature (London) 415, 198 (2002).

[2] B. Fujita, M. Tiburcy, S. Ensminger, and W.-H. Zimmermann, State-of-the-art in tissue-engineered heart repair, in Cardiac Regeneration, Cardiac and Vascular Biology (Springer, Cham, 2017), pp. 219-239.

[3] M. Tiburcy, J. E. Hudson, P. Balfanz, S. F. Schlick, T. Meyer, M.-L. C. Liao, E. Levent, F. Raad, S. Zeidler, E. Wingender, J. Riegler, M. Wang, J. D. Gold, I. Kehat, E. Wettwer, U. Ravens, P. Dierickx, L. V. Laake, M.-J. Goumans, S. Khadjeh, et al., Defined engineered human myocardium with advanced maturation for applications in heart failure modelling and repair, Circulation 135, 1832 (2017).

[4] M. Tiburcy, T. Meyer, P. L. Soong, and W.-H. Zimmermann, Collagen-based engineered heart muscle, in Cardiac Tissue Engineering: Methods and Protocols, Methods in Molecular Biology, edited by M. Radisic and L. D. Black III (Springer, New York, 2014), pp. 167-176.

[5] S. F. Schlick, F. Spreckelsen, M. Tiburcy, L. M. Iyer, T. Meyer, L. C. Zelarayan, S. Luther, U. Parlitz, W.-H. Zimmermann, and F. Rehfeldt, Agonistic and antagonistic roles of fibroblasts and cardiomyocytes on viscoelastic stiffening of engineered human myocardium, Prog. Biophys. Mol. Biol. 144, 51 (2019).

[6] H. Hu and F. Sachs, Stretch-activated ion channels in the heart, J. Mol. Cell. Cardiol. 29, 1511 (1997).

[7] Y. H. Zhang, J. B. Youm, H. K. Sung, S. H. Lee, S. Y. Ryu, S.-H. Lee, W.-K. Ho, and Y. E. Earm, Stretch-activated and background non-selective cation channels in rat atrial myocytes, J. Physiol. 523, 607 (2000).
[8] I. Nitsan, S. Drori, Y. E. Lewis, S. Cohen, and S. Tzlil, Mechanical communication in cardiac cell synchronized beating, Nat. Phys. 12, 472 (2016).

[9] S. Stein, S. Luther, and U. Parlitz, Impact of viscoelastic coupling on the synchronization of symmetric and asymmetric self-sustained oscillators, New J. Phys. 19, 063040 (2017).

[10] C. C. Mitchell and D. G. Schaeffer, A two-current model for the dynamics of cardiac membrane, Bull. Math. Biol. 65, 767 (2003).

[11] K. Djabella, M. Landau, and M. Sorine, A two-variable model of cardiac action potential with controlled pacemaker activity and ionic current interpretation, in 2007 46th IEEE Conference on Decision and Control, New Orleans, LA, USA (IEEE, 2007), pp. 5186-5191.

[12] L. D. Weise and A. V. Panfilov, New mechanism of spiral wave initiation in a reaction-diffusion-mechanics system, PLOS One 6, e27264 (2011).

[13] L. D. Weise and A. V. Panfilov, Emergence of Spiral Wave Activity in a Mechanically Heterogeneous Reaction-DiffusionMechanics System, Phys. Rev. Lett. 108, 228104 (2012).

[14] Y. Kuramoto, Mutual entrainment, in Chemical Oscillations, Waves, and Turbulence, Springer Series in Synergetics, edited by Y. Kuramoto (Springer, Berlin, Heidelberg, 1984), pp. $60-88$.

[15] See Supplemental Material at http://link.aps.org/supplemental/ 10.1103/PhysRevE.100.032214 for an animation of the phase of each oscillator in the antiphase chimera state.

[16] C. R. Laing, Chimeras in networks with purely local coupling, Phys. Rev. E 92, 050904(R) (2015). 


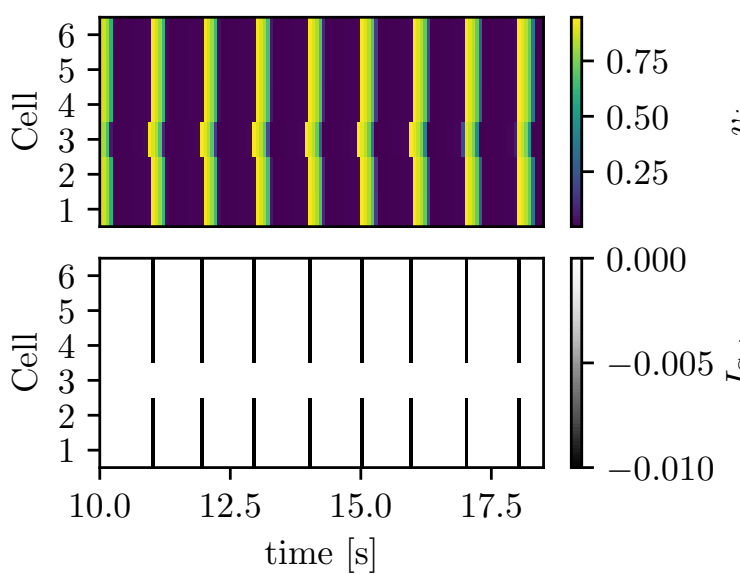

(a) $\gamma_{\mathrm{c}}=0.1$
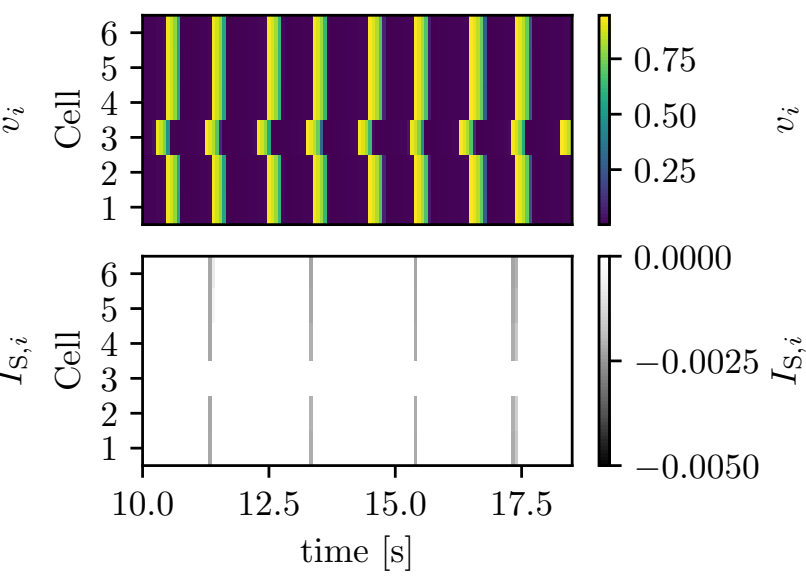

(b) $\gamma_{c}=0.5$

Figure 3.1 1:1 and 2:2 synchronization in a short chain with one faster (cell $3, \nu_{3}=1 \mathrm{~Hz}$ ) and 5 identical slower cells with natural frequency $0.9 \mathrm{~Hz}$. The upper panel shows the membrane voltage $v_{i}$ of each cells color-coded as a function of time, the lower panels show the stretch-activated current $I_{\mathrm{S}, i}$. As in the system with two cells in section 3.1, for fixed elasticity $k_{\mathrm{c}}=4$, the fast cell entrains the whole system at low fluidity (a) $\gamma_{\mathrm{c}}=0.1$. Each beat of the slow cells is triggered by a hyperthreshold SAC. At higher fluidity $\gamma_{\mathrm{c}}=0.5(\mathrm{~b})$, the short chain, too, shows 2:2 synchronization. Note the different scales for $I_{\mathrm{S}, i}$ between (a) and (b).

\subsection{Further results on synchronization in short lin- ear chains of excitable oscillators}

The linear chains of viscoelastically coupled excitable oscillators presented in the previous section (or in [33], section III.B) with random frequencies show a transition to almost full synchronization as fluidity $\gamma_{\mathrm{c}}$ is decreased at constant elasticity $k_{\mathrm{c}}$. In contrast to the system of only two coupled cells, the fastest cell does not necessarily dominate the dynamics even in the case of highest Kuramoto order parameter $R$, i.e., the most synchronous cases. In order to better understand this behavior, it helps to consider a simpler system of a short chain with $N=6$ cells. As in the two-cell system in the previous section, there is one fast cell (at the third position in the chain) with a natural frequency $\nu_{3}=1 \mathrm{~Hz}$, the other five cells are slower with $\nu_{i}=0.9 \mathrm{~Hz}$. The initial phases are identical and correspond to the resting state of the Mitchell-Schaeffer model; $v_{i}(0)=0, h_{i}(0)=1$. The elasticity of the coupling springs is kept constant at $k_{\mathrm{c}}=4$, only the fluidity $\gamma_{\mathrm{c}}$ is varied between 0.1 and 1 . In every other aspect system is identical to the longer chain with $N=50$ in section 3.1.

Figure 3.1 shows two examples of the dynamics of the membrane potentials of the cells in such a short chain very similar to $1: 1$ and $n: n$ synchrony explained in the two-cell system in the previous section. For small $\gamma_{\mathrm{c}}=0.1$, figure $3.1 \mathrm{a}$ shows the membrane potential $v_{i}$ of each cell in the upper and stretch-activated currents $I_{\mathrm{S}, i}$ in the lower panel. As in the 1:1 synchronization with two cells, the fast one fully entrains 


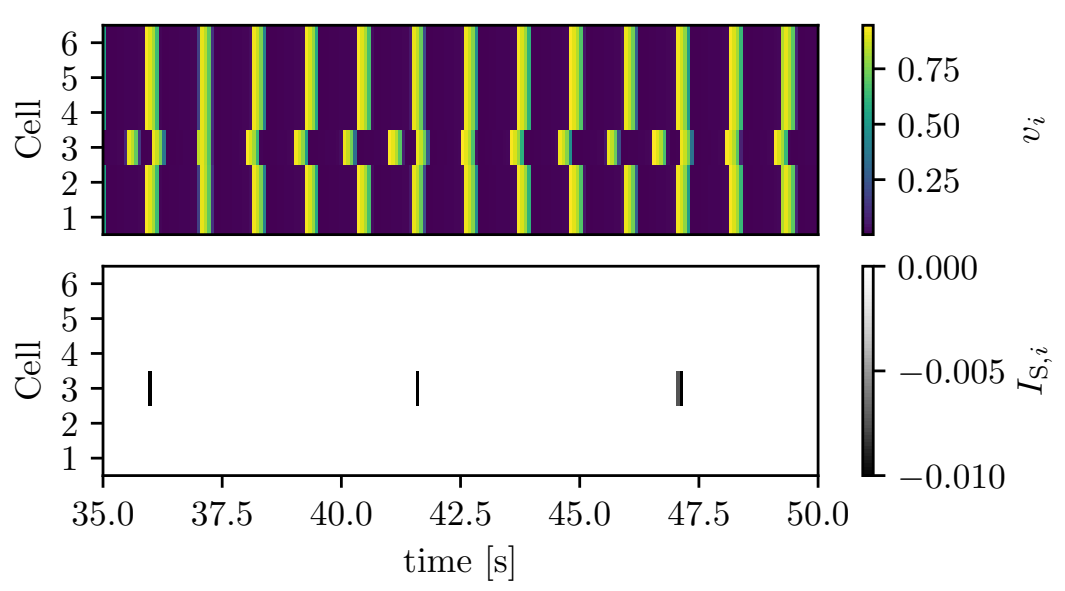

Figure 3.2 Membrane voltage $v_{i}$ and stretch-activated current $I_{\mathrm{S}, i}$ of a short chain at elasticity $k_{\mathrm{c}}=4$ and fluidity $\gamma_{\mathrm{c}}=1$. In contrast to the case of only two cells, the slow cells dominate the dynamics. The system exhibits $6: 5$ synchronization where every sixth beat of the fast cell is triggered by a hyperthreshold SAC caused by the contraction of the slow cells.

the slow ones. Each action potential of the slow cells is triggered by a hyperthreshold SAC stemming from the deformation due to the contraction of the fast cell. The case displayed in figure $3.1 \mathrm{~b}$ for $\gamma_{\mathrm{c}}=0.5$ is the corresponding case of $2: 2$ synchronization; now between the fast cell and the group of slow cells. The mechanism is the same as before; the electrical and mechanical configuration only allows for hyperthreshold $\mathrm{SAC}$ in the slow cells at every other beat. Compared to the system with two cells, the transition from 1:1 to 2:2 happens at smaller fluidity (cf. figure 9 in [33]). In all cases, the fast cell dominates the dynamics.

This changes when fluidity is increased further as shown in figure 3.2 for $\gamma_{\mathrm{c}}=1$. The system exhibits 6:5 synchronization in this case whereby every sixth beat of the fast cell is driven by the slow cells that, in turn, remain unperturbed by the fast one. The slower cells thus dominate the system, at least for a certain range of coupling parameters, which is in contrast to the common understanding of excitable systems where one usually expects the fastest source to prevail.

The cases above illustrate how in chains of viscoelastically coupled excitable oscillators, depending on the details of the coupling, a large population of slower cells may dominate over few fast ones. When fluidity is reduced, however, the influence of the fast cells grows. This grown influence on the slower cells may even decrease the overall order in the system as the decrease in $R$ in figure 11 in the previous section shows when comparing $\gamma_{c}=0.2$ and $\gamma_{c}=0.1$ while at the same time, average frequency and its fluctuations increase (figure 12 in the previous section). 



\section{Chapter 4}

\section{Rheological properties of the extracellular matrix}

\subsection{Development of the ECM in the presence of cardiomyocytes and nonmyocytes}

The following section consists of the article [1] published as

S. F. Schlick, F. Spreckelsen, M. Tiburcy, L. M. Iyer, T. Meyer, L. C. Zelarayan, S. Luther, U. Parlitz, W.-H. Zimmermann, and F. Rehfeldt. "Agonistic and antagonistic roles of fibroblasts and cardiomyocytes on viscoelastic stiffening of engineered human myocardium". In: Progress in Biophysics and Molecular Biology 144 (July 2019), pp. 51-60. DOI: 10.1016/j.pbiomolbio.2018.11.011

under the Creative Commons Attribution License (CC BY 4.0) ${ }^{1}$ of which Susanne Schlick and I are equally contributing first authors.

My contributions mainly concern the ECM rheology results. Florian Rehfeldt, Susanne Schlick and I devised the rheological experiments; Susanne and I performed them. The analysis of the rheological data was performed by me; I created the corresponding figures 3, 4, 6, and 7. Ulrich Parlitz and I devised the mathematical ad-hoc model used to reproduce the nonlinear stress response of the ECM in LAOS measurements. All authors contributed to the writing of the article; Florian Rehfeldt and I wrote the result sections 2.3, 2.6, and 2.7 on linear and non-linear ECM rheology. Note that in the article, (human foreskin) fibroblasts are abbreviated as "Fib", not as $\mathrm{HFF}$ as in the remainder of this thesis.

All hydrogels used for the experiments in this studies were prepared using the EHM protocol described in section 2.4.2. Rheometry included an initial time sweep of length $T_{\mathrm{TS}}=60 \mathrm{~min}$ and sine LAOS at $1 \mathrm{~Hz}$ with amplitudes up to $\gamma_{0}=10 \%$ as described in section 2.4.3. Table 3 of the supplementary material to this article containing the coefficients for the ad-hoc model has been reproduced in appendix C.1.

\footnotetext{
${ }^{1}$ https://creativecommons.org/licenses/by/4.0/
} 


\title{
Agonistic and antagonistic roles of fibroblasts and cardiomyocytes on viscoelastic stiffening of engineered human myocardium
}

\author{
Susanne F. Schlick a, b, 1 , Florian Spreckelsen b, c, d, 1, Malte Tiburcy ${ }^{\text {a, b }}{ }^{\text {, Lavanya M. Iyer }}{ }^{\text {a, b }}$, \\ Tim Meyer ${ }^{\text {a, b }}$, Laura C. Zelarayan ${ }^{\text {a, b }}$, Stefan Luther ${ }^{\text {a, b, c, d, Ulrich Parlitz }}{ }^{\text {b, c, d }}$, \\ Wolfram-Hubertus Zimmermann ${ }^{\text {a, b, * }}$, Florian Rehfeldt ${ }^{\text {e, ** }}$ \\ a Institute of Pharmacology and Toxicology, University Medical Center Göttingen, 37075, Göttingen, Germany \\ b DZHK (German Center for Cardiovascular Research), partner site Göttingen, 37075, Göttingen, Germany \\ ${ }^{c}$ University of Göttingen, Institute for Nonlinear Dynamics, 37077, Göttingen, Germany \\ ${ }^{d}$ Max Planck Institute for Dynamics and Self-Organization, 37077, Göttingen, Germany \\ e University of Göttingen, 3rd Institute of Physics - Biophysics, 37077, Göttingen, Germany
}

\section{A R T I C L E I N F O}

\section{Article history:}

Received 17 July 2018

Received in revised form

2 November 2018

Accepted 27 November 2018

Available online 12 December 2018

\section{Keywords:}

Engineered heart muscle

Fibroblasts

Cardiomyocytes

Collagen

Rheology

Nonlinear mechanics

\begin{abstract}
A B S T R A C T
Cardiomyocyte and stroma cell cross-talk is essential for the formation of collagen-based engineered heart muscle, including engineered human myocardium (EHM). Fibroblasts are a main component of the myocardial stroma. We hypothesize that fibroblasts, by compacting the surrounding collagen network, support the self-organization of cardiomyocytes into a functional syncytium. With a focus on early selforganization processes in EHM, we studied the molecular and biophysical adaptations mediated by defined populations of fibroblasts and embryonic stem cell-derived cardiomyocytes in a collagen type I hydrogel. After a short phase of cell-independent collagen gelation $(30 \mathrm{~min})$, tissue compaction was progressively mediated by fibroblasts. Fibroblast-mediated tissue stiffening was attenuated in the presence of cardiomyocytes allowing for the assembly of stably contracting, force-generating EHM within 4 weeks. Comparative RNA-sequencing data corroborated that fibroblasts are particularly sensitive to the tissue compaction process, resulting in the fast activation of transcription profiles, supporting heart muscle development and extracellular matrix synthesis. Large amplitude oscillatory shear (LAOS) measurements revealed nonlinear strain stiffening at physiological strain amplitudes $(>2 \%)$, which was reduced in the presence of cells. The nonlinear stress-strain response could be characterized by a mathematical model. Collectively, our study defines the interplay between fibroblasts and cardiomyocytes during human heart muscle self-organization in vitro and underscores the relevance of fibroblasts in the biological engineering of a cardiomyogenesis-supporting viscoelastic stroma. We anticipate that the established mathematical model will facilitate future attempts to optimize EHM for in vitro (disease modelling) and in vivo applications (heart repair).
\end{abstract}

๑ 2018 The Authors. Published by Elsevier Ltd. This is an open access article under the CC BY license (http://creativecommons.org/licenses/by/4.0/).

\footnotetext{
Abbreviations: Cardiomyocytes, (CMs); human foreskin fibroblasts, (Fibs); engineered human myocardium, (EHM); large amplitude oscillatory shear, (LAOS). * Corresponding author. Institute of Pharmacology and Toxicology, University Medical Center Göttingen, 37075, Göttingen, Germany.

** Corresponding author. University of Göttingen, 3rd Institute of Physics Biophysics, 37077, Göttingen, Germany.

E-mail addresses: w.zimmermann@med.uni-goettingen.de (W.-H. Zimmermann), rehfeldt@physik3.gwdg.de (F. Rehfeldt).

1 equally contributing first authors.
}

\section{Introduction}

Engineered human myocardium (EHM) presents structural, functional, and molecular properties of bona fide postnatal heart muscle (Tiburcy et al., 2017). Its advanced degree of organotypic maturation is exemplified by an anisotropic sarcomere assembly with ultrastructural evidence for the formation of M-bands as well as physiological responses to preload (Frank-Starling mechanism), stimulation frequency (Bowditch phenomenon), and pharmacological stimuli such as catecholamines. For EHM assembly, cardiomyocytes and fibroblasts are embedded in a collagen type I hydrogel and cast into preformed molds of suitable geometries. 
After the onset of spontaneous beating (within $72 \mathrm{~h}$ ) EHM are subjected to mechanical loading to further enhance maturation (Tiburcy et al., 2017). Additional means to enhance tissue maturation include the use of electrical stimuli at (near-)physiological frequencies (Godier-Furnémont et al., 2015; Ronaldson-Bouchard et al., 2018).

The use of defined cardiomyocyte and fibroblast populations mixed at an optimized ratio in a collagen hydrogel environment and exposure to mechanical loading to facilitate auxotonic contractions are key for the heart muscle self-assembly process (Tiburcy et al., 2017). The pivotal role of the initially less appreciated non-cardiomyocyte stroma cells, and here in particular fibroblasts, has now clearly been recognized (Kensah et al., 2013; Naito et al., 2006; Soong et al., 2012; Zhang et al., 2013). Alternatively, endothelial cells (Weinberger et al., 2016) or so called tri-culture models comprising cardiomyocytes, smooth muscle cells, and endothelial cells (Gao et al., 2018) have been developed. In each of these assemblies it is likely that the non-myocyte component exhibits some fibroblast-like activity, for example by epithelial/endothelialmesenchymal transformation (EMT/EndMT (Li et al., 2018)) or cellular impurities in the applied cell populations.

The specific mode(s) of action underlying fibroblast-supported heart muscle assembly remain elusive, but may include the synthesis of cardiomyogenic extracellular matrix, paracrine crosstalk, and biomechanical stimuli mediated via biophysical interactions with the extracellular matrix. Moreover, it is quite likely that these processes occur in a time-sensitive manner. In this context, the process of early tissue consolidation, which leads to a macroscopically visible collagen hydrogel compaction within $1 \mathrm{~h}$ after casting of the EHM reconstitution mixture, is least understood (Tiburcy et al., 2014); compromised early tissue compaction is indicative for poor outcome, i.e., a failure to form force-generating EHM (systolic force amplitude: $>1 \mathrm{mN} / \mathrm{mm}^{2}$ ) within a timeframe of two to four weeks (Tiburcy et al., 2017).

In this study, we sought to determine the factors governing collagen hydrogel consolidation with a particular focus on in situ mechanical quantification of early EHM compaction as well as the specific roles of fibroblasts and cardiomyocytes during this process. We used rheological measurements to define distinct phases of cell-independent collagen gelation and cell-dependent collagen compaction. Here, we defined fibroblasts as the main contributors to collagen matrix stiffening and cardiomyocytes as "cellular antagonists" of this process. Since most biopolymers and biological tissues exhibit a nonlinear viscoelastic behavior (Storm et al., 2005), we also performed large amplitude oscillatory shear (LAOS (Ewoldt et al., 2008)) measurements to determine the cellular impact on strain-stiffening of the consolidated tissue. Finally, we propose a mathematical model characterizing mechanical stress as a function of strain and strain rate, which we view as an important step for future numerical simulations of cell-matrix interactions during EHM formation.

\section{Results}

\subsection{Engineered human myocardium input cell populations and development}

Engineered human myocardium (EHM; Fig. 1A) was generated by casting defined mixtures of collagen type I, human embryonic stem cell-derived cardiomyocytes (CMs), and human foreskin fibroblasts (Fibs) in circular molds; reconstitution mixtures comprising CMs and Fibs at a 2:1 ratio were recently identified to optimally support EHM formation (Tiburcy et al., 2017). We controlled CM (alpha-actinin: $95 \pm 2 \% ; \mathrm{n}=3$ ) and Fib (CD90: $94 \pm 5 \%, n=3$ ) purity by flow cytometry (Fig. 1B). Purity and identity of the respective cell populations were further confirmed by transcriptome analyses, showing the anticipated cell type specific transcript signatures for the $\mathrm{CM}$ and Fib populations (Fig. 1C). After casting of the EHM-reconstitution mixture, EHM undergo a first phase of matrix consolidation resulting in macroscopically visible compaction after 60 min (Tiburcy et al., 2014) and an on-set of rhythmic contractions within $72 \mathrm{~h}$ in culture; subsequent mechanical loading to support auxotonic contractions results in advanced tissue maturation (Tiburcy et al., 2017). This process is paralleled by the development of an anisotropic and wellstructured functional syncytium (Fig. 1D).

\subsection{Fibroblasts drive collagen type I hydrogel compaction}

In order to investigate the role of fibroblasts for EHM compaction, mechanical behavior and functional maturation, we generated collagen I hydrogels without cells, with either CMs or Fibs, or with both cell types (EHM). We quantified the compaction process after $24 \mathrm{~h}$ and $72 \mathrm{~h}$. In the presence of fibroblasts, collagen type I hydrogels were significantly more compacted than in the presence of either CMs or no cells at all (Fig. 2A and B). The compaction process was completed at $24 \mathrm{~h}$ for collagen type I hydrogels without cells, with CMs and with CMs plus Fibs. Collagen type I hydrogels populated with Fibs continued to compact (Fig. 2A and B). After 4 weeks, tissues were strikingly different as to their macroscopic appearance with a failure to compact in cell- and fibroblast-free models (Fig. 2B). Tissue compaction could further be quantified by the measurement of tissue thickness (i.e., the distance between the inner and outer tissue boundaries in $\mathrm{mm}$ : Collagen only $5.1 \pm 0.3 ;+\mathrm{CM} 4.8 \pm 0.2 ;+$ Fib $1.1 \pm 0.06 ;+\mathrm{CM} /+$ Fib $1.3 \pm 0.3$; $\mathrm{n}=12 / 9 / 10 / 12$; Fig. 2C). Staining with Sirius red confirmed that collagen fibers were compacted markedly in the fibroblasts containing tissue (Fig. 2D). Interestingly, we also noted some collagen fiber compaction in the CM only tissue, which could be supported by contaminating mesenchymal fibroblast-like cells ( $5 \%$ according to flow cytometry; Fig. 1B). The consequences of the failure to compact for contractile outcome could be confirmed by isometric force measurements with minimal twitch amplitudes in CM-only tissue versus maximal twitch amplitudes in the $\mathrm{CM}+$ Fib containing EHM (force of contraction [FOC] in $\mathrm{mN}$ at maximally effective extracellular calcium [ $4 \mathrm{mM}]: 0.2 \pm 0.05$ vs. $1.2 \pm 0.2$; ${ }^{*} \mathrm{p}<0.05$ by two-tailed unpaired Student's t-test; $\mathrm{n}=4$ /group; Fig. 2E).

\subsection{Mechanical properties of collagen network formation}

After the basic characterization of EHM consolidation and function from the first day to up to 4 weeks, we focused on the mechanical characterization of the applied collagen type I without a cellular component. Therefore, we first measured the temporal evolution of the shear storage $G^{\prime}$ and shear loss modulus $G^{\prime \prime}$ of pure collagen at $1 \%$ strain and a physiological frequency of $1 \mathrm{~Hz}$ to simulate the repetitive strain collagen would be exposed to in the human heart. The obtained data indicated that collagen stiffening by gelation is a material inherent process, which appears quickly after pH-neutralization and is completed after $30 \mathrm{~min}$ (Fig. 3). Average $G^{\prime}$ and $G^{\prime \prime}$ plateaued at $26.5 \pm 0.8 \mathrm{~Pa}$ and $4.1 \pm 0.3 \mathrm{~Pa}$, respectively.

\subsection{Differential contribution of fibroblasts and cardiomyocytes to collagen network consolidation}

Subsequent mechanical characterization of collagen type I hydrogels with and without cells (CMs and/or Fibs) confirmed the finding of a completion of the cell-independent collagen gelation 
A

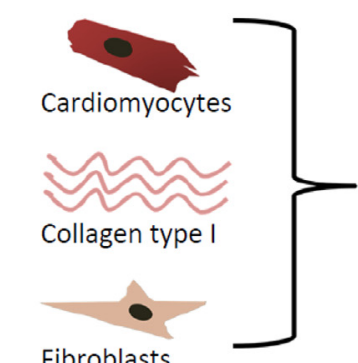

Fibroblasts

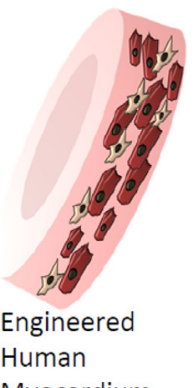

Myocardium

C
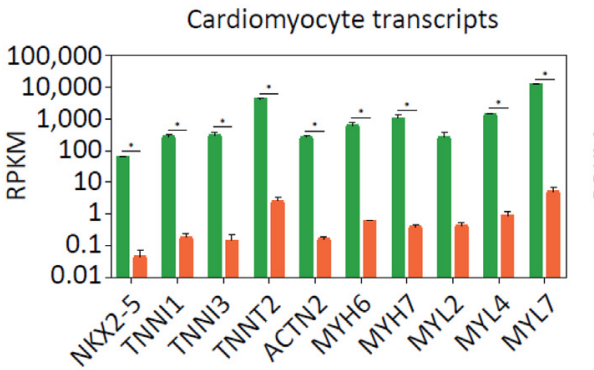

B
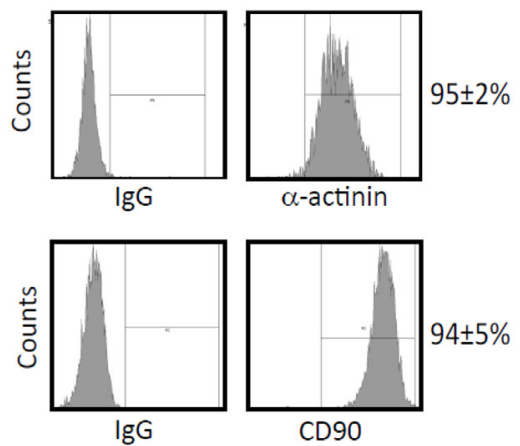

CD90

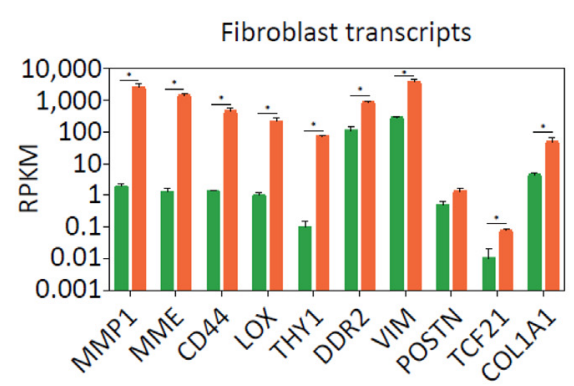

CM Fib

D

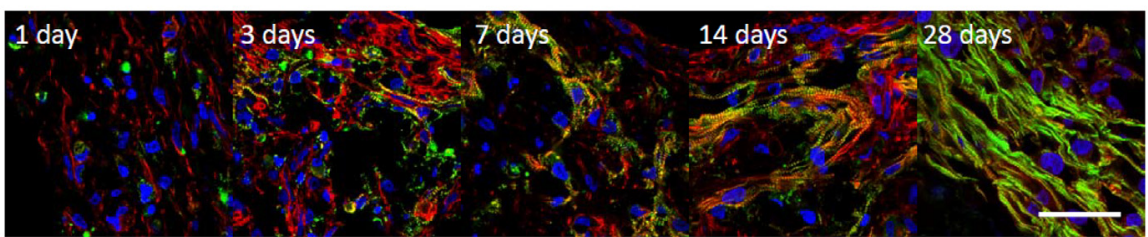

Fig. 1. Characterization of the engineered human myocardium (EHM) cell populations. (A) EHM were generated from human embryonic stem cell-derived cardiomyocytes (CMs) and human foreskin fibroblasts (Fibs) suspended in a collagen type I hydrogel (2:1 CM:Fib ratio). (B) Confirmation of input cell purity by flow cytometry for the CM marker "alphasarcomeric actinin" (ACTN2) and the Fib marker "CD90" (aka Thy-1). (C) Transcriptome analyses confirmed characteristic RNA profiles in CM and Fib populations (data $\mathrm{n}=3$ /group); CM and Fib transcript signatures were recently reported in detail in Tiburcy et al. (2017); RPKM: Reads Per Kilobase Million. *P < 0.05 CM vs. Fib by unpaired, two-tailed Student ttest. (D) Whole-mount EHM immune fluorescence imaging at the indicated days after casting; sarcomeric actinin (green) labels CMs, f-actin (red), nuclei (blue); scale bar: $50 \mu \mathrm{m}$ (all panels have the same magnification).

process within $30 \mathrm{~min}$ after $\mathrm{pH}$-neutralization (Fig. 4A and $\mathrm{B}$ ). Continuous collagen matrix stiffening was observed in collagen type I networks containing Fibs, with however a clear attenuation of the Fib-mediated stiffening process in the presence of CMs (resembling the EHM mixture). These findings were in line with the previously reported macroscopically visible compaction of EHM as early as $60 \mathrm{~min}$ after casting (Tiburcy et al., 2014) as well as the in this study documented continuous tissue compaction process (Fig. 2A-D). The associated changes in viscoelastic properties could be determined quantitatively by cone and plate shear rheology as changes of $G^{\prime}$ and $G^{\prime \prime}$ as a function of time after the cellindependent collagen gelation was completed, i.e., 30-60 min after the start of the experiment (Fig. $4 \mathrm{~A}-\mathrm{C}$ ). Collectively, these data identify distinct phases of cell-independent gelation $(0-30 \mathrm{~min})$ and largely Fib-dependent (in Fib and EHM samples) tissue compaction ( $>30 \mathrm{~min}$ ), which appears to be modulated by cardiomyocytes in the EHM samples (Fig. 4D).

\subsection{Differential Fib and CM transcriptomes during early EHM compaction}

To interrogate potential molecular changes during fibroblastdependent EHM compaction, we performed differential transcriptome analyses by RNA-sequencing. By comparing the cell transcriptomes at baseline, i.e., after a 30 min cell seeding and largely cell-independent collagen gelation phase without obvious differences in cell-induced viscoelastic properties, and after additional $60 \mathrm{~min}$ in which we observed either no further (in CM populated collagen) or Fib-mediated collagen hydrogel stiffening (Fig. 5A). As anticipated, a principle component analysis (PCA; Fig. 5B) of the RNA-sequencing data reflected for the most part ( $91 \%$ in principle component [PC] 1) the differences in cell composition: (1) CM: only cardiomyocytes; (2) Fib: only fibroblasts; (3) EHM: CM:Fib at a 2:1 input ratio. PC2 (7\%) separated the single cell type hydrogel cultures from the mixed cultures comprising the same cells, suggesting a transcriptionally effective $\mathrm{CM}$ :Fib cross-talk. The transcriptional changes within the time-frame of $60 \mathrm{~min}$ of no (CM group) or Fib-mediated (Fib and EHM groups) collagen compaction were small as anticipated (Fig. 5B). It was however notable that Fibs in comparison to CMs presented a markedly enhanced differential transcription within the 60 min observation period (DEG; 1,379 vs. 369; Fig. 5C). From those, 202 DEGs were commonly regulated in all investigated groups and according to GO term analyses associated with "general tissue formation processes" (Supplemental Table 1). The Fib-specific expression profile (1,161 DEGs; Supplemental Table 1) included evidence for markedly enhanced transcriptional 


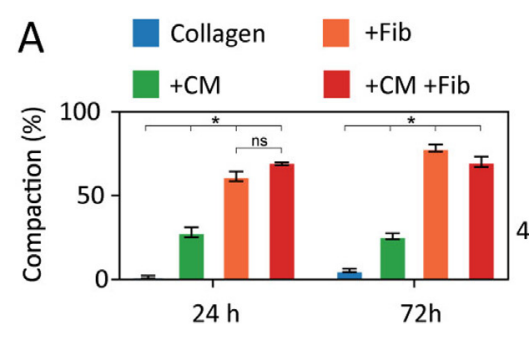

C

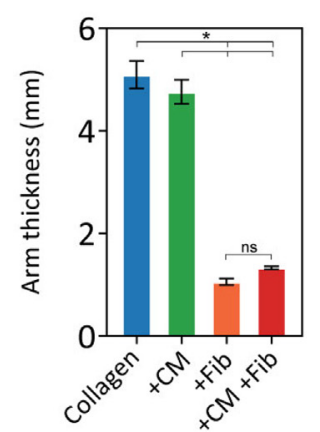

B

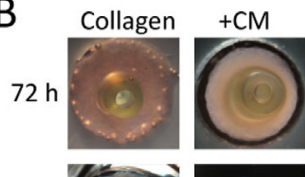
4 weeks

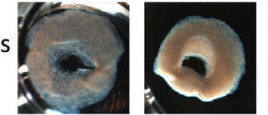

$\mathrm{E}$

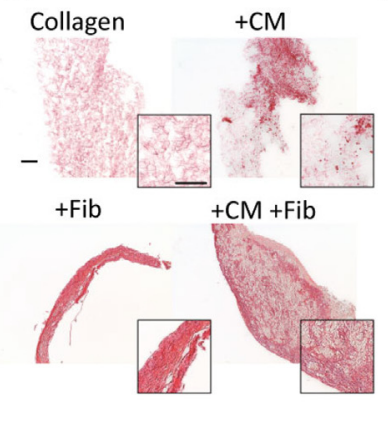

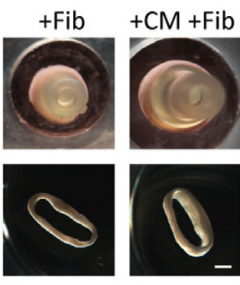

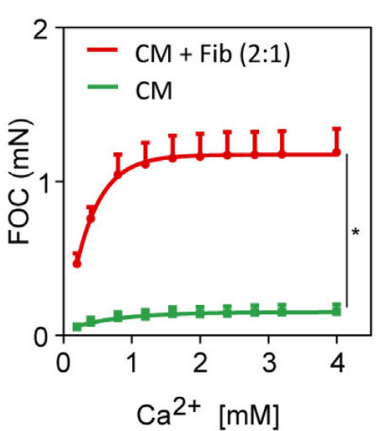

Fig. 2. Fibroblast controlled tissue compaction. (A) Collagen type I hydrogels were generated containing either no cells or Fibs ( 430,000/tissue) and/or CMs ( 860,000/tissue) Compaction was assessed $24 \mathrm{~h}$ and $72 \mathrm{~h}$ post-casting into circular PDMS molds with a central Teflon rod. *p < 0.05 vs. Collagen by 1 -way ANOVA followed by Sidak multiple comparison test. (B) Representative images of compacted tissue at $72 \mathrm{~h}$ (within the casting molds) and 4 weeks of culture (free floating after release from the casting molds). Scale bar: $1 \mathrm{~mm}$ (applies to all panels). (C) EHM combined arm thickness at 4 weeks in culture; ${ }^{*} \mathrm{p}<0.05$ vs. Collagen or + CM as indicated by 1 -way ANOVA followed by Tukey multiple comparison test, $\mathrm{n}=9-12$ /group. (D) Sirius red tissue sections to highlight fibrillary collagen at 4 weeks in culture. Scale bars: $100 \mu \mathrm{m}$ (overviews) and $400 \mu \mathrm{m}$ (inset). (E) Force of contraction (FOC) of EHM (CM + Fib) and CM-only containing tissue after 4 weeks in culture; FOC was measured at $37^{\circ} \mathrm{C}$ in Tyrode's solution under electric pulsed-field stimulation at $1.5 \mathrm{~Hz}$; the characteristic increase in FOC as a function of increasing extracellular calcium is displayed. *p $<0.05$ by 2 -way ANOVA; $n=4 /$ group.
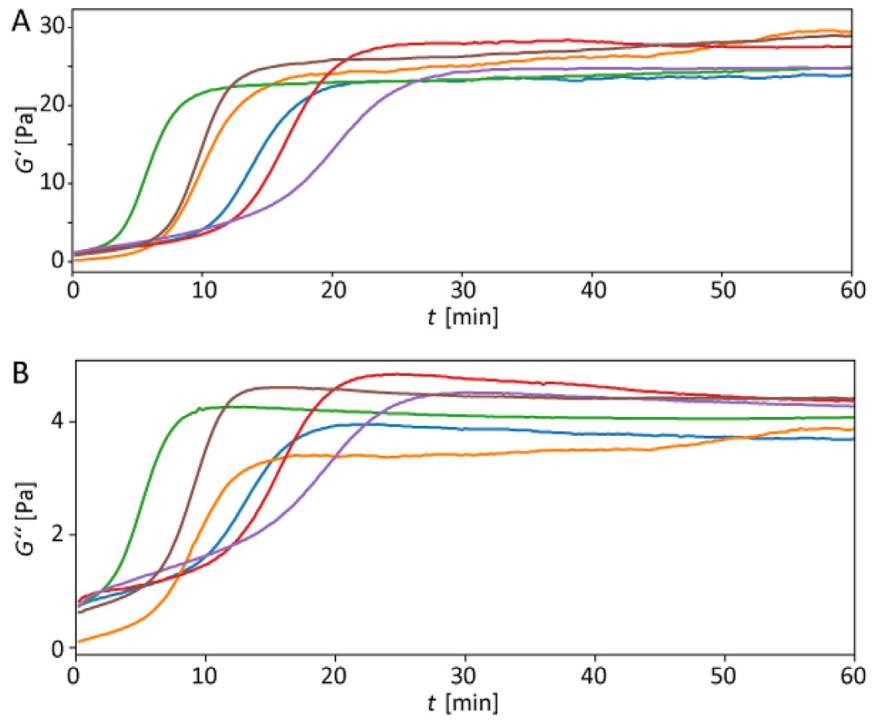

Fig. 3. Mechanical characterization of collagen type-I hydrogel gelation. Storage modulus G' (A) and loss modulus G' (B) plotted as a function of time. Individual $(\mathrm{n}=6)$ rheology measurements of cell-free collagen type I hydrogels were performed using a cone $\left(2^{\circ}\right)$ and plate bulk rheometer. Gelation of acidic acid solubilized collagen type I was started by $\mathrm{pH}$-neutralization with $\mathrm{NaOH}$ at room temperature $\left(23^{\circ} \mathrm{C}\right)$.

activity and processes related to "heart development" and "extracellular matrix organization", with for example an upregulation of SOX9, described as a master regulator of organ development and collagen expression (Hanley et al., 2008; Lacraz et al., 2017); this finding was in line with our earlier observation of a strong contribution of endogenously produced collagen type I in EHM formation (Tiburcy et al., 2011). The cardiomyocyte-specific 154 DEGs
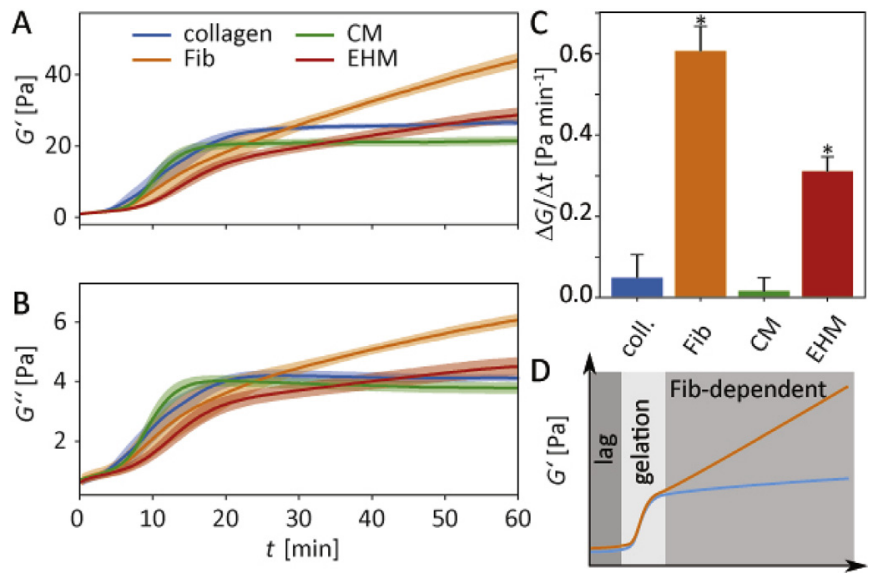

Fig. 4. Biophysical properties of consolidating cell-free and cell-containing collagen type I hydrogels. Samples of collagen type I containing no cells (Collagen; $n=6$ ), CMs $(n=3)$, Fibs $(n=4)$, and both cell types (EHM; $n=4)$ were mechanically analyzed using a cone and plate rheometer. Applying fixed $1 \%$ strain at $1 \mathrm{~Hz}$ the storage modulus $G^{\prime}(A)$ and loss modulus G" (B) were recorded over a timeframe of $60 \mathrm{~min}$; extended measurements were technically difficult to perform due to the drying of the hydrogel formulation. (C) Average change of $\mathrm{G}^{\prime}$ as a function of time $\left(\Delta \mathrm{G}^{\prime} / \Delta \mathrm{t}\right.$ in $\left.\mathrm{Pa} \mathrm{min}^{-1}\right)$ during the 30-60 min (cell-dependent compaction) interval. ${ }^{*} \mathrm{p}<0.001 \mathrm{vs}$. Collagen (coll.) by 1-way ANOVA and Tukey's multiple comparison. (D) Schematic summary describing the distinct phases of cell-independent collagen hydrogel gelation and Fib-dependent (in Fib and EHM groups) collagen hydrogel compaction.

indicated enhanced endoplasmic reticulum (ER)-stress (Supplemental Table 1), which may trigger apoptotic processes. Interestingly, transcriptional signatures for the activation of "apoptotic processes" were markedly enhanced in the presence of Fibs and Fib-dependent matrix stiffening. This finding is in agreement with the observed over-proportional loss of cardiomyocytes 

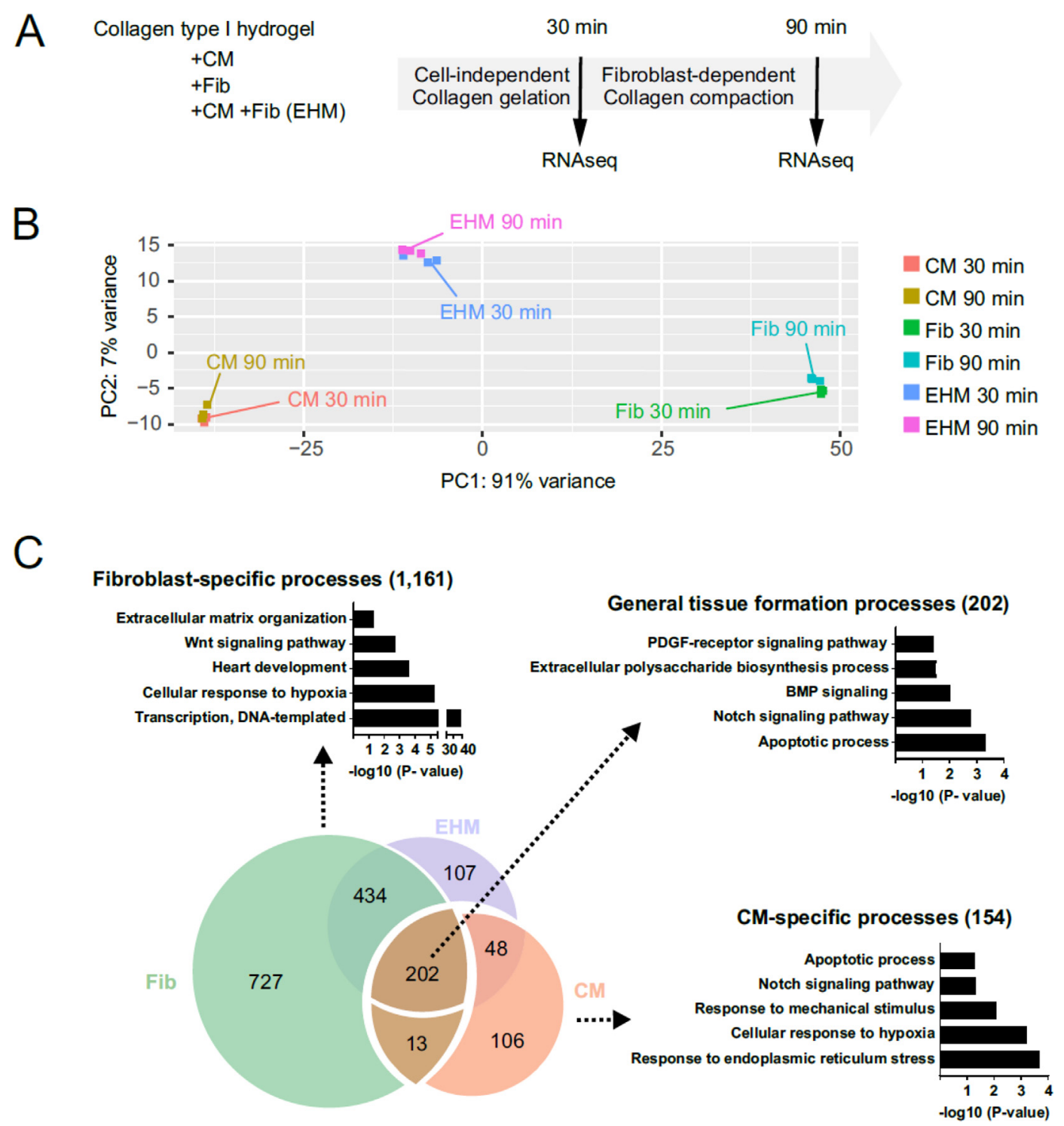

Fig. 5. Differential transcriptomes in Fibs and CM in 3D hydrogel cultures. (A) Schematic overview of the experimental design ( $\mathrm{n}=3$ samples per group). (B) PCA plot of global gene expression of the respective hydrogel samples at the two investigated time-points (1: baseline after completion of cell-independent collagen gelation (30 min); 2 : after additional 60 min of primarily Fib-mediated collagen compaction (90 min). (C) Venn diagram comprising the identified differentially expressed genes (DEGs) assigned to specific CM, Fib and general cell type-independent processes according to GO term analyses; all DEGs are listed in Supplementary Table 1).

especially during the early EHM formation process (Tiburcy et al., 2011). Cell loss in EHM is a combined result of irreversible damage by the enzymatic cell isolation procedure needed for EHM preparation, anoikis, and apoptosis; leading collectively to a notable change in CM:Fib composition from 2:1 (input) to $1: 1$ (output) during culture (Tiburcy et al., 2017). A further detailed analysis of the DEGs underlying PC2 (CM:Fib cross-talk in EHM vs the single cell culture formats) indicated 45 up- and 405 downregulated transcripts; Supplementary Table 2), comprising a large content of non-coding RNA (210 of in total 450 transcripts, including miR and lncRNA).

\subsection{Nonlinear elastic behavior at physiologically relevant strains}

The rheological data reported so far were obtained at a fixed $1 \%$ strain to enable studies of the linear stress-strain response over an extended time period $(60 \mathrm{~min})$ under well controlled conditions (Figs. 3 and 4) without altering the collagen gelation processes.
Considering that biopolymers and tissues exhibit a nonlinear strain-stiffening viscoelastic response (Storm et al., 2005) and that cardiomyocytes and cardiac muscle function at larger strains ( $\gamma_{0}$ up to approx. 20\% (Chuang et al., 2010; Hersch et al., 2013; Kajzar et al., 2008)), we performed large amplitude oscillatory shear (LAOS) measurements according to a previously reported method (Ewoldt et al., 2008) 60 min after triggering matrix consolidation by $\mathrm{pH}$ neutralization. LAOS allowed us to measure the stress-strain relation for one cycle as a function of time (Fig. 6A), followed by repeated measurements of the same sample at increasing strains. Plotting stress $\sigma$ vs strain $\gamma$ (Lissajous plot) allows for a visualization and robust quantification of the nonlinear strain-stiffening behavior (Fig. 6B). We first validated the LAOS measurements for different maximal strains $\left(\gamma_{0}=1 \%, 2 \%, 4 \%, 7 \%, 8 \%\right)$ on pure collagen type I networks and confirmed previous observation of strainstiffening, which became obvious at strains $\gamma_{0}>2 \%$ (Fig. 6C). We further analyzed the stress response by a Fourier analysis of the stress signal (Ewoldt et al., 2008). For reasons of symmetry, only the 

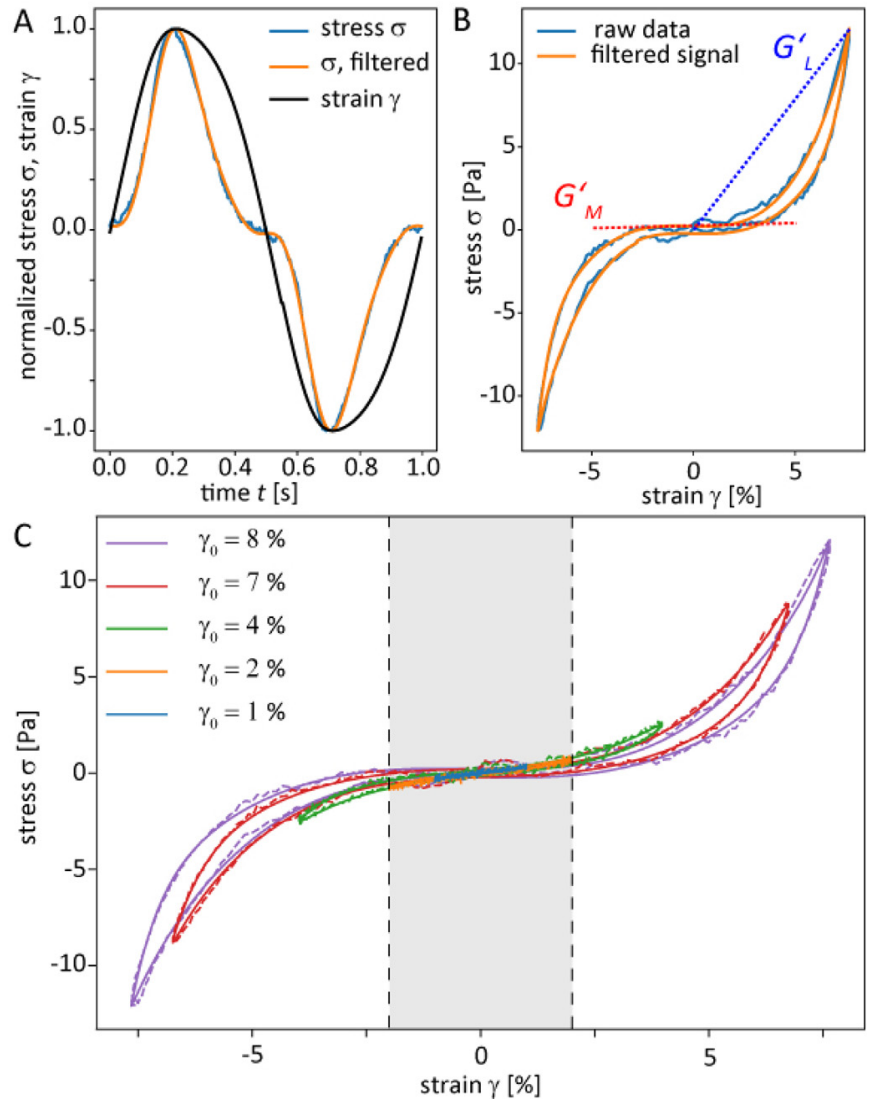

Fig. 6. Large amplitude oscillatory shear (LAOS) measurements of $\mathrm{pH}$ neutralized collagen hydrogels. (A) Representative data from one measurement cycle with normalized stress $\sigma$ (blue) and filtered stress $\sigma$ (orange) at a defined strain $(\gamma)$ amplitude (black- y-axis) as a function of time (x-axis). (B) Lissajous plot transformation of the data displayed in A revealed the anticipated nonlinear stress-strain response for collagen type I networks. The raw stress signal (blue) was filtered (orange) by only using the first three non-zero, odd Fourier coefficients, i.e., the first, third and fifth Fourier mode. Indicated with dashed lines are the large-strain modulus $G_{L}$ (blue) and the minimum-strain modulus $G_{M}^{\prime}$ (red). (C) Lissajous plots for collagen exposed to the indicated increasing strain $\left(\gamma_{0}\right)$ amplitudes. The raw signals are depicted as dashed lines; the filtered signals are displayed as solid lines; the different strain amplitudes are indicated by different colors. For strains $\gamma_{0}=1 \%$ and $2 \%$ (range indicated in light grey between two vertical dashed lines) the measurement showed a linear relation between stress and strain, but for larger strains $\left(\gamma_{0}>2 \%\right)$ nonlinear strain-stiffening became evident.

first three non-zero odd Fourier coefficients, i.e., the first, third and fifth Fourier mode, were used and found sufficient to match the measured data (Fig. 6; measured stress data in blue vs. filtered stress data in orange). Therefore, the stress-strain behavior of collagen can be described in the following form:

$\sigma(\gamma, \dot{\gamma})=\sum_{n \in\{1,3,5\}}\left[\gamma_{0} e_{n} T_{n}\left(\frac{\gamma}{\gamma_{0}}\right)+\gamma_{0} v_{n} T_{n}\left(\frac{\dot{\gamma}}{\dot{\gamma}_{0}}\right)\right]$

$T_{n}(x)$ is the $n$-th Chebyshev polynomial, $e_{n}$ and $v_{n}$ are obtained from a Fourier transformation. Since this relation requires a strictly sinusoidal strain input, the stress response simply is a fifth-order polynomial in strain and strain rate.

In order to quantify the non-linearity of the strain response of the different samples of collagen with and without cells, we use the strain-stiffening ratio $S$ where $G_{L}^{\prime}$ denotes the large-strain modulus (dashed blue line in Fig. 6B) or secant modulus at maximal strain $\gamma=\gamma_{0}, G_{M}^{\prime}$ (dashed red line in Fig. 6B) is the minimum-strain modulus or tangential modulus at $\gamma=0$. A strain-stiffening ratio
$S \approx 0$ indicates a linear stress-strain relation, $S>0$ is a sign of strain stiffening (Ewoldt et al., 2008). A direct comparison of cell-free and cell-containing collagen hydrogels (Fig. 7) indicated similar strainstiffening with however the largest $S$ in the cell-free collagen formulation: $S \quad\left(\gamma_{0}=8 \%\right)=0.849 \pm 0.017 \quad$ (collagen, $\mathrm{n}=6$ ), $0.710 \pm 0.039(\mathrm{CM}, \mathrm{n}=3), \quad 0.747 \pm 0.027 \quad(\mathrm{Fib}, \mathrm{n}=4)$, and $0.675 \pm 0.037$ ( $E H M, n=3$ ). Collagen thus not only displays the largest stress at maximum strain $(\sigma=8.78 \pm 0.92 \mathrm{~Pa})$, but also the most nonlinear response. Cells in general reduced strain stiffening and thus effectively softened the collagen type I hydrogels. In line with their prominent role in collagen type I hydrogel compaction (Fig. 4), Fib-populated collagen type I hydrogels exhibited a slightly higher strain-stiffening and maximal stress than CMs or CM:Fib (EHM) mixtures.

\subsection{Mechanical ad-hoc model}

In addition to using a Fourier analysis to obtain an expression for the stress response as a function of strain and strain rate, we established an ad-hoc model for the time dependence of the stress response that reproduces the measured stress when driven with the corresponding sinusoidal strain input. The aim was to find an expression of the form

$\frac{d \sigma}{d t}=f\left(\sigma, \gamma, \dot{\gamma} ;\left\{c_{i}\right\}\right)$

such that the time series $\sigma(t)$ obtained by integrating the above differential equation matches the measured stress data. $\left\{c_{i}\right\}$ are model parameters that can be found by minimizing the quadratic differences between the stress obtained from the model and the measured stress. The resulting model not only reproduces the measured data, but also is applicable for different than sinusoidal strain input. This is in particular important for future modelling of the more complex function of strain during a physiological contraction cycle of (engineered) human myocardium. As candidate for $f$ we used a third order polynomial in $\sigma, \gamma$, and $\dot{\gamma}$. For reasons of symmetry, we considered only odd terms. During optimization we found that further terms could be omitted leaving
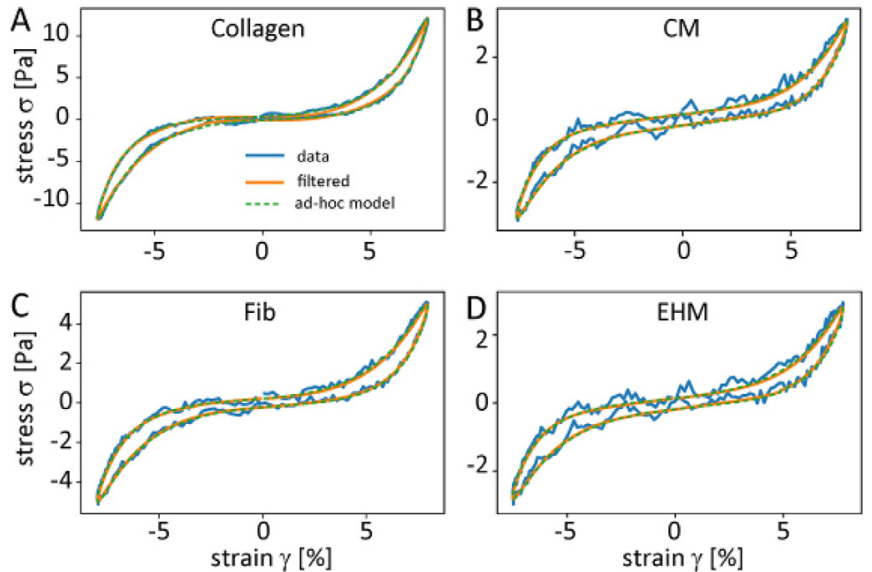

Fig. 7. Lissajous plots to assess strain-stress behavior in cell-free and cell-containing collagen type I hydrogels. All samples showed characteristic strain-stiffening. Cellfree collagen type I networks demonstrated the largest stress at maximum strain $\left(\sigma=8.78 \pm 0.92\right.$ Pa at $\left.\gamma_{0}=8 \% ; n=6\right)$. There was a trend (not significant) towards higher strain-stiffening $\left(\sigma=4.40 \pm 0.44 \mathrm{~Pa}\right.$ at $\left.\gamma_{0}=8 \% ; \mathrm{n}=4\right)$ by Fibs than CMs $\left(\sigma=3.77 \pm 0.44 \mathrm{~Pa}\right.$ at $\gamma_{0}=8 \% ; \mathrm{n}=3$ ) or CM:Fib (EHM; $\sigma=2.68 \pm 0.15 \mathrm{~Pa}$ at $\gamma_{0}=8 \%$; $\mathrm{n}=3$ ). The graphs include data from the ad-hoc model (dashed green lines) demonstrating that the observed biological phenomena can be modelled mathematically. 


$$
\begin{aligned}
f(\sigma, \gamma, \dot{\gamma})= & c_{1} \gamma+c_{2} \dot{\gamma}+c_{3} \gamma^{2} \dot{\gamma}+c_{4} \gamma^{2} \sigma+c_{5} \gamma \dot{\gamma}^{2}+c_{6} \gamma \dot{\gamma} \sigma+c_{7} \gamma \sigma^{2} \\
& +c_{8} \dot{\gamma}^{3}+c_{9} \sigma^{3}
\end{aligned}
$$

as candidate. Optimization of the model parameters was performed using a basin-hopping algorithm (Wales and Doye, 1997). After optimization, this ad-hoc model was able to recover the measured stress data (Fig. 7). The model parameters used to obtain the curves for the four samples are listed in Supplemental Table 3.

\section{Discussion}

Engineering of heart muscle requires a profound understanding of cellular cross-talk and tissue biomechanics. The role of fibroblasts or fibroblast-like cells for the formation of EHM (Tiburcy et al., 2017) and similar tissue engineering approaches has been clearly recognized (Kensah et al., 2013; Naito et al., 2006; Soong et al., 2012; Zhang et al., 2013). The influence of fibroblasts on the in vitro heart muscle formation process becomes very obvious already within minutes to hours after EHM reconstitution, namely by its failure to compact, in the absence of fibroblasts. Here, we investigated the EHM consolidation process with a particular focus on determining the differential impact of fibroblasts and cardiomyocytes. Our data identify that the process of EHM consolidation comprises a cell-independent collagen gelation phase, followed by a fibroblast-mediated collagen compaction phase, which is likely mediated by direct fibroblast-collagen interactions via for example integrins (Herum et al., 2017). In the presence of cardiomyocytes, Fib-mediated collagen type I network compaction was clearly attenuated, suggesting a biomechanically relevant Fib:CM cross-talk. Together with the finding that environmental viscoelastic stiffening enhances extracellular matrix synthesis by Fibs and despite studying "only" engineered human heart muscle, it may be prudent to speculate that our data suggests a fibrosis control function by cardiomyocytes. The contribution of cardiomyocyte:fibroblast cross-talk to tissue fibrosis has been investigated intensively, with clear evidence for the contribution of noncoding RNA in addition to paracrine mechanisms via secreted growth factors (reviewed in (Beermann et al., 2016; Viereck et al., 2014)). The identification of a numerical model to describe the biomechanical properties, including nonlinear strain-stiffening at physiological strains, of cell-free and cell-populated collagens will not only be important for the further optimization of EHM, but may also foster our understanding of the fundamental principles underlying cell:matrix interactions for heart function in general.

We applied neonatal human foreskin fibroblasts (HFFs) rather than organ specific fibroblasts in this study for practical (homogenous, well-defined cell model) and biological reasons (similar supportive function was observed in EHM from HFF and fibroblasts of other sources, including heart; unpublished observation). Earlier data suggested that fibroblasts provide important cues for the formation of EHM, which includes the secretion and build-up of extracellular matrix (Tiburcy et al., 2011). The data obtained in this study extends our previous findings by demonstrating a transcriptional activation, which may contribute to the subsequent and continuous extracellular matrix formation processes in EHM. This appears to involve for example enhanced expression of hyaluronan synthases [HAS1 and HAS2], PDGFA, and BMP4 (common DEGs) as well as ELN (elastin), PDGFB, TGFB1, and SOX9 (Fib-specific DEGs) all DEGs can be obtained from the Supplemental Table 1. The observed evidence for ER-stress in the cardiomyocyte pool, which may lead to the activation of apoptotic processes, is well in line with the observed enhanced caspase activity during the early EHM formation process (Tiburcy et al., 2011). Several means have been developed to counter cardiomyocytes loss in EHM, including the application of cardio-protective molecules such as insulin/IGF-1 (Naito et al., 2006) and PDGF-BB (Vantler et al., 2010). The obtained evidence of non-coding RNA mediated cross-talk between fibroblasts and cardiomyocytes in EHM (Supplemental Table 2) was a notable observation and will require further detailed studies, including studies on exosome/microvesicle mediated cross-talk, a mechanisms described to in tumor environment stiffening (Bonnans et al., 2014; Villasante et al., 2016), and analyses of proteome alterations by the observed non-coding RNA as well as the associated consequences for the long-term regulation of ECM viscoelasticity.

A particular goal of this study was to go beyond the description of biological processes and potentially involved mechanisms and establish the basis for the formulation of mechanical models of the EHM formation process. For this, viscoelastic properties of collagen type I and its alterations in the presence of the key cellular components of EHM, i.e., cardiomyocytes and fibroblasts, must be provided. By in situ rheological measurements we collected quantitative data of $G^{\prime}$ and $G^{\prime \prime}$ during early cell-independent and cell/ Fib-dependent tissue compaction as well as important insight into the nonlinear strain stiffening properties of collagen without or populated with cells. An interesting observation was the cellinduced softening of collagen at high strains. This may be explained by the soft mechanical properties of cells per se and is in line with studies on the biomechanics of multi-component tissues (Huber et al., 2013; Marquez et al., 2010). Activation of cell intrinsic and mainly fibroblast-associated mechanisms appears to counter the softening process, likely by additional extracellular matrix synthesis and mechanical pre-straining of the extracellular matrix into the nonlinear strain-stiffening range. In essence, fibroblasts and cardiomyocytes in collagen type I hydrogels appear to selforganize the EHM formation process by a dynamic adaptation of the viscoelastic properties of the surrounding extracellular matrix environment. Finally, the introduced numerical ad-hoc model is a first step towards future simulations of the viscoelastic properties of EHM (Stein et al., 2017), in particular with respect to the more complex stress-strain relation during a contraction cycle. In combination with novel models of cross-linked and anisotropic networks (Heidemann et al., 2015, 2018) this may allow for the identification of ideal cell compositions and externally imposed stress-strain behavior, either by mechanical means (Liaw and Zimmermann, 2016) or electrical stimulation (Godier-Furnemont et al., 2015) for optimal tissue development.

\section{Materials and methods}

\subsection{Cell culture}

Human neonatal foreskin fibroblasts (Fibs) were obtained from ATCC (HFF-1, SCRC-1041) and maintained in DMEM containing $4 \mathrm{mM}$ L-glutamine, $4,500 \mathrm{mg} / \mathrm{L}$ glucose, $1 \mathrm{mM}$ sodium pyruvate, and $1,500 \mathrm{mg} / \mathrm{L}$ sodium bicarbonate supplemented with $15 \%$ fetal calf serum (FCS), $100 \mathrm{U} / \mathrm{mL}$ Penicillin, and $100 \mu \mathrm{g} / \mathrm{mL}$ Streptomycin $(\mathrm{P} / \mathrm{S}$; Thermo Scientific). Cell counting was done with a CASY TTC system (Roche) using the electric current exclusion method. Fibs were used for experiments from passage 18-22. Cardiomyocytes were derived from HES2 (ES International, Singapore) or a genetically modified derivative HES2-RFP (Irion et al., 2007). Ethical approval for the use of human embryonic stem cells (ESCs) was obtained from the Central Ethic Committee for Stem cell Research (ZES; permit \#12; reference number: 1710-79-1-4-16). ESCs were grown and differentiated as reported previously (Tiburcy et al., 2017). Cardiomyocyte purity was analyzed by flow cytometry (Fig. 1B). 


\subsection{Flow cytometry}

Single cardiomyocyte cell suspensions were prepared by digesting cardiomyocyte monolayers with a mixture of Accutase (Millipore), $0.0125 \%$ Trypsin (Thermo Scientific), and $20 \mu \mathrm{g} / \mathrm{ml}$ DNAse (Calbiochem) for $15-30 \mathrm{~min}$ at room temperature. Fibroblasts were prepared by digestion with TrypLE (Thermo Sientific) for $10 \mathrm{~min}$ at $37^{\circ} \mathrm{C}$. Cells were resuspended in culture medium, centrifuged at $300 \mathrm{~g}$ for $5 \mathrm{~min}$, and incubated with 1:1,000 Sytox Red Dead Cell Stain ( $5 \mu \mathrm{mol} / \mathrm{L}$ stock solution; Thermo Scientific) and Brilliant Stain Buffer containing mouse anti-human BUV395conjugated CD90 IgG1, $\mathrm{K}$ (BD Biosciences, clone 5E10, $5 \mu \mathrm{l}$ per sample) for $20 \mathrm{~min}$ at $4{ }^{\circ} \mathrm{C}$. CM suspensions were centrifuged at $300 \mathrm{~g}$ for $5 \mathrm{~min}$ and then fixed in $70 \%$ ice-cold ethanol. After fixation, cells were centrifuged once more and resuspended in blocking buffer (PBS containing $1 \mathrm{mg} / \mathrm{ml}$ BSA [Sigma], 5\% FCS [Thermo Fisher] and $0.1 \%$ Triton 100X [Sigma]). After 10 min of blocking, cells were pelleted by centrifugation and resuspended in blocking buffer with primary antibody (sarcomeric $\alpha$-actinin 1:4,000 SigmaAldrich A7811, mouse-monoclonal) or respective IgG1 isotype control for $45 \mathrm{~min}$ at $4{ }^{\circ} \mathrm{C}$. Cells were washed twice with PBS, followed by a washing step in blocking buffer and subsequent incubation in secondary antibody (1:1,000 anti-mouse 488 [A-11001] or 633 [A-21052], Thermo Fisher) and Hoechst $(10 \mathrm{ng} / \mathrm{ml}$; Thermo Fisher) for $30 \mathrm{~min}$ at $4{ }^{\circ} \mathrm{C}$. Cells were washed with PBS and finally resuspended in PBS for analysis. The gating strategy was described in detail in (Tiburcy et al., 2017). 10,000 live cell events were analyzed per sample. Measurements were performed on a LSRII SORP Cytometer and analyzed using the DIVA software (BD Biosciences). FACS visual plots were generated using Flowing Software 2.5.1. (Perttu Terho, University of Turku, Finland).

\subsection{Hydrogel culture preparations and analysis}

Two batches of medical-grade bovine collagen (LLC Collagen Solutions) were used in this study (Batch 1:15CSA02; Batch 2: 17CSA03). Equal volumes of collagen $(0.9 \mathrm{mg} / \mathrm{ml})$ and concentrated serum-free medium (2x RPMI, 8\% B27 without insulin, $200 \mathrm{U} / \mathrm{ml}$ penicillin, and $200 \mu \mathrm{g} / \mathrm{ml}$ streptomycin) were mixed on ice. Cells $(950,000 \mathrm{Fibs} / \mathrm{ml}, 1,900,000 \mathrm{CMs} / \mathrm{ml}$, or both cell types at a $1: 2$ ratio - 2,850,000 cells/ml) were added after $\mathrm{pH}$ neutralization by dropwise addition of $0.1 \mathrm{~N} \mathrm{NaOH}$. Single cell suspensions were prepared as described above for the flow cytometry experiments. Cells were suspended in EHM culture medium: Iscove's medium with 4\% B27 without insulin, $1 \%$ non-essential amino acids, $2 \mathrm{mmol} / \mathrm{l}$ glutamine, $300 \mu \mathrm{mol} / \mathrm{l}$ ascorbic acid, $100 \mathrm{ng} / \mathrm{ml}$ IGF1 (AF-100-11), $10 \mathrm{ng} / \mathrm{ml}$ FGF-2 (AF-100-18B), $5 \mathrm{ng} / \mathrm{ml} \mathrm{VEGF}_{165}$ (AF-100-20), $5 \mathrm{ng} / \mathrm{ml}$ TGF- $\beta 1$ (AF-100-21C), and $\mathrm{P} / \mathrm{S}$ (all growth factors were obtained from PeproTech). An equal amount of EHM culture medium without cells was added in the cell free, collagen only, conditions. 149 and $450 \mu \mathrm{l}$ hydrogel:cell mixtures were used for to rheology measurements or cast into circular models to generate tissue loops (Soong et al., 2012; Tiburcy et al., 2017). Hydrogel consolidation was studied for $60 \mathrm{~min}$ in a bulk rheometer or after 24 and $72 \mathrm{~h}$ in the circular casting molds. The size of compacted hydrogels and total arm thickness of 4-week-old tissues was determined using a Lumar system equipped with an AxioCam MRc and Axiovert 4.1 software (Zeiss). For the latter analyses 10-20 individual measurements of tissue thickness per tissue were averaged.

\subsection{Histology}

For whole-mount analyses, hydrogels/EHM were fixed in $4 \%$ formaldehyde (Histofix, Roth) for at least $2 \mathrm{~h}$ at room temperature. After washing for $4 \mathrm{~h}$ at room temperature (RT) in blocking buffer
(PBS containing $1 \mathrm{mg} / \mathrm{ml}$ bovine serum albumin [Sigma], 5\% FCS [Gibco], and $0.1 \%$ Triton [Sigma]) the primary antibodies were added for $48 \mathrm{~h}$ at $4{ }^{\circ} \mathrm{C}$. Following three washes with PBS for 60 min at RT the secondary antibodies, together with phalloidin (Thermo Scientific), were added for $48 \mathrm{~h}$ at $4{ }^{\circ} \mathrm{C}$. After three more washes, tissues were mounted with Fluoromount (SouthernBiotech) and imaged using a Zeiss confocal microscope (CLSM; Zeiss 710 LSM/NLO). For cryo-sectioning, tissues were immersed in $30 \%$ sucrose overnight at $4{ }^{\circ} \mathrm{C}$ and embedded the following day in OCT (TissueTech) by slow freezing on a liquid nitrogen-cooled metal block. Tissues were stored at $-20^{\circ} \mathrm{C}$ and cut at $10 \mu \mathrm{m}$ with a cryotome (Leica). Tissues were dried at RT before blocking for $30 \mathrm{~min}$ and treated with primary antibodies (or respective isotype controls) overnight at $4{ }^{\circ} \mathrm{C}$, followed by three washes with PBS for 20 min and incubation with secondary antibody and Hoechst for $2 \mathrm{~h}$ at RT. After three final washes, tissue mounting was with Fluoromount (SouthernBiotech). Sections were imaged using a Zeiss confocal microscope (CLSM; Zeiss 710 LSM/NLO). For Sirius Red staining, tissues were embedded in paraffin (Roth). $4 \mu \mathrm{m}$ sections were cut, dewaxed, and rehydrated using standard protocols. Nuclei were stained with Mayer's Hemalaun solution (AppliChem) for 5 min. After two washes with $\mathrm{H}_{2} \mathrm{O}$ slides were incubated with Picrosirius red solution for at least $1 \mathrm{~h}$. Subsequently, the slides were washed twice with acetic acid $\left(5 \% \mathrm{v} / \mathrm{v}\right.$ in $\left.\mathrm{H}_{2} \mathrm{O}\right)$ for $5 \mathrm{~min}$, followed by clearing in xylol (Roth). Slides were mounted using Histokitt II (Roti). Slides were imaged using a Zeiss Axiovert microscope and analyzed using ZEN software.

\subsection{Contraction analysis}

Tissue constructed in a loop format, were isometrically suspended in organ baths (Föhr Medical Instruments) filled with Tyrode's solution (in mmol/L: $120 \mathrm{NaCl}, 1 \mathrm{MgCl} 2,0.2 \mathrm{CaCl} 2,5.4 \mathrm{KCl}$, $22.6 \mathrm{NaHCO}, 4.2 \mathrm{NaH} 2 \mathrm{PO} 4,5.6$ glucose, and 0.56 ascorbate) at $37{ }^{\circ} \mathrm{C}$ and constant bubbling with $5 \% \mathrm{CO}_{2}$ and $95 \% \mathrm{O}_{2}$. Force of contraction measurements were performed at $1.5 \mathrm{~Hz}$ electrical field stimulation with $5 \mathrm{~ms}$ square pulses $(200 \mathrm{~mA})$ and calcium concentrations increasing from 0.2 to $4 \mathrm{mmol} / \mathrm{L}$ as described previously (Tiburcy et al., 2017).

\subsection{Rheometry}

Mechanical properties of the hydrogel samples with and without cells were investigated with a bulk rheometer (MCR-501, Anton Paar, Austria) using a cone and plate geometry with an angle of $2^{\circ}$ and a gap height of $103 \mu \mathrm{m}$. The temporal evolution of the mechanical properties were monitored with measurements every $30 \mathrm{~s}$ using a sinusoidal strain of $1 \%$ at $1 \mathrm{~Hz}$. This low strain ensures to be in the linear response regime and does not alter the gelation process (data not shown). Dehydration of the sample was avoided by keeping the environment in a custom-made measurement chamber saturated with water vapor. The temperature was kept constant at $23^{\circ} \mathrm{C}$. For the nonlinear analysis the built-in LAOS routines of the rheometer software were used. The general setup was the same as described above and LAOS measurements were performed directly after finishing the measurement of the temporal evolution (on the same sample). While the frequency of the strain input remained at $1 \mathrm{~Hz}$ the strain amplitude was increased from $\gamma_{0}=1 \%$ to $\gamma_{0}=10 \%$ in steps of $1 \%$. The stress response over one period of oscillation (i.e. $1 \mathrm{~s}$ ) was recorded and stress data were sampled with 256 points per period (every $3.9 \mathrm{~ms}$ ).

\subsection{RNAseq}

To simulate the conditions used for the rheology measurements, 
cell:collagen mixtures were incubated at $23^{\circ} \mathrm{C}$ for 30 and 90 min before snap freezing the reconstitution mixtures in liquid nitrogen and extracting RNA using the TRIZOL method (Thermo Scientific). Quality and integrity of RNA was assessed with the Fragment Analyzer from Advanced Analytical by using the standard sensitivity RNA Analysis Kit (DNF-471). RNA-seq libraries were prepared using a modified strand-specific, massively-parallel cDNA sequencing (RNA-Seq) protocol from Illumina, the TruSeq Stranded Total RNA (Cat.No. RS-122-2301). Libraries were sequenced on a HiSeq 4000 platform (Illumina) generating 50 bp single-end reads (30-40 Mio reads/sample). Sequence images were transformed with Illumina software BaseCaller to BCL files, which was demultiplexed to fastq files with bcl2fastq v2.17.1.14. The quality check was done using FastQC (version0.11.5, BabrahamBioinformatics). Sequence reads were aligned to the human genome reference assembly (UCSC version hg19) using Bowtie 2.0 (Langmead and Salzberg, 2012). For each gene, the number of mapped reads was counted using HTSeq-counts; and DESeq2 was used to analyze the differential expression (Anders and Huber, 2010). DEGs were identified by a $\log 2$-fold difference of 0.5 in transcript levels between the $30 \mathrm{~min}$ and $90 \mathrm{~min}$ time-points according to the Benjamini-Hochberg method for multiple comparisons (adjusted $P<0.05$ ). Gene ontology (GO) analyses were performed using DAVID (Huang da et al., 2009a; Huang da et al., 2009b). VennDiagrams were generated using BioVenn (Hulsen et al., 2008).

\subsection{Statistical analysis}

Data are displayed as mean \pm standard error of the mean (SEM). Figure captions include information about the applied statistical test methods and sample sizes. Statistical significance was assumed when $\mathrm{p}<0.05$. Statistical test were performed using GraphPad software (version 7).

\section{Acknowledgements}

This work was funded via the Collaborative Research Center SFB 937, projects A13 (F.R.) and A18 (S.L., U.P., W.H.Z.). M.T. and W.H.Z. receive additional support from SFB 1002 C04 and S01, IRTG 1816 RP12, and DZHK. We acknowledge the excellent technical assistance of Andreas Schraut, Iris Quentin, Krasimira Sharkova, Daria Reher and Monika Hoch in the generation of HES2-derived CMs, routine culture of Fibs, and Sirius red staining of slides. We are grateful to Elina Grishina for immunostaining of EHM time-series cryosections. The support by Gabriela Salinas, Orr Shomroni and the technical staff of the Transcriptome and Genome Analysis Laboratory (TAL) at the University Medical Center Göttingen is greatly acknowledged.

\section{Appendix A. Supplementary data}

Supplementary data to this article can be found online at https://doi.org/10.1016/j.pbiomolbio.2018.11.011.

\section{References}

Anders, S., Huber, W., 2010. Differential expression analysis for sequence count data. Genome Biol. 11, R106.

Beermann, J., Piccoli, M.T., Viereck, J., Thum, T., 2016. Non-coding RNAs in development and disease: background, mechanisms, and therapeutic approaches. Physiol. Rev. 96, 1297-1325.

Bonnans, C., Chou, J., Werb, Z., 2014. Remodelling the extracellular matrix in development and disease. Nat. Rev. Mol. Cell Biol. 15, 786-801.

Chuang, J.S., Zemljic-Harpf, A., Ross, R.S., Frank, L.R., McCulloch, A.D., Omens, J.H., 2010. Determination of three-dimensional ventricular strain distributions in gene-targeted mice using tagged MRI. Magn. Reson. Med. 64, 1281-1288.

Ewoldt, R.H., Hosoi, A.E., McKinley, G.H., 2008. New measures for characterizing nonlinear viscoelasticity in large amplitude oscillatory shear. J. Rheol. 52, 1427-1458.

Gao, L., Gregorich, Z.R., Zhu, W., Mattapally, S., Oduk, Y., Lou, X. Kannappan, R., Borovjagin, A.V., Walcott, G.P., Pollard, A.E., Fast, V.G., Hu, X., Lloyd, S.G., Ge, Y., Zhang, J., 2018. Large cardiac muscle patches engineered from human inducedpluripotent stem cell-derived cardiac cells improve recovery from myocardial infarction in swine. Circulation 137, 1712-1730.

Godier-Furnemont, A.F, Tiburcy, M., Wagner, E. Dewenter, M., Lammle, S., ElArmouche, A., Lehnart, S.E., Vunjak-Novakovic, G., Zimmermann, W.H., 2015. Physiologic force-frequency response in engineered heart muscle by electromechanical stimulation. Biomaterials 60, 82-91.

Godier-Furnémont, A.F.G, Tiburcy, M., Wagner, E., Dewenter, M., Lämmle, S., ElArmouche, A., Lehnart, S.E., Vunjak-Novakovic, G., Zimmermann, W.-H., 2015. Physiologic force-frequency response in engineered heart muscle by electromechanical stimulation. Biomaterials 60, 82-91.

Hanley, K.P., Oakley, F., Sugden, S., Wilson, D.I., Mann, D.A., Hanley, N.A., 2008. Ectopic SOX9 mediates extracellular matrix deposition characteristic of organ fibrosis. J. Biol. Chem. 283, 14063-14071.

Heidemann, K.M., Sageman-Furnas, A.O., Sharma, A., Rehfeldt, F., Schmidt, C.F., Wardetzky, M., 2018. Topology counts: force distributions in circular spring networks. Phys. Rev. Lett. 120, 068001.

Heidemann, K.M., Sharma, A., Rehfeldt, F., Schmidt, C.F., Wardetzky, M., 2015. Elasticity of 3D networks with rigid filaments and compliant crosslinks. Soft Matter 11, 343-354.

Hersch, N., Wolters, B., Dreissen, G., Springer, R., Kirchgeßner, N., Merkel, R., Hoffmann, B., 2013 Mar 15. The constant beat: cardiomyocytes adapt their forces by equal contraction upon environmental stiffening. Biol. Open 2 (3), 351-361. https://doi.org/10.1242/bio.20133830.

Herum, K.M., Lunde, I.G., McCulloch, A.D., Christensen, G., 2017. The soft- and hardheartedness of cardiac fibroblasts: mechanotransduction signaling pathways in fibrosis of the heart. J. Clin. Med. 6.

Huang da, W., Sherman, B.T., Lempicki, R.A., 2009a. Bioinformatics enrichment tools: paths toward the comprehensive functional analysis of large gene lists. Nucleic Acids Res. 37, 1-13.

Huang da, W., Sherman, B.T., Lempicki, R.A., 2009b. Systematic and integrative analysis of large gene lists using DAVID bioinformatics resources. Nat. Protoc. 4, $44-57$.

Huber, F., Schnauß, J., Rönicke, S., Rauch, P., Müller, K., Fütterer, C., Käs, J., 2013. Emergent complexity of the cytoskeleton: from single filaments to tissue. Adv. Phys. 62, 1-112.

Hulsen, T., de Vlieg, J., Alkema, W., 2008. BioVenn - a web application for the comparison and visualization of biological lists using area-proportional Venn diagrams. BMC Genomics 9, 488.

Irion, S., Luche, H., Gadue, P., Fehling, H.J., Kennedy, M., Keller, G., 2007. Identification and targeting of the ROSA26 locus in human embryonic stem cells. Nat. Biotechnol. 25, 1477.

Kajzar, A., Cesa, C.M., Kirchgeßner, N., Hoffmann, B., Merkel, R., 2008. Toward physiological conditions for cell analyses: forces of heart muscle cells suspended between elastic micropillars. Biophys. J. 94, 1854-1866.

Kensah, G., Roa Lara, A., Dahlmann, J., Zweigerdt, R., Schwanke, K., Hegermann, J., Skvorc, D., Gawol, A., Azizian, A., Wagner, S., Maier, L.S., Krause, A., Dräger, G., Ochs, M., Haverich, A., Gruh, I., Martin, U., 2013. Murine and human pluripotent stem cell-derived cardiac bodies form contractile myocardial tissue in vitro. Eur. Heart J. 34, 1134-1146.

Lacraz, G.P.A., Junker, J.P., Gladka, M.M., Molenaar, B., Scholman, K.T., VigilGarcia, M., Versteeg, D., de Ruiter, H., Vermunt, M.W., Creyghton, M.P., Huibers, M.M.H., de Jonge, N., van Oudenaarden, A., van Rooij, E., 2017. Tomoseq identifies SOX9 as a key regulator of cardiac fibrosis during ischemic injury. Circulation 136, 1396-1409.

Langmead, B., Salzberg, S.L., 2012. Fast gapped-read alignment with Bowtie 2. Nat. Methods 9, 357.

Li, Y., Lui, K.O., Zhou, B., 2018. Reassessing endothelial-to-mesenchymal transition in cardiovascular diseases. Nat. Rev. Cardiol.

Liaw, N.Y., Zimmermann, W.H., 2016. Mechanical stimulation in the engineering of heart muscle. Adv. Drug Deliv. Rev. 96, 156-160.

Marquez, J.P., Elson, E.L., Genin, G.M., 2010. Whole cell mechanics of contractile fibroblasts: relations between effective cellular and extracellular matrix moduli. Phil. Trans. Math. Phys. Eng. Sci. 368, 635-654.

Naito, $\mathrm{H}$, Melnychenko, I. Didié, M. Schneiderbanger, $\mathrm{K}$, Schubert, $\mathrm{P}$, Rosenkranz, S., Eschenhagen, T., Zimmermann, W.-H., 2006. Optimizing engineered heart tissue for therapeutic applications as surrogate heart muscle. Circulation 114. I-72-I-78.

Ronaldson-Bouchard, K., Ma, S.P., Yeager, K., Chen, T., Song, L., Sirabella, D., Morikawa, K., Teles, D. Yazawa, M., Vunjak-Novakovic, G., 2018. Advanced maturation of human cardiac tissue grown from pluripotent stem cells. Nature 556, 239-243.

Soong, P.L., Tiburcy, M., Zimmermann, W.H., 2012. Cardiac differentiation of human embryonic stem cells and their assembly into engineered heart muscle. Current Protocols Cell Biol. 55, 23.28.1-23.8.21

Stein, S., Luther, S., Parlitz, U., 2017. Impact of viscoelastic coupling on the synchronization of symmetric and asymmetric self-sustained oscillators. New J. Phys. 19, 063040.

Storm, C., Pastore, J.J., MacKintosh, F.C., Lubensky, T.C., Janmey, P.A., 2005. Nonlinear elasticity in biological gels. Nature 435, 191-194.

Tiburcy, M., Didie, M., Boy, O., Christalla, P., Doker, S., Naito, H., Karikkineth, B.C., El- 
Armouche, A., Grimm, M., Nose, M., Eschenhagen, T., Zieseniss, A. Katschinksi, D.M., Hamdani, N., Linke, W.A., Yin, X., Mayr, M. Zimmermann, W.H., 2011. Terminal differentiation, advanced organotypic maturation, and modeling of hypertrophic growth in engineered heart tissue. Circ. Res. 109, 1105-1114.

Tiburcy, M., Hudson, J.E., Balfanz, P., Schlick, S., Meyer, T., Chang Liao, M.-L. Levent, E., Raad, F., Zeidler, S., Wingender, E., Riegler, J., Wang, M., Gold, J.D., Kehat, I., Wettwer, E., Ravens, U., Dierickx, P., van Laake, L.W., Goumans, M.J. Khadjeh, S., Toischer, K., Hasenfuss, G., Couture, L.A., Unger, A., Linke, W.A. Araki, T., Neel, B., Keller, G., Gepstein, L., Wu, J.C., Zimmermann, W.-H., 2017 Defined engineered human myocardium with advanced maturation for applications in heart failure modeling and repair. Circulation 135, 1832-1847.

Tiburcy, M., Meyer, T., Soong, P.L., Zimmermann, W.H., 2014. Collagen-based engineered heart muscle. Methods Mol. Biol. 1181, 167-176.

Vantler, M., Karikkineth, B.C., Naito, H., Tiburcy, M., Didié, M., Nose, M. Rosenkranz, S., Zimmermann, W.-H., 2010. PDGF-BB protects cardiomyocytes from apoptosis and improves contractile function of engineered heart tissue. J. Mol. Cell. Cardiol. 48, 1316-1323.
Viereck, J., Bang, C., Foinquinos, A., Thum, T., 2014. Regulatory RNAs and paracrine networks in the heart. Cardiovasc. Res. 102, 290-301.

Villasante, A., Marturano-Kruik, A., Ambati, S.R., Liu, Z., Godier-Furnemont, A Parsa, H., Lee, B.W., Moore, M.A., Vunjak-Novakovic, G., 2016. Recapitulating the size and cargo of tumor exosomes in a tissue-engineered model. Theranostics 6 , 1119-1130.

Wales, D.J., Doye, J.P.K., 1997. Global optimization by basin-hopping and the lowest energy structures of Lennard-Jones clusters containing up to 110 atoms. J. Phys. Chem. 101, 5111-5116.

Weinberger, F., Breckwoldt, K., Pecha, S., Kelly, A., Geertz, B., Starbatty, J., Yorgan, T., Cheng, K.H., Lessmann, K., Stolen, T., Scherrer-Crosbie, M., Smith, G. Reichenspurner, H., Hansen, A., Eschenhagen, T., 2016. Cardiac repair in Guinea pigs with human engineered heart tissue from induced pluripotent stem cells. Sci. Transl. Med. 8, 363ra148.

Zhang, D., Shadrin, I.Y., Lam, J., Xian, H.-Q., Snodgrass, H.R., Bursac, N., 2013. Tissueengineered cardiac patch for advanced functional maturation of human ESCderived cardiomyocytes. Biomaterials 34, 5813-5820. 


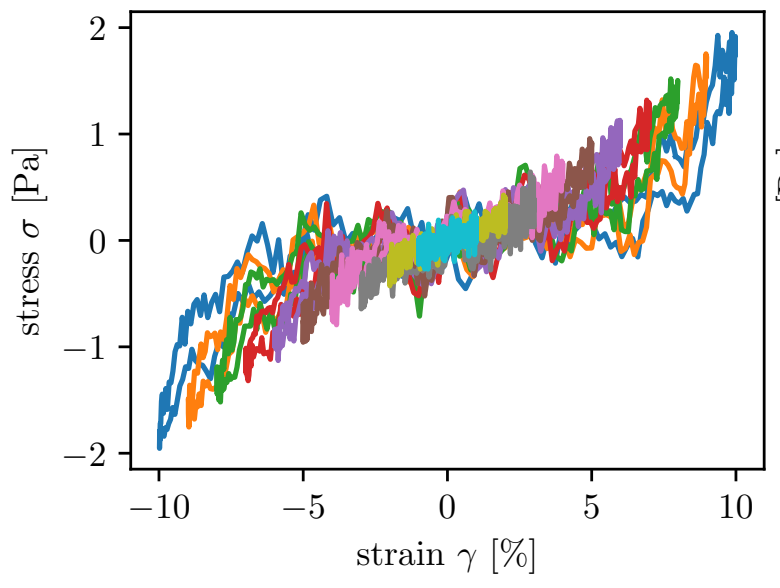

(a) $\mathrm{CP} 25-2$

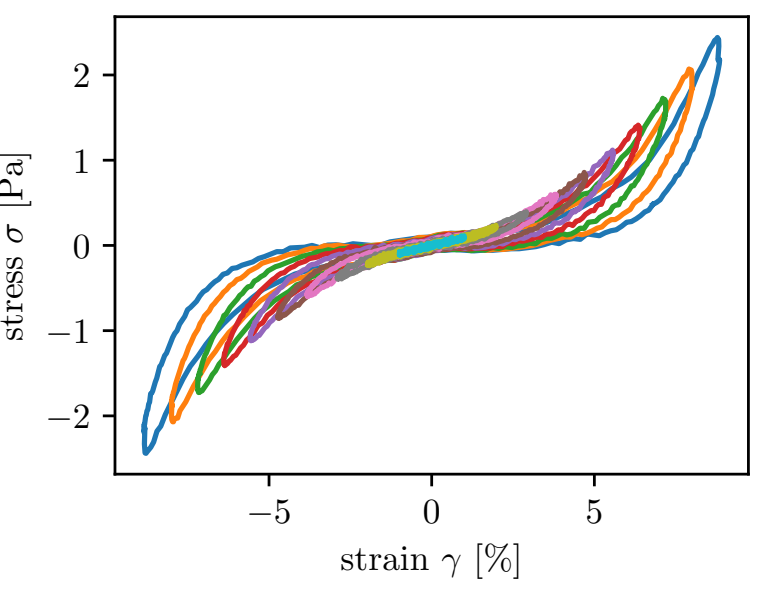

(b) CP50-1

Figure 4.1 Lissajous figures of collagen hydrogels without cells. (a) Stress-strain curves of sine LAOS measurements with $1 \% \leq \gamma_{0} \leq 10 \%$ using the conical measuring system CP25-2 as in the experiments in the previous section. Different colors indicate different strain amplitudes. (b) Shows the same measurements on a hydrogel using the same collagen (17CSA03) but using the larger measuring system CP50-1. The doubled radius results in higher total momenta, thus reducing noise significantly.

\subsection{Nonlinear stress response of collagen}

After having found an ad-hoc model for the nonlinear stress response of collagen hydrogels with and without cells in the previous section, this model has to be tested with further experimental data including the stress responses to nonsinusoidal strain input. In the end, the model should replace the linear Maxwell element representing the ECM in the mathematical model for CM coupled by the ECM used in chapter 3 by a more realistic description. The ad-hoc model thus has to predict the stress response to the strain exerted by the contracting CM (cf. figure $2.2 \mathrm{~b}$ ). So far, the model has only been tested with sine waveforms at a frequency of $1 \mathrm{~Hz}$. This will be extended to sine waveforms at larger frequencies and nonsinusoidal strain input in the following.

We found in [1] that the qualitative strain-stiffening behavior is present in all types of hydrogels (no cells, with HFF, with CM, or with both types (EHM)), although it is most pronounced in hydrogels without cells. To be independent of cell performance and to be able to conduct a larger number of experiments more easily, I decided not to include cells into the measurements used to test the model. The presence of cells de facto limited the possible sample volumes and thus the choice of the rheometer probes (cf. table 2.1) - we tried to use as few cells as possible for the hydrogels, since after having been used in the rheometry, hydrogels and the cells were discarded and were lost for further measurements. Another side effect was that the protocol of hydrogel preparation could be changed from the EHM protocol to the more reliable PBS protocol introduced in section 2.4.2. This is specifically important for comparing different batches of collagen in section 4.3. 


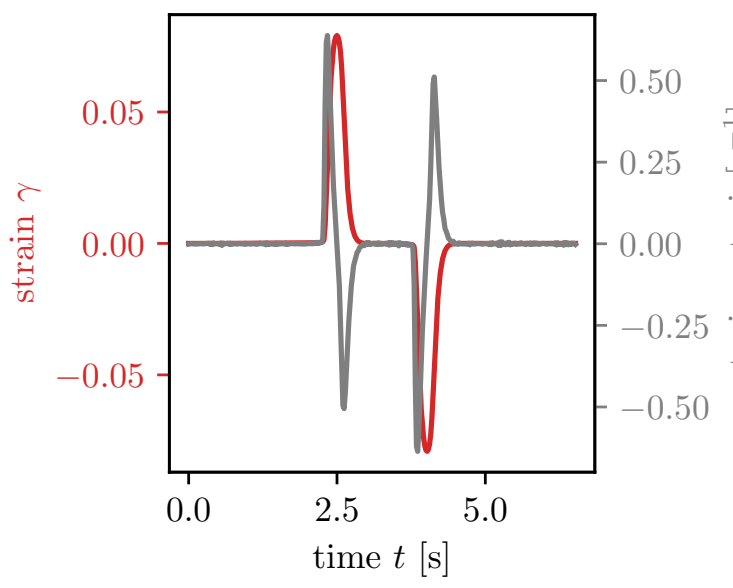

(a) $\gamma(t)$ and $\dot{\gamma}(t)$

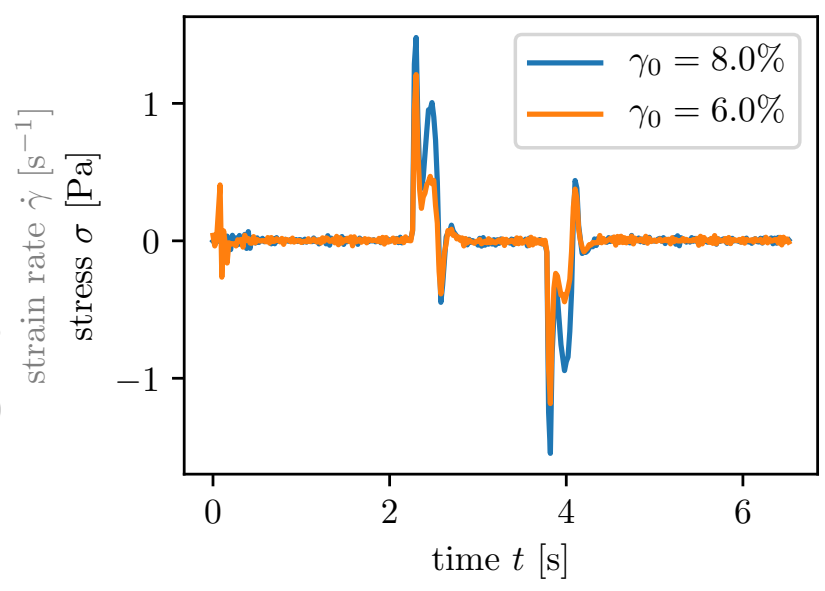

(b) stress response

Figure 4.2 Strain input from contraction curves. (a) shows strain $\gamma$ and strain rate $\dot{\gamma}$ as measured by the rheometer for $\gamma_{0}=8 \%$ with the input data from figure 2.10. (b) Stress responses to this contraction strain input for two exemplary strain amplitudes $6 \%$ and $8 \%$.

Larger sample volumes allowed me to use the larger measuring system CP50-1 with $2.5 \mathrm{~cm}$ radius instead of the $\mathrm{CP} 25-2$ with a radius of $1.25 \mathrm{~cm}$. The benefit of the larger radius becomes apparent in figure 4.1. Figure 4.1a shows Lissajous curves for strain amplitudes $1 \% \leq \gamma_{0} \leq 10 \%$ similar to figures 6 and 7 in section 4.1 recorded with the smaller probe. Since the hydrogel used here is softer than the one used in the previous section (cf. also section 4.3), the absolute stress values are not as high as in the previous cases without cells. Thus noise becomes relevant and would have to be filtered as was done previously using Fourier filtering. Figure 4.1b shows a similar measurement on a hydrogel using the same collagen (17CSA03) but with the larger probe CP50-1. Due to the higher absolute momenta acting on a probe with a larger radius, noise is almost absent, allowing the direct use of the measured data without further processing. Thus, in all following experiments, the PBS protocol and the CP50-1 measuring system with larger sample volume are used.

\subsubsection{Sinusoidal and nonsinusoidal strain input}

With the adaptions to the protocol introduced above I started measuring the stress response of the collagen hydrogels to nonsinusoidal strain inputs, namely the triangle, square, and sawtooth waveforms and the strain input obtained from the contraction curve as explained in section 2.4.3. Exemplary results of the latter are shown in figure 4.2. The data shown in figure 2.10 with a sampling rate of $30 \mathrm{~Hz}$ is used as strain input. The strain $\gamma(t)$ and strain rate $\dot{\gamma}(t)$ that were measured subsequently by the rheometer are shown in figure $4.2 \mathrm{a}$ and match the input data. In contrast to the predefined nonsinusoidal inputs (cf. figure 2.9), desired and actual strain amplitude match $\left(\gamma_{0}=8 \%\right.$ in the case shown here). The subsequent stress response is displayed in figure $4.2 \mathrm{~b}$ for two exemplary strain amplitudes $\gamma_{0}=6 \%$ and $\gamma_{0}=8 \%$. In both cases, 


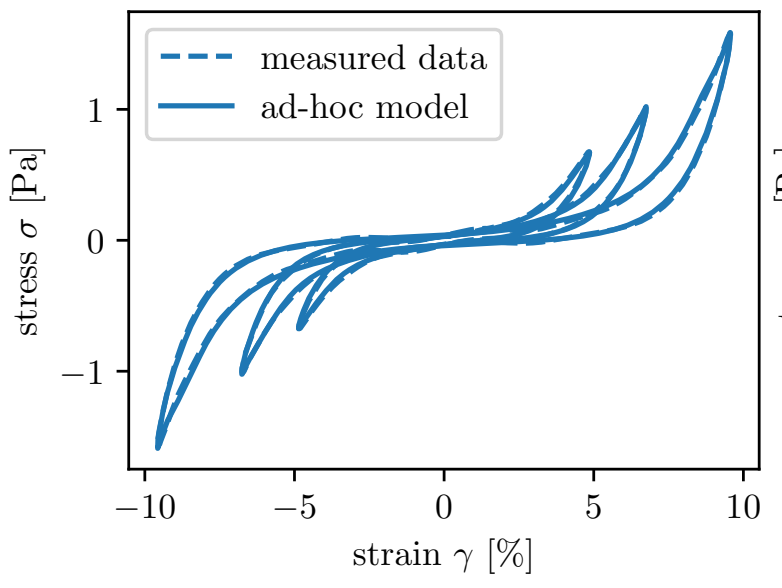

(a) Sinusoidal strain

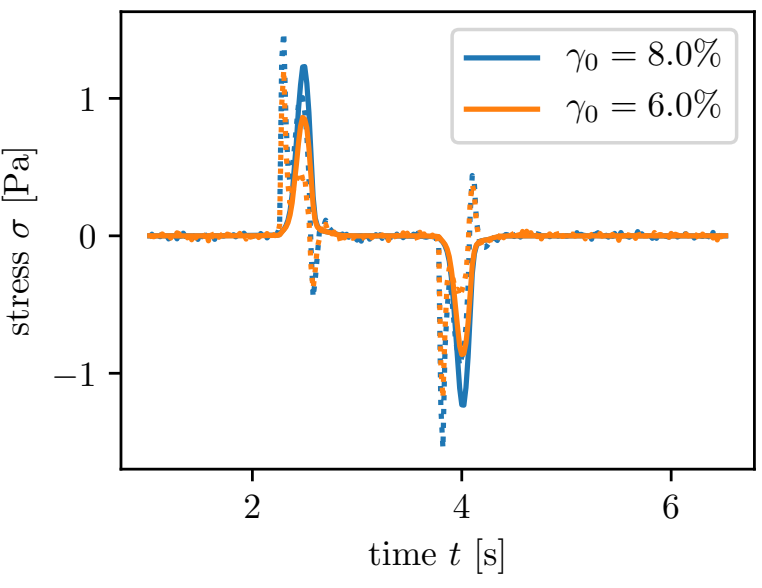

(b) Contraction strain

Figure 4.3 Measured and predicted stress response. (a) As in [1], the ad-hoc model reproduces the measured stress response (dashed lines) for all strain amplitudes in the case of sinusoidal strain. (b) With the model coefficients obtained by fitting to the data from the sinusoidal strain input, the stress response to the contraction strain input is not correctly predicted (measured data indicated by dotted lines, same data as in figure $4.2 \mathrm{~b}$ ). Model coefficients are included in appendix C.2 and table C.2 therein.

the stress response to the contraction excursion with $\gamma>0$ first shows an initial peak that quickly decreases followed by a second maximum and a minimum that reaches negative stress values for positive strain. The initial peak and final minimum seem to follow closely the dynamics of the strain rate while the second maximum seems to be linked closer to the strain itself and shows a stronger dependence on the strain amplitude. I observed a similar behavior when using square and sawtooth waveforms at their respective steep ascends and descends. The response to the excursion with $\gamma<0$ is symmetrical to the one to $\gamma>0$. In the beginning of the time series of $\sigma(t)$ for $\gamma_{0}=6 \%$, there is a short time interval in which I observed nonzero stress despite $\gamma=0$ and $\dot{\gamma}=0$. This is likely to be either a remainder of a previous measurement or of an internal equilibration phase of the instrument itself in between the measurements that did not entirely finish. Clearly it is not a response to the actual strain input. To exclude any influence of this I discard the first second of all these time series in the following.

\subsubsection{Ad-hoc model and stress response to higher frequencies}

The measured stress response to the strain input obtained from the contraction curve can now be used to test the predictions of the ad-hoc model (or any other model, of course). To this end, I determine the model coefficients for each sample separately from the sine LAOS measurements. As in section 4.1, this is done by finding the minimum of the difference between modelled and measured stress response using the basin-hopping algorithm. The coefficients obtained that way are shown in table C.2. 


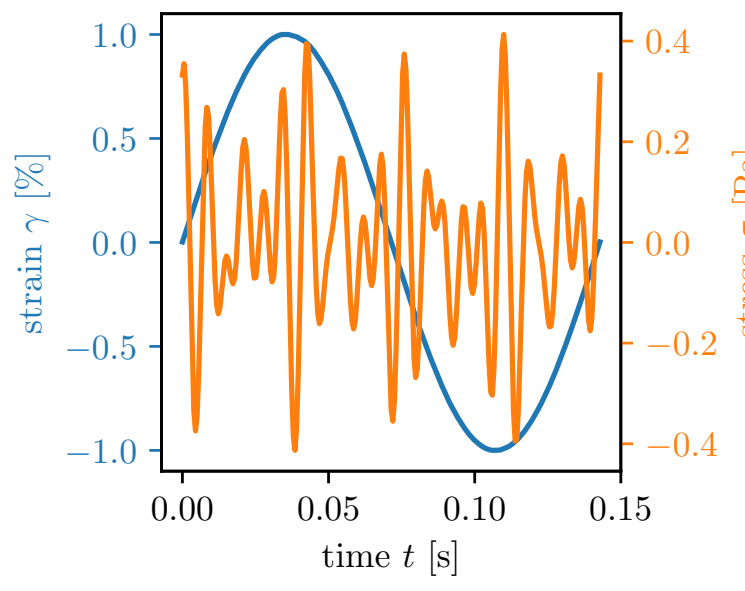

(a) $\gamma_{0}=1 \%$

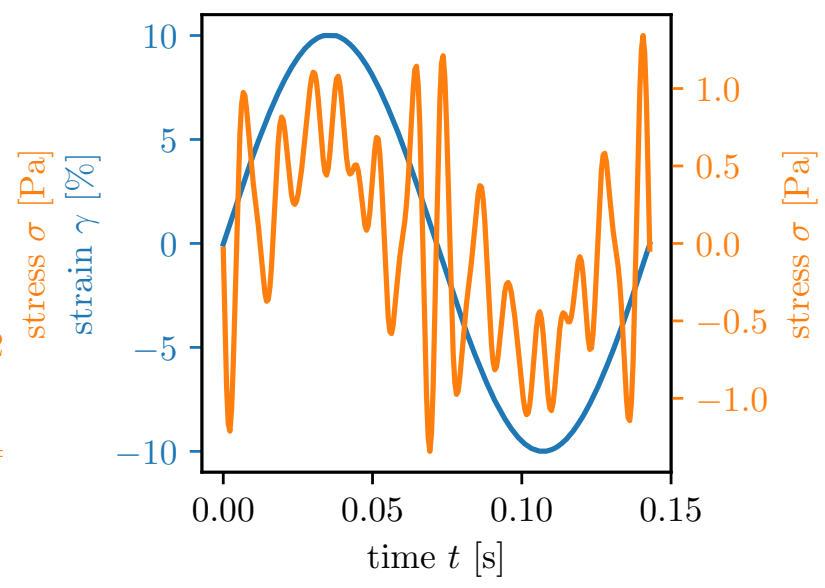

(b) $\gamma_{0}=10 \%$

Figure 4.4 Sine LAOS at a frequency $f=7 \mathrm{~Hz}$ for amplitudes $\gamma_{0}=1 \%$ (a) and $\gamma_{0}=10 \%$ (b). In both cases, the measured stress response is lost in high-frequency fluctuations.

See appendix C.2 and equation C.2 for more information about the ad-hoc model. The resulting modelled stress for the sinusoidal strain is shown as solid line in figure 4.3a for three different strain amplitudes. As before, the ad-hoc model is able to reproduce the measured stress response (indicated by dashed lines) in the sine LAOS case. This is not the case for the contraction curve input as is shown in figure $4.3 \mathrm{~b}$. Here, for the same sample as in figure $4.3 \mathrm{a}$, the model is solved with strain and strain rate stemming from the contraction curve (cf. figure 4.2a) and the coefficients obtained from the sine LAOS measurements. The resulting predicted stress is shown in figure $4.3 \mathrm{~b}$ for two exemplary strain amplitudes $\gamma_{0}=6 \%$ and $\gamma_{0}=8 \%$ as solid, the measured stress response as dotted lines. Clearly, modeled and measured stress do not coincide; the prediction from the model does not show the quick excursions of the measured stress and only one maximum per deformation instead.

In general, there could be arbitrarily many reasons for the model prediction to deviate from the measured data. The training data from the sine LAOS measurements might be ill-suited for the contraction strain input or the basin-hopping algorithm found a local, but not the global minimum, although longer optimization showed no improvement. Last but not least, the model, i.e., the ad-hoc third order polynomial could be insufficient to correctly model the stress response altogether and other models should be used.

Regardless of the details of the model, there is reason to believe that the sine LAOS measurements with frequencies of $1 \mathrm{~Hz}$ are not sufficient to also model the stress response to the contraction input. The flanks of the contraction curves are steeper than those of a sine at $1 \mathrm{~Hz}$ (cf. figure $2.2 \mathrm{~b}$ ); in fact, when attempting to do a Fourier decomposition one needs at least modes up to $8 \mathrm{~Hz}$ to correctly describe the contraction curve. That is why I extended the LAOS measurements to include strain input up to $10 \mathrm{~Hz}$.

Exemplary results of a sine LAOS measurement of a hydrogel with collagen 17CSA03 
at $f=7 \mathrm{~Hz}$ are shown in figure 4.4 for strain amplitudes $\gamma_{0}=1 \%$ and $\gamma_{0}=10 \%$, i.e., in the linear and in the strain-stiffening regime, respectively. In both cases, the measured stress response is lost under high-frequency noise. I observed the same type of noise in all LAOS measurements with frequencies above $2 \mathrm{~Hz}$. Whether it is due to the lack of control features that had to be switched off for the recording of $\gamma(t)$ and $\sigma(t)$ or due to other issues with the instrument's hardware or software remains unclear. Communication with the manufacturer Anton Paar lead to no improvement but it became clear that this type of measurement - possibly nonlinear with direct strain and stress recording on very soft samples (compared to usual usage of these rheometers with linear moduli on the order of at least $\mathrm{kPa}$ ) is on the very limits of or possibly beyond the resolution of the instrument. According to the manufacturer, it is unlikely that the stress signal in this type of measurement will be more clear using any of their shear rheometers.

Since the measured stress-response of a pure sine waveform with frequencies higher than $1 \mathrm{~Hz}$ is overlaid with the measurement artifacts visible in figure 4.4, it is unclear whether I can trust any measured stress to a strain input including comparably high modes or comparably large strain rates. Specifically, it is unclear whether the fast excursions following the extrema in strain rate visible in the stress-response to the contraction input (cf. figure 4.2b) but also present in sawtooth and square measurements are actually part of the stress-response or rather measurement artifacts. With this uncertainty, the measured data are unfit to test the predictions of my ad-hoc model or any other model. The question whether the ad-hoc model is suitable to replace the Maxwell elements in the mathematical model of coupled cardiomyocytes by a more realistic description of the ECM thus cannot be answered here.

\subsubsection{Prestress measurements}

As described in the previous section, the attempt of directly measuring the stress response to a strain input taken from the contraction curves from my simulations remained inconclusive and no description of the stress response of the ECM could be obtained. Other possibilities of measuring the nonlinear stress-strain relation are prestrain and prestress measurements as explained in section 2.4.3. Since these experiments are only possible when conducting stress-controlled measurements with my experimental setup, I conducted prestress measurements. I used four different values of the prestress, $\sigma_{\text {pre }} \in[0.1 \mathrm{~Pa}, 0.5 \mathrm{~Pa}, 0.9 \mathrm{~Pa}, 1.3 \mathrm{~Pa}]$ to probe the nonlinearity and a stress amplitude of $\sigma_{0}=0.05 \mathrm{~Pa}$ for the oscillations performed on top of the prestress. The oscillations were set to frequencies between $1 \mathrm{~Hz}$ and $4 \mathrm{~Hz}$ to cover a part of the frequency range that proved problematic in the LAOS measurements described above. In the following I describe the results of my preliminary prestress measurements.

Since it is not possible for our rheometer to simultaneously record the strain due to the oscillation and the one resulting from the application of $\sigma_{\text {pre }}$, it is necessary to first calibrate the prestrain $\gamma_{\text {pre }}$. As explained in section 2.4.3 this is done by applying a constant stress $\sigma_{\text {pre }}$ without further oscillation and measuring the corresponding strain. The result of such a calibration is shown in figure $4.5 \mathrm{a}$ in black. Each $\gamma_{\text {pre }}$ is obtained 


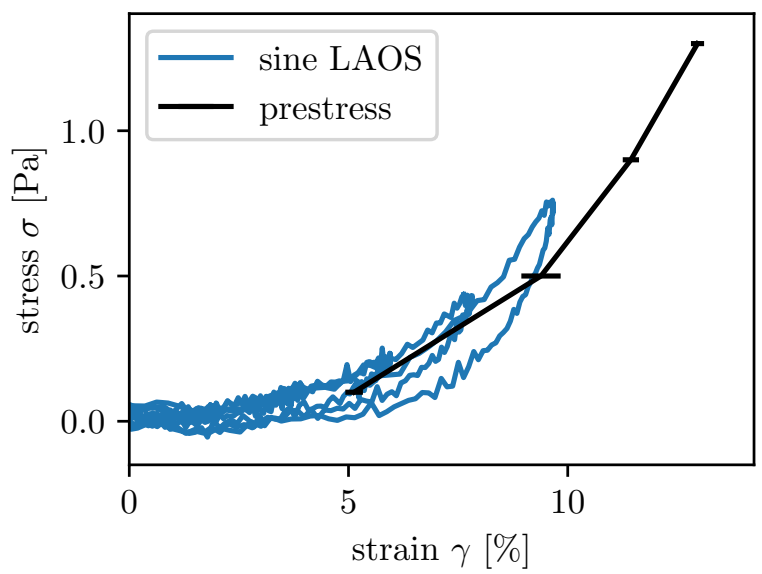

(a) Calibration

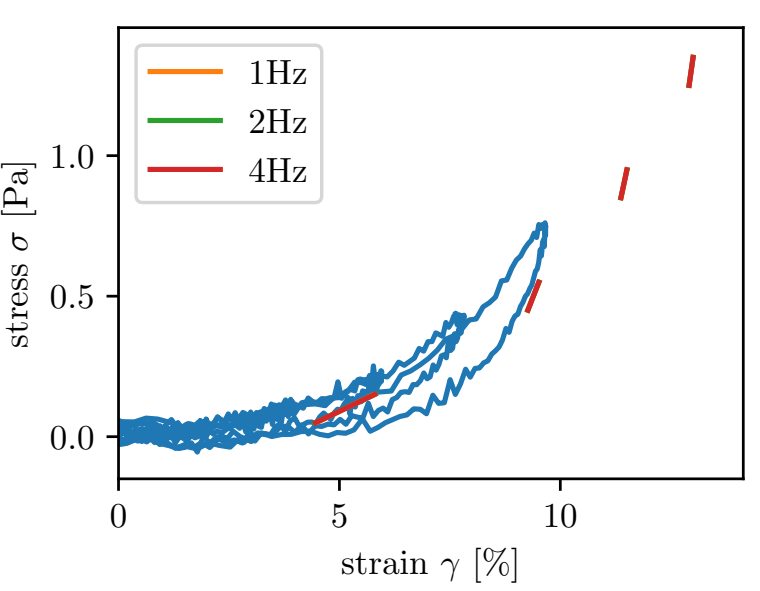

(b) Prestress

Figure 4.5 Prestress measurements with the $\gamma>0$ branch of the sine LAOS measurements on the same sample. $\gamma_{\text {pre }}$ has to be calibrated from measuring the strain resulting from a constant stress $\sigma_{\text {pre }}(\mathrm{a})$. The resulting $\sigma_{\text {pre }}\left(\gamma_{\text {pre }}\right)$ is shown in black. Each value of $\gamma_{\text {pre }}$ is obtained by averaging over five single measurements, error bars indicate the standard deviation. In the range where LAOS measurement and prestress overlap, they coincide relatively well. (b) shows the response to the stress oscillations on top of the prestress for three different frequencies $1 \mathrm{~Hz}, 2 \mathrm{~Hz}$, and $4 \mathrm{~Hz}$. The response is approximately independent of the frequency of oscillation and the lines coincide. The stress-strain curves from the prestress measurements roughly correspond to the slope of the Lissajous curve.

by averaging of 5 single measurements. The error bars indicate the corresponding standard deviation. In blue I show the Lissajous figures as in the previous sections stemming from the sine LAOS measurement at $1 \mathrm{~Hz}$ for the same sample (collagen 18CB024001) for comparison. The latter show the stress response to oscillatory strain, i.e., to a dynamical strain input. In contrast, stress and strain are constant in the calibration measurement. Still, in the stress and strain range in which there is data from both measurements, i.e., $5 \% \leq \gamma \leq 10 \%$ and $0.1 \mathrm{~Pa} \leq \sigma \leq 0.5 \mathrm{~Pa}$ they coincide quite well.

For the four values of $\sigma_{\text {pre }}$ and the three frequencies, the oscillatory stress is added. In order to have all control features of the instrument in place, this is done without recording the time series of $\gamma(t), \dot{\gamma}(t)$, and $\sigma(t)$. Instead, the (linear) response to the oscillation is again characterized by the averaged quantities of storage and loss modulus $G^{\prime}$ and $G^{\prime \prime}$. This is why $\sigma_{0}$ has to be chosen small enough for the response to the oscillations to be linear. $G^{\prime}$ and $G^{\prime \prime}$ may depend nonlinearly on $\sigma_{\text {pre }}$ thus reflecting the strain-stiffening behavior. Figure $4.5 \mathrm{~b}$ shows the results of the oscillatory prestress measurements. At each point $\left(\gamma_{\text {pre }}, \sigma_{\text {pre }}\right)$ found by the aforementioned calibration, the strain response to the oscillatory stress is shown as obtained from the local moduli and the maximum strain amplitude $\Delta \gamma_{0}$ due to the oscillation. Since at the maximum strain in the sinusoidal oscillation, the strain rate vanishes, the lines indicating the 


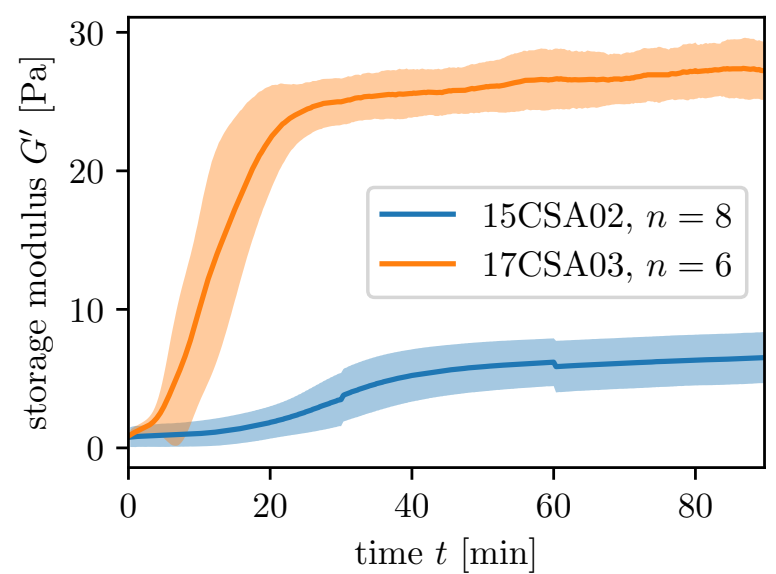

Figure 4.6 Average storage modulus $G^{\prime}$ of collagen batches 15CSA02 and 17CSA03 used for the experiments in [1] with the EHM protocol without cells. The gelation 17CSA03 sets in earlier, it reaches plateau faster and at much larger values. The data for 17CSA03 is the same as in figure 4 of section 4.1. Shaded regions indicate the standard deviation. The small "steps" in the curve for 15CSA02 stem from measurements that lasted only 30 min and 60 min, respectively.

local stress-strain relation at $\left(\gamma_{\text {pre }}, \sigma_{\text {pre }}\right)$ are found as

$$
\sigma\left(\Delta \gamma ; \gamma_{\text {pre }}\right)=\sigma_{\text {pre }}+G^{\prime}\left(\sigma_{\text {pre }}\right) \Delta \gamma ; \quad \text { where }-\Delta \gamma_{0} \leq \Delta \gamma \leq \Delta \gamma_{0}
$$

Note that this is not the actual stress-strain relation since this would depend on the strain rate, too, and would resemble an ellipsoid rather than a straight line (cf. figure 2.7a). However, the end points of the lines defined above coincide with the end points of the ellipsoid. The resulting lines roughly correspond to the slopes of the Lissajous figures (or rather, their elastic part, cf. figure 2.7b) in the range in which both data sets overlap. Interestingly, at least in the small frequency range from $1 \mathrm{~Hz}$ to $4 \mathrm{~Hz}$, the pre-stress measurements are independent of the frequency.

\subsection{Linear rheology as a means of collagen compar- ison}

When conducting the experiments on collagen with and without cells that were used in [1] for 17CSA03, we did the same measurements with an older collagen batch, 15CSA02, at first. In January and February 2018 we started experiencing difficulties in initiating gelation in 15CSA02. If it occurred at all, it often was too weak for the cell-dependent remodelling by fibroblasts seen before and in the - at that time new collagen batch 17 CSA03. Since 15 CSA02 was almost two and a half years old at the beginning of 2018 (cf. appendix B and table B.1 therein), we concluded it had deteriorated too far and used 17CSA03 for the subsequent experiments the results of which were published in [1]. Not only did the new collagen 17CSA03 polymerize more 


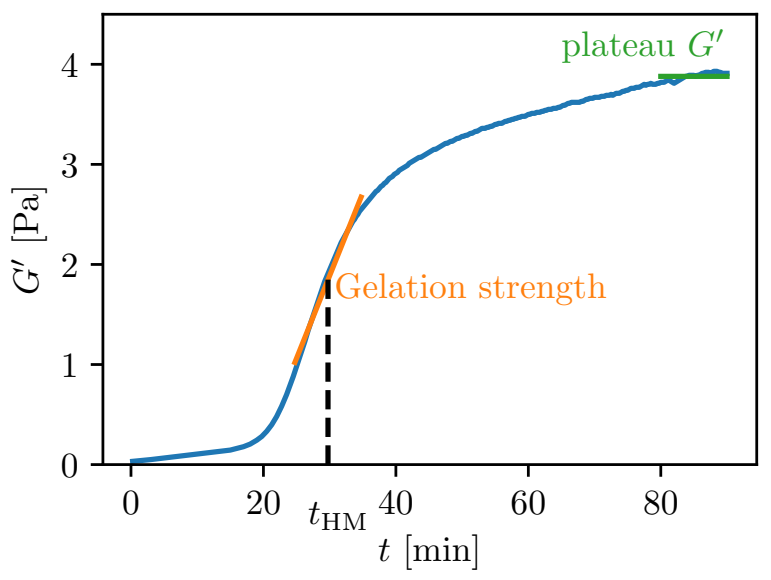

Figure 4.7 Characteristic quantities of the gelation process sketched at an exemplary time sweep of the storage modulus $G^{\prime}$ of collagen batch 18CB024003 with PBS protocol. The plateau value of $G^{\prime}$ indicated in green is obtained by taking the mean value of $G^{\prime}$ over the final 10 min of the time sweep. The point in time when $G^{\prime}$ crosses half its plateau value is denoted by $t_{\mathrm{HM}}$. I define the gelation strength as the slope of the tangent to $G^{\prime}(t)$ at $t=t_{\mathrm{HM}}$ which is found by a linear fit to $G^{\prime}(t)$ for $t_{\mathrm{HM}}-5 \mathrm{~min} \leq t \leq t_{\mathrm{HM}}+5 \mathrm{~min}$.

reliably, it also did so faster and to higher values of storage and loss modulus than 15CSA02 even when still polymerizing without problems in 2017. This is shown in the case of the storage modulus $G^{\prime}$ in figure 4.6 that shows the averages over all time sweeps ( $n=8$ measurements for 15CSA02, $n=6$ for 17CSA03) of the two collagen batches. As the loss modulus displays the same behavior (cf. figures 3 and 4 in section 4.1 and [1]), I will concentrate solely on $G^{\prime}$ in the following.

Because S. Schlick and M. Tiburcy, UMG, observed differences in EHM performance in the EHM produced using the two batches, we hypothesized that there might be a link between the gelation properties of the collagen used for the EHM and the performance of the EHM itself - measured, e.g., in force of contraction or arm thickness in case of EHM rings (cf. [15]). In order to test this hypothesis, I conducted further rheological experiments on different collagen batches in fall 2018 and again in summer 2019 (see also appendix B). All batches that were compared are listed in table 2.3. Since on the one hand, no cells were present in these measurements and, on the other hand, as much control as possible over the $\mathrm{pH}$ of the collagen hydrogels was desirable for the comparison to be meaningful, I switched the protocol for preparing the collagen hydrogels to the PBS protocol described in section 2.4.2. In order to quantify the gelation strength of the collagen hydrogels, I used the quantities sketched in figure 4.7. The plateau value of the storage modulus $G^{\prime}$ as a measure for the final stiffness of the gel is determined by averaging over $G^{\prime}$ over the final $10 \mathrm{~min}$ of the time sweep. From this, the time of half-plateau $t_{\mathrm{HM}}$ is determined as the point in time when $G^{\prime}(t)$ crosses half its plateau value. This crossing always falls into the gelation phase (cf. figure 4D in [1]), so the slope of $G^{\prime}(t)$ at $t=t_{\mathrm{HM}}$ is a good indicator for the strength of gelation. It is determined by a linear fit to $G^{\prime}(t)$ for $t_{\mathrm{HM}}-t_{\mathrm{fit}} \leq t \leq t_{\mathrm{HM}}+t_{\mathrm{fit}} \cdot t_{\mathrm{fit}}=5 \mathrm{~min}$ proved 


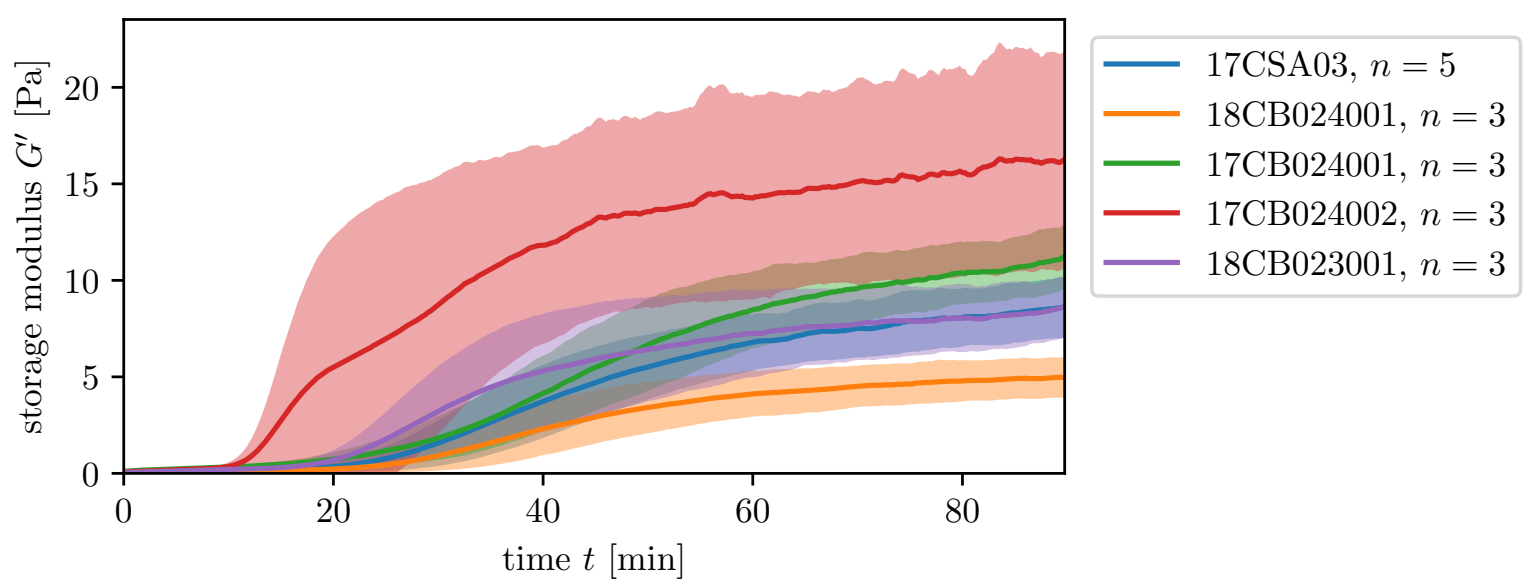

Figure 4.8 Averaged time sweeps of the storage modulus $G^{\prime}$ of the different collagen batches. Shaded regions indicate standard deviation. All hydrogels were prepared with the PBS protocol. Most of the hydrogels show comparable values of $G^{\prime}$ on average except for 18CB024001 which polymerized weaker and slower on average. Values for 17CB024002 varied strongly with two measurements being close to the other batches and one significantly stiffer.

suitable here. Since I observed a decrease in polymerization strength in the hydrogels between fall 2018 and summer 2019, which is discussed in appendix B, I concentrate on data obtained in 2018 in the following. Qualitatively, the observed behavior was the same in both series of experiments, but at lower values of $G^{\prime}$ in 2019 compared to 2018.

Figure 4.8 shows the averaged time sweeps of $G^{\prime}$ for collagen hydrogels produced with all respective collagen batches with standard deviations indicated by the shaded regions. While, on average, most of the batches showed similar results in $G^{\prime}, 18 \mathrm{CB} 024001$ consistently reached lower values. Hydrogels produced with 17CB024002 showed a relatively large variation whereby two hydrogels reached values close to the other batches and one proved significantly stiffer. Note that 17CSA03 shows a decrease in $G^{\prime}$ compared to section 4.1 and figure 4.6. This may partially be attributed to the change in protocol, but also to some extent to the ageing of the collagen (see appendix B). All in all, the values of $G^{\prime}$ are comparable to those of $15 \mathrm{CSA} 02$ with the EHM protocol in 2017 (cf. figure 4.6).

The same trends are visible in figure 4.9 showing the weighted averages of the plateau modulus for the five batches. As the weight for each plateau $G^{\prime}$, i.e., the mean of $G^{\prime}$ over the final 10 minutes of the time sweep, I use the standard deviation of $G^{\prime}$ in the same period of time to account for the fluctuations in each individual time sweep. Thus the values deviate slightly from the pure averages in figure 4.8, especially for the highly fluctuating 17CB024002. Error bars indicate the propagated errors from the weighted averages. 18CB024001 clearly shows the weakest plateau below $5 \mathrm{~Pa}$ while the other batches reach plateaus between $8 \mathrm{~Pa}$ and $10 \mathrm{~Pa}$, or in the case of $17 \mathrm{CB} 024002$ around $12 \mathrm{~Pa}$.

Similar comparisons are shown in figure 4.10a with the gelation strength and figure $4.10 \mathrm{~b}$ displaying the half-plateau times. In both cases 17CB024002 stands 


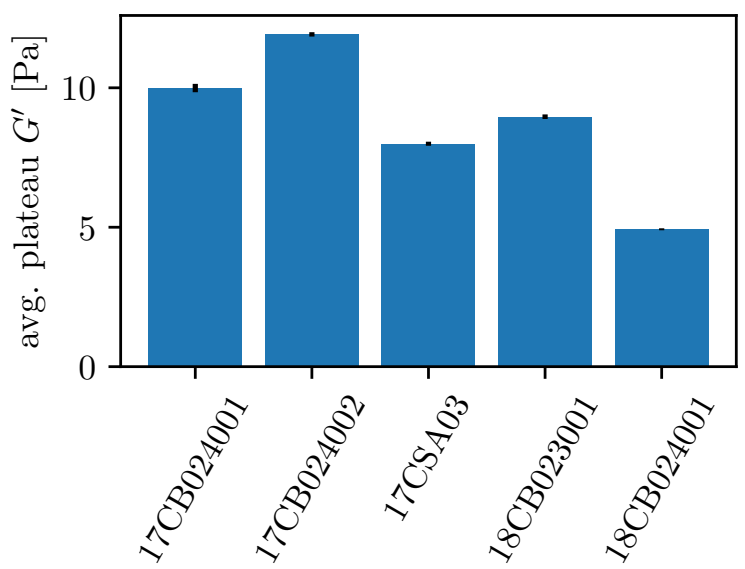

Figure 4.9 Weighted averages of plateau $G^{\prime}$ for the hydrogels shown in figure 4.8. As above, most gels remain at plateau values between $8 \mathrm{~Pa}$ and $10 \mathrm{~Pa}$; $17 \mathrm{CB} 024002$ remaining above $10 \mathrm{~Pa}$ and 18CB024001 below $5 \mathrm{~Pa}$.

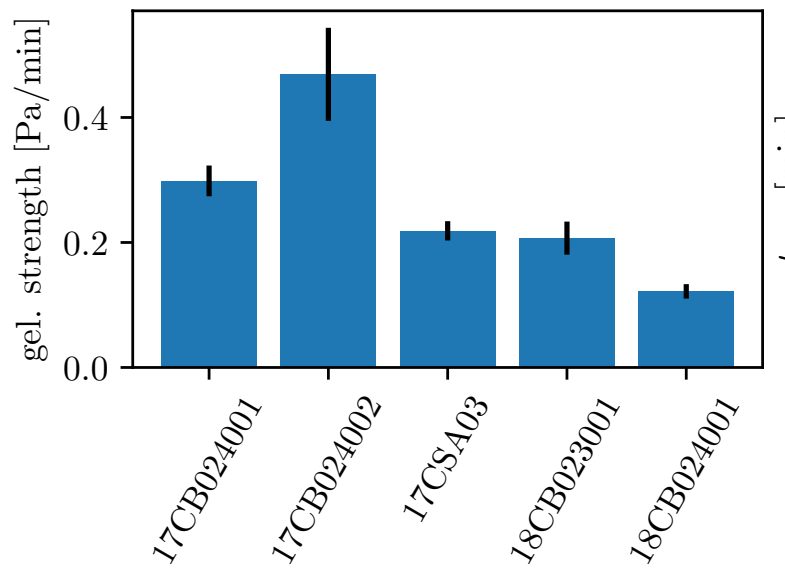

(a) Gelation strength

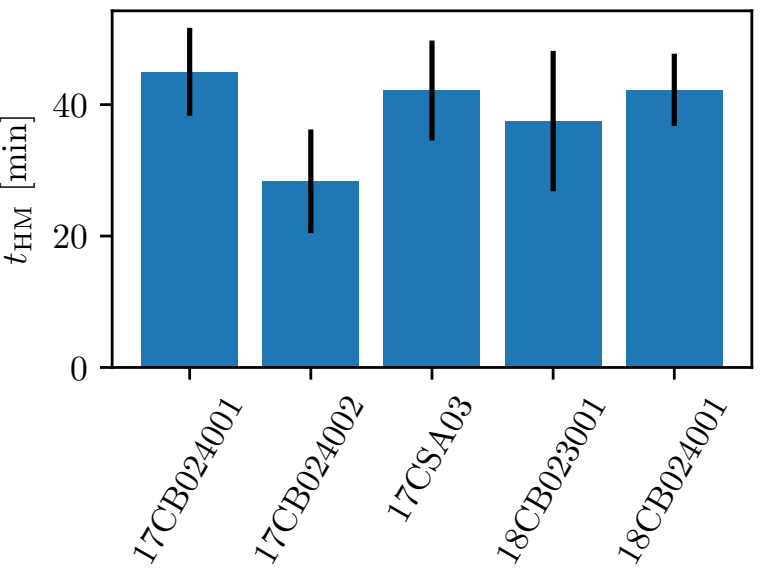

(b) Half-plateau times

Figure 4.10 Gelation strength (a) and half-plateau times $t_{\mathrm{HM}}(\mathrm{b})$ for the five collagen batches showing an increased gelation strength at earlier times in case of 17CB024002. The gelation strength shows roughly a similar trend as the plateau modulus. Interestingly, the less stiff batch 18CB024001 reaches the half value of its (lower) plateau on average at around the same time as the other gels. 
out with the strongest gelation of $(0.47 \pm 0.08) \mathrm{Pa} / \mathrm{min}$ while the other four remain below $0.3 \mathrm{~Pa} / \mathrm{min}$, and with the shortest time $t_{\mathrm{HM}}=(28 \pm 8) \mathrm{min}$ versus over $40 \mathrm{~min}$ in the other four batches. Interestingly, while showing the weakest gelation strength of $(0.12 \pm 0.02) \mathrm{Pa} / \mathrm{min}, 18 \mathrm{CB} 024001$ does not differ from the remaining three batches in terms of $t_{\mathrm{HM}}$.

Seeing the differences in polymerization between the hydrogels prepared with the different batches of collagen, one should now observe differences in EHM performance if our initial hypothesis was true. The relatively soft 18CB024001 for example might result in a reduced force of contraction or less reliable EHM production in general. No such differences were observable in EHM growth (M. Tiburcy, personal communication), however, and no clear correlation between the polymerization properties of the gels and the performance of the engineered tissue could be established. 


\section{Chapter 5}

\section{Discussion and outlook}

This thesis presented two aspects of studying oscillating cardiomyocytes coupled mechanically via the extracellular matrix. Their synchronization behavior was investigated numerically in a qualitative model of viscoelastically coupled excitable oscillators while the mechanical properties of the ECM were studied in rheological experiments. Results of both aspects have been part of the publications [33] and [1], respectively, and were already discussed in the corresponding sections 3.1 and 4.1. In the following they will be discussed again in the context of the results not part of the respective publications. I will then give an outlook on possible future work concerning the combination of the results of the numerical simulations and matrix rheology.

\subsection{Simulating viscoelastically coupled excitable os- cillators}

As discussed in section 3.1, I introduced a novel system of viscoelastically coupled excitable oscillators as a means of modelling mechanically coupled CM, motivated by the setting of tissue engineering and EHM. There, in-phase synchronization at frequencies around $1 \mathrm{~Hz}$ is observed in healthy engineered tissue [15] and indeed, even deemed necessary for the successful growth of the tissue ${ }^{1}$. The simulations agree with these observations in the sense, that they, too, show the tendency to synchronization for stiffer, i.e., more elastic and less fluid ECM. Qualitatively, this is the parameter regime to which the ECM is observed to evolve in our rheological measurements. The fact that the presence of fibroblasts enhances this development and is found to be necessary in EHM development [57] further supports the idea of mechanical synchronization being possible in early EHM development.

If synchronization occurs in the system of only two cells with different frequencies, the faster cell dominates the dynamics. Depending on the details of the coupling and contrary to the common conception of excitable systems, a group of slower cells may dominate in linear chains as shown in section 3.2. Still, I observed the dominance of

\footnotetext{
${ }^{1}$ W.-H. Zimmermann, personal communication.
} 
the faster, if not the fastest cells in linear chains of cells with random frequencies to dominate the dynamics at high degrees of synchronization as discussed in section 3.1 and in [33]. This prevalence of faster cells, especially in the case of only two cells agrees with experimental findings of the synchronization behavior of mechanically coupled populations of rat CM [114]. Note that there, the authors couple two populations mechanically among each other while the individual cells within each population are coupled electrically, too. There, the authors even observe phase slips in the slow, driven population that can loosely be connected to the $n: m$ synchronization in my simulations at intermediate elasticities and fluidities of the coupling ECM. There, too, the slower, driven population is not excited at each beat by the faster cell but skips single beats instead. Thus its period, as in the $n: m$ cases I observed, is not constant due to the coupling.

Of course, the simulations can not by any means prove the hypotheses of mechanical synchronization a) happening at all during EHM formation and b) being in fact a necessary condition for successful EHM development. They could, however, have falsified the former hypothesis if they would not have shown synchronization at physiologically relevant frequencies for the qualitative coupling parameters seen in the ECM in experiments. Since in-phase synchronization in two cells and linear chains is observed in the relevant parameter ranges in our simulations, the possible falsification did not happen. Instead I observed synchronization to be most likely in the qualitative regime that proved to be optimal for the successful development of EHM. My findings thus support the hypothesis of the synchronization of CM purely by mechanical and without direct electrical coupling.

The very distinct case of purely elastic coupling shows interesting results on its own, albeit without direct relevance to the study of EHM. Nevertheless, I could show one example of a chimera state with purely local, i.e., only next-neighbor coupling. Although there are other examples of this type of chimera states reported in [115], they are uncommon. The fact that I found antiphase synchronization between the two coherent populations in the chimera state is ultimately a consequence of the fixed boundary conditions I impose on the system. When using periodic boundaries instead I did not observe this antiphase synchrony.

\subsection{Rheology of the extracellular matrix}

In the measurements of the linear properties of the ECM in the presence and absence of cells published in [1], we observed the crucial role of fibroblasts in the stiffening of the ECM. This coincides with findings that fibroblasts have a similar effect on fibrin networks [116]. We also observed that the presence of cardiomyocytes softens the matrix and reduces the stiffening effect of the fibroblasts when both cells are present. The interactions of CM and HFF in matrix formation during EHM growth is apparent in the RNA sequencing data, too. As suggested above, the remodelling of the collagen matrix by fibroblasts may be one reason why they are found to be crucial to the successful growth of EHM [15]. Furthermore we investigated the nonlinear strain-stiffening of 
the ECM in [1]. Interestingly it is most pronounced in the absence of cells. HFF, although stiffening the matrix in the linear regime, weaken the strain-stiffening at larger strains. One possible explanation by Florian Rehfeldt, Susanne Schlick and me for this observation is that HFF compactify the collagen network by exerting a prestrain on the collagen fibers in the network. This leads to the stiffer response of the ECM to small strains. For larger strains, the collagen network itself becomes so stiff, that - when deforming the whole ECM - the deformation of the cells themselves plays the major role in the stress response. Thus the strain-stiffening is softened compared to that of collagen without cells.

In [1] we successfully established an ad-hoc model for the nonlinear stress response of the ECM that reproduced the response for sinusoidal strain input at a frequency of $1 \mathrm{~Hz}$. When attempting to verify the model with nonsinusoidal strain input I experienced difficulties. The model did not correctly predict the measured stress response (cf. figure 4.3b), but, weighing more heavily, I found that the measured stress response itself could be subject to measurement artifacts. Even when measuring the response to a sinusoidal strain with frequencies above $2 \mathrm{~Hz}$, the data was rendered essentially useless by the strong high-frequency fluctuations shown in figure 4.4. Even in communication with the manufacturer of the rheometer, Anton Paar, no solution could be found. Essentially, the ECM as measured here turned out to be soft on or beyond the limits of reliability of the instrument, at least when directly recording the time series of strain, strain rate, and stress. According to the manufacturer this is not specific to the MCR501 rheometer in Florian Rehfeldt's lab but is expected to be common to all commercial shear rheometers; at least to those by Anton Paar. Thus, the ad-hoc model - in fact any model predicting the stress response - could neither be verified nor falsified. Consequently, I am not able to judge whether it is a reasonable extension to the mathematical model of CM coupled mechanically by the ECM. Whether the ad-hoc model indeed provides a realistic description of the mechanical properties of the ECM remains inconclusive.

The alternative of prestress measurements allowed me to probe the nonlinear regime with all control features of the instrument in place, thus possibly increasing the reliability of the rheometer even for my soft samples. Preliminary results seem promising and consistent with sine LAOS measurements but before being able to use these to test the model more data at more frequencies have to be collected. It is also questionable whether prestress measurements are at all a reasonable way of measuring the response of the ECM to the mechanical contraction of CM. The contraction of $\mathrm{CM}$ resembles much more a possibly large deformation from a resting state than a small deformation around a prestrain (or, subsequently, prestress). Still this type of measurements at least offers the perspective of measuring the nonlinear behavior of the ECM at frequencies larger than $1 \mathrm{~Hz}$. An interesting observation in the preliminary prestress measurements is the independence of the response of the frequency of the oscillations. If this holds for a larger range of frequencies it suggests that the stressstrain relation measured for sinusoidal strain at $1 \mathrm{~Hz}$ may be sufficient for finding the model parameters after all. 
Finally I used linear time sweeps of the complex modulus to characterize the gelation of collagen hydrogels prepared using different batches of collagen. Malte Tiburcy's, Susanne Schlick's and my initial hypothesis that these properties could be linked to the performance of EHM produced with the same collagen batches could not be verified. No clear correlation was found ${ }^{2}$. One possible interpretation of this is that the collagen only has to provide a network sufficiently stiff for the fibroblasts to start remodelling. Since they can produce additional collagen [64], they might level out possible initial differences between different batches of collagen.

\subsection{Outlook: Combining simulations and ECM mea- surements}

Since the inclusion of a model describing the nonlinear rheology of the ECM into the numerical model failed in the course of the present thesis, this is a remaining goal for future work. Strain-stiffening increases the range of force transmission in collagen substrates [117] so it will certainly influence the synchronization dynamics of coupled cells. The experimental limitations for direct recording of strain and stress that prevent modeling are likely to remain for any commercial bulk rheometer so a different experimental approach should be used. In preliminary experiments, prestress measurements looked promising, although more data for a larger range of frequencies has to be collected to use the results for modelling. Another possible solution could be using an atomic force microscope (AFM) to measure the stress response of the ECM to deformations of differing amplitudes and rates. In this setting, contraction curves from the numerical model or experiments could be used as input for the motion of the cantilever of the AFM.

Further, to realistically model the situation in early EHM, the mathematical model has to be adapted to actual data from stem-cell-derived cardiomyocytes. Action potential data, e.g., from [15], can be used to determine the action potential time scales $\tau_{x}$ from equations 2.5 and 2.6 in the Mitchell-Schaeffer model. In order to determine the parameters of the mechanical contraction, ideally, measurements of the force of contraction of isolated hiPSC CM are needed. Since they are difficult to obtain, one could try to use the contraction amplitudes found by Sebastian Stein [23] in videos of early EHM recorded by Susanne Schlick and use these data to calculate the force of contraction using the results of the ECM rheology. Determining the parameters of the stretch-activated currents is crucial to quantifying the transition synchronization in coupled cells. Here, a setup using both patch-clamp and, e.g., micropipettes for mechanical stimulation as in [95] or [118] could be used. Detailed studies of the ion channels present in hiPSC CM and their dynamics like [119] could be used to improve the level of detail of the model for the electrical excitation.

The numerical simulations presented in this thesis are right now limited to the study of only two or a linear chain of coupled cells. This should be extended to

\footnotetext{
${ }^{2}$ M. Tiburcy, personal correspondence.
} 
two- or three-dimensional arrays of oscillators, possibly also with irregular spacing. Ultimately, a continuum model allowing for arbitrary geometrical distributions of the cells embedded in the ECM could be devised, even though this would of course dramatically increase the computational effort.

One feature not considered at all in this thesis is the direction of contraction in cardiomyocytes. When growing into functioning tissue, the CM not only synchronize their beating but also have to align their contraction orientations. Nitsan et al. [21] found the orientation to also have an impact on the synchronization behavior of an adult cardiomyocyte. Adding possible interactions between direction of contraction and synchronized beating of the cells to the mathematical model could improve understanding of how and when functioning tissue forms in EHM growth.

\subsection{Conclusion}

During the work on this thesis I investigated numerically the synchronization properties of viscoelastically coupled excitable oscillators as a model for oscillating cardiomyocytes coupled mechanically by a viscoelastic extracellular matrix. Synchronization occurred when the coupling matrix is sufficiently stiff; a finding that agrees well with the experimentally observed time evolution of the ECM. In the numerical simulations, the case of purely elastic coupling, i.e., zero fluidity stood out. It exhibits antiphase synchrony or even antiphase chimera states at frequencies given by the elasticity of the coupling springs and not by the cells anymore.

In rheological experiments, the importance of fibroblasts to the mechanical properties of the ECM could be established. We found nonlinear strain-stiffening behavior already at physiologically relevant strain amplitudes $\gamma_{0} \geq 3 \%$. Because of limitations to the experimental setup when measuring at oscillation frequencies greater than $1 \mathrm{~Hz}$ or with non-sinusoidal strain input, modelling the nonlinear behavior could not be finished and results of the modelling attempts could not be validated experimentally.

Nevertheless I found a qualitative agreement of the parameter ranges that a) allow for synchronization in our simulations and b) are reached by the ECM during the first few hours, especially in the presence of fibroblasts. This may give one explanation for the importance of fibroblasts in EHM growth. My findings thus are a step towards better understanding of EHM development and - when extended by a quantitative description of the ECM rheology by future work - offer a perspective for optimizing the reliability and performance of EHM production. This in turn would improve therapy and drug development for heart failure in the future. 



\section{References}

[1] S. F. Schlick, F. Spreckelsen, M. Tiburcy, L. M. Iyer, T. Meyer, L. C. Zelarayan, S. Luther, U. Parlitz, W.-H. Zimmermann, and F. Rehfeldt. "Agonistic and antagonistic roles of fibroblasts and cardiomyocytes on viscoelastic stiffening of engineered human myocardium". In: Progress in Biophysics and Molecular Biology 144 (July 2019), pp. 51-60. DOI: 10.1016/j.pbiomolbio.2018.11.011.

[2] Y. Kuramoto and D. Battogtokh. "Coexistence of Coherence and Incoherence in Nonlocally Coupled Phase Oscillators". In: Nonlinear Phenomena in Complex Systems 5.4 (2002), pp. 380-385.

[3] D. M. Abrams and S. H. Strogatz. "Chimera States for Coupled Oscillators". In: Physical Review Letters 93.17 (Oct. 2004), p. 174102. DOI: 10.1103/PhysRevLett. 93.174102.

[4] Wikipedia contributors. Heart - Wikipedia, The Free Encyclopedia. https: / / en . wikipedia.org / w / index.php? title =Heart \& oldid $=910050612$. [Online; accessed 23-August-2019]. Aug. 2019.

[5] S. Mendis, P. Puska, B. Norrving, World Health Organization, World Heart Federation, and World Stroke Organization. Global atlas on cardiovascular disease prevention and control. Geneva: World Health Organization, 2011.

[6] E. J. Benjamin et al. "Heart Disease and Stroke Statistics - 2017 Update: A Report From the American Heart Association". In: Circulation 135.10 (Mar. 2017), e146-e603. DOI: 10.1161/CIR.0000000000000485.

[7] D. Berliner and J. Bauersachs. "Current Drug Therapy in Chronic Heart Failure: the New Guidelines of the European Society of Cardiology (ESC)". In: Korean Circulation Journal 47.5 (Sept. 2017), pp. 543-554. DOI: 10.4070/kcj.2017.0030.

[8] P. Ponikowski et al. "2016 ESC Guidelines for the diagnosis and treatment of acute and chronic heart failure". In: European Journal of Heart Failure 18.8 (2016), pp. 891-975. DOI: 10.1002/ejhf.592.

[9] R. J. Johnson, L. L. Bradbury, K. Martin, and J. Neuberger. "Organ Donation and Transplantation in the UK - The Last Decade: A Report From the UK National Transplant Registry". In: Transplantation 97 (Jan. 2014). On behalf of the UK Transplant Registry, S1. DOI: 10.1097/01.TP.0000438215.16737.68.

[10] B. Fujita and W.-H. Zimmermann. "Myocardial Tissue Engineering for Regenerative Applications". In: Current Cardiology Reports 19.9 (Sept. 2017), p. 78. DOI: $10.1007 / \mathrm{s} 11886-017-0892-4$. 
[11] B. Fujita and W.-H. Zimmermann. "Myocardial tissue engineering strategies for heart repair: current state of the art". In: Interactive Cardio Vascular and Thoracic Surgery 1.5 (June 2018). DOI: 10.1093/icvts/ivy208.

[12] W.-H. Zimmermann. "Cardiomyocytes remuscularize the heart". In: Nature Biotechnology 36.7 (July 2018), pp. 592-593. DOI: 10.1038/nbt.4186.

[13] I. Goldfracht et al. "Engineered heart tissue models from hiPSC-derived cardiomyocytes and cardiac ECM for disease modeling and drug testing applications". In: Acta Biomaterialia 92 (July 2019), pp. 145-159. DOI: 10.1016/j.actbio.2019. 05.016 .

[14] S. Schlick. "Fibroblast-Cardiomyocyte Cross-Talk in Heart Muscle Formation and Function". PhD thesis. Göttingen: Georg-August-Universität Göttingen, Feb. 2019.

[15] M. Tiburcy et al. "Defined Engineered Human Myocardium with Advanced Maturation for Applications in Heart Failure Modelling and Repair". In: Circulation 135 (Feb. 2017), pp. 1832-1847. DOI: 10.1161/CIRCULATIONAHA.116.024145.

[16] N. J. Severs. "The cardiac muscle cell". In: BioEssays 22.2 (2000), pp. 188-199. DOI: 10.1002/(SICI)1521-1878(200002)22:2<188::AID-BIES10>3.0.CO;2-T.

[17] G. R. Ferrier and S. E. Howlett. "Cardiac excitation-contraction coupling: role of membrane potential in regulation of contraction". In: American Journal of Physiology - Heart and Circulatory Physiology 280.5 (May 2001), H1928-H1944.

[18] D. M. Bers. "Cardiac excitation-contraction coupling". In: Nature 415.6868 (Jan. 2002), pp. 198-205. DOI: 10.1038/415198a.

[19] S. Rohr. "Role of gap junctions in the propagation of the cardiac action potential". In: Cardiovascular Research 62.2 (May 2004), pp. 309-322. DOI: 10.1016/j. cardiores.2003.11.035.

[20] P. Kohl and F. Sachs. "Mechanoelectric feedback in cardiac cells". In: Philosophical Transactions of the Royal Society of London A: Mathematical, Physical and Engineering Sciences 359.1783 (June 2001), pp. 1173-1185. DOI: 10.1098/ rsta.2001.0824.

[21] I. Nitsan, S. Drori, Y. E. Lewis, S. Cohen, and S. Tzlil. "Mechanical communication in cardiac cell synchronized beating". In: Nature Physics 12.5 (May 2016), pp. 472-477. DOI: 10.1038/nphys3619.

[22] B. Williams and M. Saif. "Phase Dependent Mechanosensitivity in Cardiomyocytes". In: Experimental Mechanics 59.3 (Mar. 2019), pp. 387-393. DOI: 10.1007/s11340-019-00472-9.

[23] S. Stein. "Synchronisation Behaviour of Viscoelastically Coupled Self-Sustained Oscillators as Models for Oscillations of Premature Cardiomyocytes". PhD thesis. Göttingen: Georg-August-Universität Göttingen, Nov. 2017.

[24] A. Pikovsky and M. Rosenblum. "Synchronization". In: Scholarpedia 2.12 (Dec. 2007), p. 1459. DOI: 10.4249/scholarpedia.1459.

[25] J. Peña Ramirez, L. A. Olvera, H. Nijmeijer, and J. Alvarez. "The sympathy of two pendulum clocks: beyond Huygens' observations". In: Scientific Reports 6 (Mar. 2016), p. 23580. DOI: 10.1038/srep23580. 
[26] C. Huygens. Correspondance 1664-1665. Martinus Nijhoff, 1893.

[27] Y. Kuramoto. "Collective synchronization of pulse-coupled oscillators and excitable units". In: Physica D: Nonlinear Phenomena 50.1 (May 1991), pp. 15-30. DOI: 10.1016/0167-2789(91)90075-K.

[28] S. H. Strogatz. "From Kuramoto to Crawford: exploring the onset of synchronization in populations of coupled oscillators". In: Physica D: Nonlinear Phenomena 143.1 (Sept. 2000), pp. 1-20. DOI: 10.1016/S0167-2789(00)00094-4.

[29] F. Dörfler and F. Bullo. "Synchronization in complex networks of phase oscillators: A survey". In: Automatica 50.6 (June 2014), pp. 1539-1564. DOI: 10.1016/j.automatica.2014.04.012.

[30] E. A. Martens, S. Thutupalli, A. Fourrière, and O. Hallatschek. "Chimera states in mechanical oscillator networks". In: Proceedings of the National Academy of Sciences 110.26 (June 2013), pp. 10563-10567. DOI: 10.1073/pnas.1302880110.

[31] B. K. Bera, S. Majhi, D. Ghosh, and M. Perc. "Chimera states: Effects of different coupling topologies". In: EPL (Europhysics Letters) 118.1 (Apr. 2017), p. 10001. DOI: $10.1209 / 0295-5075 / 118 / 10001$.

[32] S. Stein, S. Luther, and U. Parlitz. "Impact of viscoelastic coupling on the synchronization of symmetric and asymmetric self-sustained oscillators". In: New Journal of Physics 19.6 (2017), p. 063040. DoI: 10.1088/1367-2630/aa6d4a.

[33] F. Spreckelsen, S. Luther, and U. Parlitz. "Synchronization of viscoelastically coupled excitable oscillators". In: Physical Review E 100.3 (Sept. 2019), p. 032214. DOI: 10.1103/PhysRevE.100.032214.

[34] Wikipedia contributors. Cardiac physiology - Wikipedia, The Free Encyclopedia. https: / / en.wikipedia.org/w/index.php?title $=$ Cardiac_physiology\&oldid $=$ 863301422. [Online; accessed 13-September-2019]. 2018.

[35] J. G. Betts, P. Desaix, E. Johnson, J. E. Johnson, O. Korol, D. Kruse, B. Poe, J. A. Wise, M. Womble, and K. A. Young. Anatomy $\&$ Physiology. AP-2013003(03/17)-LC. Houston, Texas: OpenStax, 2017.

[36] R. D. Ponti, S. Y. Ho, J. A. Salerno-Uriarte, M. Tritto, and G. Spadacini. "Electroanatomic Analysis of Sinus Impulse Propagation in Normal Human Atria". In: Journal of Cardiovascular Electrophysiology 13.1 (2002), pp. 1-10. DOI: 10.1046/j.1540-8167.2002.00001.x.

[37] National Heart, Lung, and Blood Institute (NHLBI). Arrhythmia. https://www. nhlbi.nih.gov/health-topics/arrhythmia. [Online; accessed 24-September-2019].

[38] S. V. Pandit and J. Jalife. "Rotors and the Dynamics of Cardiac Fibrillation". In: Circulation Research 112.5 (Mar. 2013), pp. 849-862. DOI: 10.1161/ CIRCRESAHA.111.300158.

[39] World Health Organization (WHO). Cardiovascular diseases (CVDs) Fact Sheet. https://www.who.int/news-room/fact-sheets/detail/cardiovascular-diseases(cvds). [Online; accessed 24-September-2019].

[40] T. Neumann, J. Biermann, A. Neumann, J. Wasem, G. Ertl, R. Dietz, and R. Erbel. "Herzinsuffizienz - Häufigster Grund für Krankenhausaufenthalte Medizinische und ökonomische Aspekte". In: Deutsches Ärzteblatt International 106.16 (Apr. 2009), pp. 269-275. DOI: 10.3238/arztebl.2009.0269. 
[41] J. G. Travers, F. A. Kamal, J. Robbins, K. E. Yutzey, and B. C. Blaxall. "Cardiac Fibrosis". In: Circulation Research 118.6 (Mar. 2016), pp. 1021-1040. DOI: 10.1161/CIRCRESAHA.115.306565.

[42] B. Fujita, M. Tiburcy, S. Ensminger, and W.-H. Zimmermann. "State-of-the-Art in Tissue-Engineered Heart Repair". In: Cardiac Regeneration. Cardiac and Vascular Biology. Springer, Cham, 2017, pp. 219-239. DOI: 10.1007/978-3-31956106-6_10.

[43] B. Fujita and W.-H. Zimmermann. "Engineered Heart Repair". In: Clinical Pharmacology \&6 Therapeutics 102.2 (2017), pp. 197-199. DOI: 10.1002/cpt.724.

[44] Wikipedia contributors. Cardiac muscle - Wikipedia, The Free Encyclopedia. https : / / en . wikipedia . org / w / index . php? title =Cardiac_muscle \& oldid $=$ 917317906. [Online; accessed 25-September-2019]. 2019.

[45] W. E. Louch, J. Hake, H. K. Mørk, K. Hougen, B. Skrbic, D. Ursu, T. Tønnessen, I. Sjaastad, and O. M. Sejersted. "Slow Ca2+ sparks de-synchronize Ca2+ release in failing cardiomyocytes: Evidence for altered configuration of $\mathrm{Ca} 2+$ release units?" In: Journal of Molecular and Cellular Cardiology. Calcium Signaling in Heart 58 (May 2013), pp. 41-52. DOI: 10.1016/j.yjmcc.2013.01.014.

[46] V. Talman and H. Ruskoaho. "Cardiac fibrosis in myocardial infarction-from repair and remodeling to regeneration". In: Cell and Tissue Research 365.3 (Sept. 2016), pp. 563-581. DOI: 10.1007/s00441-016-2431-9.

[47] H. Antoni. "Erregungsphysiologie des Herzens". In: Physiologie des Menschen. Ed. by R. F. Schmidt, G. Thews, and F. Lang. Springer-Lehrbuch. Berlin, Heidelberg: Springer, 2000, pp. 472-497. DOI: 10.1007/978-3-662-09346-7_23.

[48] A. O. Grant. "Cardiac Ion Channels". In: Circulation: Arrhythmia and Electrophysiology 2.2 (Apr. 2009), pp. 185-194. DOI: 10.1161/CIRCEP.108.789081.

[49] V. S. Zykov. "Excitable media". In: Scholarpedia 3.5 (May 2008), p. 1834. DoI: 10.4249/scholarpedia.1834.

[50] H. Hu and F. Sachs. "Stretch-Activated Ion Channels in the Heart". In: Journal of Molecular and Cellular Cardiology 29.6 (June 1997), pp. 1511-1523. DOI: 10.1006/jmcc.1997.0392.

[51] P. Kohl, P. Hunter, and D. Noble. "Stretch-induced changes in heart rate and rhythm: clinical observations, experiments and mathematical models". In: Progress in Biophysics and Molecular Biology 71.1 (Jan. 1999), pp. 91-138. DOI: 10.1016/S0079-6107(98)00038-8.

[52] Wikipedia contributors. Sarcomere - Wikipedia, The Free Encyclopedia. https: //en.wikipedia.org/w/index.php?title=Sarcomere\&oldid=914631591. [Online; accessed 26-September-2019]. 2019.

[53] D. M. Bers. "Calcium Cycling and Signaling in Cardiac Myocytes". In: Annual Review of Physiology 70.1 (2008), pp. 23-49. DOI: 10.1146/annurev.physiol.70. 113006.100455.

[54] T. Eschenhagen et al. "Three-dimensional reconstitution of embryonic cardiomyocytes in a collagen matrix: a new heart muscle model system." In: The FASEB Journal 11.8 (Jan. 1997), pp. 683-694. 
[55] W. H. Zimmermann, C. Fink, D. Kralisch, U. Remmers, J. Weil, and T. Eschenhagen. "Three-dimensional engineered heart tissue from neonatal rat cardiac myocytes". In: Biotechnology and Bioengineering 68.1 (2000), pp. 106-114. DOI: 10.1002/(SICI)1097-0290(20000405)68:1<106::AID-BIT13>3.0.CO;2-3.

[56] W.-H. Zimmermann, M. Didié, G. H. Wasmeier, U. Nixdorff, A. Hess, I. Melnychenko, O. Boy, W. L. Neuhuber, M. Weyand, and T. Eschenhagen. "Cardiac Grafting of Engineered Heart Tissue in Syngenic Rats". In: Circulation 106.12 suppl 1 (Sept. 2002), pp. I-151-I-157. DOI: 10.1161/01.cir.0000032876.55215.10.

[57] M. Tiburcy, T. Meyer, P. L. Soong, and W.-H. Zimmermann. "Collagen-Based Engineered Heart Muscle". In: Cardiac Tissue Engineering: Methods and Protocols. Ed. by M. Radisic and L. D. Black III. Methods in Molecular Biology. New York, NY: Springer New York, 2014, pp. 167-176. DOI: 10.1007/978-1-49391047-2 15.

[58] A. Hansen, A. Eder, M. Bönstrup, M. Flato, M. Mewe, S. Schaaf, B. Aksehirlioglu, A. Schwoerer, J. Uebeler, and T. Eschenhagen. "Development of a Drug Screening Platform Based on Engineered Heart Tissue". In: Circulation Research 107.1 (July 2010), pp. 35-44. DOI: 10.1161/CIRCRESAHA.109.211458.

[59] K. Matsuura, Y. Haraguchi, T. Shimizu, and T. Okano. "Cell sheet transplantation for heart tissue repair". In: Journal of Controlled Release. Second Symposium on Innovative Polymers for Controlled Delivery (SIPCD 2012) 169.3 (Aug. 2013), pp. 336-340. DOI: 10.1016/j.jconrel.2013.03.003.

[60] M. Kawamura et al. "Enhanced Therapeutic Effects of Human iPS Cell DerivedCardiomyocyte by Combined Cell-Sheets with Omental Flap Technique in Porcine Ischemic Cardiomyopathy Model". In: Scientific Reports 7.1 (Aug. 2017), p. 8824. DOI: 10.1038/s41598-017-08869-z.

[61] S. A. Doppler, M.-A. Deutsch, R. Lange, and M. Krane. "Cardiac regeneration: current therapies - future concepts". In: Journal of Thoracic Disease 5.5 (Oct. 2013), pp. 683-697. DOI: 10.3978/j.issn.2072-1439.2013.08.71.

[62] J. J. H. Chong et al. "Human embryonic-stem-cell-derived cardiomyocytes regenerate non-human primate hearts". In: Nature 510.7504 (June 2014), pp. 273277. DOI: $10.1038 /$ nature13233.

[63] K. Miyamoto et al. "Direct In Vivo Reprogramming with Sendai Virus Vectors Improves Cardiac Function after Myocardial Infarction". In: Cell Stem Cell 22.1 (Jan. 2018), 91-103.e5. DOI: 10.1016/j.stem.2017.11.010.

[64] S. Ricard-Blum. "The Collagen Family". In: Cold Spring Harbor Perspectives in Biology 3.1 (Jan. 2011), a004978. DOI: 10.1101/cshperspect.a004978.

[65] M. D. Shoulders and R. T. Raines. "Collagen Structure and Stability". In: Annual Review of Biochemistry 78.1 (2009), pp. 929-958. DOI: 10.1146/annurev. biochem.77.032207.120833.

[66] Wikipedia contributors. Collagen - Wikipedia, The Free Encyclopedia. https: //en.wikipedia.org/w/index.php?title=Collagen\&oldid=919623249. [Online; accessed 9-October-2019]. 2019. 
[67] K. A. Jansen, A. J. Licup, A. Sharma, R. Rens, F. C. MacKintosh, and G. H. Koenderink. "The Role of Network Architecture in Collagen Mechanics". In: Biophysical Journal 114.11 (June 2018), pp. 2665-2678. DOI: 10.1016/j.bpj.2018. 04.043.

[68] J. P. R. O. Orgel, T. C. Irving, A. Miller, and T. J. Wess. "Microfibrillar structure of type I collagen in situ". In: Proceedings of the National Academy of Sciences 103.24 (June 2006), pp. 9001-9005. DOI: 10.1073/pnas.0502718103.

[69] N. S. Murthy. "Liquid crystallinity in collagen solutions and magnetic orientation of collagen fibrils". In: Biopolymers 23.7 (1984), pp. 1261-1267. DOI: 10.1002/ bip.360230710.

[70] Y. Li, A. Asadi, M. R. Monroe, and E. P. Douglas. "pH effects on collagen fibrillogenesis in vitro: Electrostatic interactions and phosphate binding". In: Materials Science and Engineering: C 29.5 (June 2009), pp. 1643-1649. DOI: 10.1016/j.msec.2009.01.001.

[71] J. W. S. Rayleigh. The theory of sound. London, 1894.

[72] L. Kocarev and U. Parlitz. "General Approach for Chaotic Synchronization with Applications to Communication". In: Physical Review Letters 74.25 (June 1995), pp. 5028-5031. DOI: 10.1103/PhysRevLett.74.5028.

[73] J. A. Acebrón, L. L. Bonilla, C. J. Pérez Vicente, F. Ritort, and R. Spigler. "The Kuramoto model: A simple paradigm for synchronization phenomena". In: Reviews of Modern Physics 77.1 (Apr. 2005), pp. 137-185. DOI: 10.1103/ RevModPhys.77.137.

[74] A. Pikovsky, M. Rosenblum, and J. Kurths. Synchronization - A Universal Concept in Nonlinear Sciences. Cambridge: Cambridge University Press, Oct. 2001. DOI: $10.1017 / \mathrm{CBO} 9780511755743$.

[75] A. Balanov, N. Janson, D. Postnov, and O. Sosnovtseva. Synchronization - From Simple to Complex. Springer Series in Synergetics. Springer, Berlin, Heidelberg, 2009. DOI: $10.1007 / 978-3-540-72128-4$.

[76] Y. Kuramoto. "Mutual Entrainment". In: Chemical Oscillations, Waves, and Turbulence. Ed. by Y. Kuramoto. Springer Series in Synergetics. Berlin, Heidelberg: Springer Berlin Heidelberg, 1984, pp. 60-88. DOI: 10.1007/978-3-64269689-3 5.

[77] F. P. Kemeth, S. W. Haugland, L. Schmidt, I. G. Kevrekidis, and K. Krischer. "A classification scheme for chimera states". In: Chaos: An Interdisciplinary Journal of Nonlinear Science 26.9 (Aug. 2016), p. 094815. DOI: 10.1063/1.4959804.

[78] R. S. Campos, M. Lobosco, and R. W. d. Santos. "A GPU-based heart simulator with mass-spring systems and cellular automaton". In: The Journal of Supercomputing 69.1 (May 2014), pp. 1-8. DOI: 10.1007/s11227-014-1199-5.

[79] D. Barkley. "A model for fast computer simulation of waves in excitable media". In: Physica D: Nonlinear Phenomena 49.1 (Apr. 1991), pp. 61-70. DOI: 10.1016/ 0167-2789(91)90194-E.

[80] R. R. Aliev and A. V. Panfilov. "A simple two-variable model of cardiac excitation". In: Chaos, Solitons 83 Fractals 7.3 (Mar. 1996), pp. 293-301. DOI: 10.1016/0960-0779(95)00089-5. 
[81] F. Fenton and A. Karma. "Vortex dynamics in three-dimensional continuous myocardium with fiber rotation: Filament instability and fibrillation". In: Chaos: An Interdisciplinary Journal of Nonlinear Science 8.1 (Mar. 1998), pp. 20-47. DOI: $10.1063 / 1.166311$.

[82] K. H. W. J. ten Tusscher, D. Noble, P. J. Noble, and A. V. Panfilov. "A model for human ventricular tissue". In: American Journal of Physiology-Heart and Circulatory Physiology 286.4 (Apr. 2004), H1573-H1589. DOI: 10.1152/ajpheart. 00794.2003.

[83] V. E. Bondarenko, G. P. Szigeti, G. C. L. Bett, S.-J. Kim, and R. L. Rasmusson. "Computer model of action potential of mouse ventricular myocytes". In: American Journal of Physiology-Heart and Circulatory Physiology 287.3 (Sept. 2004), H1378-H1403. DOI: 10.1152/ajpheart.00185.2003.

[84] A. Mahajan et al. "A Rabbit Ventricular Action Potential Model Replicating Cardiac Dynamics at Rapid Heart Rates". In: Biophysical Journal 94.2 (Jan. 2008), pp. 392-410. DOI: 10.1529/biophysj.106.98160.

[85] C. C. Mitchell and D. G. Schaeffer. "A two-current model for the dynamics of cardiac membrane". In: Bulletin of Mathematical Biology 65.5 (Sept. 2003), pp. 767-793. DOI: 10.1016/S0092-8240(03)00041-7.

[86] K. Djabella, M. Landau, and M. Sorine. "A two-variable model of cardiac action potential with controlled pacemaker activity and ionic current interpretation". In: 2007 46th IEEE Conference on Decision and Control. Dec. 2007, pp. 51865191. DOI: 10.1109/CDC.2007.4434970.

[87] A. Karma. "Electrical alternans and spiral wave breakup in cardiac tissue". In: Chaos: An Interdisciplinary Journal of Nonlinear Science 4.3 (Sept. 1994), pp. 461-472. DOI: 10.1063/1.166024.

[88] P. J. Hunter, A. D. McCulloch, and H. E. D. J. ter Keurs. "Modelling the mechanical properties of cardiac muscle". In: Progress in Biophysics and Molecular Biology 69.2 (Mar. 1998), pp. 289-331. DOI: 10.1016/S0079-6107(98)00013-3.

[89] L. D. Weise and A. V. Panfilov. "A Discrete Electromechanical Model for Human Cardiac Tissue: Effects of Stretch-Activated Currents and Stretch Conditions on Restitution Properties and Spiral Wave Dynamics". In: PLOS ONE 8.3 (Mar. 2013), e59317. DOI: 10.1371/journal.pone.0059317.

[90] L. D. Weise, M. P. Nash, and A. V. Panfilov. "A Discrete Model to Study Reaction-Diffusion-Mechanics Systems". In: PLOS ONE 6.7 (July 2011), e21934. DOI: 10.1371/journal.pone.0021934.

[91] L. D. Weise and A. V. Panfilov. "Emergence of Spiral Wave Activity in a Mechanically Heterogeneous Reaction-Diffusion-Mechanics System". In: Physical Review Letters 108.22 (June 2012), p. 228104. DOI: 10.1103/PhysRevLett.108. 228104.

[92] F. Sachs. "Modeling Mechanical-Electrical Transduction in the Heart". In: Cell Mechanics and Cellular Engineering. Springer, New York, NY, 1994, pp. 308328. DOI: $10.1007 / 978-1-4613-8425-0 \_18$. 
[93] O. Cohen and S. A. Safran. "Theory of frequency response of mechanically driven cardiomyocytes". In: Scientific Reports 8.1 (Feb. 2018), p. 2237. DOI: 10.1038/s41598-018-20307-2.

[94] M. P. Nash and A. V. Panfilov. "Electromechanical model of excitable tissue to study reentrant cardiac arrhythmias". In: Progress in Biophysics and Molecular Biology. Modelling Cellular and Tissue Function 85.2-3 (June 2004), pp. 501-522. DOI: 10.1016/j.pbiomolbio.2004.01.016.

[95] Y. H. Zhang, J. B. Youm, H. K. Sung, S. H. Lee, S. Y. Ryu, S.-H. Lee, W.-K. Ho, and Y. E. Earm. "Stretch-activated and background non-selective cation channels in rat atrial myocytes". In: The Journal of Physiology 523.3 (Mar. 2000), pp. 607-619. DOI: 10.1111/j.1469-7793.2000.00607.x.

[96] C. Storm, J. J. Pastore, F. C. MacKintosh, T. C. Lubensky, and P. A. Janmey. "Nonlinear elasticity in biological gels". In: Nature 435.7039 (May 2005), pp. 191194. DOI: $10.1038 /$ nature03521.

[97] N. A. Kurniawan, L. H. Wong, and R. Rajagopalan. "Early Stiffening and Softening of Collagen: Interplay of Deformation Mechanisms in Biopolymer Networks". In: Biomacromolecules 13.3 (Mar. 2012), pp. 691-698. DOI: 10.1021/ bm2015812.

[98] N. W. Tschoegl. The Phenomenological Theory of Linear Viscoelastic Behavior. Berlin, Heidelberg: Springer Berlin Heidelberg, 1989. DOI: 10.1007/978-3-64273602-5.

[99] G. van Rossum. Python tutorial. Tech. rep. CS-R9526. Amsterdam: Centrum voor Wiskunde en Informatica (CWI), May 1995.

[100] S. Behnel, R. Bradshaw, C. Citro, L. Dalcin, D. S. Seljebotn, and K. Smith. "Cython: The Best of Both Worlds". In: Computing in Science Engineering 13.2 (Mar. 2011), pp. 31-39. DOI: 10.1109/MCSE.2010.118.

[101] E. Jones, T. Oliphant, P. Peterson, et al. SciPy: Open source scientific tools for Python. http://www.scipy.org/. [Online; accessed 20-September-2019]. 2001.

[102] A. N. Iyer and R. A. Gray. "An Experimentalist's Approach to Accurate Localization of Phase Singularities during Reentry". In: Annals of Biomedical Engineering 29.1 (Jan. 2001), pp. 47-59. DOI: 10.1114/1.1335538.

[103] M.-A. Bray and J. P. Wikswo. "Considerations in phase plane analysis for nonstationary reentrant cardiac behavior". In: Physical Review E 65.5 (May 2002), p. 051902. DOI: 10.1103/PhysRevE.65.051902.

[104] R. H. Clayton, E. A. Zhuchkova, and A. V. Panfilov. "Phase singularities and filaments: Simplifying complexity in computational models of ventricular fibrillation". In: Progress in Biophysics and Molecular Biology. From Funny Current to Current Physiome 90.1 (Jan. 2006), pp. 378-398. DOI: 10.1016/j. pbiomolbio.2005.06.011.

[105] A. J. Giacomin, P. H. Gilbert, D. Merger, and M. Wilhelm. "Large-amplitude oscillatory shear: comparing parallel-disk with cone-plate flow". In: Rheologica Acta 54.4 (Apr. 2015), pp. 263-285. DOI: 10.1007/s00397-014-0819-6. 
[106] R. H. Ewoldt, A. E. Hosoi, and G. H. McKinley. "New measures for characterizing nonlinear viscoelasticity in large amplitude oscillatory shear". In: Journal of Rheology 52.6 (Nov. 2008), pp. 1427-1458. DOI: 10.1122/1.2970095.

[107] K. Hyun, M. Wilhelm, C. O. Klein, K. S. Cho, J. G. Nam, K. H. Ahn, S. J. Lee, R. H. Ewoldt, and G. H. McKinley. "A review of nonlinear oscillatory shear tests: Analysis and application of large amplitude oscillatory shear (LAOS)". In: Progress in Polymer Science 36.12 (Dec. 2011), pp. 1697-1753. DOI: 10.1016/j. progpolymsci.2011.02.002.

[108] D. M. Heyes. "Shear thinning and thickening of the Lennard-Jones liquid. A molecular dynamics study". In: Journal of the Chemical Society, Faraday Transactions 2: Molecular and Chemical Physics 82.9 (1986), pp. 1365-1383. DOI: $10.1039 /$ F29868201365.

[109] Wikipedia contributors. Chebyshev polynomials - Wikipedia, The Free Encyclopedia. https://en.wikipedia.org/w/index.php?title=Chebyshev_polynomials\& oldid $=921602451$. [Online; accessed 22-October-2019]. 2019.

[110] S. W. Katicha and G. W. Flintsch. "Fractional viscoelastic models: master curve construction, interconversion, and numerical approximation". In: Rheologica Acta 51.8 (Aug. 2012), pp. 675-689. DOI: 10.1007/s00397-012-0625-y.

[111] J. E. Marsden and T. J. R. Hughes. Mathematical Foundations of Elasticity. New York: Dover Publications, 2015.

[112] G. A. Holzapfel and R. W. Ogden. "Constitutive modelling of passive myocardium: a structurally based framework for material characterization". In: Philosophical Transactions of the Royal Society of London A: Mathematical, Physical and Engineering Sciences 367.1902 (Sept. 2009), pp. 3445-3475. DOI: 10.1098/rsta.2009.0091.

[113] Wikipedia contributors. Reverse pipetting - Wikipedia, The Free Encyclopedia. https: / / en.wikipedia.org / w / index.php?title=Reverse_pipetting \& oldid $=$ 799100465. [Online; accessed 25-January-2018]. 2017.

[114] B. J. Williams and M. T. A. Saif. "Induced Coupling Between Contractile Cells". In: Mechanics of Biological Systems and Materials, Volume 6. Ed. by S. A. Tekalur, P. Zavattieri, and C. S. Korach. Conference Proceedings of the Society for Experimental Mechanics Series. Springer International Publishing, 2016, pp. 91-97.

[115] C. R. Laing. "Chimeras in networks with purely local coupling". In: Physical Review E 92.5 (Nov. 2015), p. 050904. DOI: 10.1103/PhysRevE.92.050904.

[116] K. A. Jansen, R. G. Bacabac, I. K. Piechocka, and G. H. Koenderink. "Cells Actively Stiffen Fibrin Networks by Generating Contractile Stress". In: Biophysical Journal 105.10 (Nov. 2013), pp. 2240-2251. DOI: 10.1016/j.bpj.2013.10.008.

[117] M. S. Hall, F. Alisafaei, E. Ban, X. Feng, C.-Y. Hui, V. B. Shenoy, and M. Wu. "Fibrous nonlinear elasticity enables positive mechanical feedback between cells and ECMs". In: Proceedings of the National Academy of Sciences 113.49 (June 2016), pp. 14043-14048. DOI: 10.1073/pnas.1613058113. 
[118] T. Zeng, G. C. L. Bett, and F. Sachs. "Stretch-activated whole cell currents in adult rat cardiac myocytes". In: American Journal of Physiology - Heart and Circulatory Physiology 278.2 (Feb. 2000), H548-H557.

[119] Z. Zhao et al. "Ion Channel Expression and Characterization in Human Induced Pluripotent Stem Cell-Derived Cardiomyocytes". In: Stem Cells International 2018 (Jan. 2018). DOI: 10.1155/2018/6067096. 


\section{Appendix A}

\section{Initial conditions and parameters}

Most parameters of the mathematical model for the viscoelastically coupled excitable oscillators were either taken from the original publications of the individual constituents or - in the case of the passive restoring force - adapted once to obtain reasonable contraction curves (cf. figure 2.2b). The coupling parameters were scanned and did not have to be fixed to single values. A parameter that needed a little more care is $a$ in equation 2.11 used to tune the frequency of oscillation.

When choosing the initial conditions for the integration of the model, one has to be careful about the initial phases. Since the phase of oscillation is determined post-hoc from the time series by linear interpolation between two consecutive maxima in $v(t)$ as described in section 2.2.2, it is not clear how an initial phase should be defined.

In the following I explain how the parameter $a$ is found for a specific frequency and how the initial phases and thus the initial conditions for the cells are defined.

\section{A.1 Natural frequencies of individual cells}

As discussed in section 2.1.1, the oscillations of the dynamics of the membrane voltage $v$ are controlled by the single parameter $a$ in the modified Mitchell-Schaeffer model in equation 2.11. Oscillations occur for $a \geq 0.0126$ (see section 3.1 or [33] and figure 1 therein). Then a frequency of oscillation $\nu(a)$ can be obtained by integrating equations 2.11 and 2.12 for a specific $a$ and, as explained in section 2.2.1, determine the period of oscillation by calculating the time interval between two consecutive $v=0.5$ crossings in upwards direction. If one now wants to have a cell oscillating at a specific frequency $\nu^{*}$, one has to find the corresponding $a^{*}$. This is done by numerically finding the root of

$$
C(a)=\nu(a)-\nu^{*}
$$

$\nu(a)$ is found by integrating equations 2.11 and 2.12 over a period of $11 \mathrm{~s}$ and cutting off the first $1 \mathrm{~s}$ to get rid of transients. From this, as explained above, the period is calculated from which the frequency is obtained. Roots of $C(a)$ then are found by 
using Newton's method as implemented in the optimize.newton function from the SciPy [101] library. A candidate $a$ is accepted as $a^{*}$ if

$$
\frac{\left|C\left(a^{*}\right)\right|}{\nu^{*}} \leq 1 \times 10^{-4}
$$

\section{A.2 Initial phases and initial conditions}

When calculating initial phases of a cell with a given frequency, I started out with the time series $v(t), h(t)$ of an isolated cell with that frequency. They are obtained by integrating equations 2.11 and 2.12 over at least one full period of the cell. From $v(t)$, the phase $\psi(t)$ is calculated as explained in section 2.2.2. Let $\psi_{\text {init }}$ be the desired initial phase of the cell, e.g., from the random initial phase distribution used for the linear chains in section 3.1. From $\psi(t)$, find $t_{\text {init }}$ such that $\psi\left(t_{\text {init }}\right)=\psi_{\text {init }}$. Then, $v\left(t_{\text {init }}\right), h\left(t_{\text {init }}\right)$ are used as initial conditions for the cell. 


\section{Appendix B}

\section{Ageing of Collagen}

During the course of the experiments, we observed not only differences between different batches of collagen but also that the hydrogels deteriorated over time. As in the case of comparing different batches of collagen in section 4.3, I will limit the considerations to time sweeps of the storage modulus $G^{\prime}$ here. The loss modulus $G^{\prime \prime}$ showed a qualitatively similar behavior to $G^{\prime}$. As explained in section 4.3, we first noticed the deterioration of a collagen batch in preliminary experiments to [1] with collagen batch 15CSA02 in January and February 2018 when gelation would not even occur strong enough for the cell dependent remodeling to set in. The - at that time - fresh (see table B.1) batch 17CSA03 did not display these problems and polymerized faster and stronger. When recording similar time sweeps of 17CSA03 in September 2018, however, both the speed and the final plateau of gelation in time sweeps of $G^{\prime}$ were reduced compared to measurements in early 2018 as shown in figure B.1. This can only partially be attributed to the change of protocol for the collagen comparison in fall 2018, because measurements with the EHM protocol in September 2018 showed a similar decrease in $G^{\prime}$ compared to March 2018. Between fall 2018 and the following series of experiments in June and July 2019, the average plateau value of $G^{\prime}$ decreased further while the speed of gelation remained comparable as can be seen in figure B.1b. Here, the PBS

Table B.1 Production dates of the collagen batches used for experiments in this thesis. Data provided by M. Tiburcy, UMG.

\begin{tabular}{|c|c|c|}
\hline Batch & Concentration $[\mathrm{mg} / \mathrm{ml}]$ & Date \\
\hline 14CSA02 & 6.56 & $2015-05-13$ \\
15CSA02 & 6.4 & $2015-08-05$ \\
17CSA03 & 6.49 & $2017-12-21$ \\
18CB023001 & 6.49 & $2017-12-21$ \\
17CB024002 & 6.72 & $2018-06-11$ \\
17CB024001 & 6.91 & $2018-06-14$ \\
18CB024001 & 6.81 & $2018-06-11$ \\
18CB024003 & 6.84 & $2018-09-08$ \\
\hline
\end{tabular}




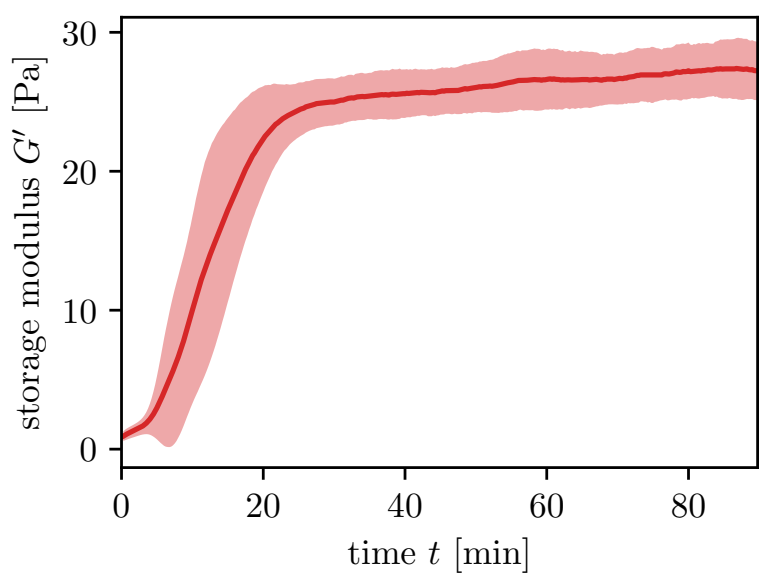

(a) March 2018, EHM protocol

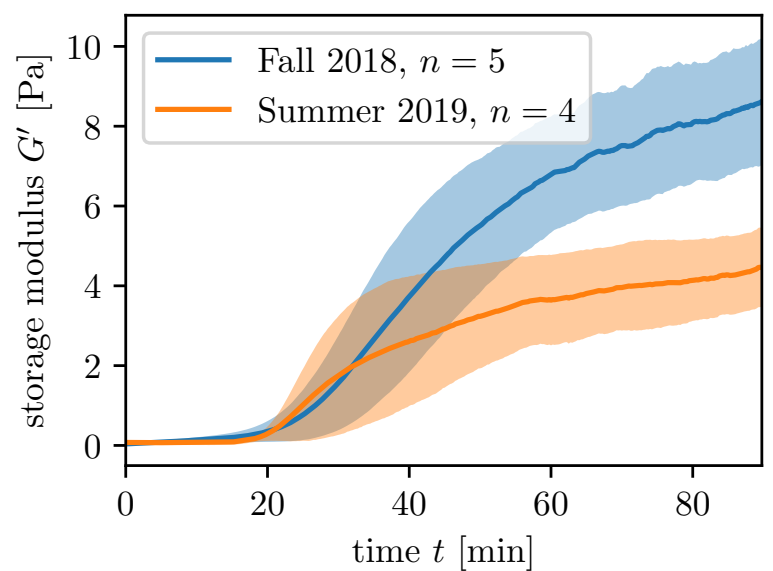

(b) PBS protocol

Figure B.1 Average storage modulus $G^{\prime}$ of collagen batch 17CSA03 with the EHM protocol (a) (same data as in [1] and in figure 4.6) and with the PBS protocol (b). The shaded regions denote the standard deviations. "Fall 2018" denotes experiments conducted from September to November 2018, "Summer 2019" experiments from June and July 2019. The decrease in complex modulus from March to fall 2018 may be caused by the different protocols, although measurements with the EHM protocol in September 2018 showed similar $G^{\prime}$ as the PBS protocol (data not shown). $G^{\prime}$ decreases further from fall 2018 to summer 2019.

protocol was used in both cases so a change in protocol cannot explain the differences.

The same observation can be made for the gelation characteristics, plateau $G^{\prime}$, gelation strength, and time of half-plateau $t_{\mathrm{MH}}$ (see figure B.2a) of all batches that were used in both series, i.e., in fall 2018 and summer 2019. The averages of the three quantities are shown figures B.2b to B.2d. While a decrease in plateau and gelation strength is apparent for all batches, the polymerization times as indicated by $t_{\mathrm{H}}$ remained comparable. For the much older collagen batches 14CSA02 and 15CSA02 (cf. table B.1), no polymerization could be observed in further experiments in December 2018. 


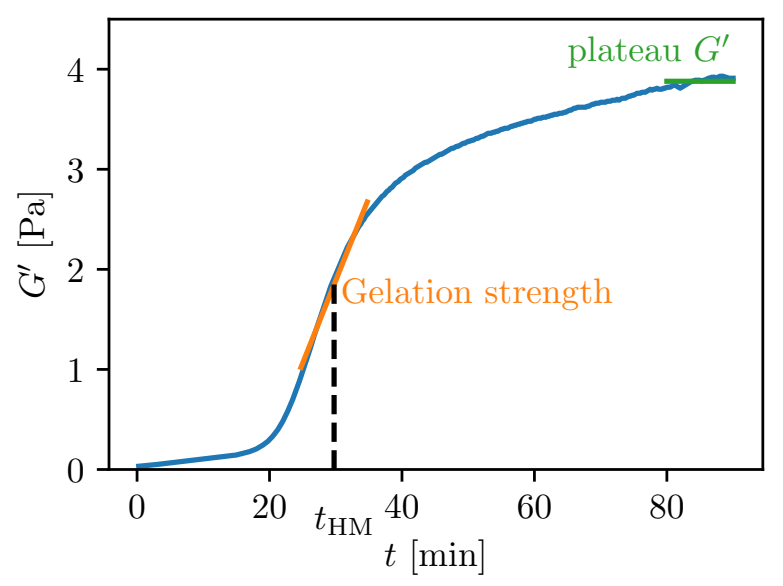

(a) Relevant quantities

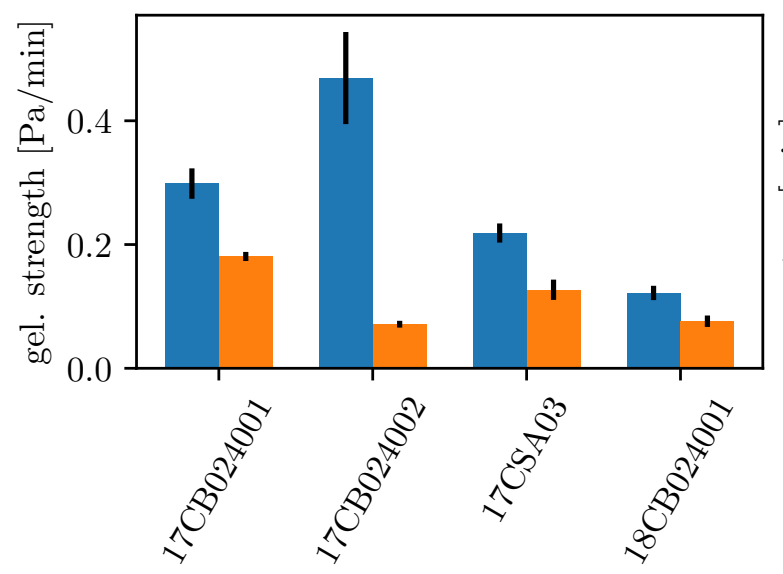

(c) Gelation strength

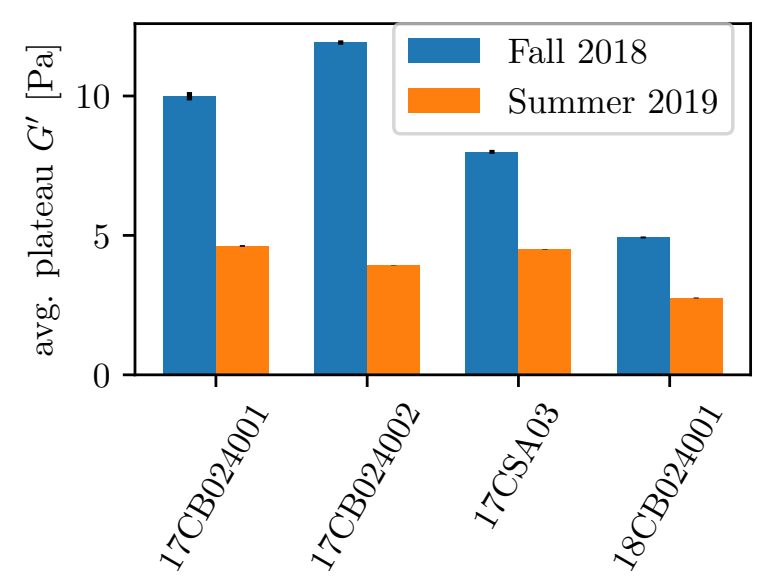

(b) Plateau $G^{\prime}$

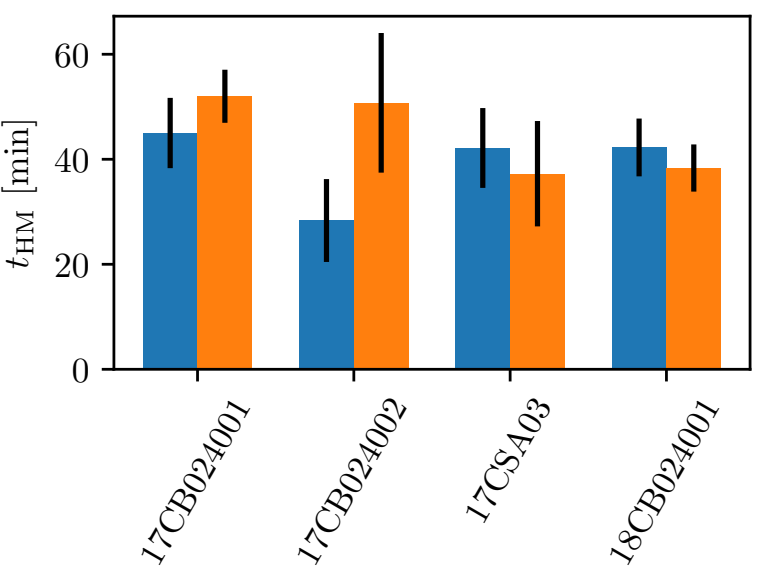

(d) $t_{\mathrm{HM}}$

Figure B.2 Average plateau $G^{\prime}$, gelation strength, and time at half plateau $t_{\mathrm{HM}}$. The relevant quantities are indicated ate the time sweep of $G^{\prime}$ in (a). Plateau $G^{\prime}$ is the average of $G^{\prime}$ over the last $10 \mathrm{~min}$ of the time sweep, $t_{\mathrm{HM}}$ is the point in time when $G^{\prime}$ reaches the value of half the plateau and the gelation strength is the slope of $G^{\prime}$ at $t=t_{\mathrm{HM}}$. (b) - (c) show the respective quantities for all batches in the experiments in fall 2018 and summer 2019. Error bars indicate standard deviation. Plateau modulus and gelation strength decrease in all batches over time while $t_{\mathrm{HM}}$ remains comparable. In most cases, each data point corresponds to the average of three measurements, except for 17CSA03 with $n=5$ in fall 2018 and $n=4$ in summer 2019. 



\section{Appendix C}

\section{Coefficients of the ad-hoc model}

\section{C.1 Supplementary material of Schlick et al. 2019}

The following table C.1 is reproduced from the supplementary material of

S. F. Schlick, F. Spreckelsen, M. Tiburcy, L. M. Iyer, T. Meyer, L. C. Zelarayan, S. Luther, U. Parlitz, W.-H. Zimmermann, and F. Rehfeldt.

"Agonistic and antagonistic roles of fibroblasts and cardiomyocytes on viscoelastic stiffening of engineered human myocardium". In: Progress in Biophysics and Molecular Biology 144 (July 2019), pp. 51-60. DOI: 10.1016/j.pbiomolbio.2018.11.011

published under the Creative Commons Attribution License (CC BY 4.0).

Table C.1 Supplementary table 3 containing the coefficients found via basin-hopping optimization for the ad-hoc model curves in figure 7 of [1].

\begin{tabular}{|l|c|c|c|c|}
\hline Parameters & Collagen & CM & Fib & EHM \\
\hline$c_{1}$ & 34.65 & -16.47 & 64.91 & 5.46 \\
\hline$c_{2}$ & 161.1 & 10.31 & 9.18 & 21.15 \\
\hline$c_{3}$ & -918.1 & -33.61 & 9534 & -0.03 \\
\hline$c_{4}$ & 1106 & -95.56 & -481.6 & -730.1 \\
\hline$c_{5}$ & 576.4 & -1.51 & 6.72 & -3.15 \\
\hline$c_{6}$ & 768.5 & 680.3 & 492.4 & 564.8 \\
\hline$c_{7}$ & 4.59 & -2.04 & 0.59 & 22.42 \\
\hline$c_{8}$ & -625.8 & -9.37 & 0.46 & -57.05 \\
\hline$c_{9}$ & -0.14 & -0.23 & -0.12 & -0.46 \\
\hline
\end{tabular}


Table C.2 Coefficients of the ad-hoc model found by basin hopping optimization to the stress response of sinusoidal strain input with amplitudes from $1 \%$ to $10 \%$ at $1 \mathrm{~Hz}$.

\begin{tabular}{c|r} 
Coefficient & \multicolumn{1}{|c}{ Value } \\
\hline$c_{1}$ & 8359 \\
$c_{2}$ & 226.2 \\
$c_{3}$ & -827 \\
$c_{4}$ & $8.007 \times 10^{6}$ \\
$c_{5}$ & $4.183 \times 10^{5}$ \\
$c_{6}$ & $-4.873 \times 10^{5}$ \\
$c_{7}$ & $3.424 \times 10^{4}$ \\
$c_{8}$ & 2566 \\
$c_{9}$ & $-1.250 \times 10^{4}$ \\
$c_{10}$ & 1677 \\
$c_{11}$ & $-3.577 \times 10^{4}$ \\
$c_{12}$ & -534.3 \\
$c_{13}$ & 888.8
\end{tabular}

\section{C.2 Coefficients used for non-sinusoidal strain in- put}

The coefficients of the ad-hoc model were found using basin-hopping optimization. The training data consisted of the stress response $\sigma(t)$ to sinusoidal strain

$$
\gamma(t)=\gamma_{0} \sin (2 \pi f t)
$$

with strain amplitudes $\gamma_{0}$ from $1 \%$ to $10 \%$ at a frequency $f=1 \mathrm{~Hz}$. The prediction of the stress response to non-sinusoidal strain input was obtained by integrating the model with the coefficient obtained that way. Non-sinusoidal strain and corresponding strain rate are entered into the model equation resulting in an ODE for the stress response. To increase numerical stability, coefficients left out in [1] were used here, leading to the ODE

$$
\begin{aligned}
\frac{\mathrm{d} \sigma}{\mathrm{d} t}= & c_{1} \gamma+c_{2} \dot{\gamma}+c_{3} \sigma+c_{4} \gamma^{3}+c_{5} \gamma^{2} \dot{\gamma} \\
& +c_{6} \gamma^{2} \sigma+c_{7} \gamma \dot{\gamma}^{2}+c_{8} \gamma \dot{\gamma} \sigma+c_{9} \gamma \sigma^{2} \\
& +c_{10} \dot{\gamma}^{3}+c_{11} \dot{\gamma}^{2} \sigma+c_{12} \dot{\gamma} \sigma^{2}+c_{13} \sigma^{3}
\end{aligned}
$$

The coefficients found for collagen 18CB023001 and used for figure 4.3 are listed in table C.2. 


\section{Acknowledgements}

This thesis and my $\mathrm{PhD}$ as a whole would not have been possible without the help of many others. Although I certainly do not excel at expressing my gratitude in words I would like to try to thank them here.

I was lucky to spend the time of my $\mathrm{PhD}$ in the same group in which I already did my Bachelor thesis - then and now working here meant working in a warm and inspiring atmosphere. I thank our two PIs, Stefan Luther and Ulrich Parlitz, not only for providing me with the opportunity to return to the group for my doctoral studies but also for setting the basis for this special atmosphere. Over the years I had too many great colleagues to name them all - thanks to all of you! With Filippo Cosi I did not only share an office but also the ups and downs of life as a $\mathrm{PhD}$ student. Together with Baltasar Rüchardt, we sabotaged our respective works with numerous coffee breaks and discussions about politics and administration. In my work I am building heavily on Sebastian Stein's PhD thesis - thanks not only for this but for being a great colleague and conference roommate. I am grateful for having had Alexander Schlemmer as a colleague with whom data organization schemes as well as biking trips were planned. I thank my former office mates and further members of our group Thomas Lilienkamp, Sayedeh Hussaini, Raúl Quiñonez, Vineesh Kappadan, Svetlana Husser, Laura Diaz, Sebastian Berg, Tariq Baig, and Daniel Hornung for many discussions about science, programming, and life in general. Daniel also supervised my Bachelor thesis - back then - and introduced me to the world of Emacs. Thanks to all of you who proofread this document, too, and for the many comments and corrections I received from you!

Caroline Hoffrogge, our institute's press officer, was of tremendous support in designing and preparing our presence in the Highlights der Physik exhibition in Dortmund, as were my colleagues Alexander Schlemmer, Jan Lebert, and Jan-Hendrik Plank. Thanks to all of you!

Through our collaboration with the group of Wolfram-Hubertus Zimmermann I received valuable scientific input. Most importantly, it lead to the rheological experiments that Susanne Schlick and I conducted. Thanks for suffering through those with me even when neither gels nor cells proved cooperative. Later on I was assisted in the first experiments with the PBS protocol by Martin Schilling, a Masters student at the Third Physical Institute.

I thank my friends and colleagues Marcel Schröder and Oskar Schnaack for our common coffee brakes at all times that included highly professional analyses of national and international football. Running with Marcel provided an important outlet over the years. 
Ulrich Parlitz supervised my work in the best possible way - while giving me the freedom to follow the ideas I had and do what I deemed right, necessary, or interesting, he was at the same time always available for feedback and guidance. His valuable advice, not only scientifically, contributed greatly to the completion of this project. In the end, "Alles wird gut".

Florian Rehfeldt was not only member of my thesis advisory committee but also my guide into the world of experimental rheology. He was of great help to Susanne and me in devising, conducting, and understanding the experiments. Without his commitment, the experiments would not have produced any meaningful results.

My thesis advisory committee is completed by Stefan Klumpp who over the last three years has been a great source of scientific input. I also thank the further members of my examination board, Karen Alim, Claus Heussinger, and Peter Sollich.

Danken möchte ich an dieser Stelle meinen Eltern, Christiane und Haldun Spreckelsen, die mir durch ihre Unterstützung den Weg des Physikstudiums überhaupt ermöglicht haben. Während all der Zeit wart ihr ein Rückhalt und eine große Hilfe. Mein Großvater, Kay Spreckelsen, führte mich früh an die Physik heran und hatte großen Einfluss auf meinen Werdegang. Danke für Alles, Pali.

Last but not least I want to express my gratitude to the Georg-August University School of Science (GAUSS) for providing a well-organized framework under which this $\mathrm{PhD}$ project was conducted. I am especially grateful for the opportunities to teach in tutorial classes provided by the departments of physics and mathematics during the course of my Bachelor, Master, and PhD studies.

This research was funded by the German Research Foundation (DFG) via the collaborative research center SFB 937, project A18. 